\title{
Nickel-catalyzed Selective Decarbonylation of a-Amino Acid Thioester : Aminomethylation of mercaptans
}

Jing-Ya Zhou, Rui Tian, Yong-Ming Zhu*

†College of Pharmaceutical Sciences, Soochow University, Suzhou 215123, China

*E-mail: zhuyongming@suda.edu.cn

Table of Content

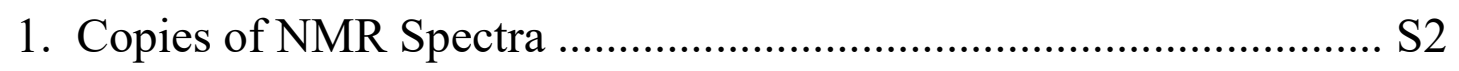

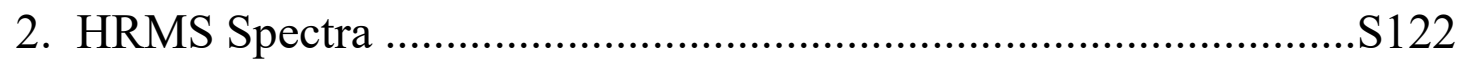

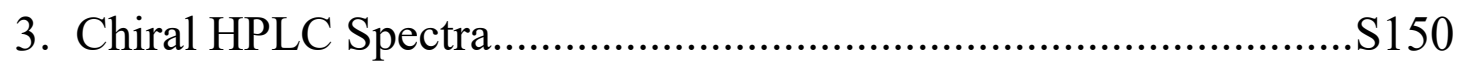


${ }^{1} \mathrm{H}$ NMR (400 MHz, $\mathrm{CDCl}_{3}$ )
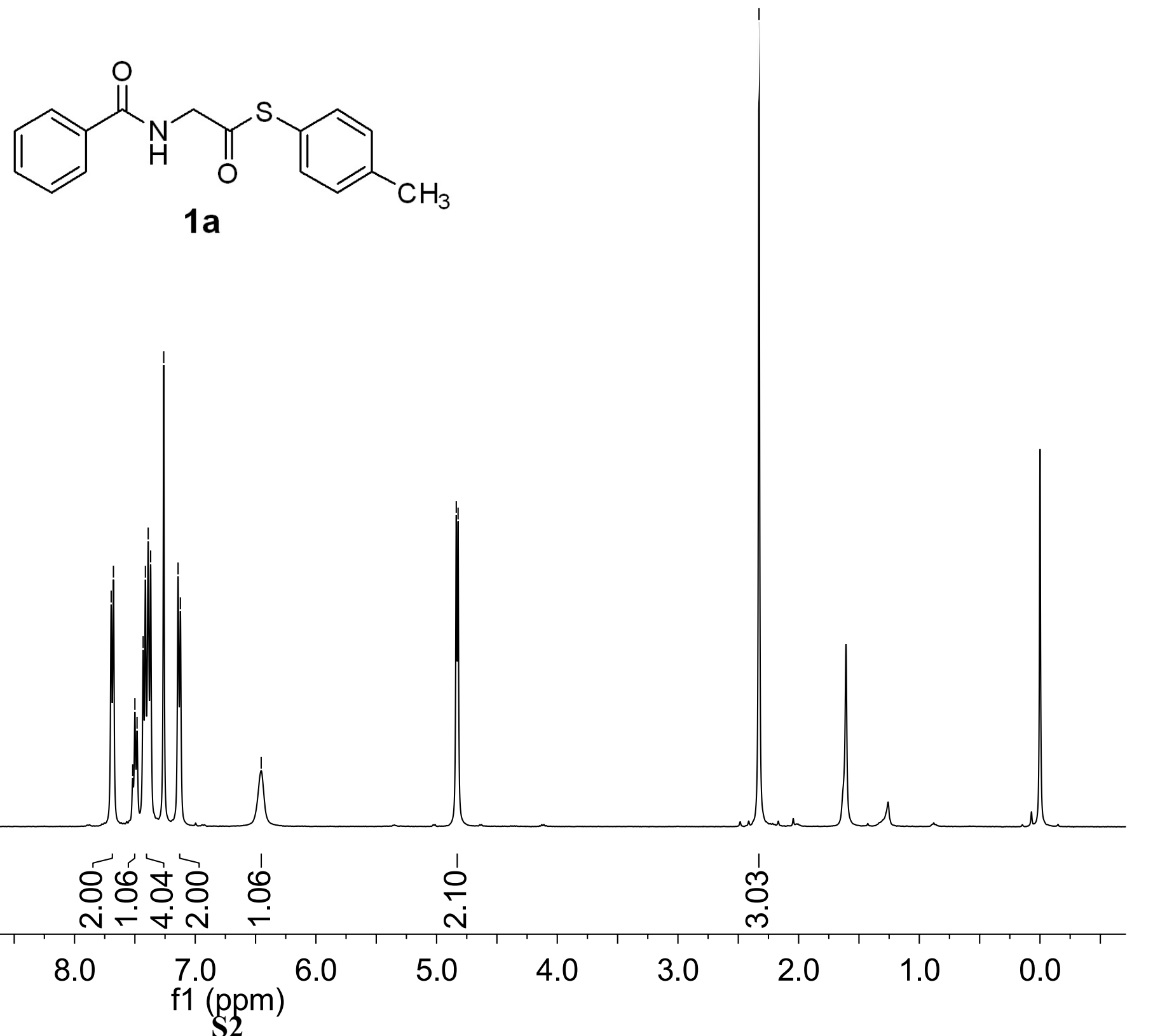
\begin{tabular}{l}
10 \\
0 \\
0 \\
0 \\
\hline \\
\hline
\end{tabular}

${ }^{13} \mathrm{C}\left\{{ }^{1} \mathrm{H}\right\}$ NMR $\left(101 \mathrm{MHz}, \mathrm{CDCl}_{3}\right)$

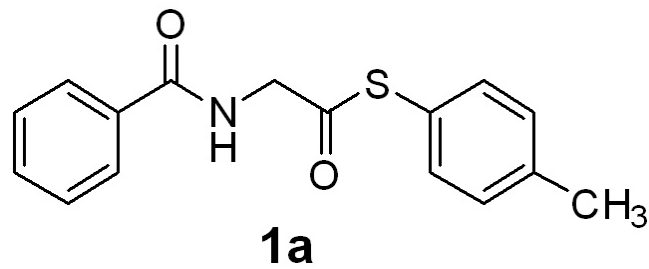

命电

$\stackrel{m}{i}$

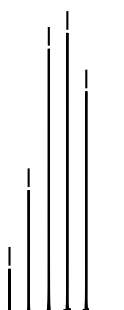

170

150

130

110

90

80

70

60

50

f1 (ppm) 
${ }^{1} \mathrm{H} \mathrm{NMR}\left(400 \mathrm{MHz}, \mathrm{CDCl}_{3}\right)$
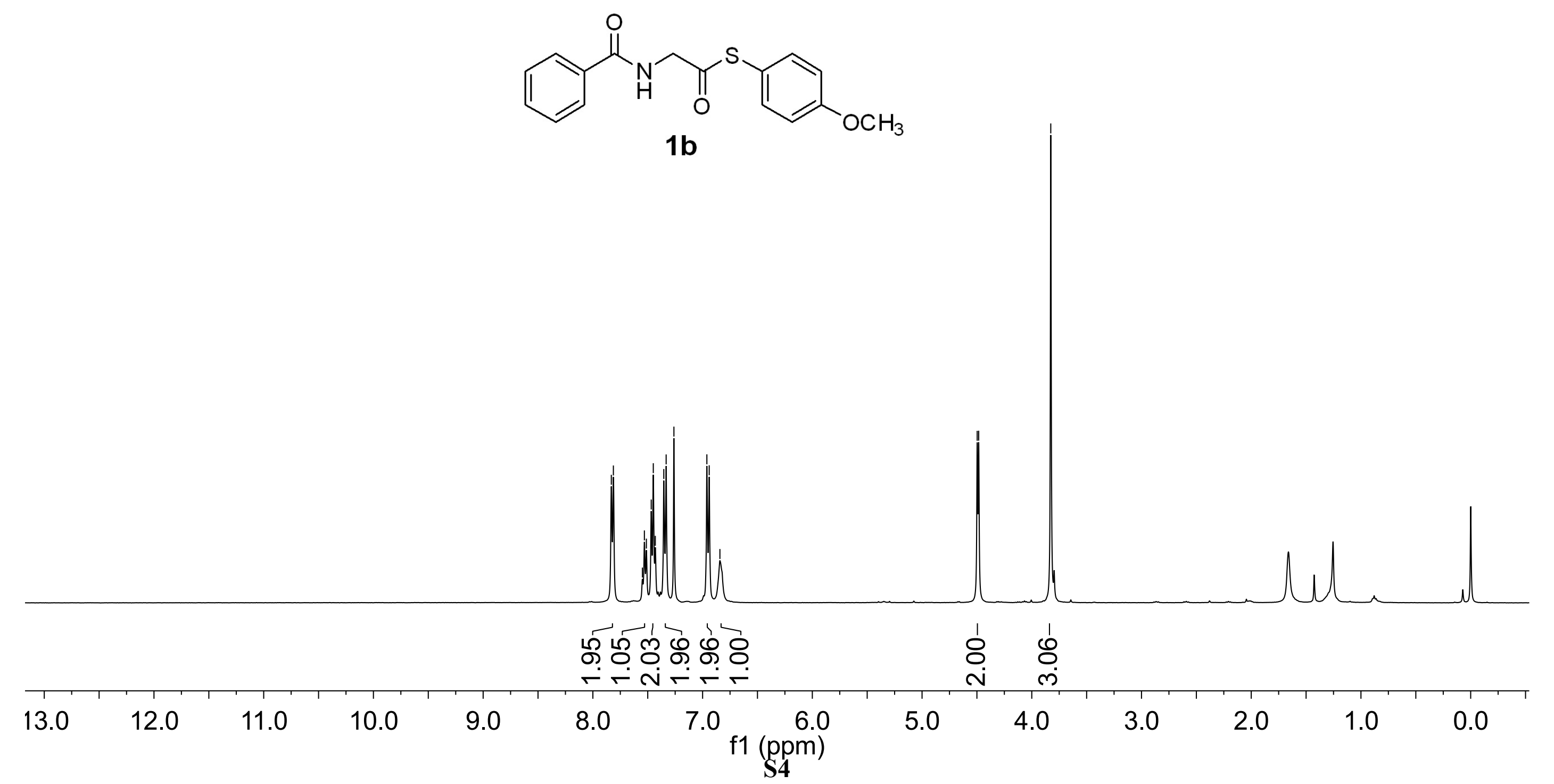
${ }^{13} \mathrm{C}\left\{{ }^{1} \mathrm{H}\right\}$ NMR (101 MHz, $\left.\mathrm{CDCl}_{3}\right)$<smiles>COc1ccc(SC(=O)CNC(=O)c2ccccc2)cc1</smiles>

1b
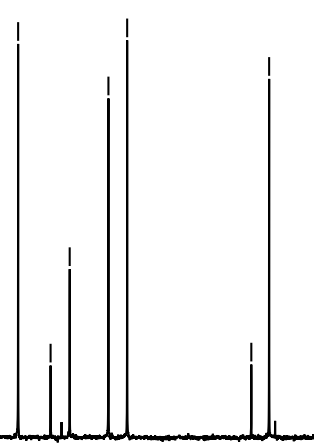

150

130

110

$90 \quad 80$

70

60

50

f1 (ppm) 


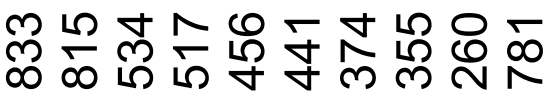

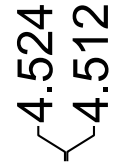

${ }^{1} \mathrm{H}$ NMR $\left(400 \mathrm{MHz}, \mathrm{CDCl}_{3}\right)$
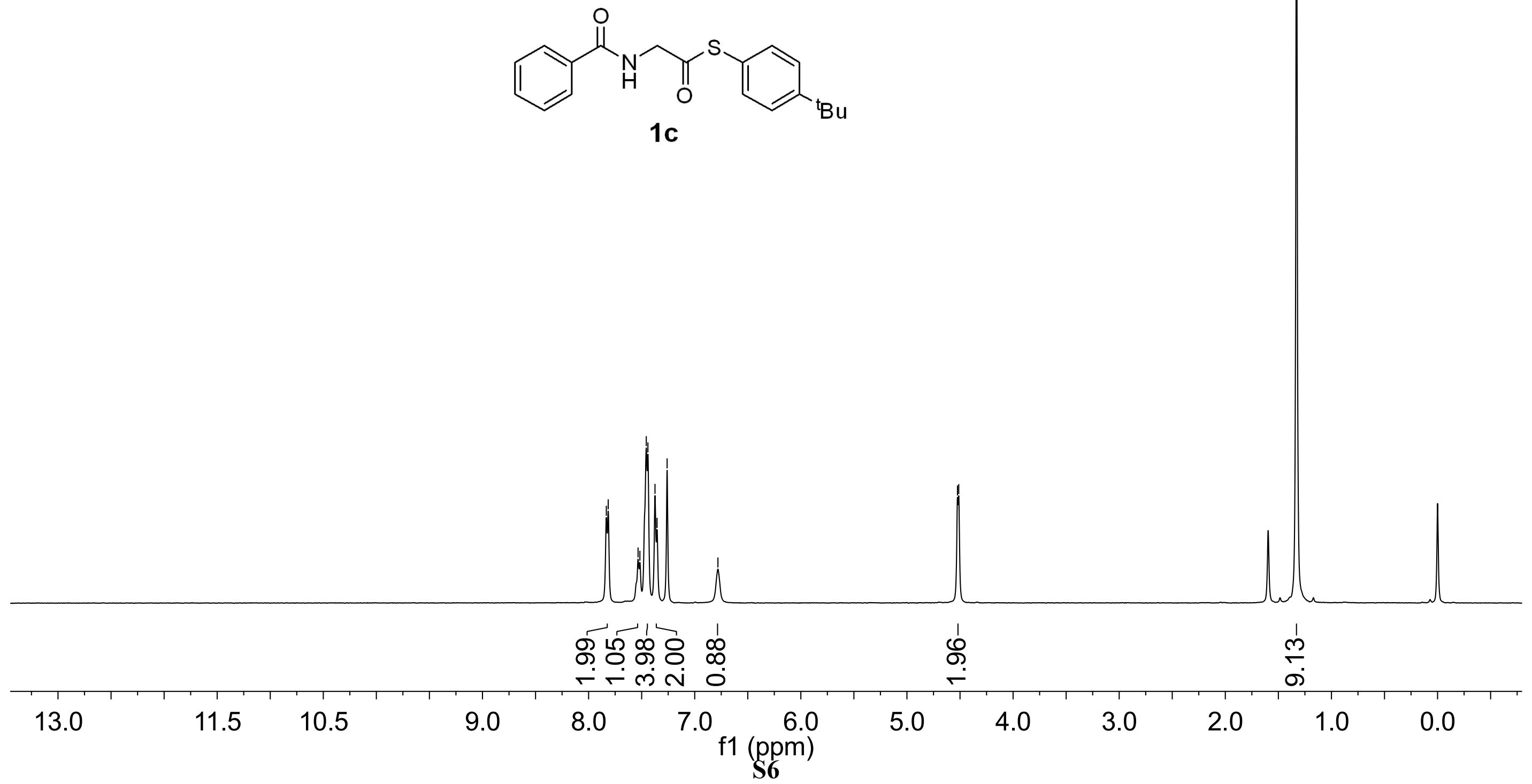


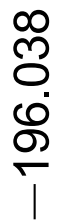

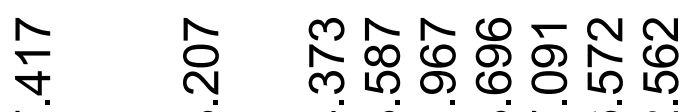

ن

m $8: 0$

ลNo

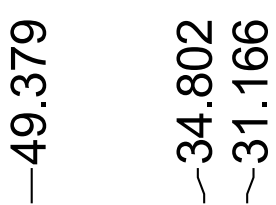

${ }^{13} \mathrm{C}\left\{{ }^{1} \mathrm{H}\right\} \operatorname{NMR}\left(101 \mathrm{MHz}, \mathrm{CDCl}_{3}\right)$
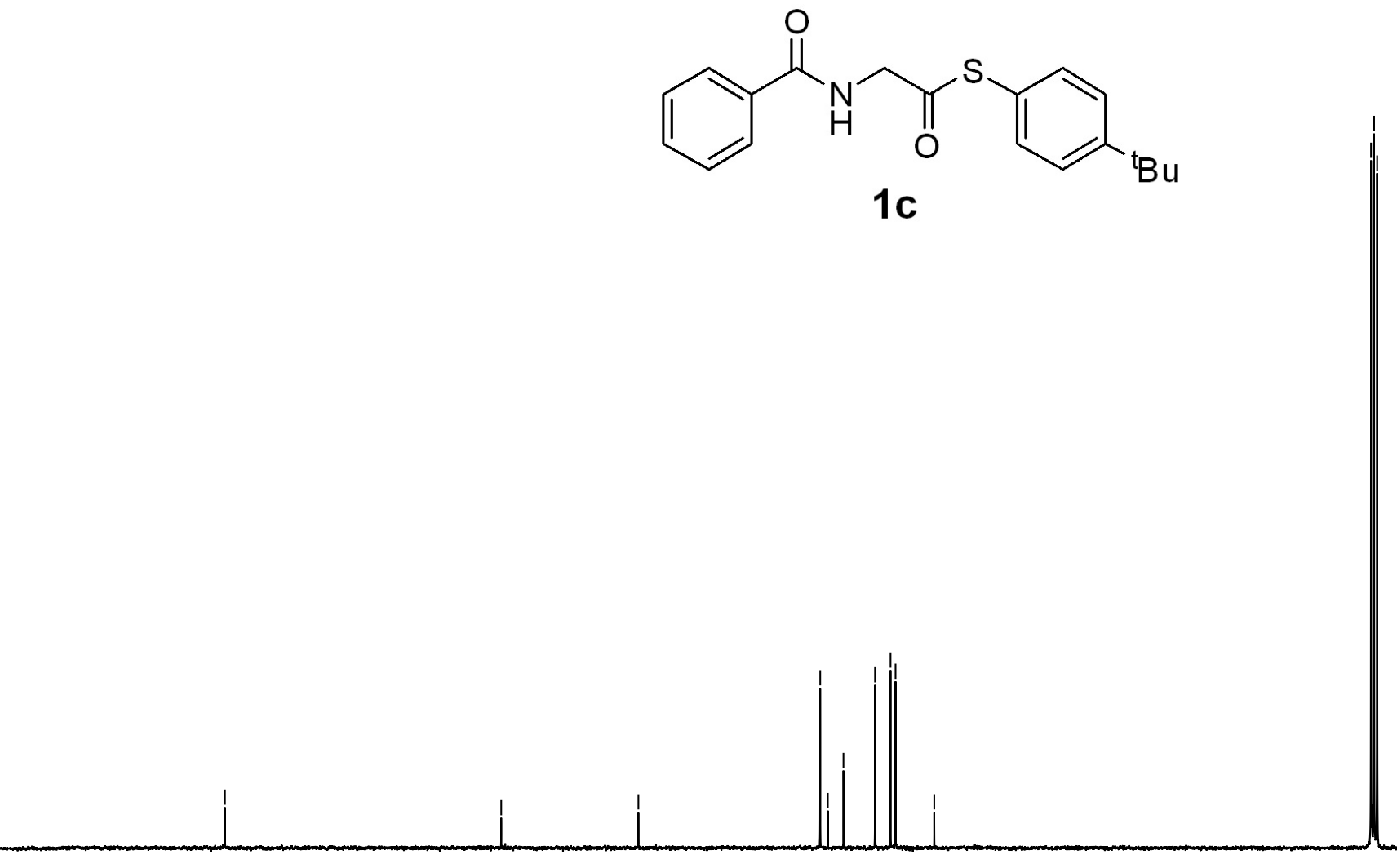

170

150

130

110

90

80

70

$60 \quad 50$

f1 (ppm) 
${ }^{1} \mathrm{H}$ NMR $\left(400 \mathrm{MHz}, \mathrm{CDCl}_{3}\right)$
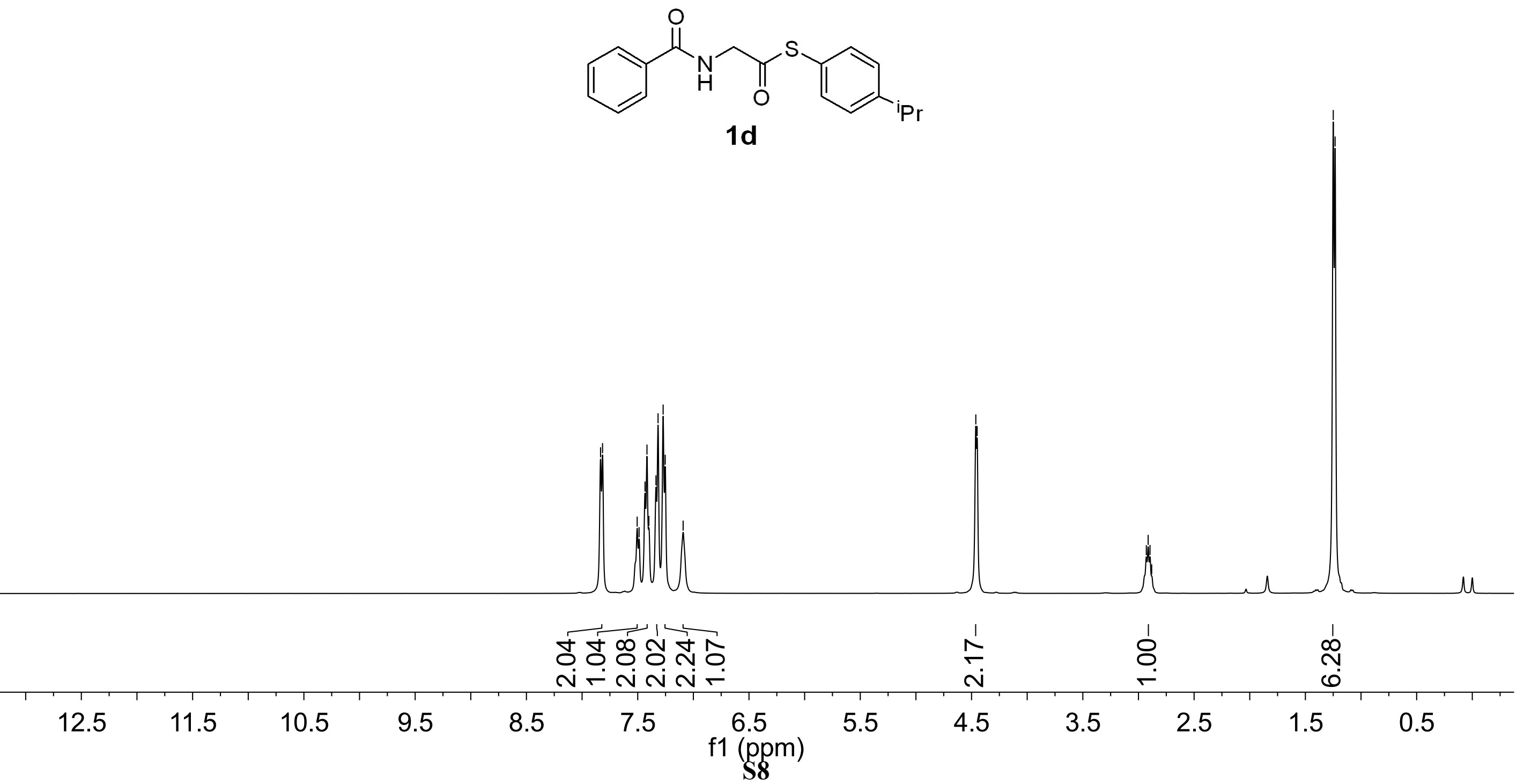
$\mathfrak{N}$
$\stackrel{N}{1}$
$\frac{8}{1}$

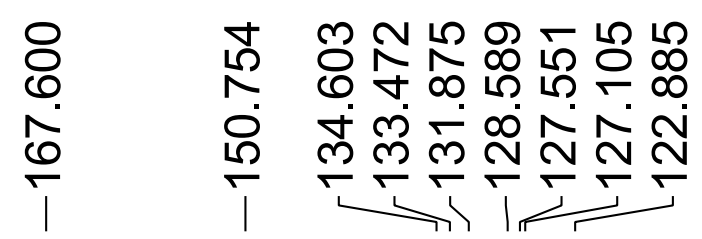

m 8 \&

Nîn

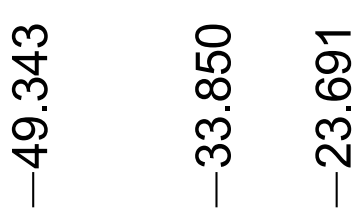

${ }^{13} \mathrm{C}\left\{{ }^{1} \mathrm{H}\right\}$ NMR $\left(101 \mathrm{MHz}, \mathrm{CDCl}_{3}\right)$
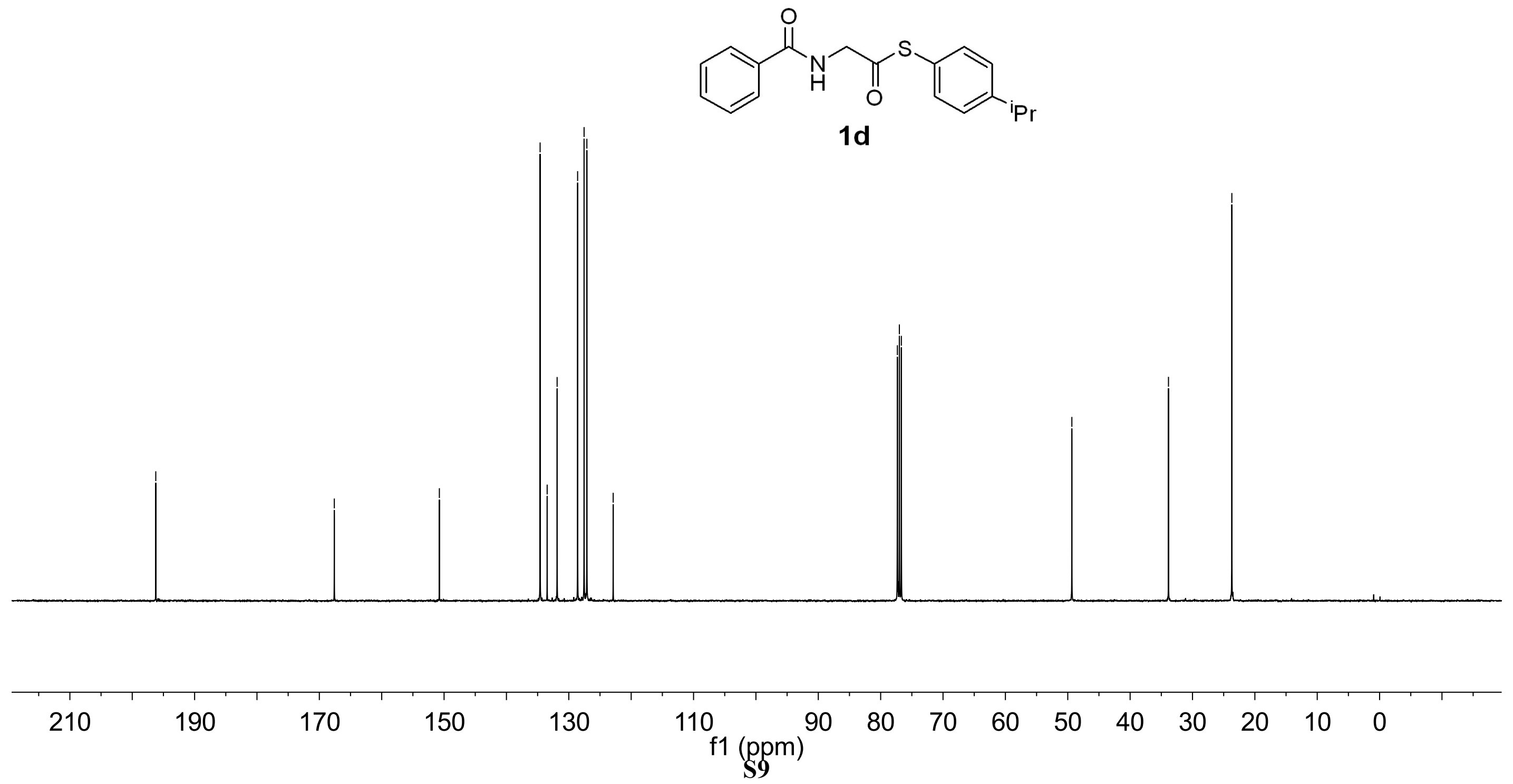
${ }^{1} \mathrm{H}$ NMR $\left(400 \mathrm{MHz}, \mathrm{CDCl}_{3}\right)$
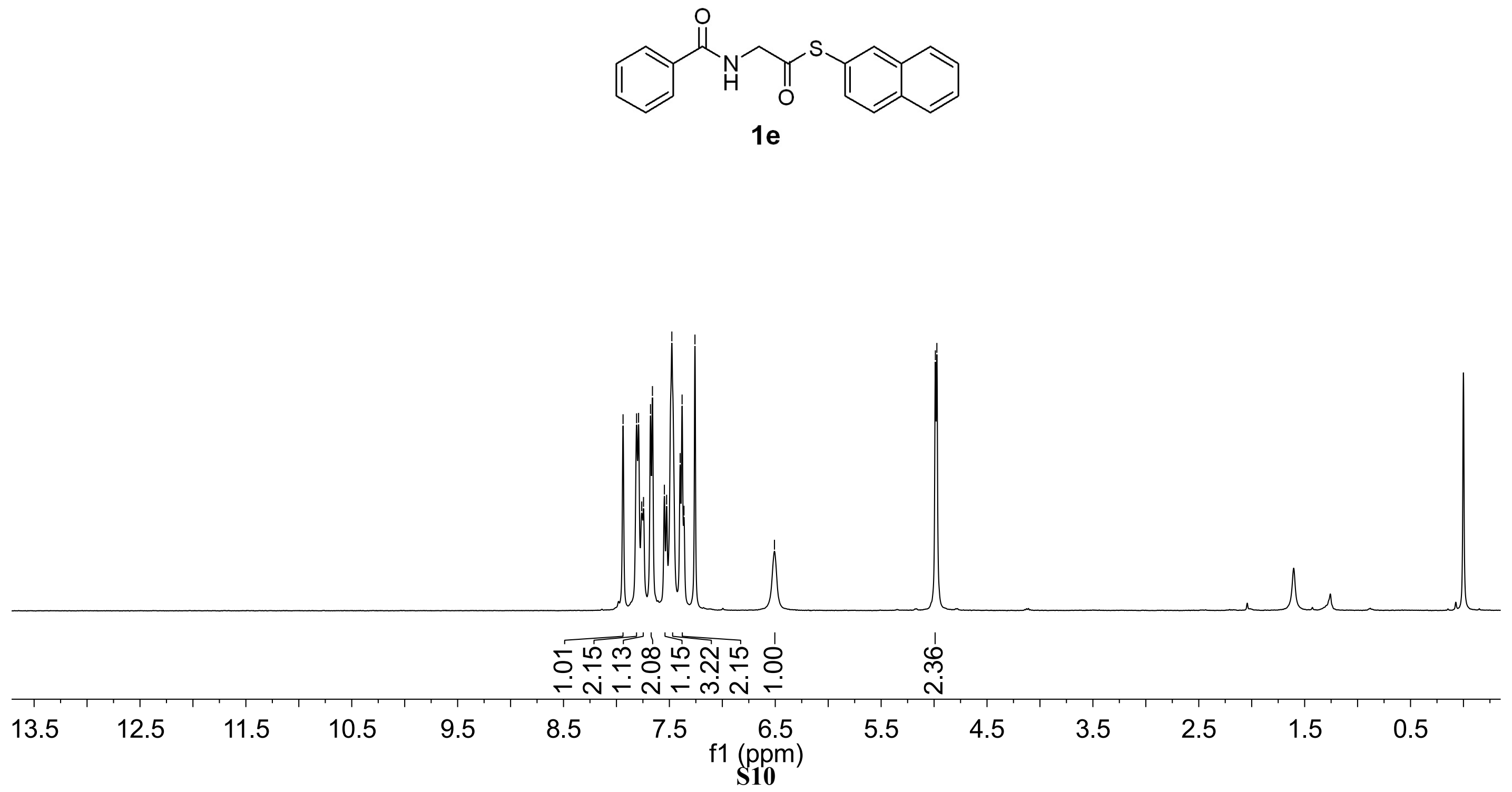
\begin{tabular}{l}
$\infty$ \\
$\infty$ \\
$\infty$ \\
$\stackrel{\circ}{\circ}$ \\
\hline
\end{tabular}

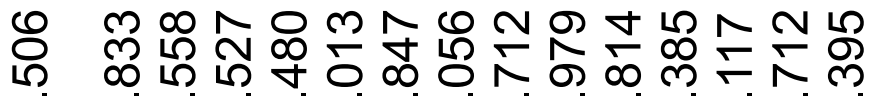

৩o

m 8 \&

人ิ์

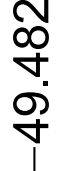

${ }^{13} \mathrm{C}\left\{{ }^{1} \mathrm{H}\right\} \operatorname{NMR}\left(101 \mathrm{MHz}, \mathrm{CDCl}_{3}\right)$
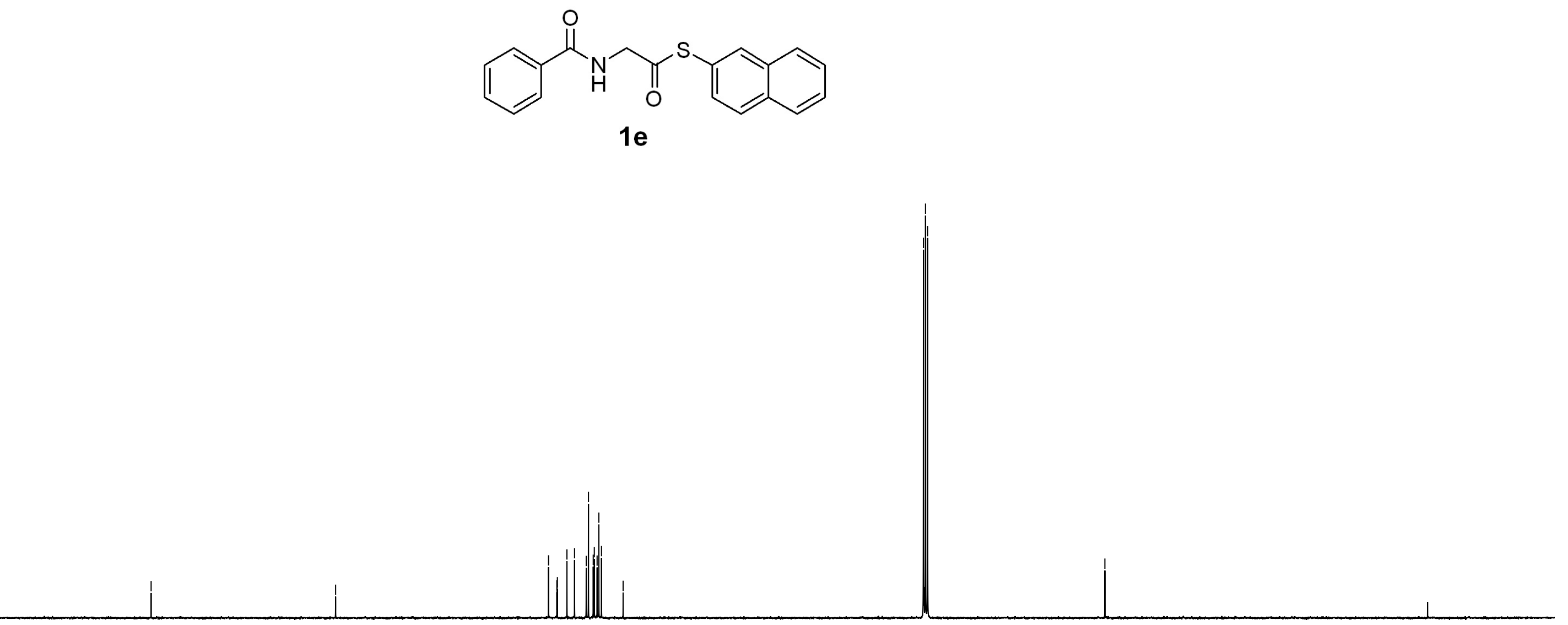

60


${ }^{1} \mathrm{H}$ NMR $\left(400 \mathrm{MHz}, \mathrm{CDCl}_{3}\right)$
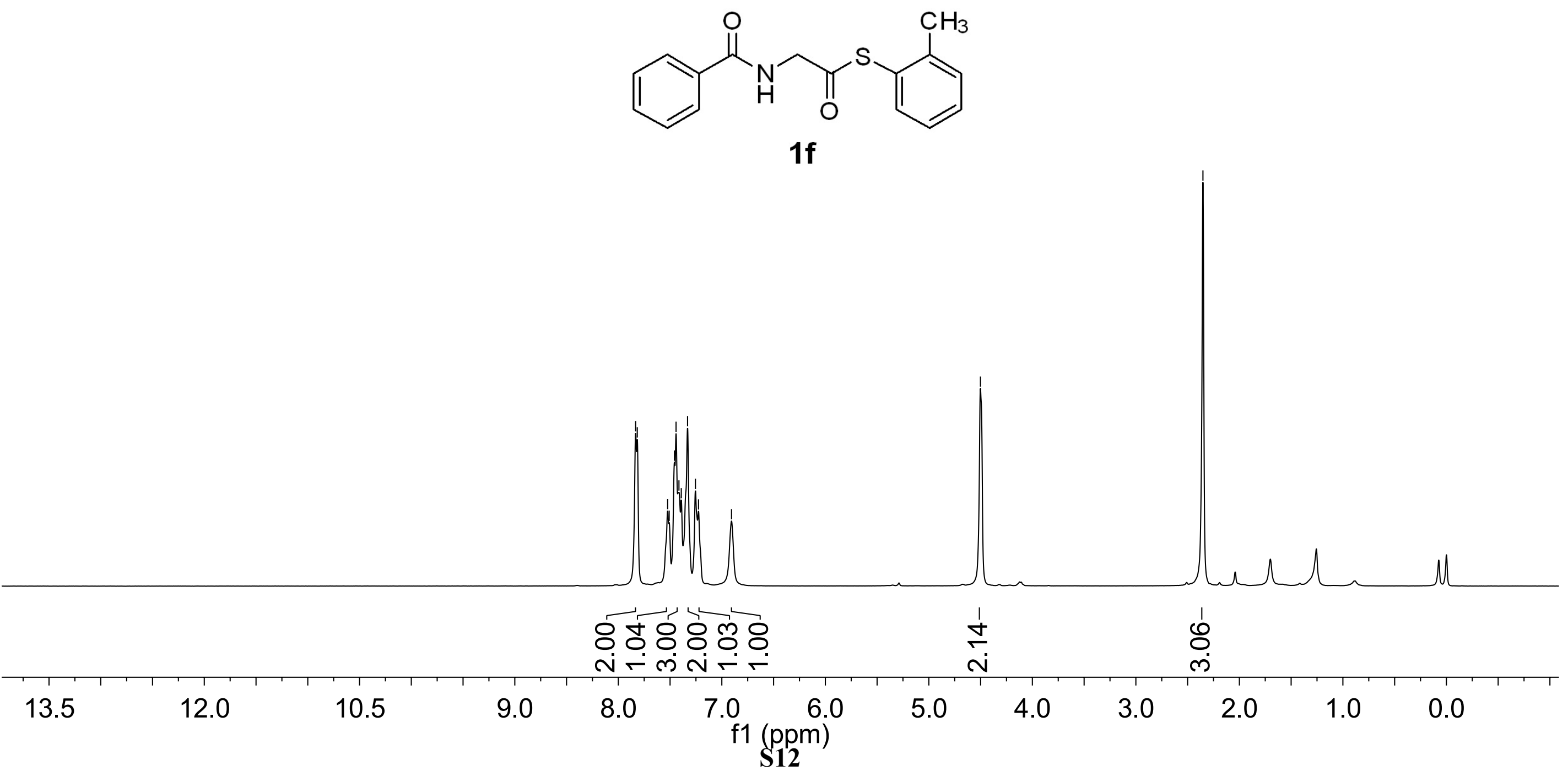


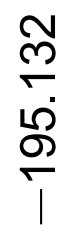

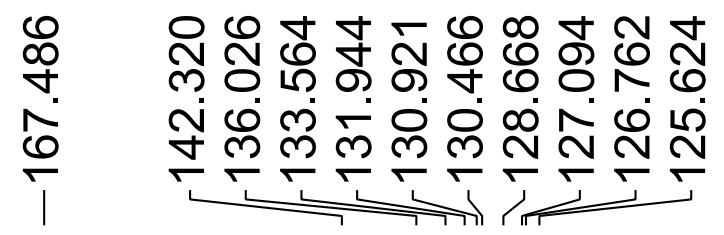

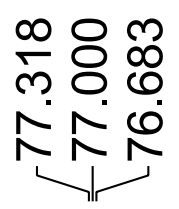

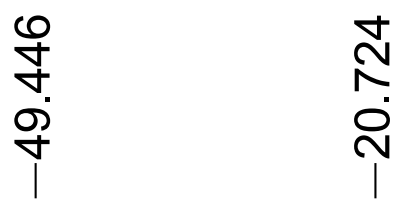

${ }^{13} \mathrm{C}\left\{{ }^{1} \mathrm{H}\right\}$ NMR (101 MHz, $\left.\mathrm{CDCl}_{3}\right)$<smiles>Cc1ccccc1SC(=O)CNC(=O)c1ccccc1</smiles>

$1 f$
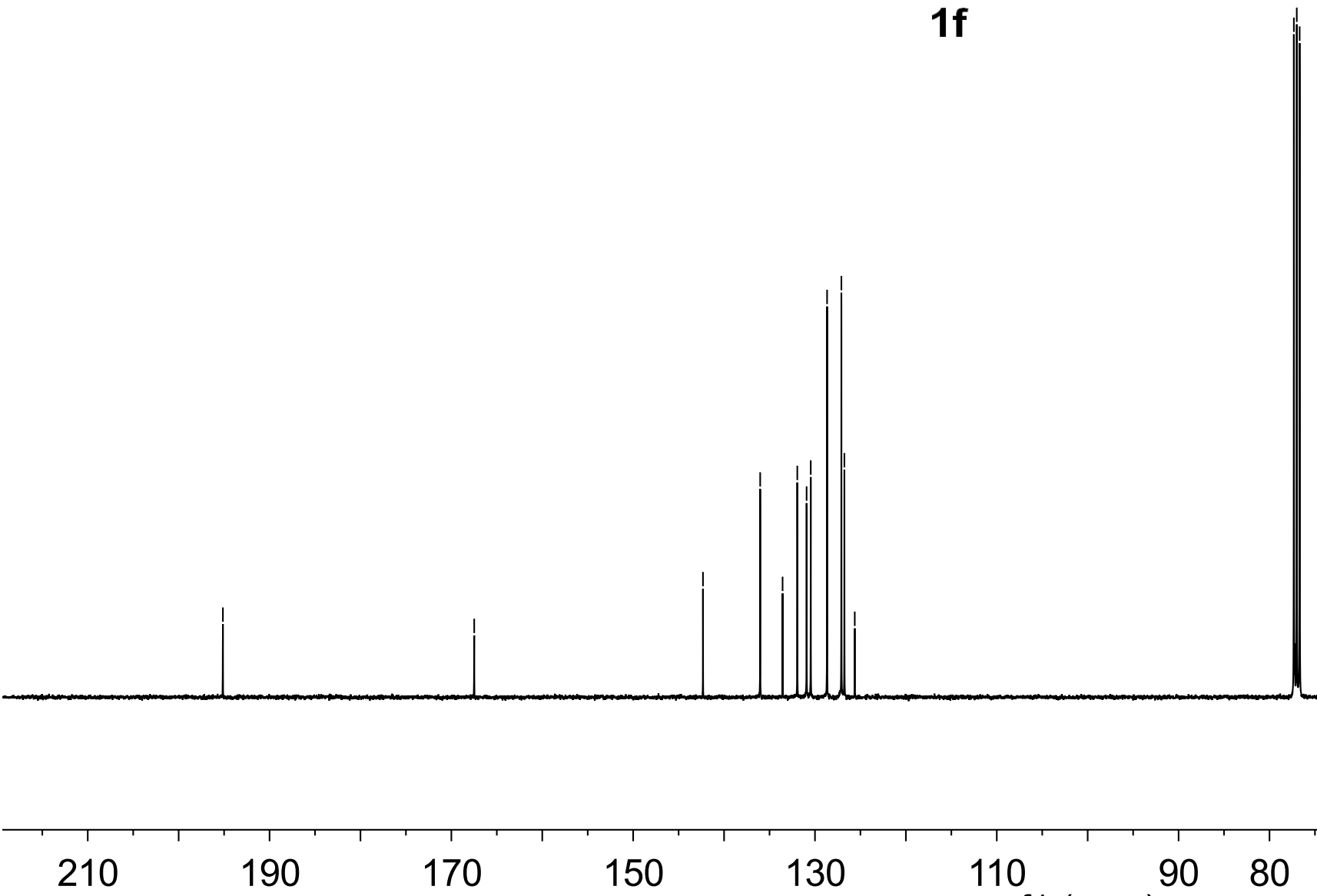

190

170

150

130

110

90

80

70

$60 \quad 50$

f1 (ppm) 
${ }^{1} \mathrm{H}$ NMR (400 MHz, $\left.\mathrm{CDCl}_{3}\right)$
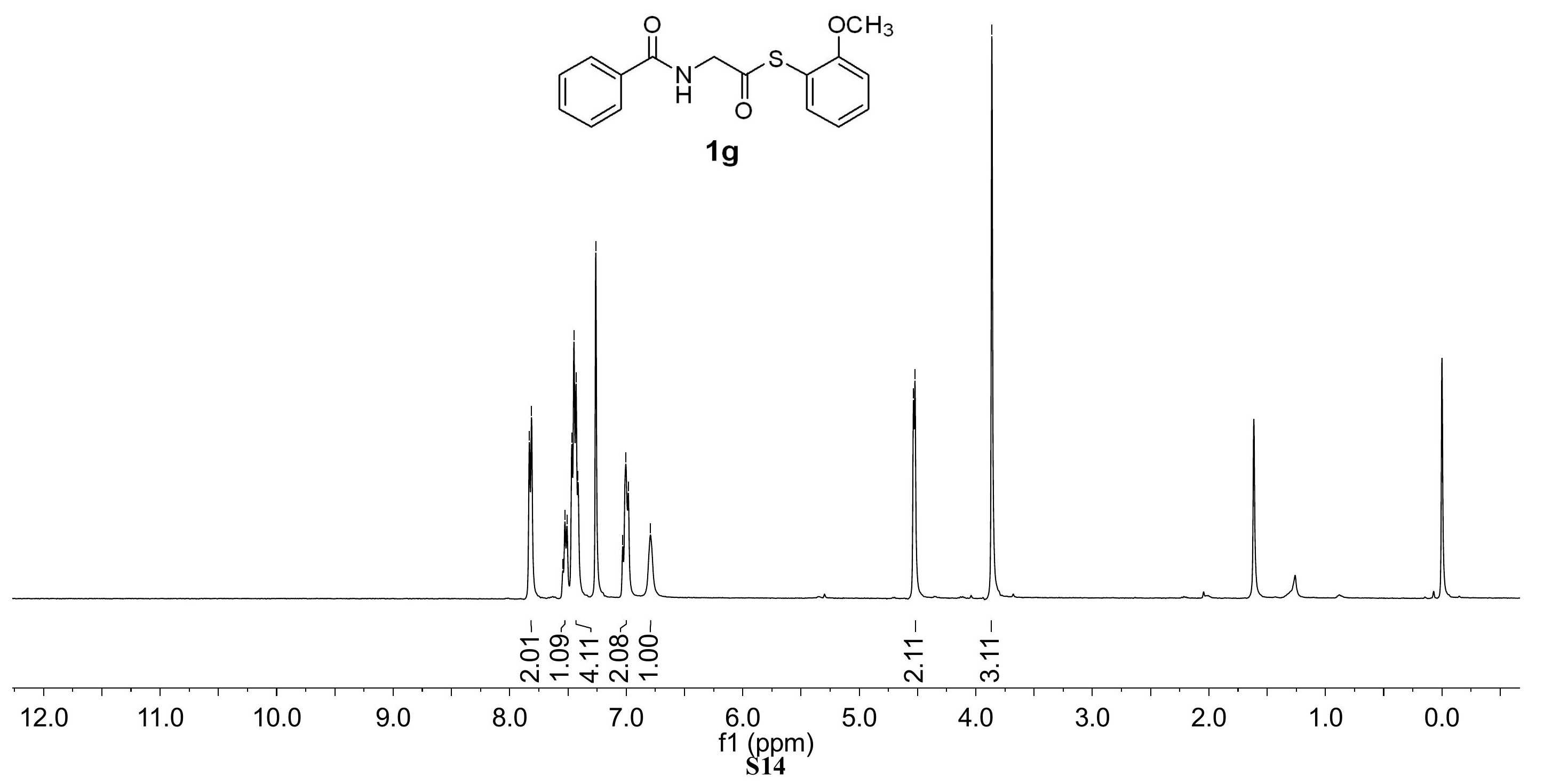
$\infty$
8
0
$\frac{0}{1}$

${ }^{13} \mathrm{C}\left\{{ }^{1} \mathrm{H}\right\} \mathrm{NMR}\left(101 \mathrm{MHz}, \mathrm{CDCl}_{3}\right)$<smiles>COc1ccccc1SC(=O)CNC(=O)c1ccccc1</smiles> 
${ }^{1} \mathrm{H}$ NMR $\left(400 \mathrm{MHz}, \mathrm{CDCl}_{3}\right)$

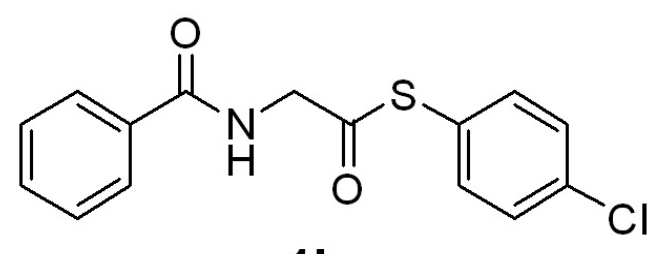

$1 \mathrm{~h}$

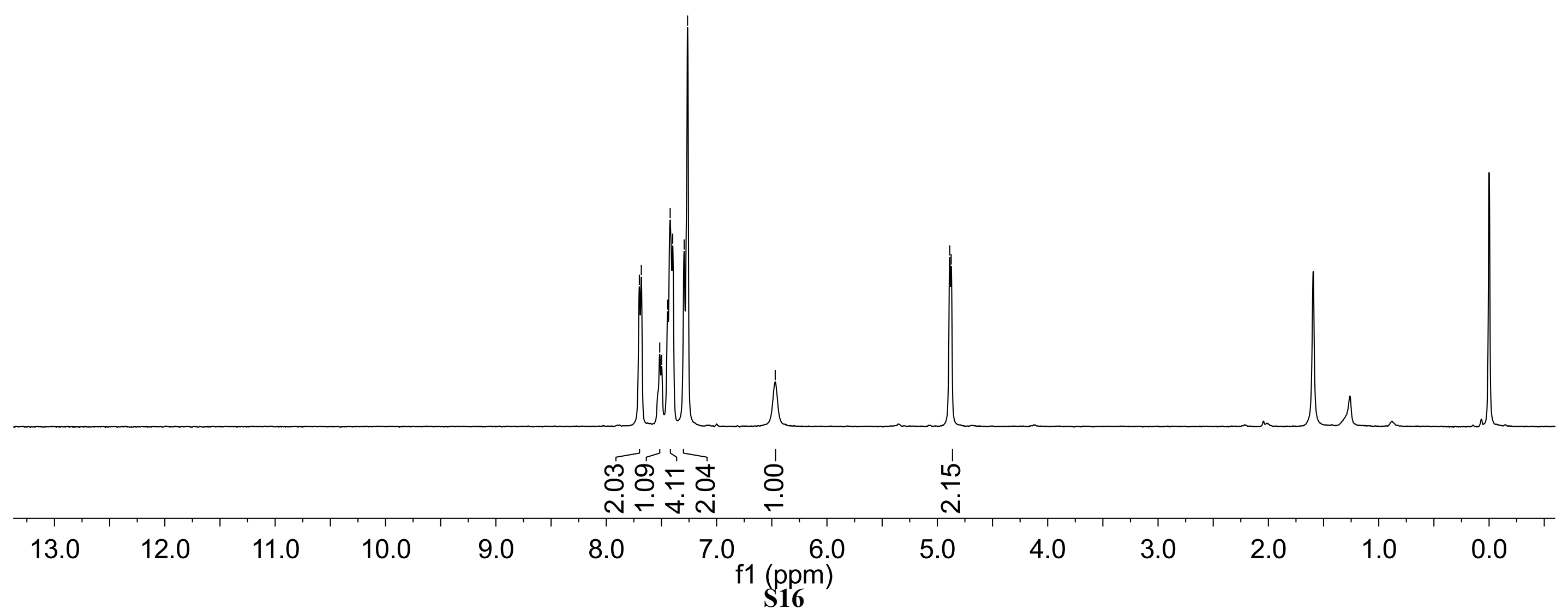




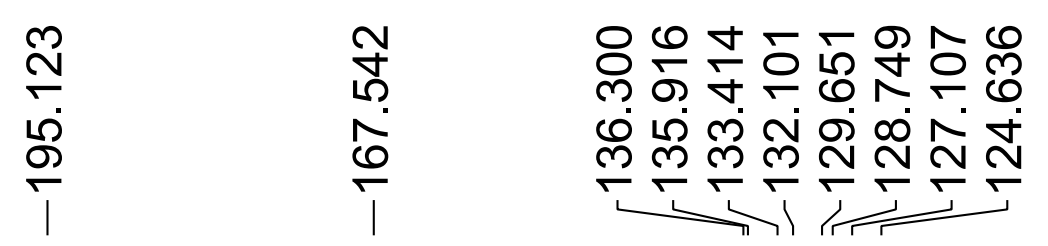

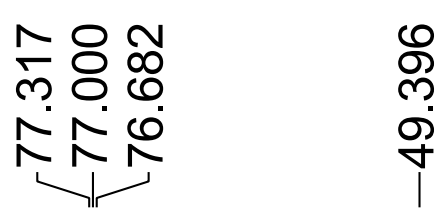

${ }^{13} \mathrm{C}\left\{{ }^{1} \mathrm{H}\right\} \mathrm{NMR}\left(101 \mathrm{MHz}, \mathrm{CDCl}_{3}\right)$<smiles>O=C(CNC(=O)c1ccccc1)Sc1ccc(Cl)cc1</smiles>

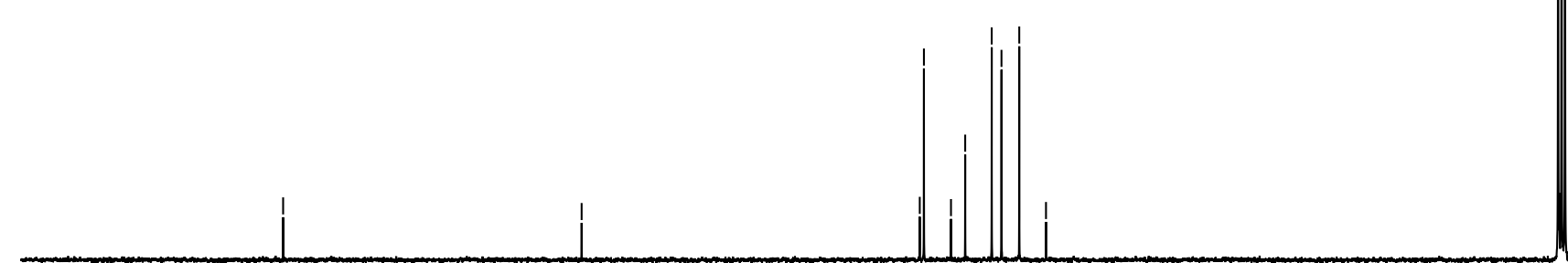

\begin{tabular}{llllllllllllllllll}
\hline 210 & 190 & 170 & 150 & 130 & $\begin{array}{c}110 \\
\mathrm{f1}(\mathrm{ppm})\end{array}$ & 90 & 80 & 70 & 60 & 50 & 40 & 30 & 20 & 10 & 0 &
\end{tabular}


${ }^{1} \mathrm{H}$ NMR $\left(400 \mathrm{MHz}, \mathrm{CDCl}_{3}\right)$
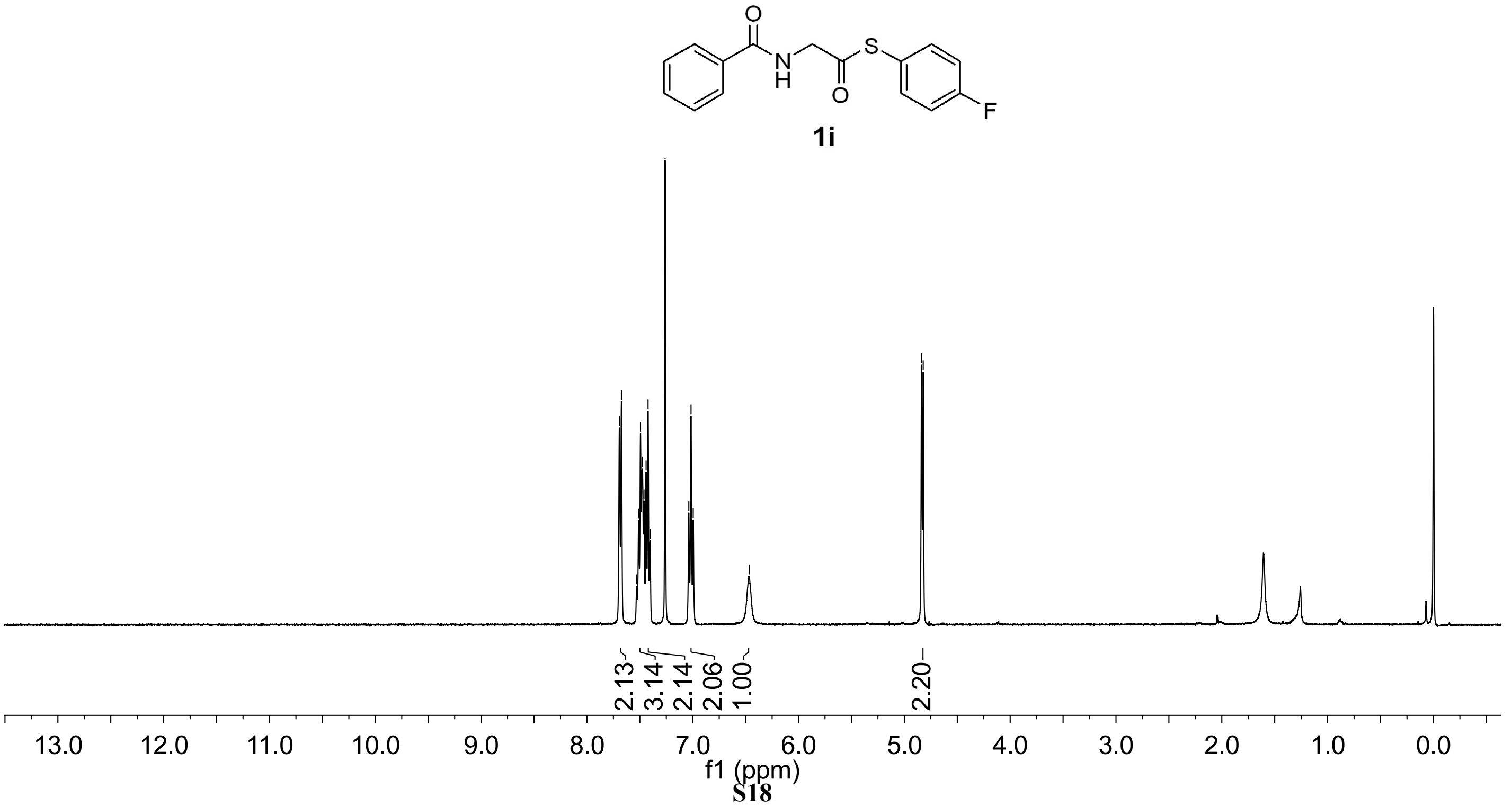


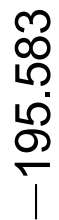

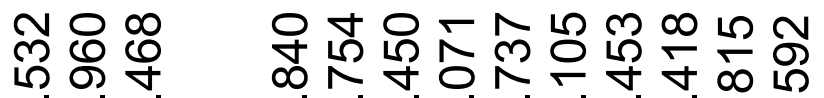
ำ m̋ 음 F

$\infty$

ले 0

NヘN

r

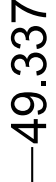

${ }^{13} \mathrm{C}\left\{{ }^{1} \mathrm{H}\right\} \operatorname{NMR}\left(101 \mathrm{MHz}, \mathrm{CDCl}_{3}\right)$
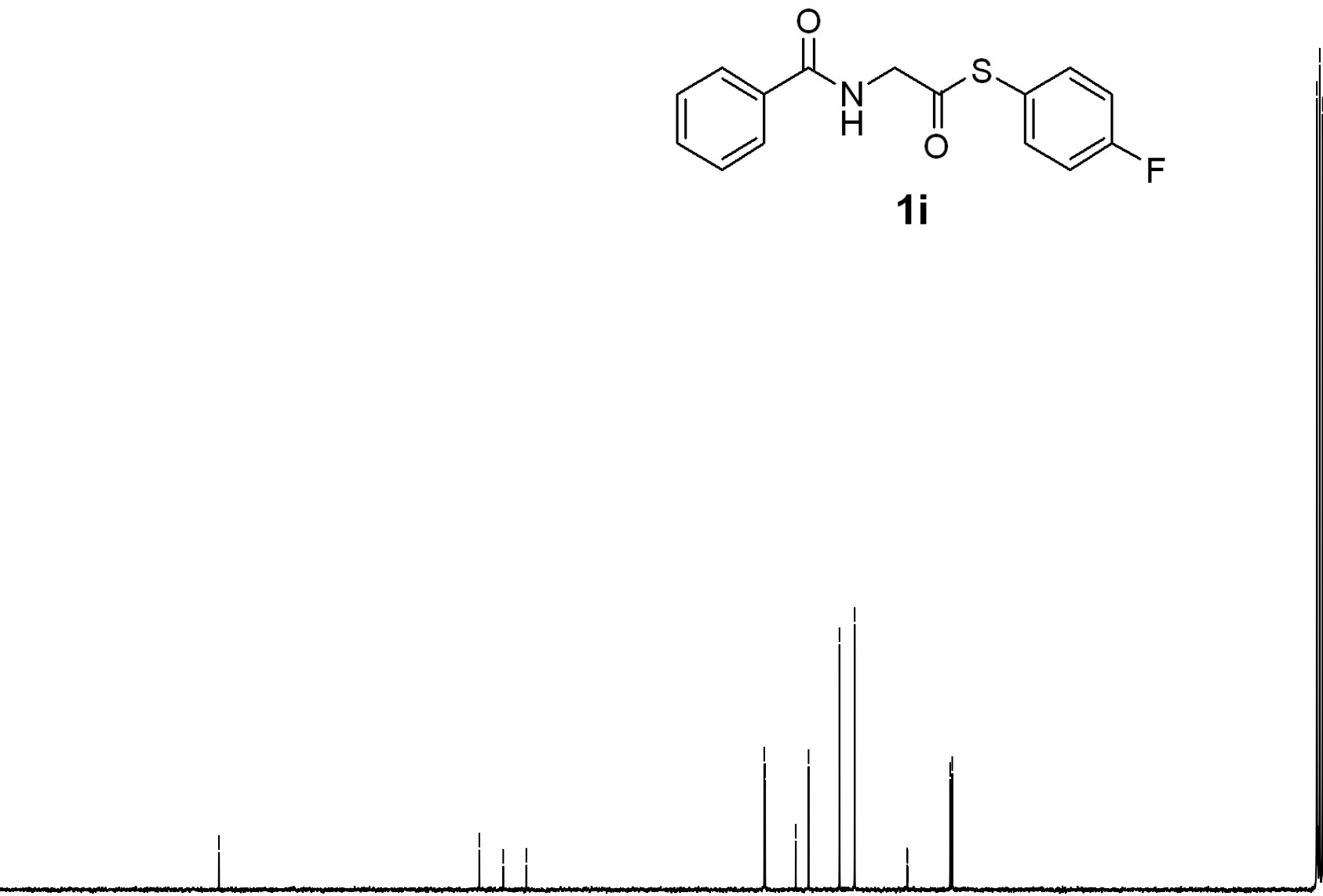

170

150

130

110

90

80

70

f1 (ppm)

$\begin{array}{lllllll}50 & 40 & 30 & 20 & 10 & 0\end{array}$


${ }^{1} \mathrm{H} \mathrm{NMR}\left(400 \mathrm{MHz}, \mathrm{CDCl}_{3}\right)$
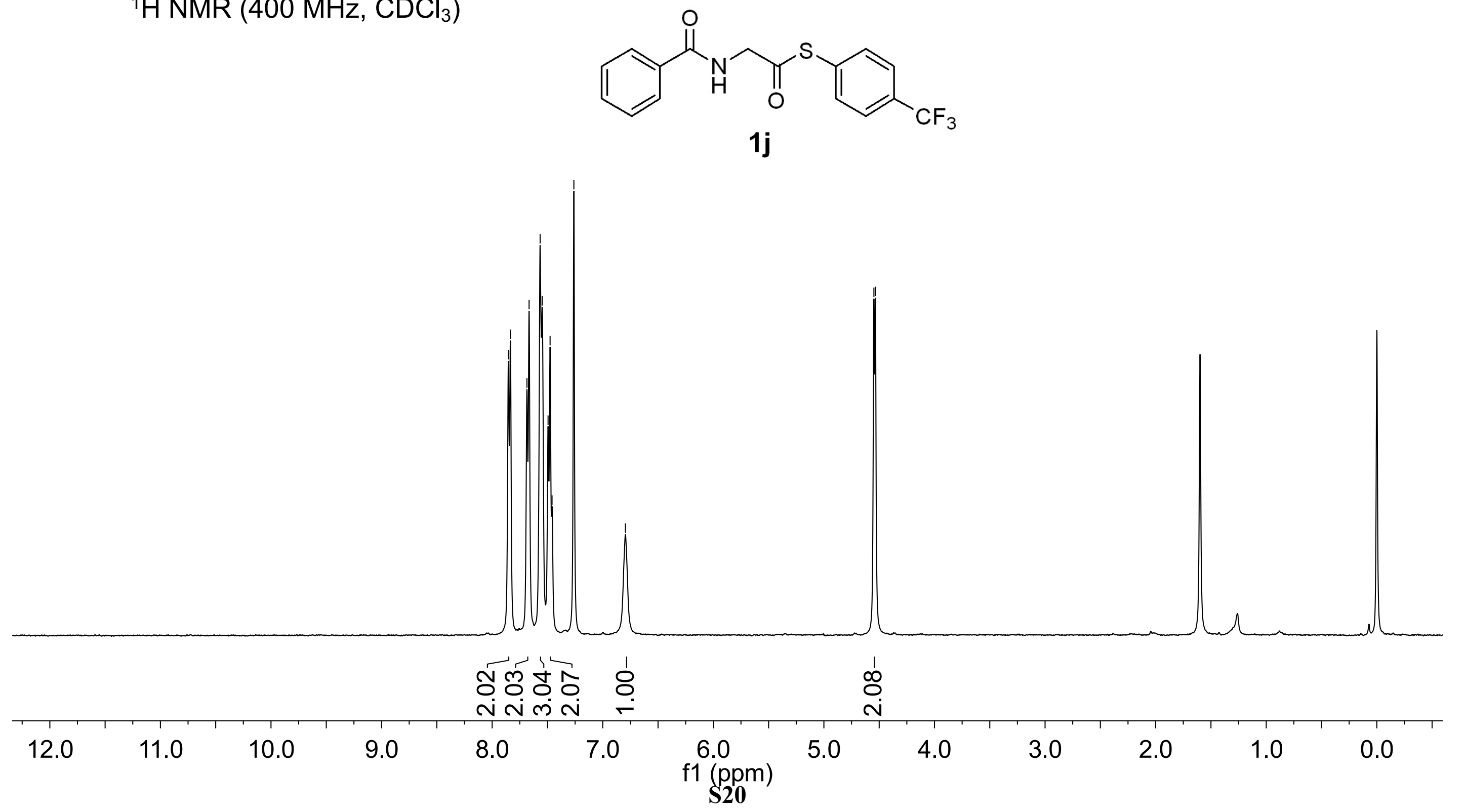
${ }^{13} \mathrm{C}\left\{{ }^{1} \mathrm{H}\right\}$ NMR $\left(101 \mathrm{MHz}, \mathrm{CDCl}_{3}\right)$
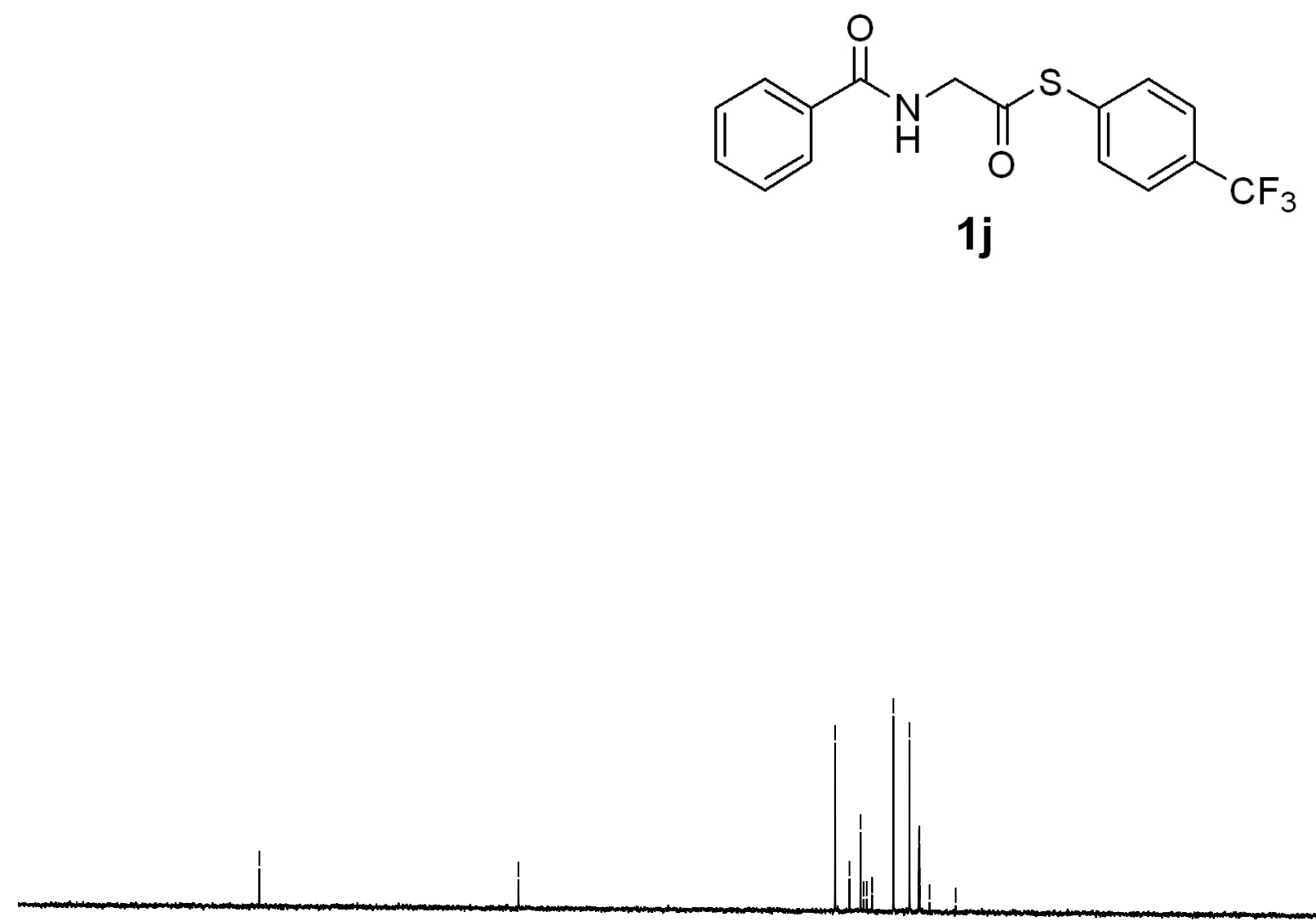

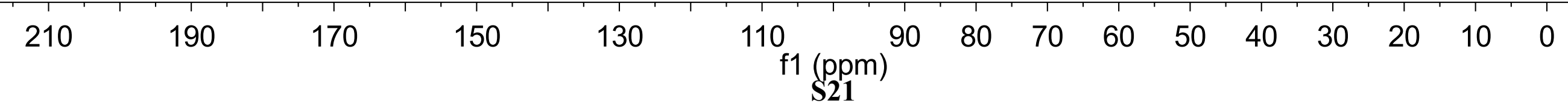


${ }^{1} \mathrm{H}$ NMR $\left(400 \mathrm{MHz}, \mathrm{CDCl}_{3}\right)$<smiles>O=C(CNC(=O)c1ccccc1)Sc1ccc(F)c(F)c1</smiles>

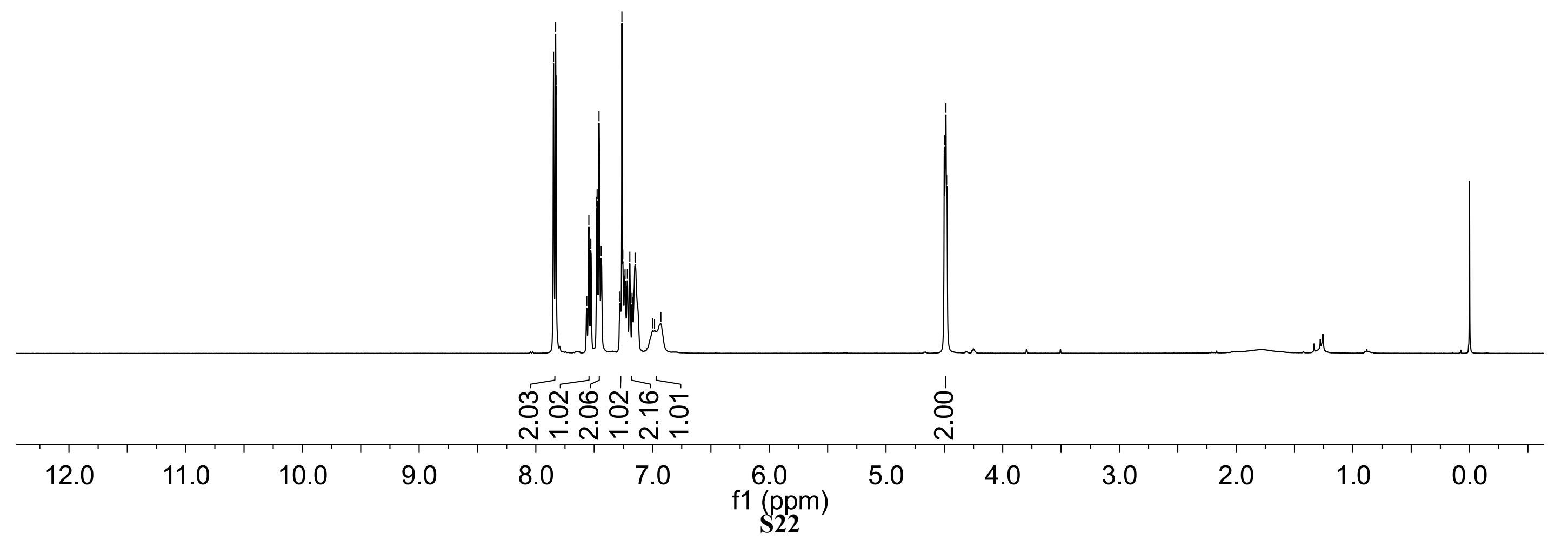


${ }^{13} \mathrm{C}\left\{{ }^{1} \mathrm{H}\right\}$ NMR $\left(101 \mathrm{MHz}, \mathrm{CDCl}_{3}\right)$
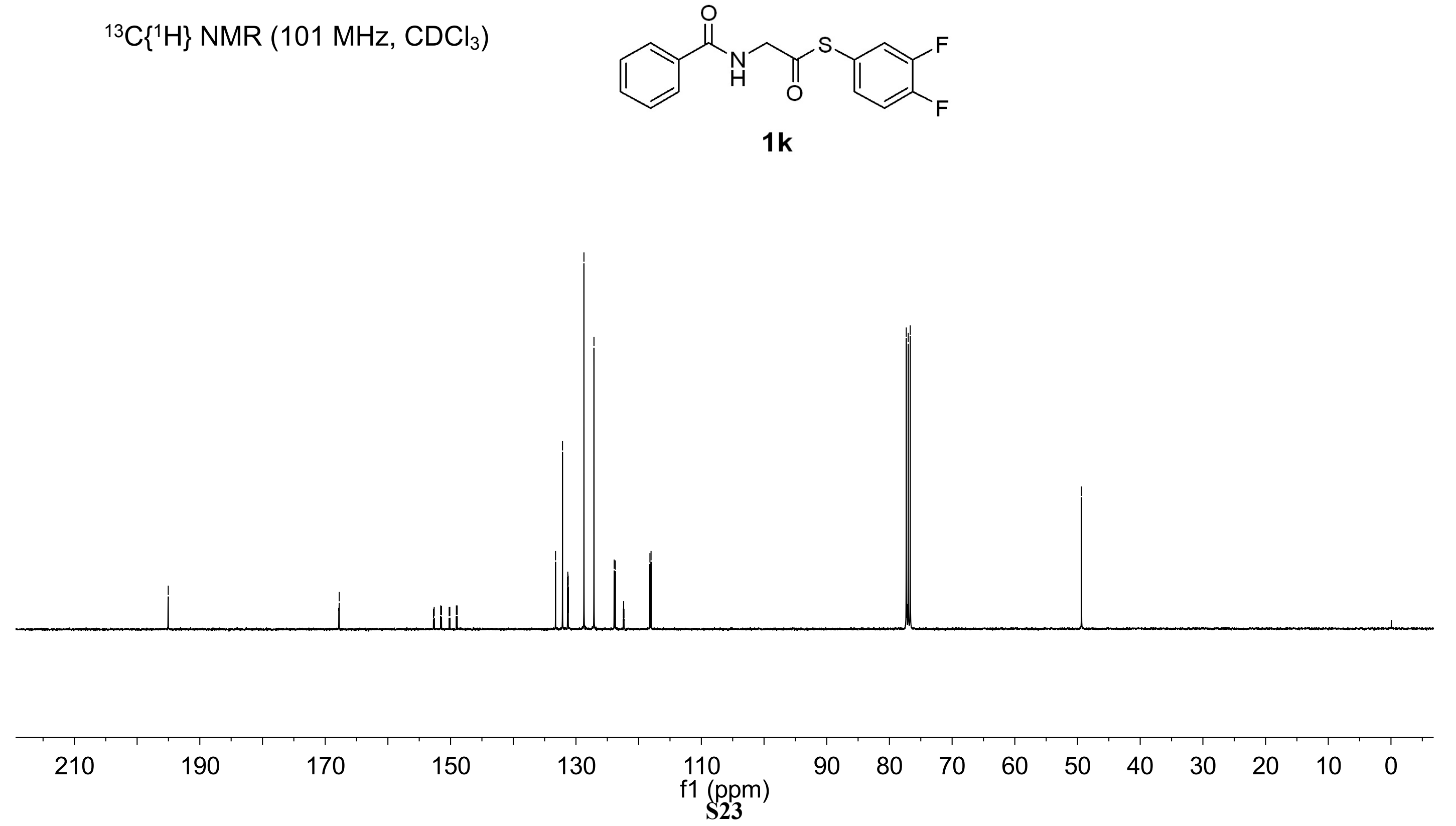
${ }^{1} \mathrm{H}$ NMR $\left(400 \mathrm{MHz}, \mathrm{CDCl}_{3}\right)$
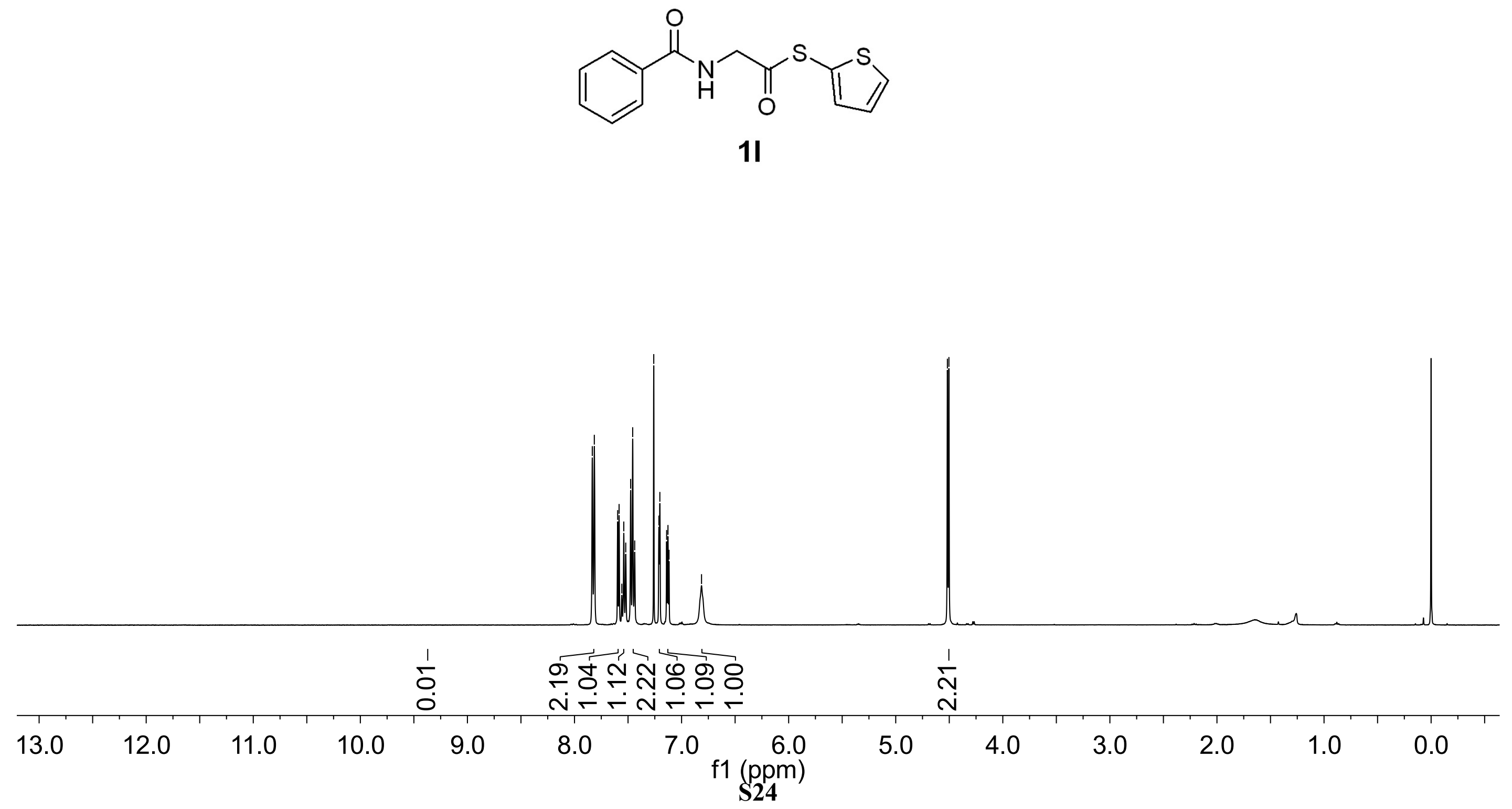
${ }^{13} \mathrm{C}\left\{{ }^{1} \mathrm{H}\right\} \operatorname{NMR}\left(101 \mathrm{MHz}, \mathrm{CDCl}_{3}\right)$<smiles>O=C(CNC(=O)c1ccccc1)Sc1cccs1</smiles>

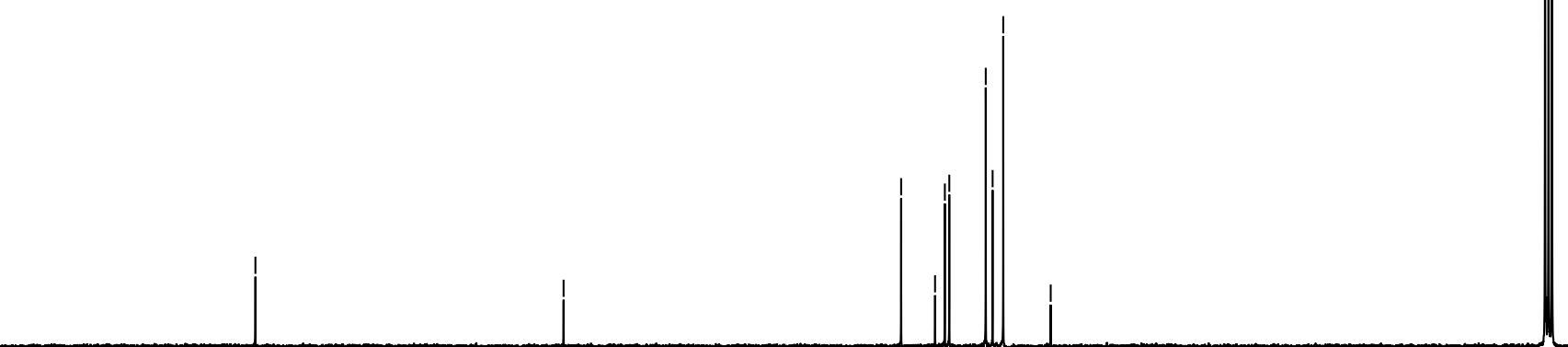


${ }^{1} \mathrm{H}$ NMR $\left(400 \mathrm{MHz}, \mathrm{CDCl}_{3}\right)$<smiles>Cc1occc1SC(=O)CNC(=O)c1ccccc1</smiles>

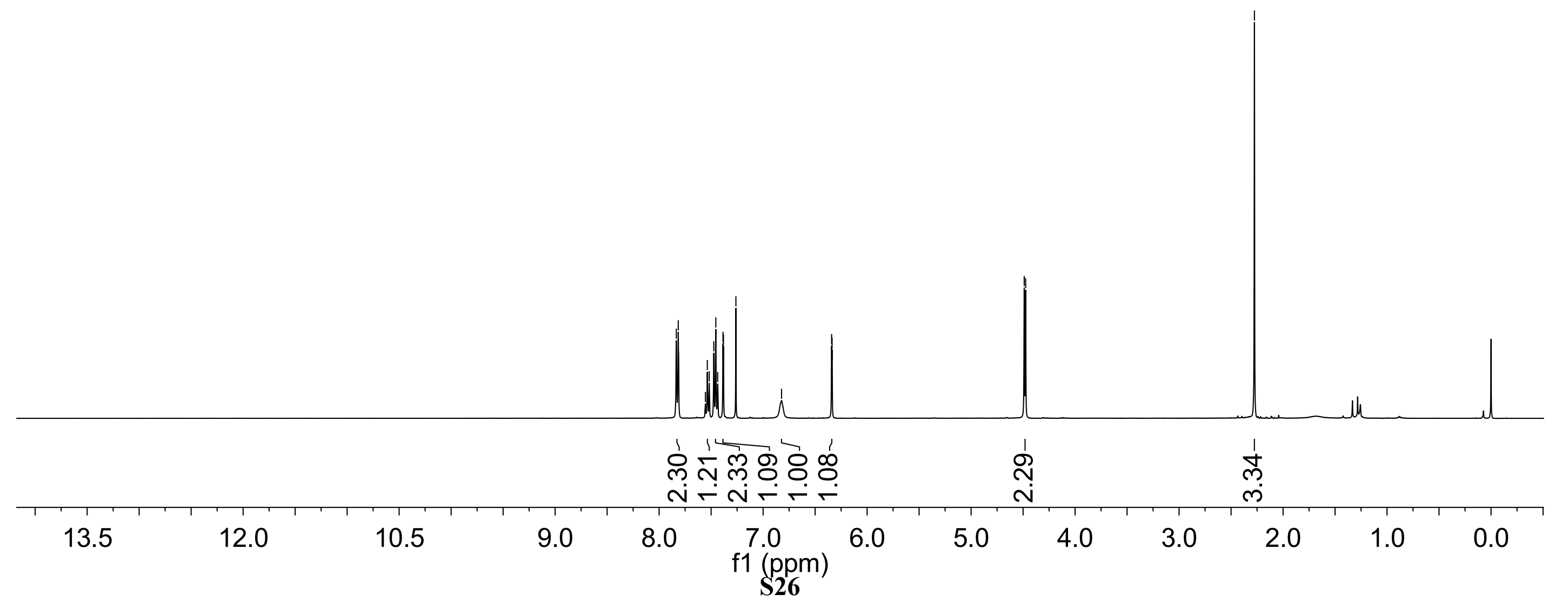




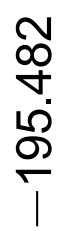

${ }^{13} \mathrm{C}\left\{{ }^{1} \mathrm{H}\right\} \operatorname{NMR}\left(101 \mathrm{MHz}, \mathrm{CDCl}_{3}\right)$<smiles>Cc1occc1SC(=O)CNC(=O)c1ccccc1</smiles> 
${ }^{1} \mathrm{H}$ NMR $\left(400 \mathrm{MHz}, \mathrm{CDCl}_{3}\right)$<smiles>CC1(C)CC1SC(=O)CNC(=O)c1ccccc1</smiles>

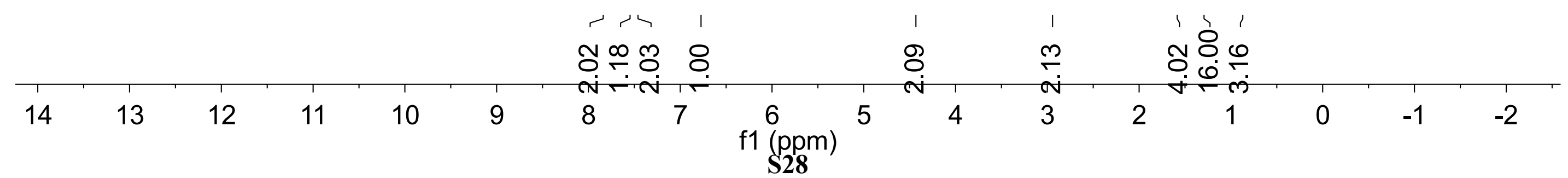


${ }^{13} \mathrm{C}\left\{{ }^{1} \mathrm{H}\right\} \operatorname{NMR}\left(101 \mathrm{MHz}, \mathrm{CDCl}_{3}\right)$<smiles>CC1(C)CC1SC(=O)CNC(=O)c1ccccc1</smiles>

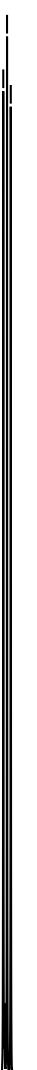


${ }^{1} \mathrm{H}$ NMR $\left(400 \mathrm{MHz}, \mathrm{CDCl}_{3}\right)$
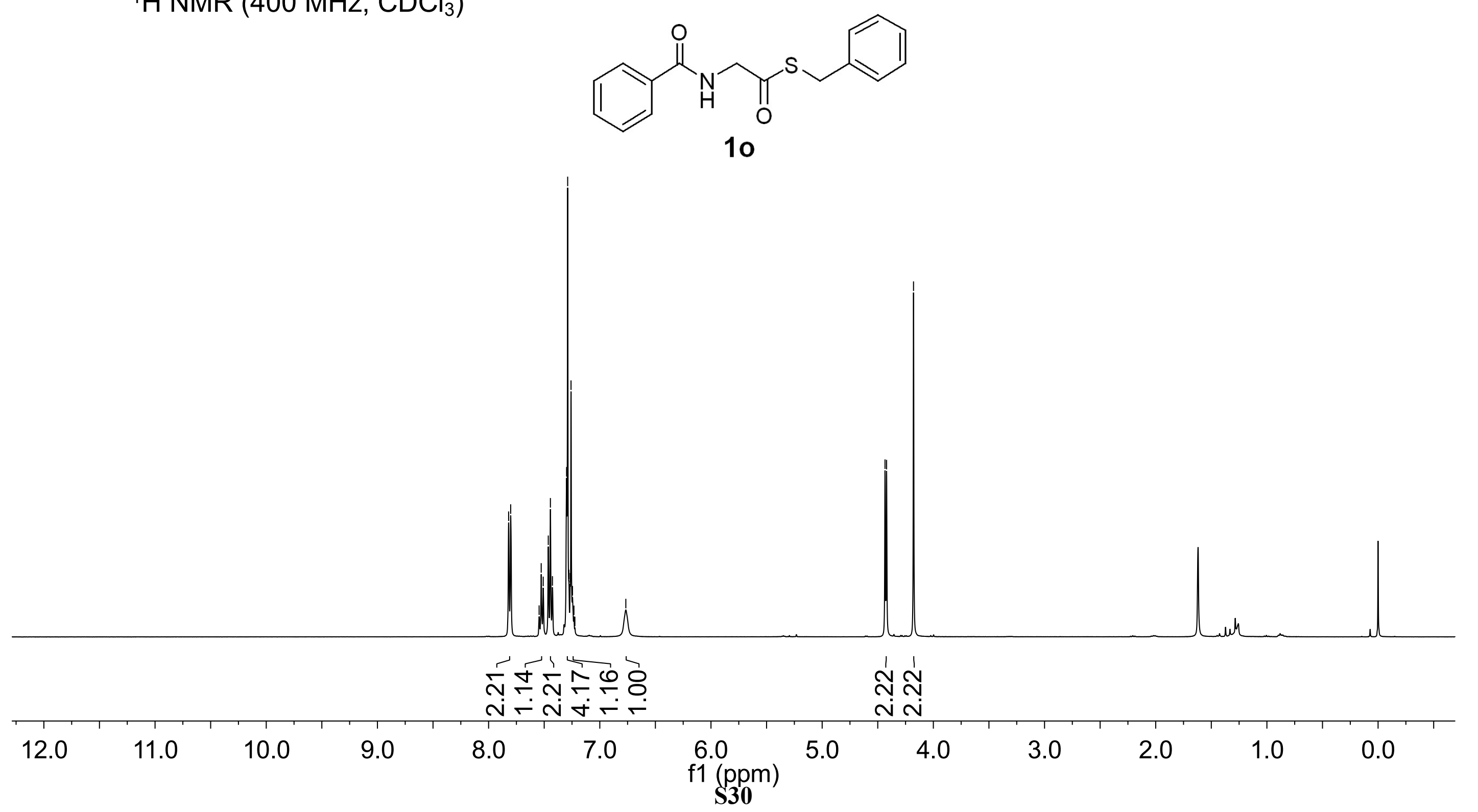
${ }^{13} \mathrm{C}\left\{{ }^{1} \mathrm{H}\right\} \mathrm{NMR}\left(101 \mathrm{MHz}, \mathrm{CDCl}_{3}\right)$<smiles>O=C(CNC(=O)c1ccccc1)SCc1ccccc1</smiles>

\begin{tabular}{|c|c|c|c|c|c|c|c|c|c|c|c|c|c|c|}
\hline 210 & 190 & 170 & 150 & 130 & ${ }_{\mathrm{f} 1}^{11 \text { (ppm) }_{\mathbf{S 3 1}}} 90$ & 80 & 70 & 60 & 50 & 40 & 30 & 20 & 10 & 0 \\
\hline
\end{tabular}


${ }^{1} \mathrm{H}$ NMR $\left(400 \mathrm{MHz}, \mathrm{CDCl}_{3}\right)$<smiles>Cc1ccc(SCNC(=O)c2ccccc2)cc1</smiles>

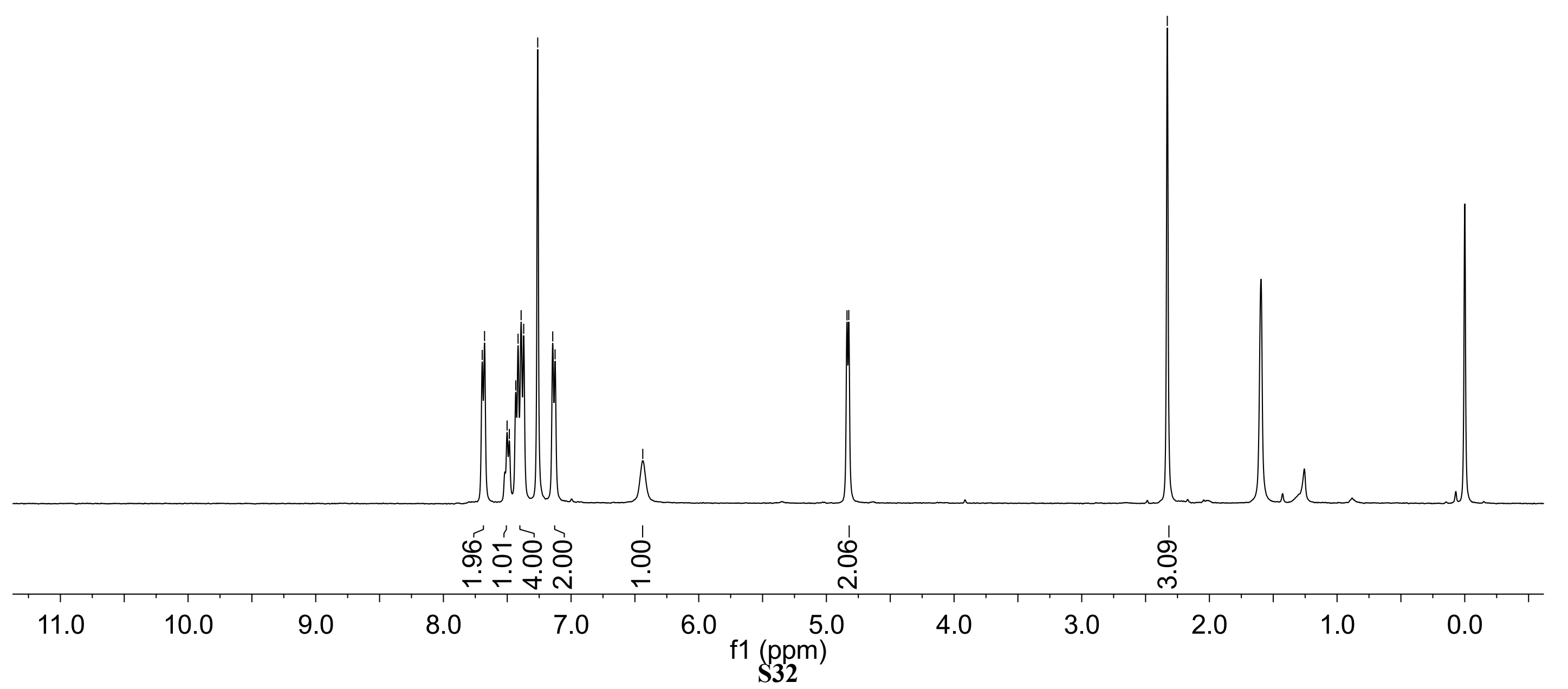


${ }^{13} \mathrm{C}\left\{{ }^{1} \mathrm{H}\right\}$ NMR $\left(101 \mathrm{MHz}, \mathrm{CDCl}_{3}\right)$<smiles>Cc1ccc(SCNC(=O)c2ccccc2)cc1</smiles>

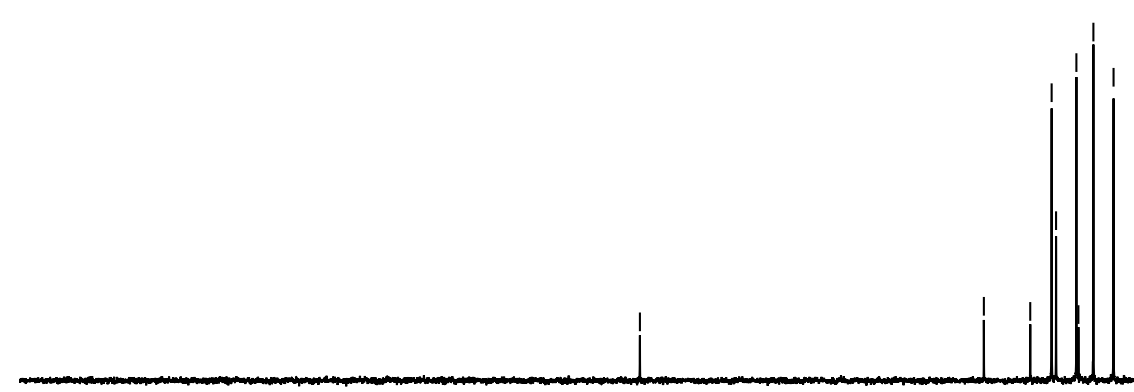

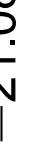

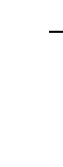

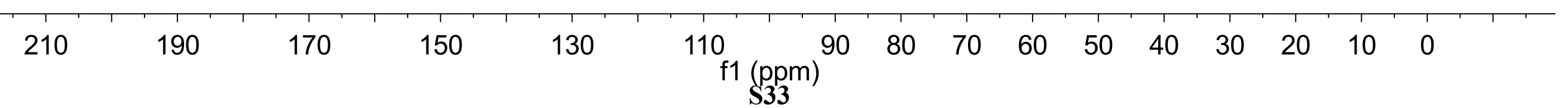




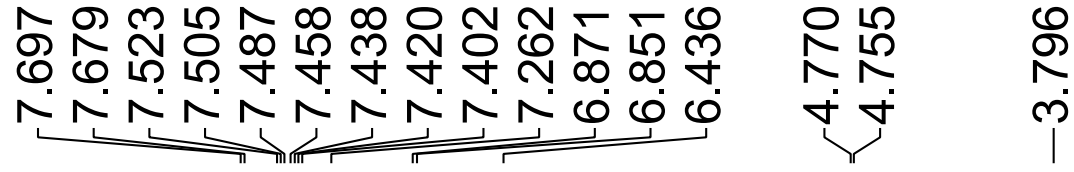

${ }^{1} \mathrm{H}$ NMR $\left(400 \mathrm{MHz}, \mathrm{CDCl}_{3}\right)$

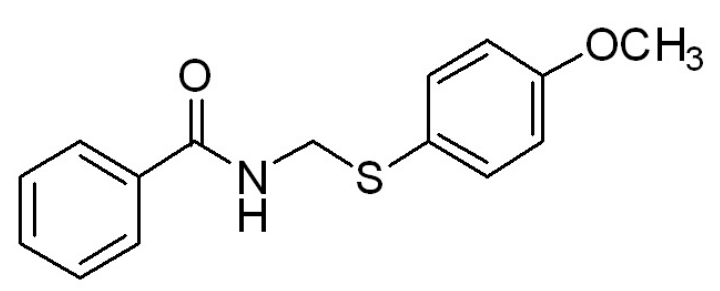

2b

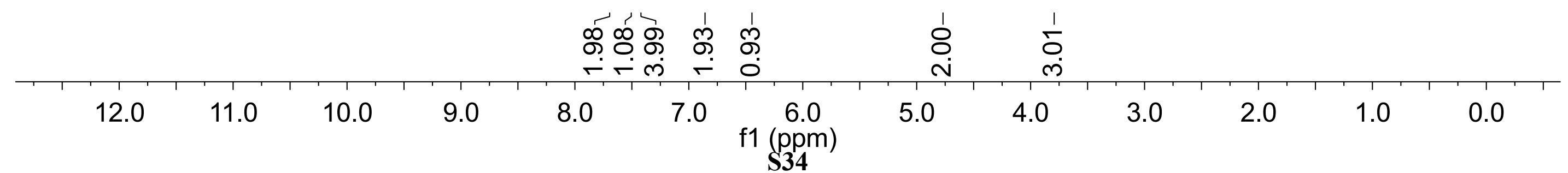


${ }^{13} \mathrm{C}\left\{{ }^{1} \mathrm{H}\right\}$ NMR $\left(101 \mathrm{MHz}, \mathrm{CDCl}_{3}\right)$<smiles>COc1ccc(SCNC(=O)c2ccccc2)cc1</smiles>

2b

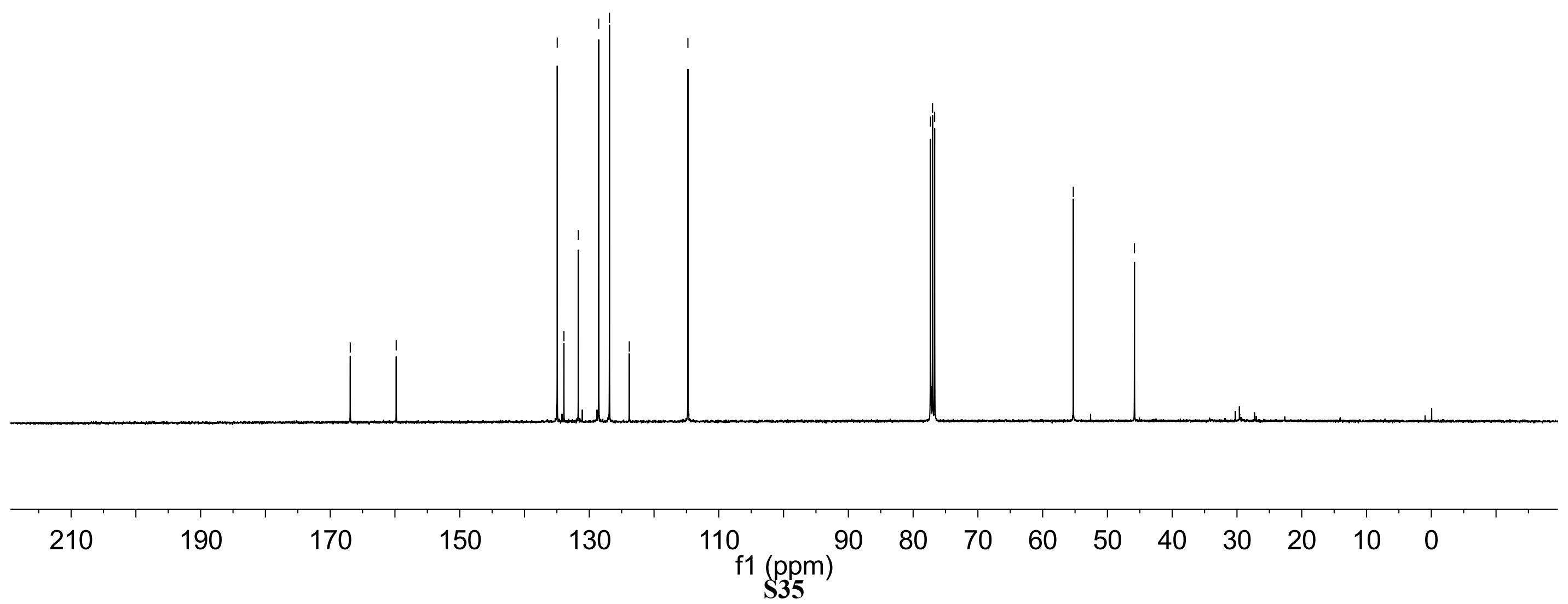


${ }^{1} \mathrm{H}$ NMR $\left(400 \mathrm{MHz}, \mathrm{CDCl}_{3}\right)$
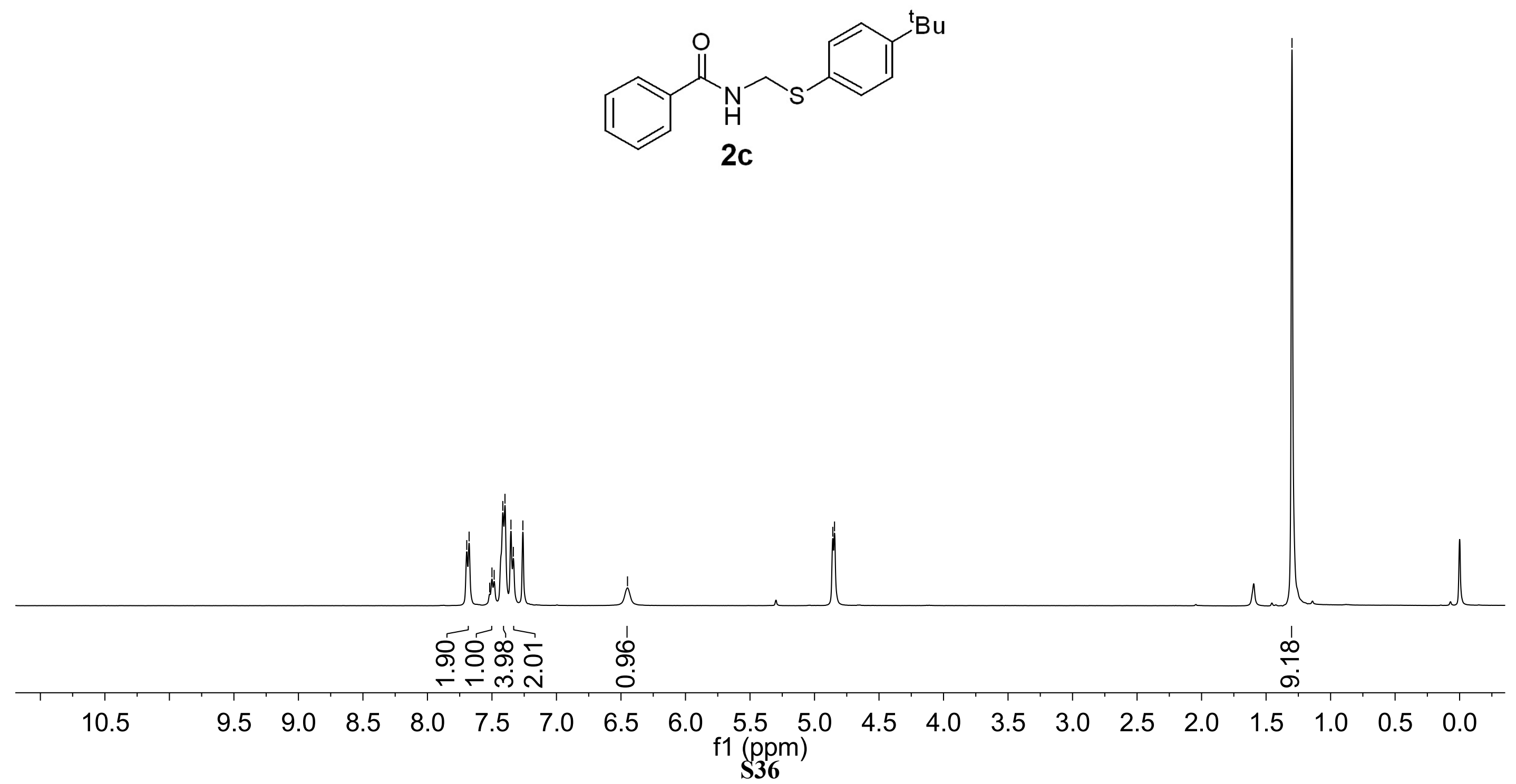
${ }^{13} \mathrm{C}\left\{{ }^{1} \mathrm{H}\right\}$ NMR $\left(101 \mathrm{MHz}, \mathrm{CDCl}_{3}\right)$<smiles>CC(C)(C)c1ccc(SCNC(=O)c2ccccc2)cc1</smiles>
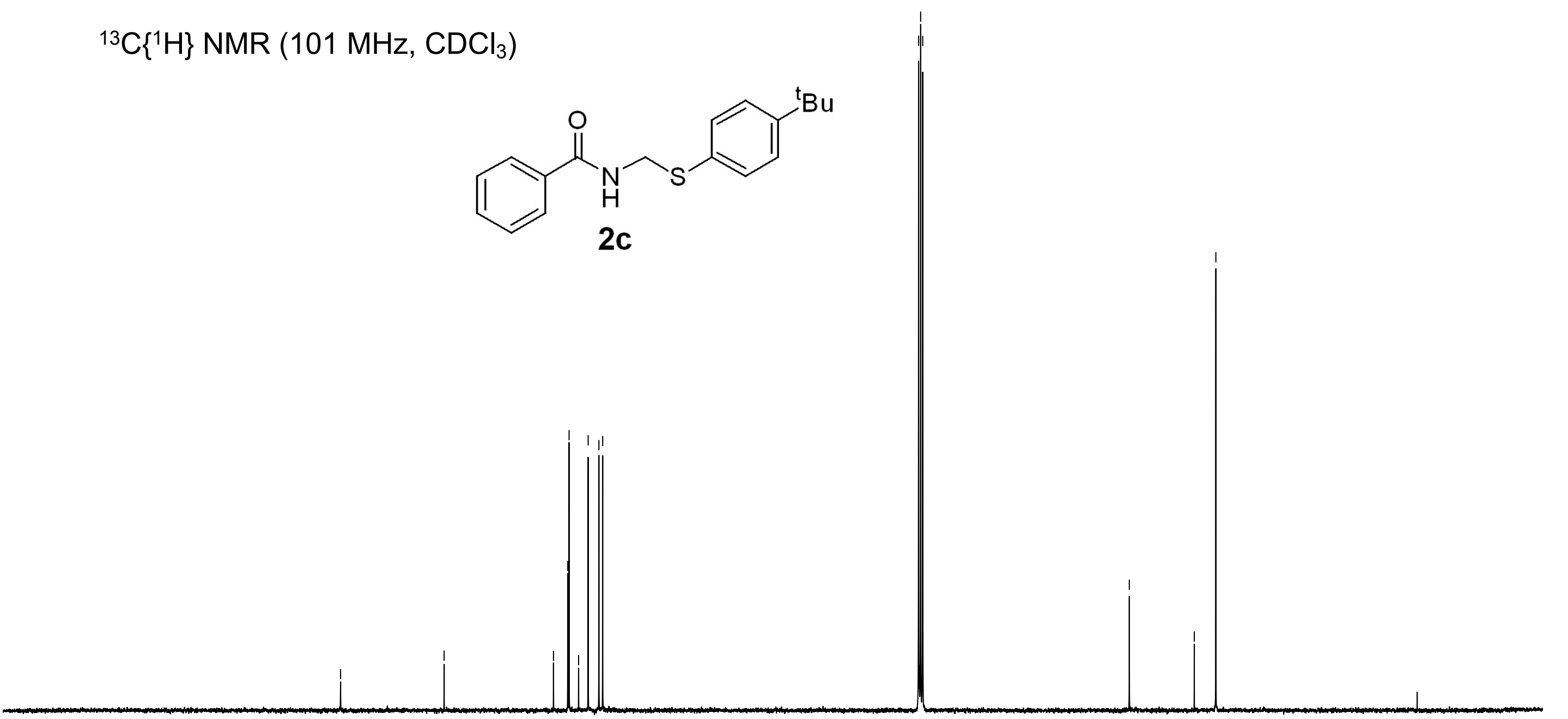

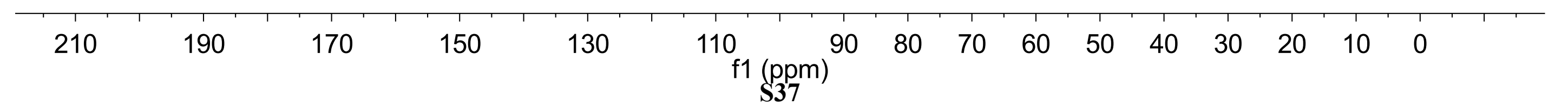


${ }^{1} \mathrm{H}$ NMR $\left(400 \mathrm{MHz}, \mathrm{CDCl}_{3}\right)$
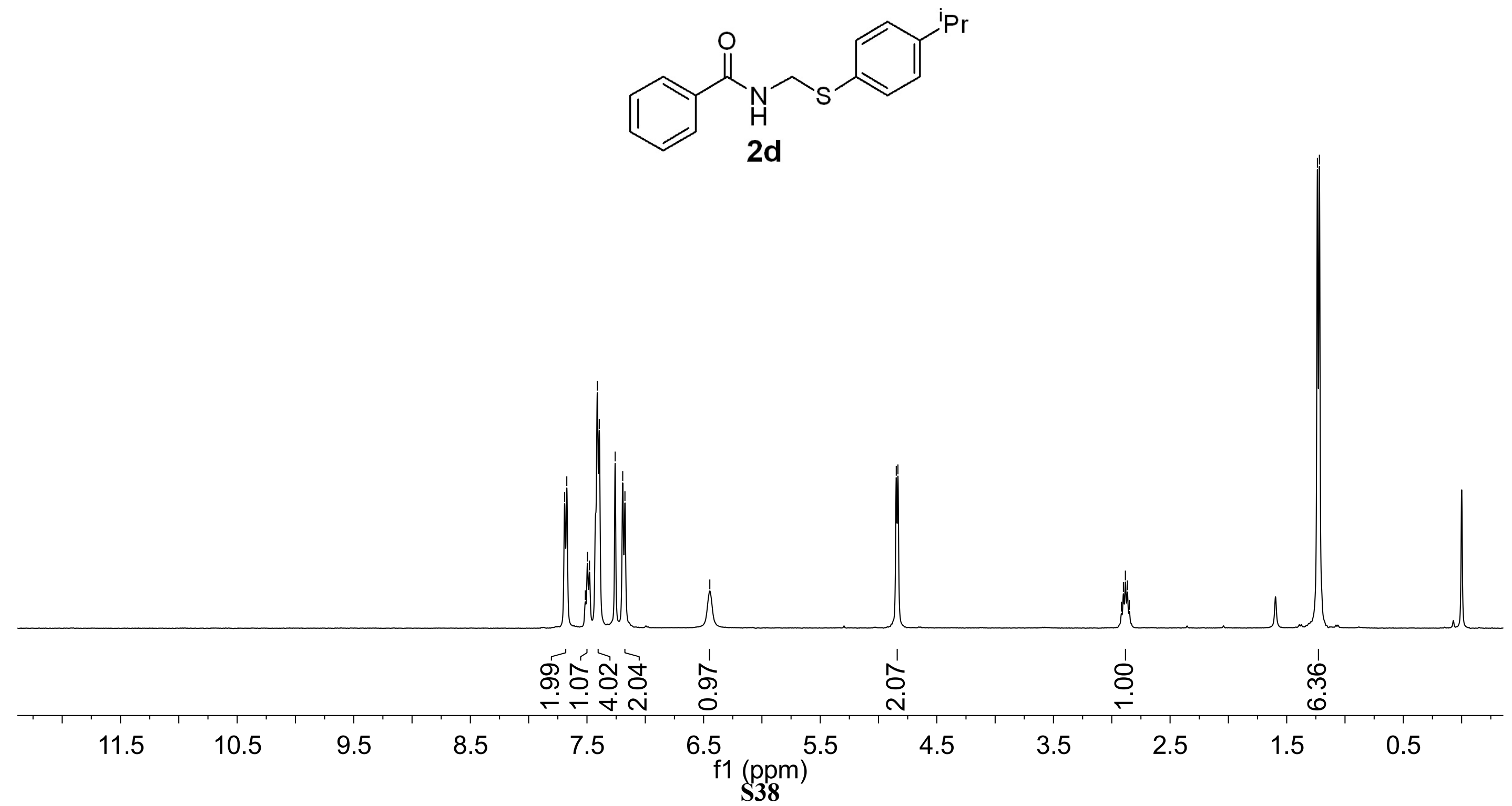
${ }^{13} \mathrm{C}\left\{{ }^{1} \mathrm{H}\right\}$ NMR $\left(101 \mathrm{MHz}, \mathrm{CDCl}_{3}\right)$

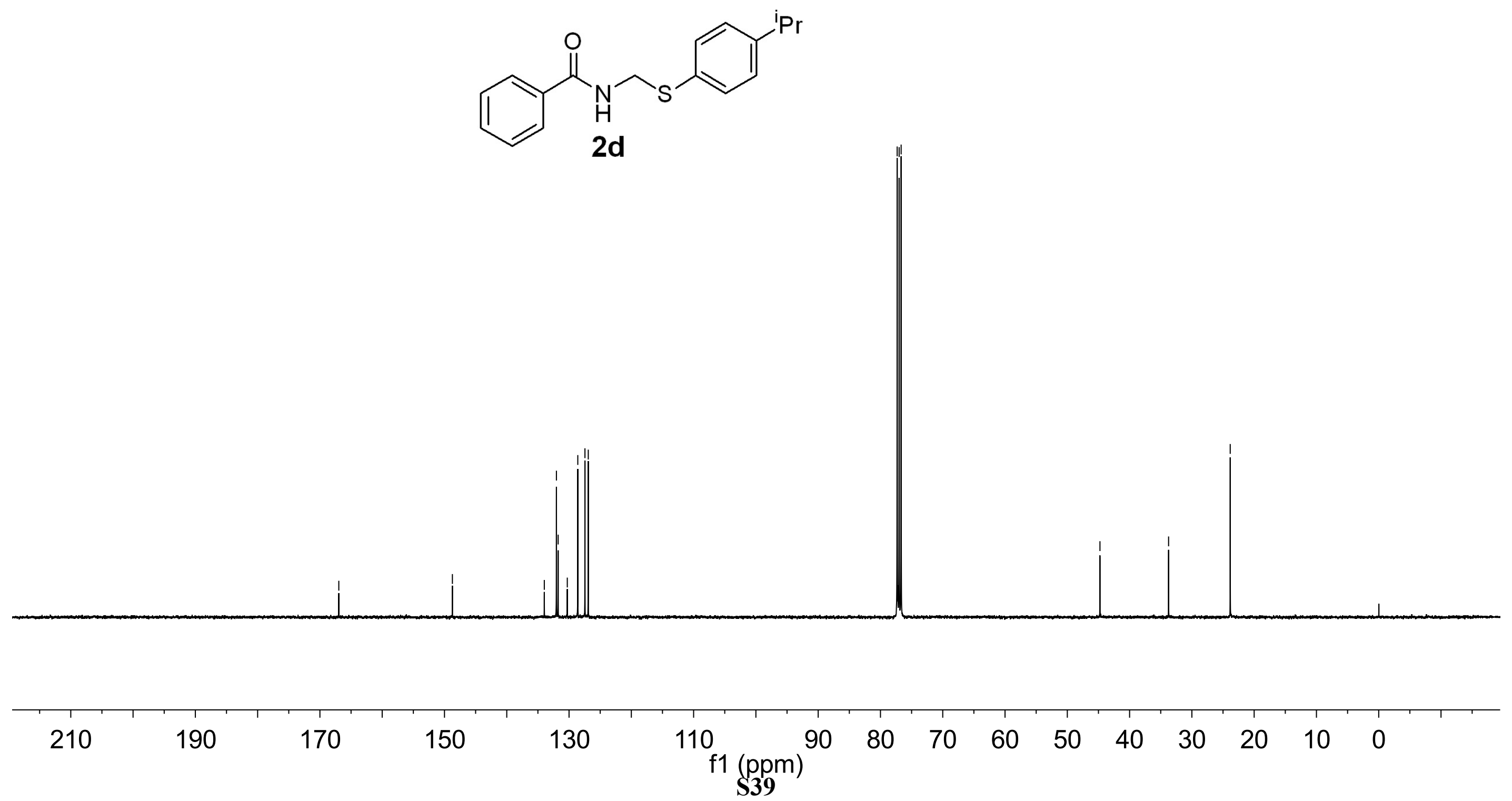


${ }^{1} \mathrm{H}$ NMR $\left(400 \mathrm{MHz}, \mathrm{CDCl}_{3}\right)$<smiles>Cc1ccccc1SCNC(=O)c1ccccc1</smiles>

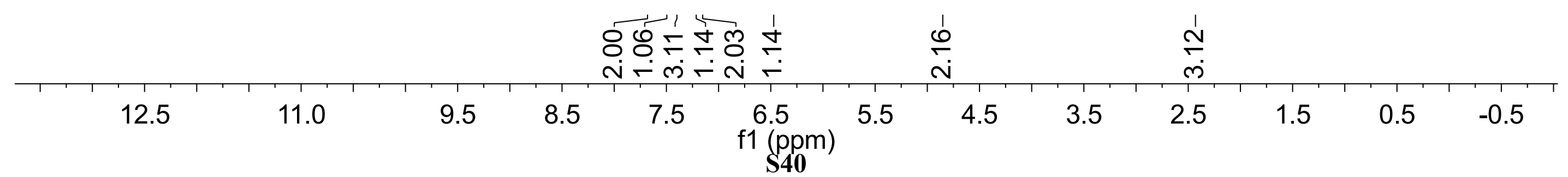


${ }^{13} \mathrm{C}\left\{{ }^{1} \mathrm{H}\right\} \operatorname{NMR}\left(101 \mathrm{MHz}, \mathrm{CDCl}_{3}\right)$<smiles>Cc1ccccc1SCNC(=O)c1ccccc1</smiles>

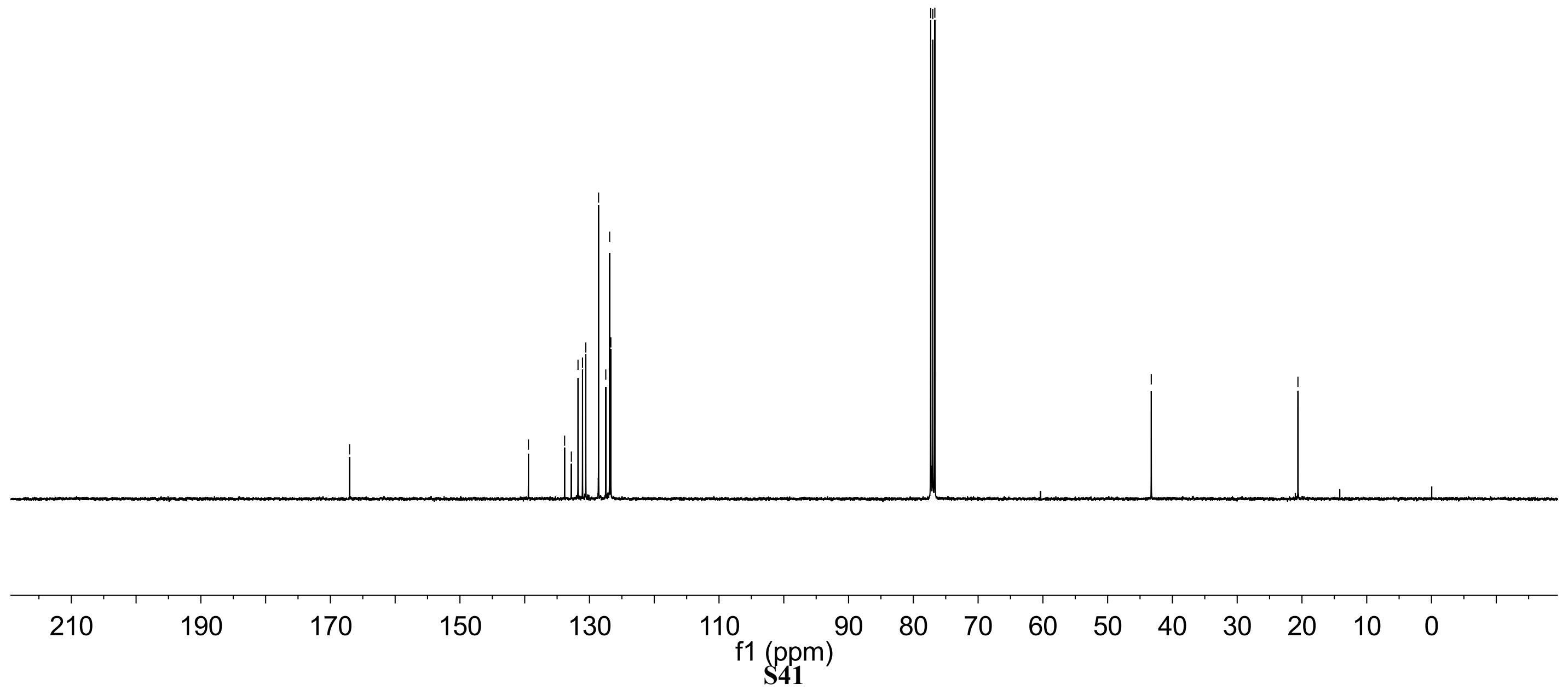




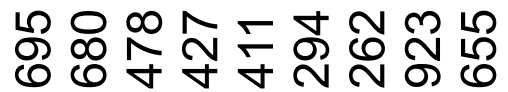 \\ N N N N

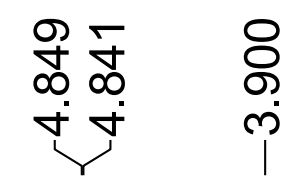

${ }^{1} \mathrm{H}$ NMR $\left(400 \mathrm{MHz}, \mathrm{CDCl}_{3}\right)$
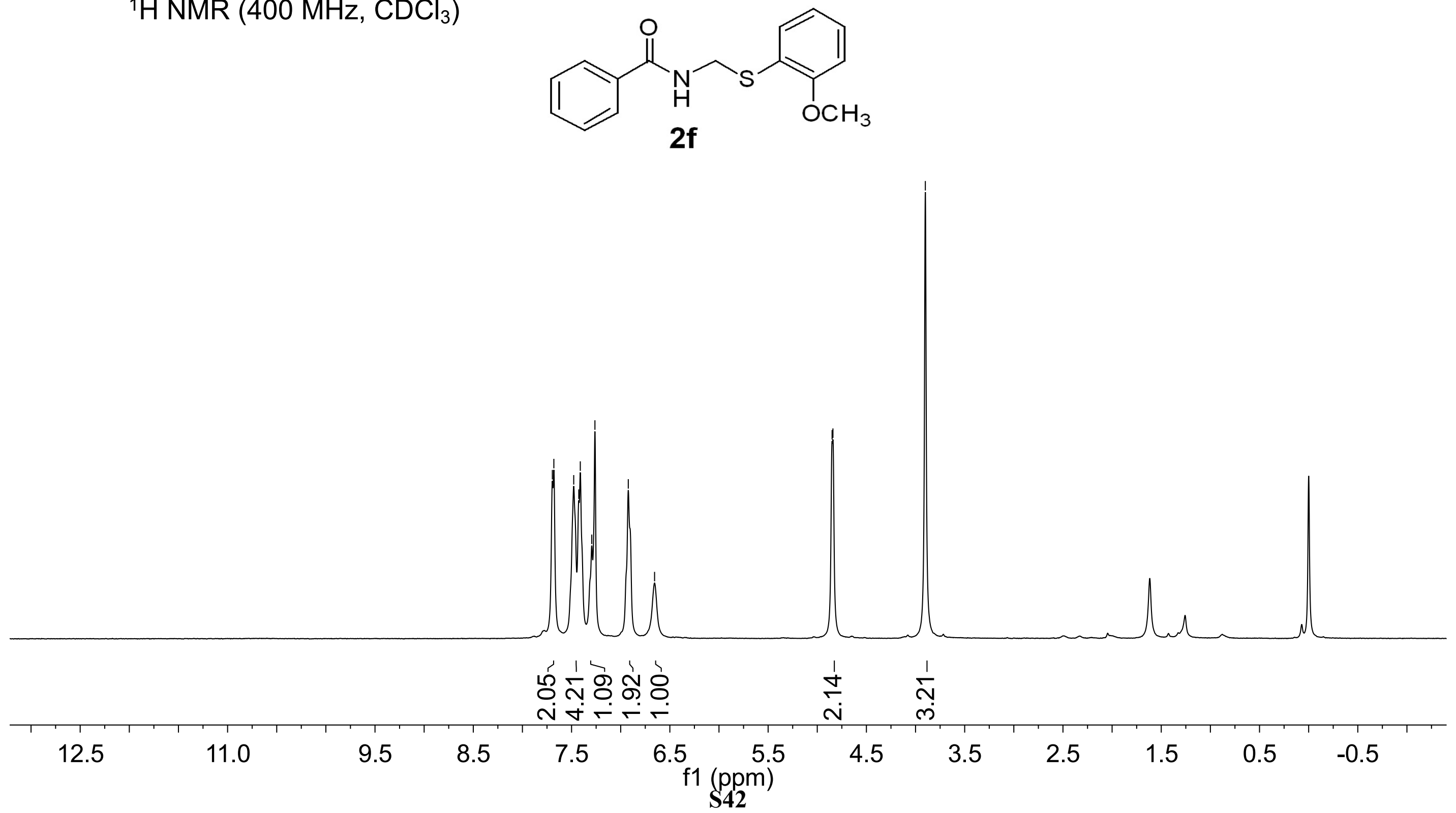


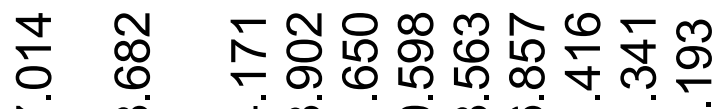

은 욤

๓ $8 \%$

NN้

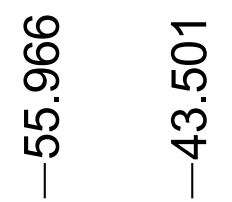

${ }^{13} \mathrm{C}\left\{{ }^{1} \mathrm{H}\right\}$ NMR (101 MHz, $\left.\mathrm{CDCl}_{3}\right)$<smiles>CCCCSc1ccccc1OC</smiles>

舟 
${ }^{1} \mathrm{H}$ NMR $\left(400 \mathrm{MHz}, \mathrm{CDCl}_{3}\right)$<smiles>O=C(NCSc1ccc2ccccc2c1)c1ccccc1</smiles>

$2 g$

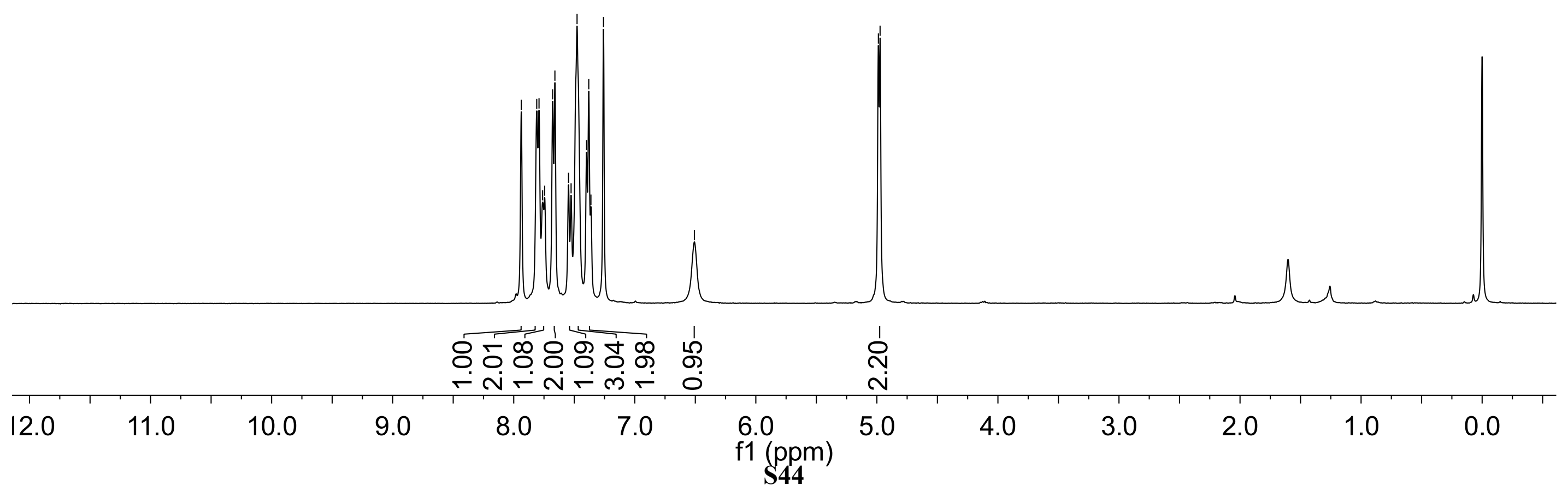


${ }^{13} \mathrm{C}\left\{{ }^{1} \mathrm{H}\right\}$ NMR $\left(101 \mathrm{MHz}, \mathrm{CDCl}{ }_{3}\right)$<smiles>O=C(NCSc1ccc2ccccc2c1)c1ccccc1</smiles>

$2 g$

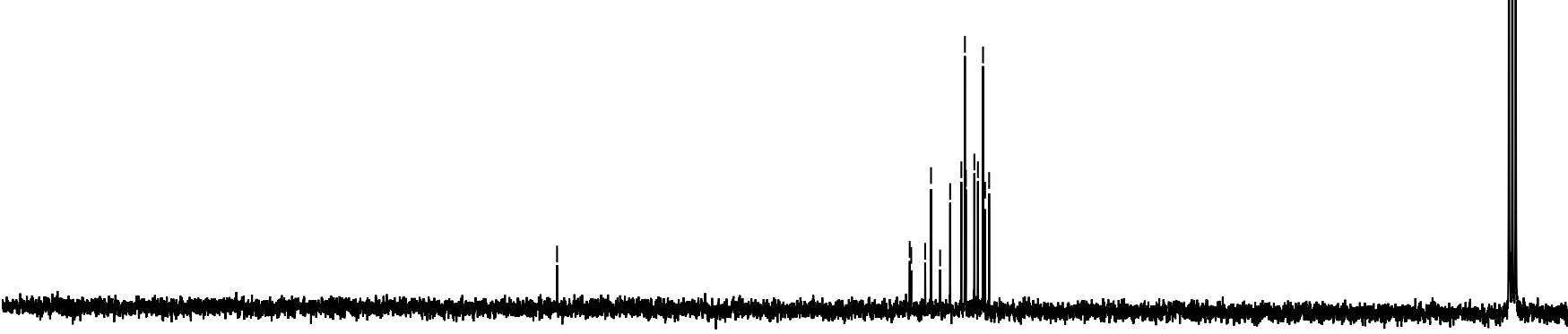

\begin{tabular}{lllllllllllllllll}
\hline 210 & 190 & 170 & 150 & 130 & $\begin{array}{c}110 \\
\mathrm{f1}(\mathrm{ppm})\end{array}$ & 90 & 80 & 70 & 60 & 50 & 40 & 30 & 20 & 10 & 0 &
\end{tabular}




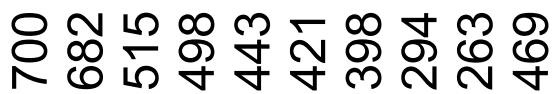

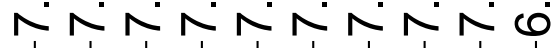

${ }^{1} \mathrm{H}$ NMR $\left(400 \mathrm{MHz}, \mathrm{CDCl}_{3}\right)$
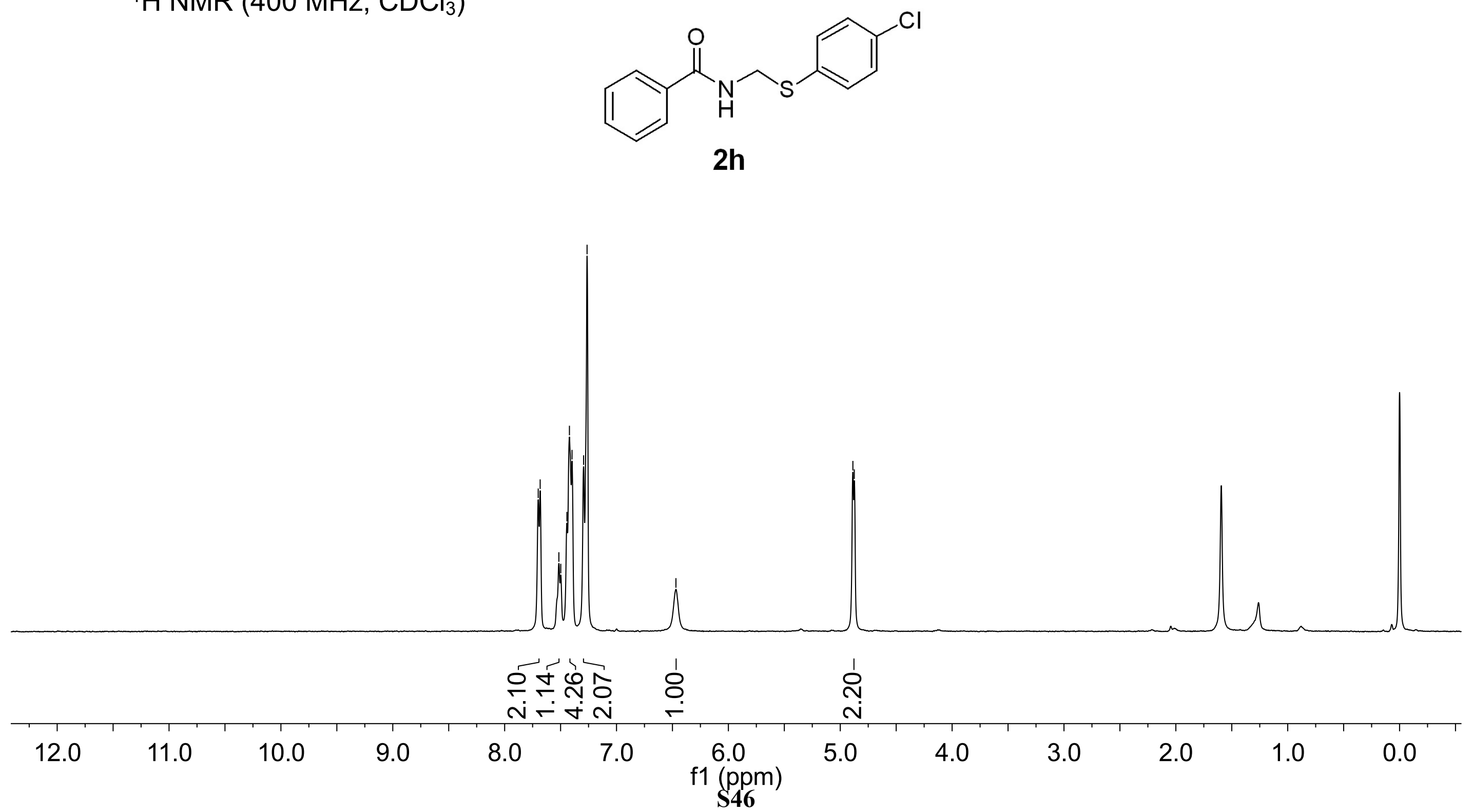
${ }^{13} \mathrm{C}\left\{{ }^{1} \mathrm{H}\right\} \operatorname{NMR}\left(101 \mathrm{MHz}, \mathrm{CDCl}_{3}\right)$<smiles>O=C(NCSc1ccc(Cl)cc1)c1ccccc1</smiles>

\section{$2 \mathrm{~h}$}


${ }^{1} \mathrm{H} \mathrm{NMR}\left(400 \mathrm{MHz}, \mathrm{CDCl}_{3}\right)$
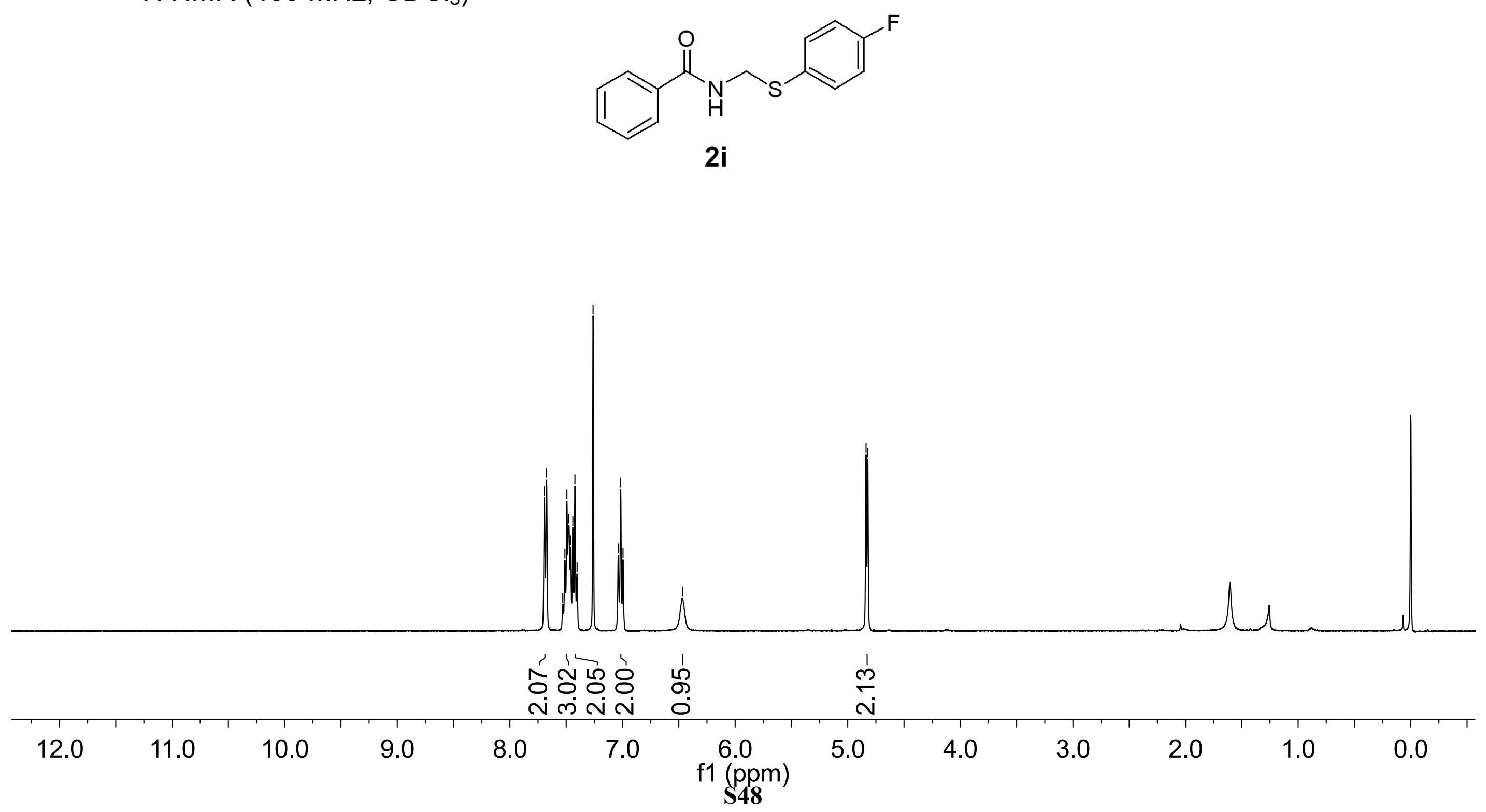
${ }^{13} \mathrm{C}\left\{{ }^{1} \mathrm{H}\right\}$ NMR (101 MHz, $\left.\mathrm{CDCl}_{3}\right)$<smiles>O=C(NCSc1ccc(F)cc1)c1ccccc1</smiles>

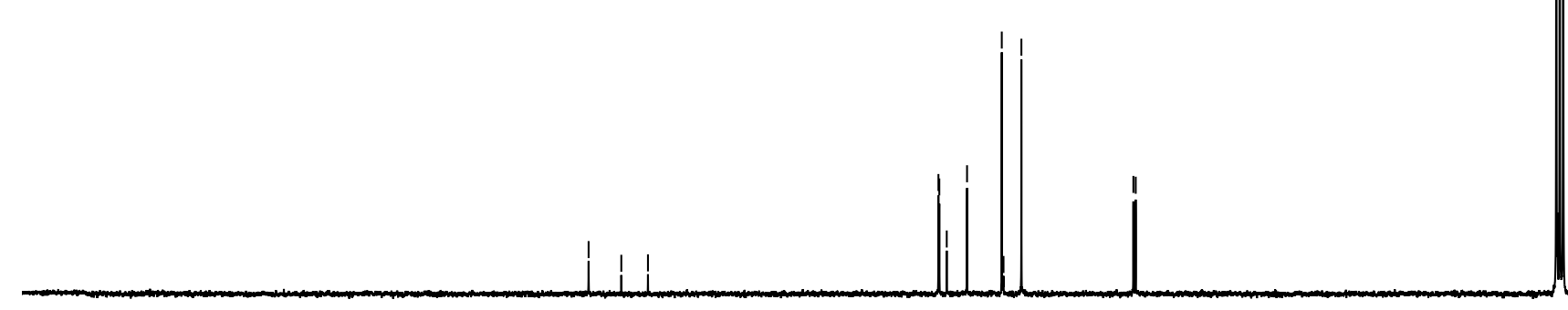

\begin{tabular}{llllllllllllllllll}
\hline 210 & 190 & 170 & 150 & 130 & $\begin{array}{c}110 \\
\mathrm{f1}(\mathrm{ppm})\end{array}$ & 90 & 80 & 70 & 60 & 50 & 40 & 30 & 20 & 10 & 0 &
\end{tabular}


${ }^{1} \mathrm{H}$ NMR $\left(400 \mathrm{MHz}, \mathrm{CDCl}_{3}\right)$
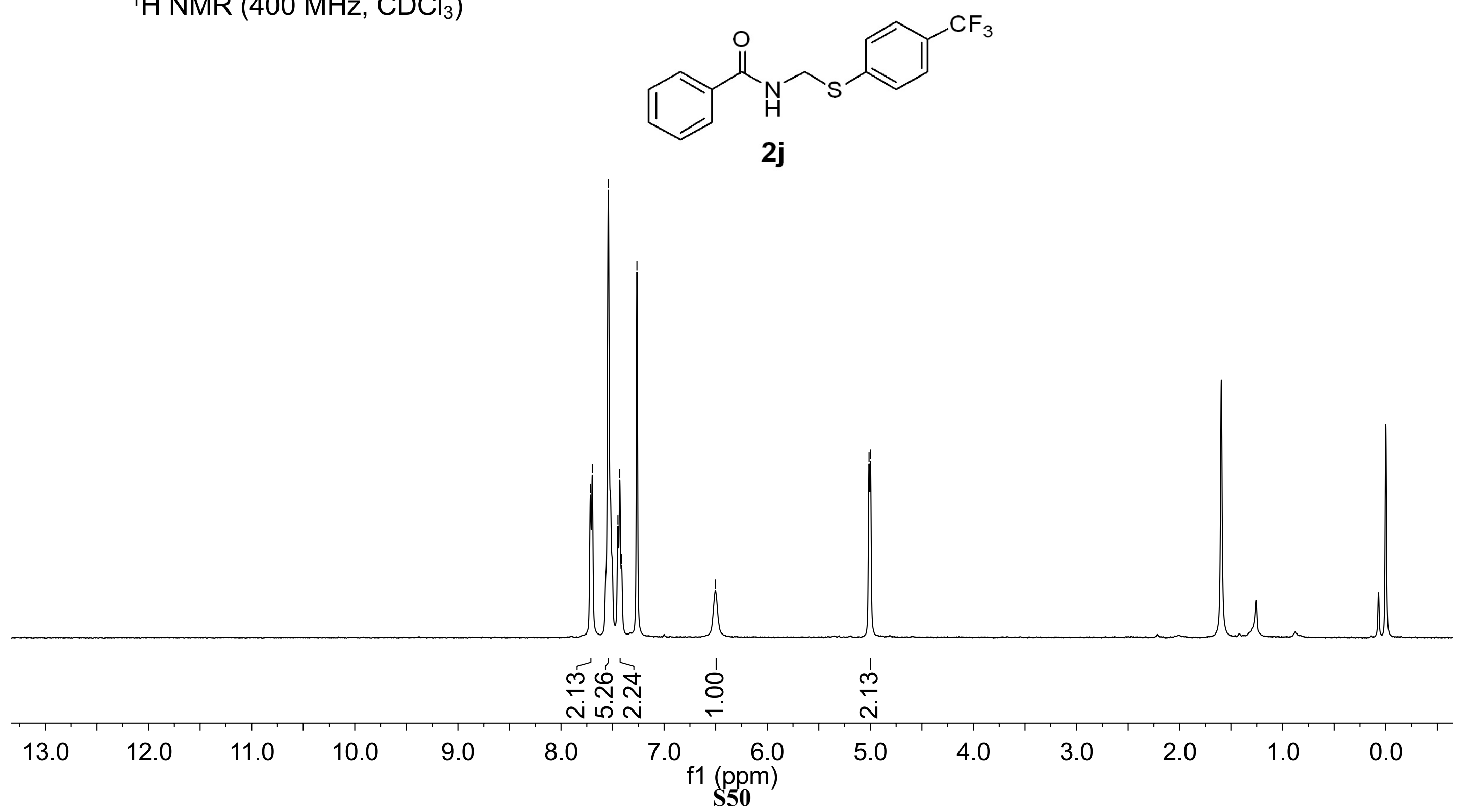
${ }^{13} \mathrm{C}\left\{{ }^{1} \mathrm{H}\right\}$ NMR $\left(101 \mathrm{MHz}, \mathrm{CDCl}_{3}\right)$

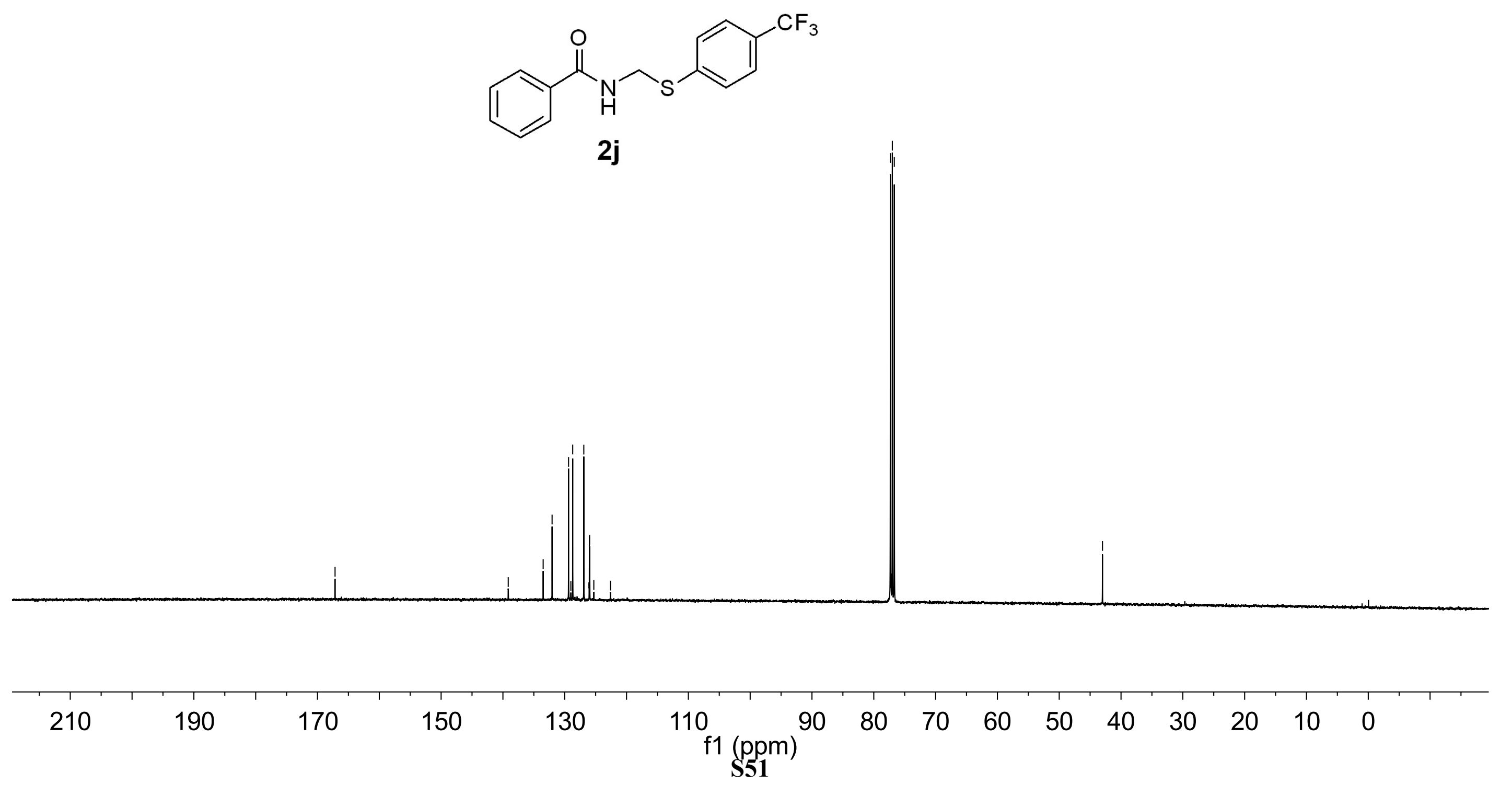




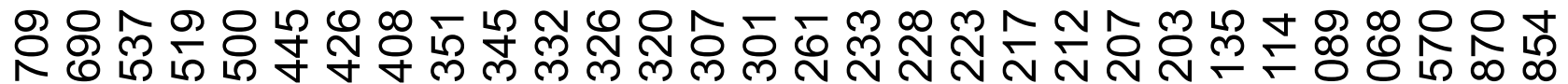

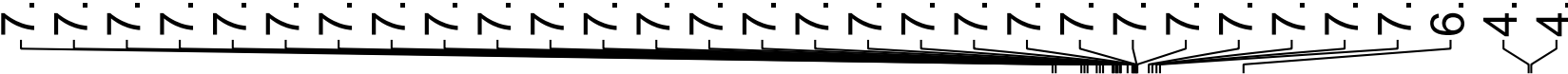

${ }^{1} \mathrm{H} \mathrm{NMR}\left(400 \mathrm{MHz}, \mathrm{CDCl}_{3}\right)$
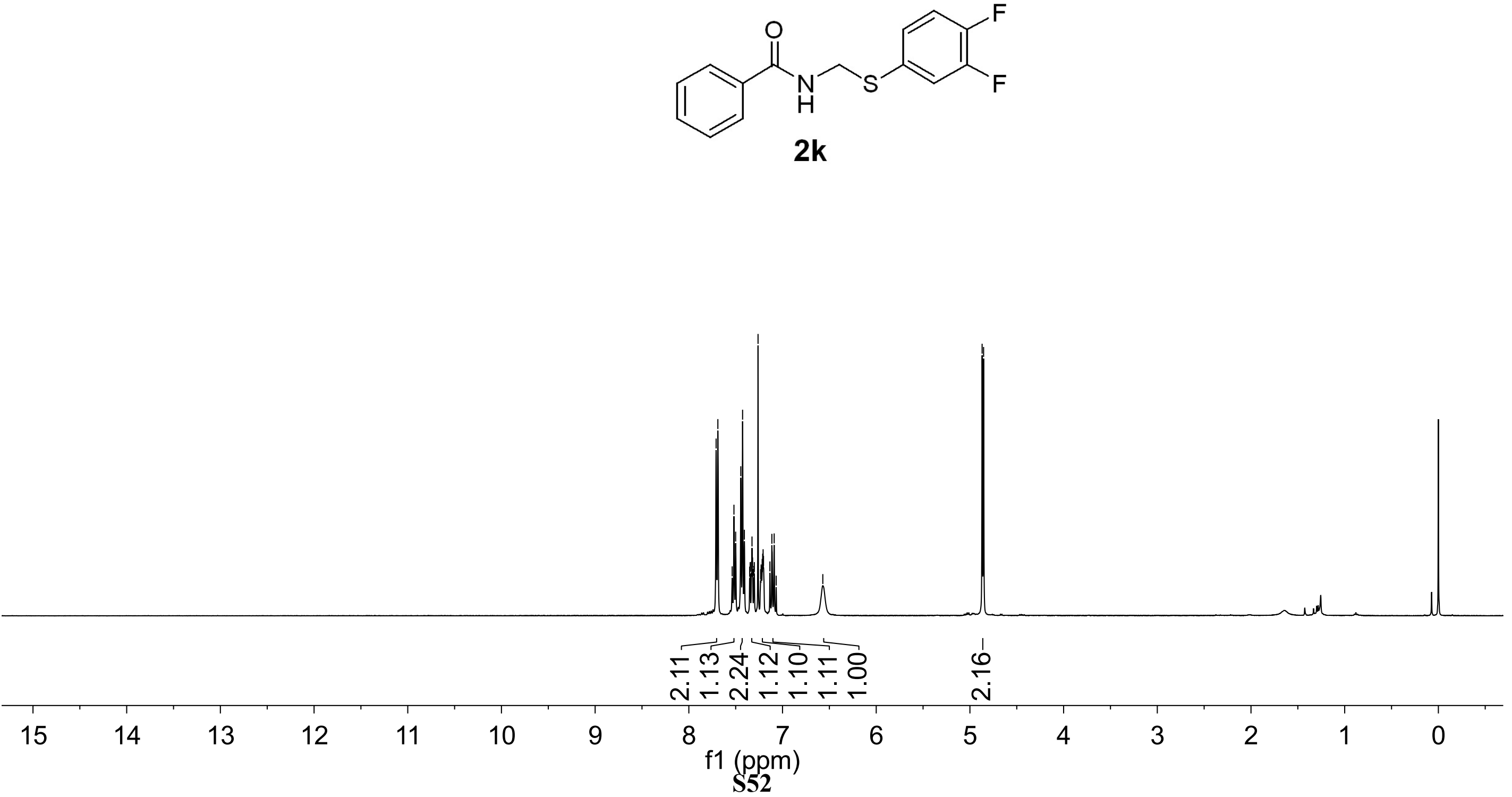


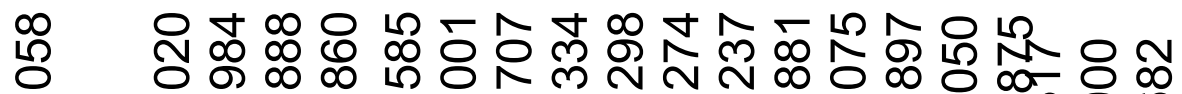

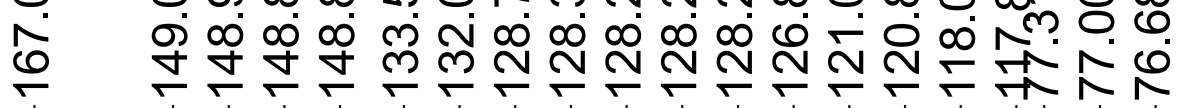

${ }^{13} \mathrm{C}\left\{{ }^{1} \mathrm{H}\right\} \operatorname{NMR}\left(101 \mathrm{MHz}, \mathrm{CDCl}_{3}\right)$
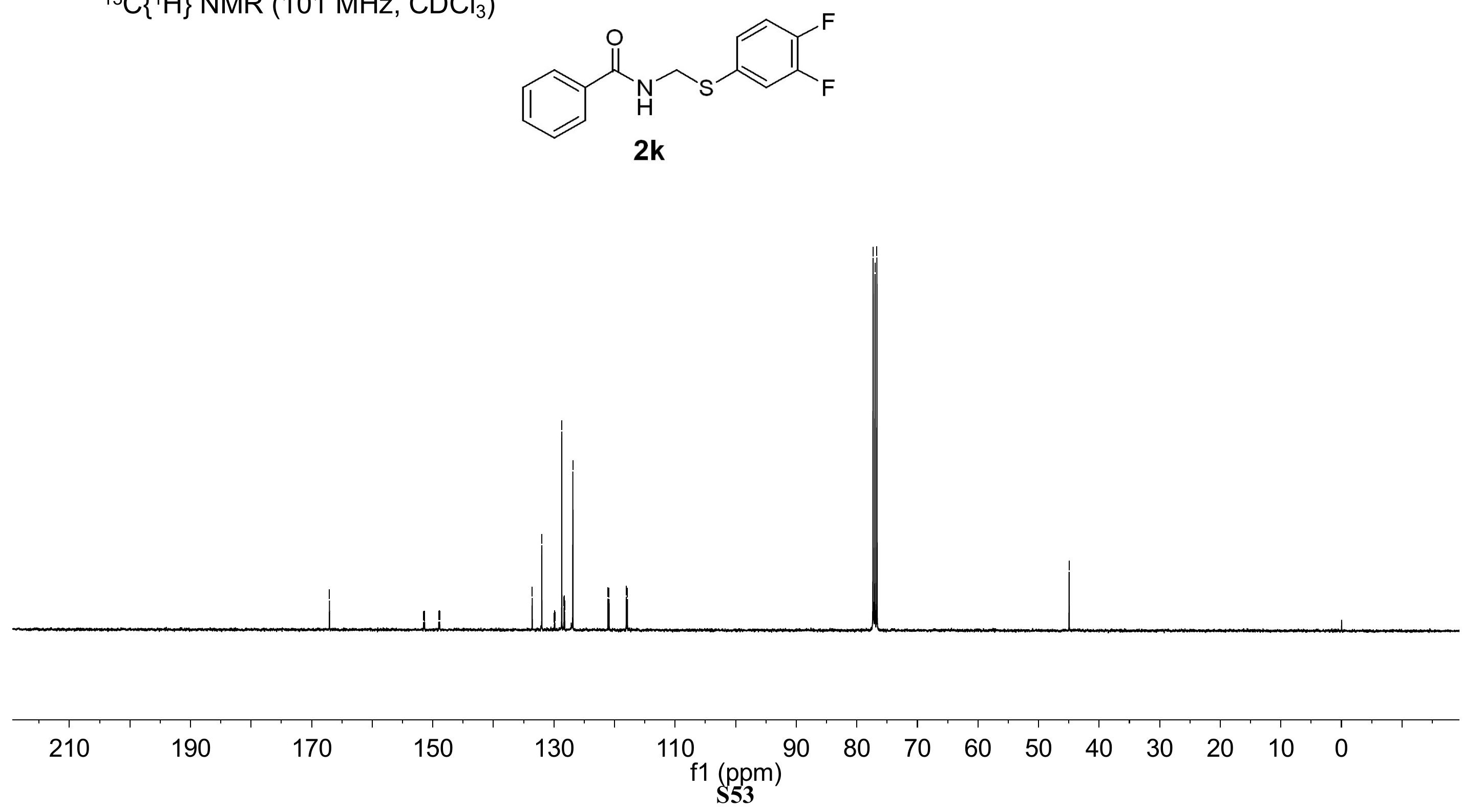
${ }^{1} \mathrm{H}$ NMR $\left(400 \mathrm{MHz}, \mathrm{CDCl}_{3}\right)$
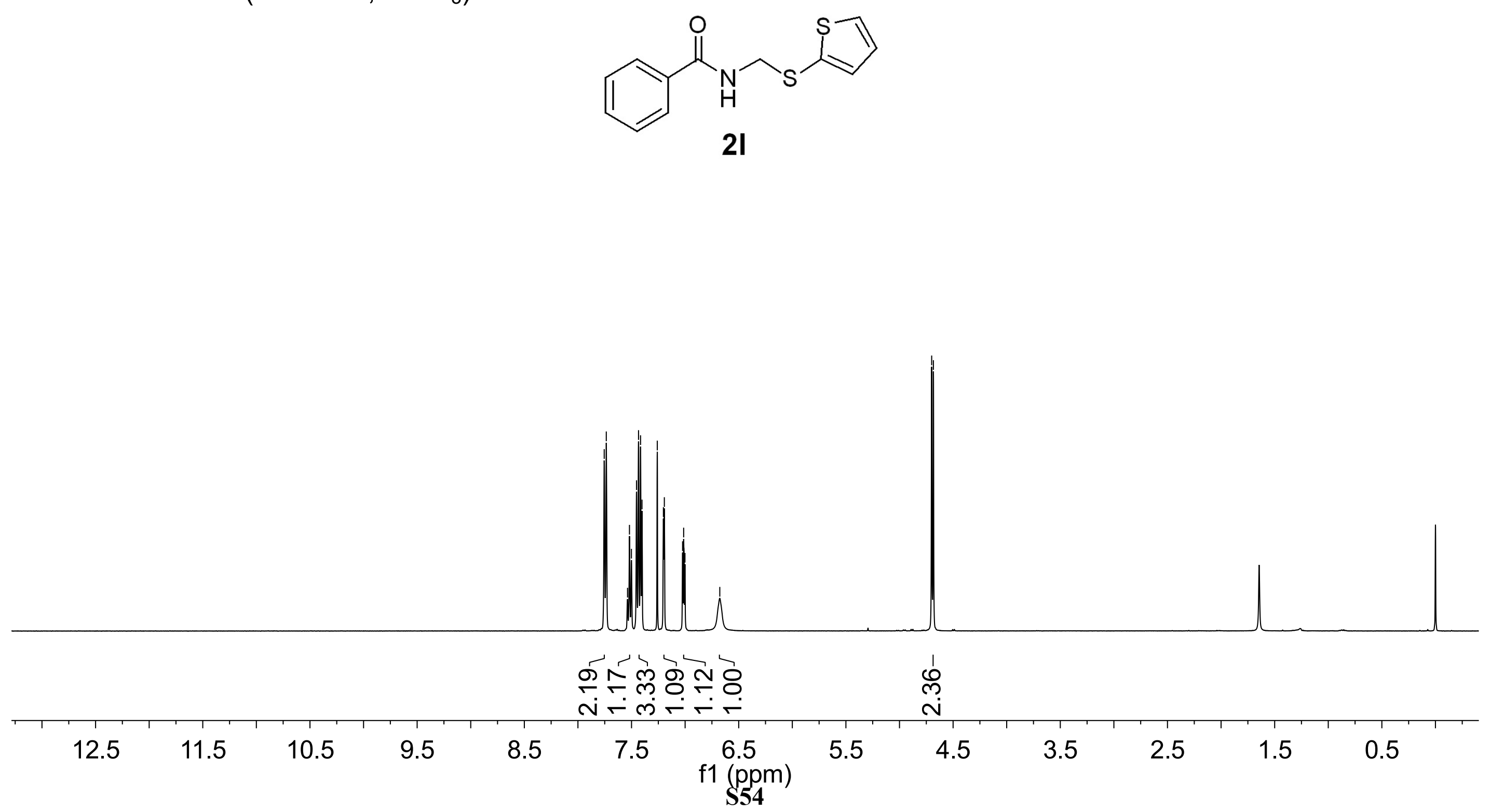
${ }^{13} \mathrm{C}\left\{{ }^{1} \mathrm{H}\right\}$ NMR $\left(101 \mathrm{MHz}, \mathrm{CDCl}_{3}\right)$<smiles>O=C(NCSc1cccs1)c1ccccc1</smiles>

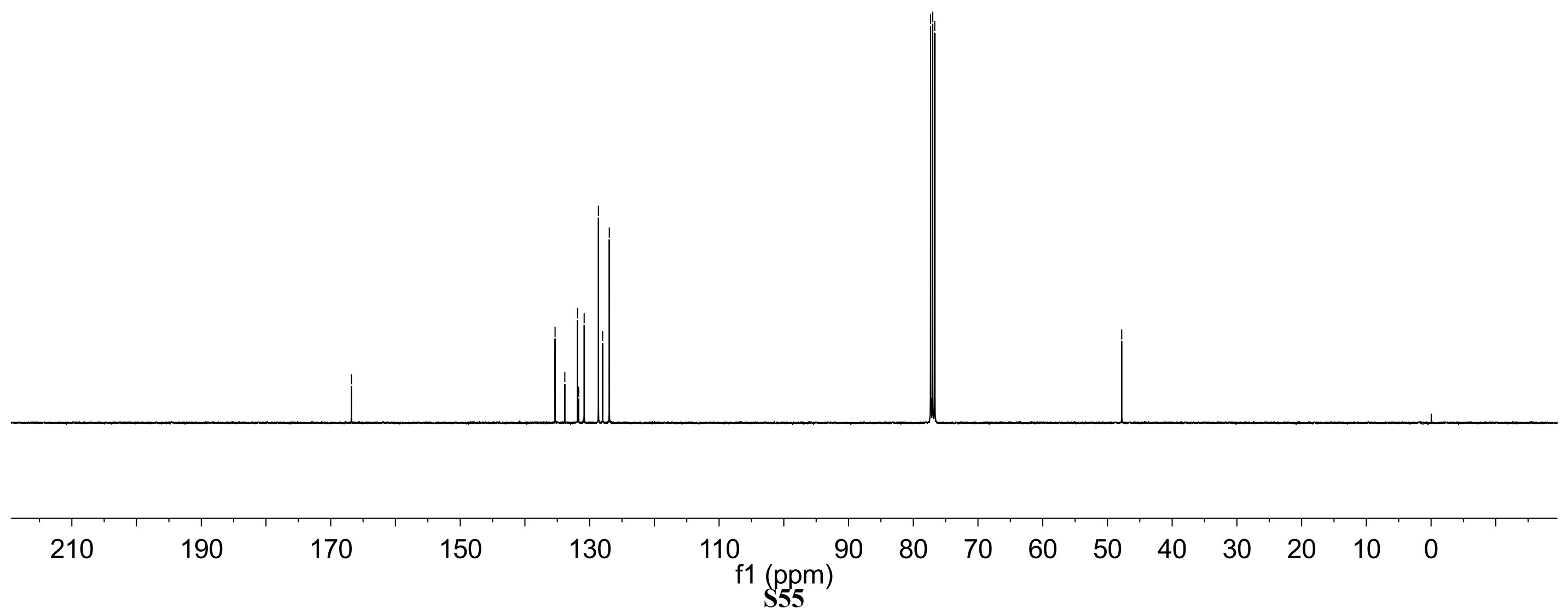


${ }^{1} \mathrm{H} \mathrm{NMR}\left(400 \mathrm{MHz}, \mathrm{CDCl}_{3}\right)$<smiles>Cc1occc1SCNC(=O)c1ccccc1</smiles>

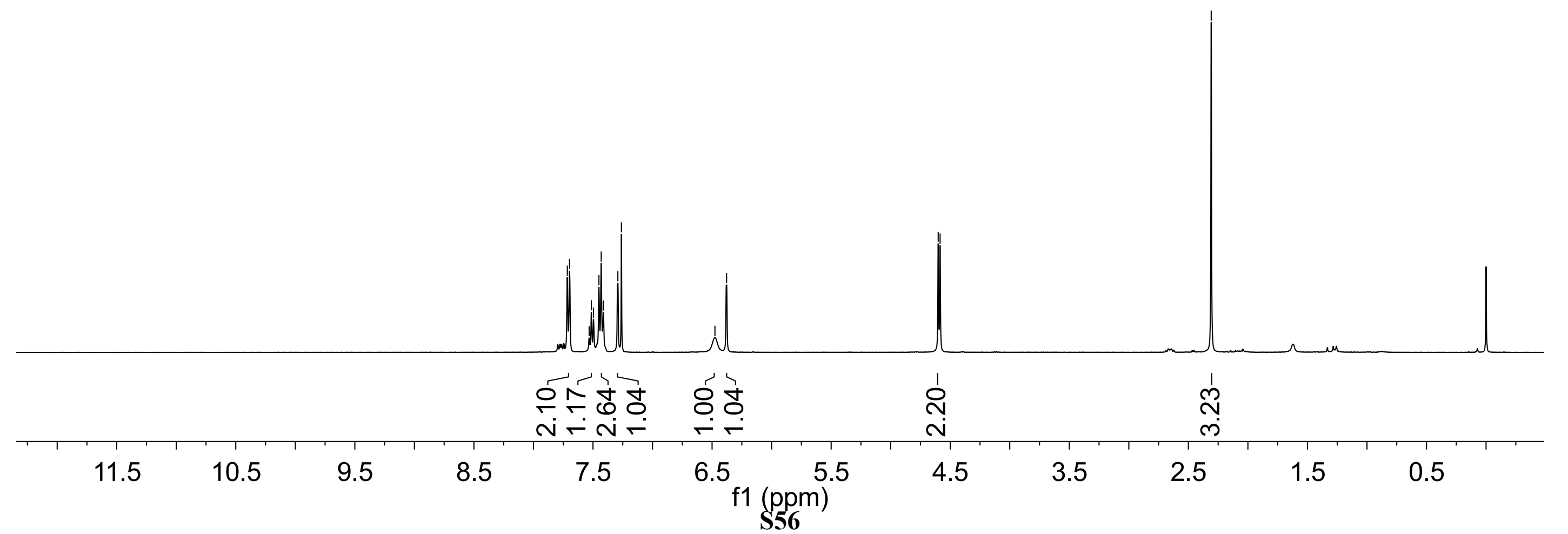


${ }^{13} \mathrm{C}\left\{{ }^{1} \mathrm{H}\right\}$ NMR $\left(101 \mathrm{MHz}, \mathrm{CDCl}_{3}\right)$
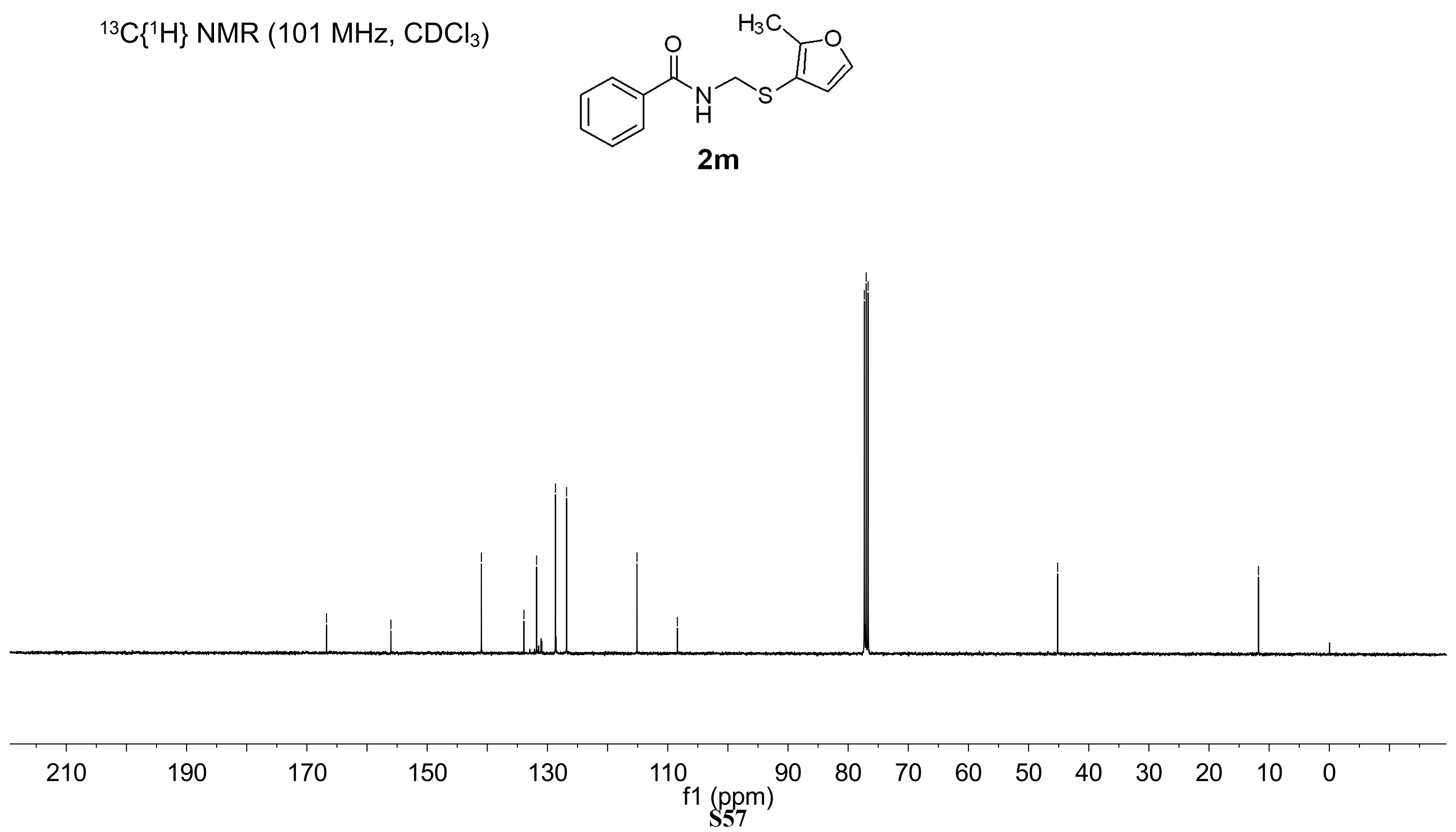
${ }^{1} \mathrm{H}$ NMR $\left(400 \mathrm{MHz}, \mathrm{CDCl}_{3}\right)$<smiles>CC(C)SCNC(=O)c1ccccc1</smiles>

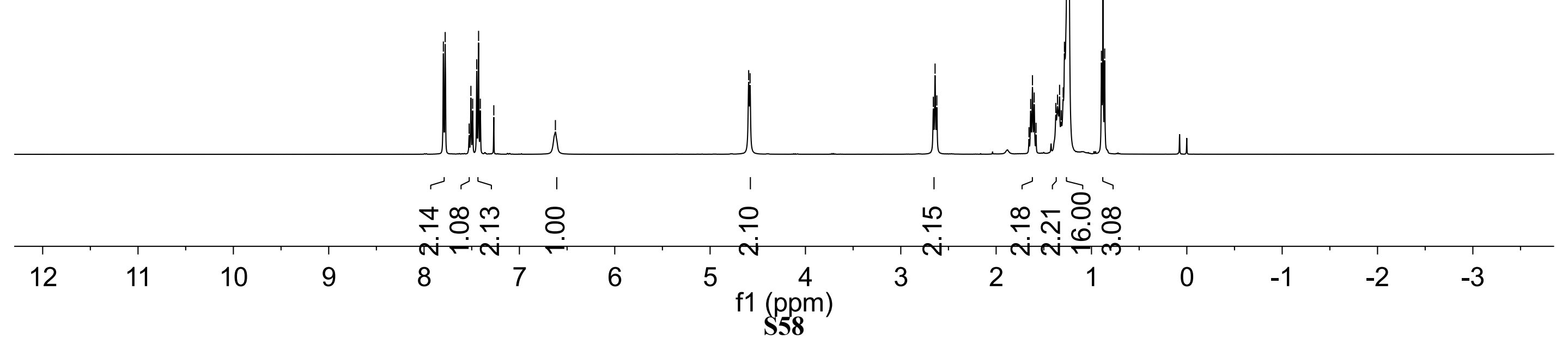


${ }^{13} \mathrm{C}\left\{{ }^{1} \mathrm{H}\right\}$ NMR $\left(101 \mathrm{MHz}, \mathrm{CDCl}_{3}\right)$
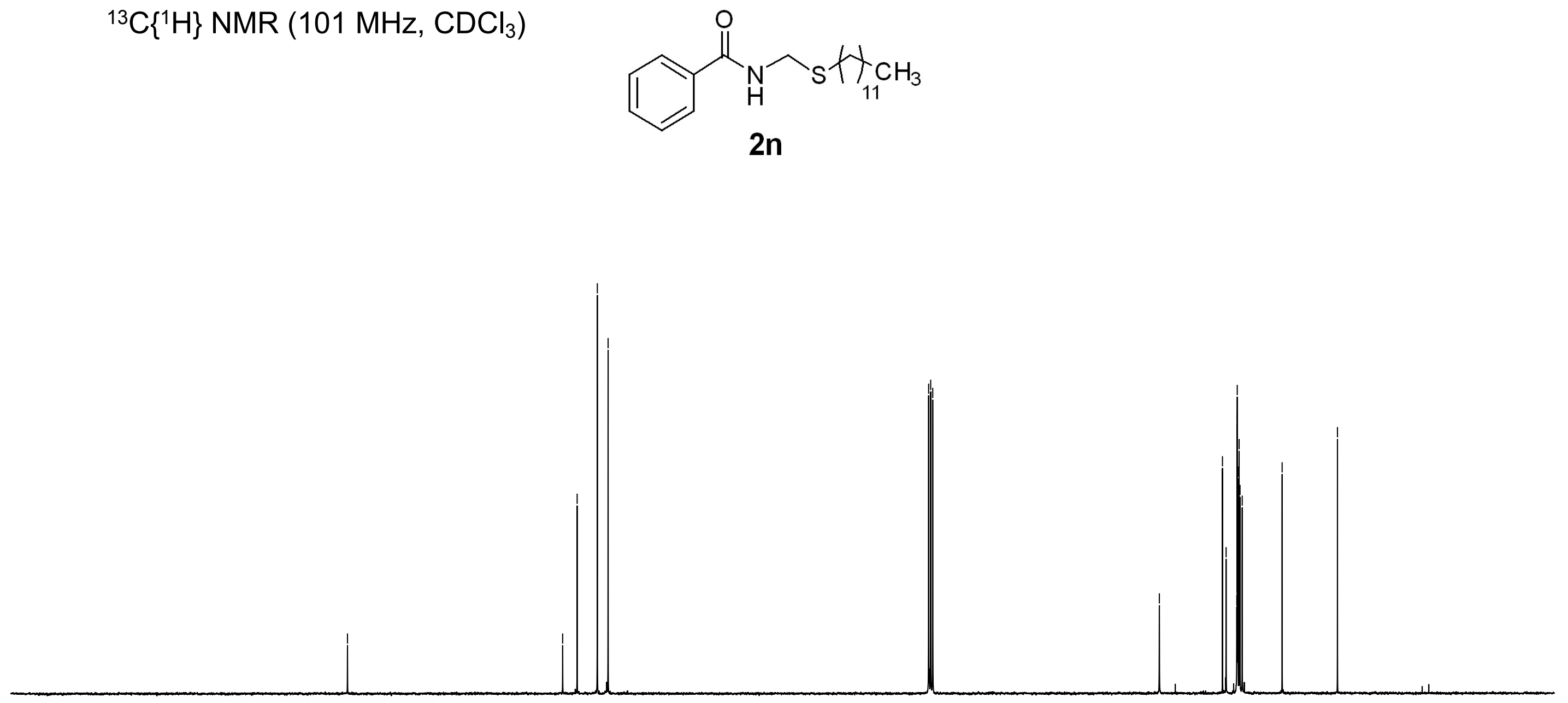

60


${ }^{1} \mathrm{H}$ NMR $\left(400 \mathrm{MHz}, \mathrm{CDCl}_{3}\right)$
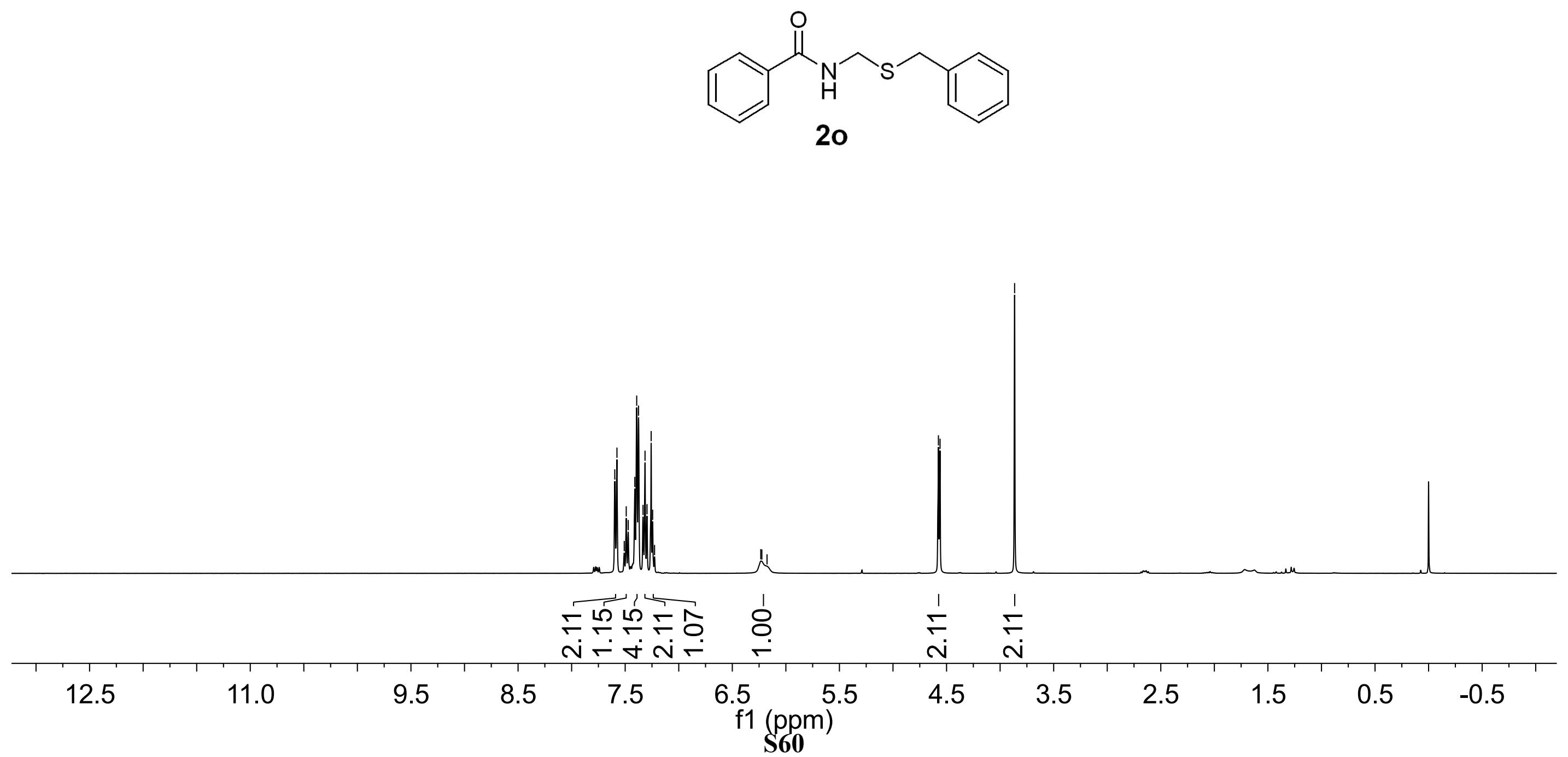
${ }^{13} \mathrm{C}\left\{{ }^{1} \mathrm{H}\right\} \operatorname{NMR}\left(101 \mathrm{MHz}, \mathrm{CDCl}_{3}\right)$<smiles>O=C(NCSCc1ccccc1)c1ccccc1</smiles>

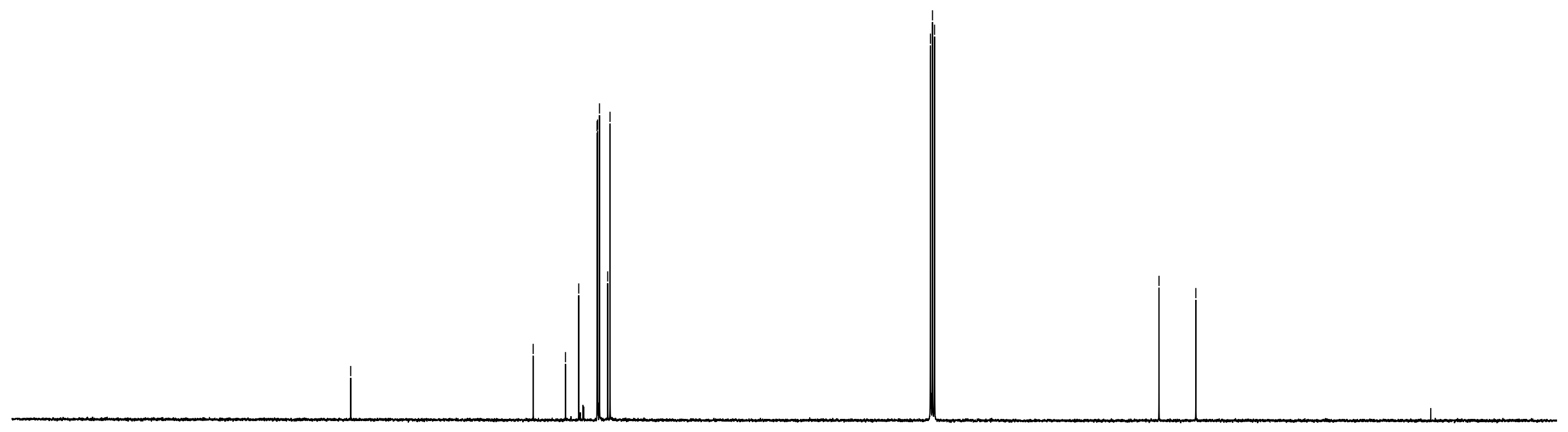

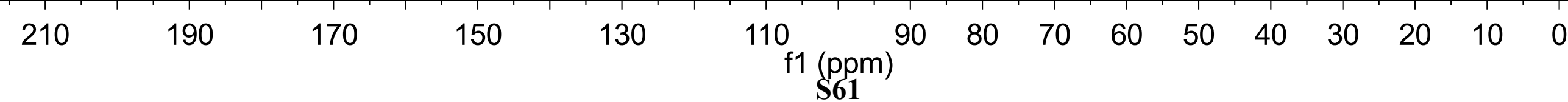


${ }^{1} \mathrm{H}$ NMR $\left(400 \mathrm{MHz}, \mathrm{CDCl}_{3}\right)$<smiles>CC(=O)NCC(=O)Sc1ccc(C)cc1</smiles>

\begin{tabular}{|c|c|c|c|c|c|c|c|c|c|c|c|c|}
\hline & & & & & 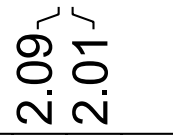 & $\stackrel{1}{\circ}$ & & ભ̊ & & 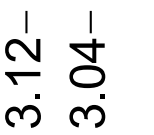 & & \\
\hline 12.0 & 11.0 & 10.0 & 9.0 & 8.0 & 7.0 & $\begin{array}{l}6.0 \\
\text { f1 (ppm) } \\
\mathbf{S 6 2}\end{array}$ & 5.0 & 4.0 & 3.0 & 2.0 & 1.0 & 0.0 \\
\hline
\end{tabular}




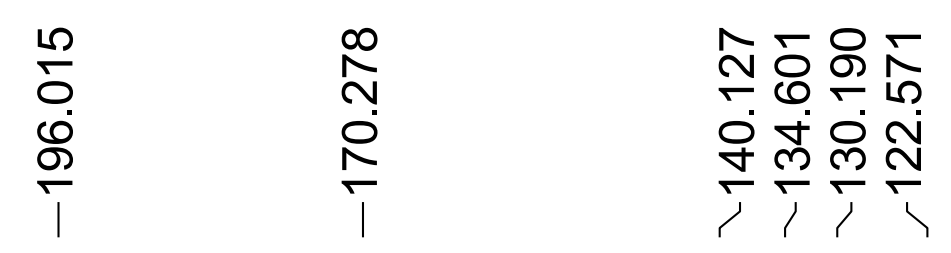

$\infty$

m。

솟

ᄋ̊

กิ่

\?

${ }^{13} \mathrm{C}\left\{{ }^{1} \mathrm{H}\right\}$ NMR $\left(101 \mathrm{MHz}, \mathrm{CDCl}_{3}\right)$<smiles>CC(=O)NCC(=O)Sc1ccc(C)cc1</smiles>

\section{$3 a$}

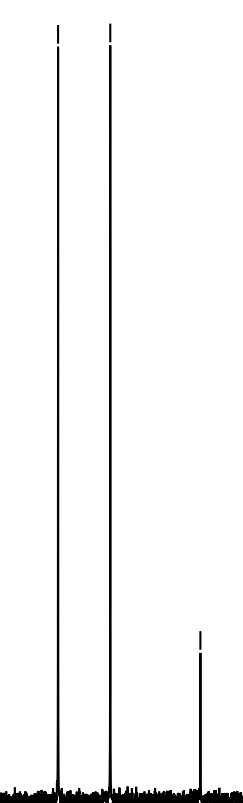

60


${ }^{1} \mathrm{H}$ NMR $\left(400 \mathrm{MHz}, \mathrm{CDCl}_{3}\right)$

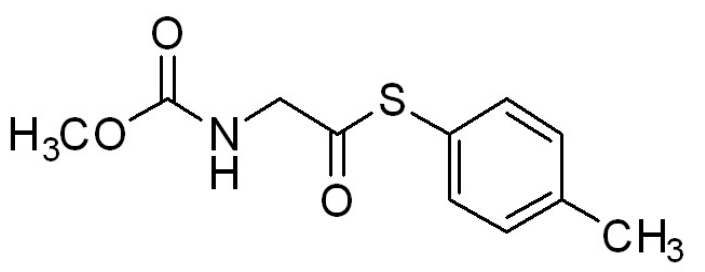

$3 b$

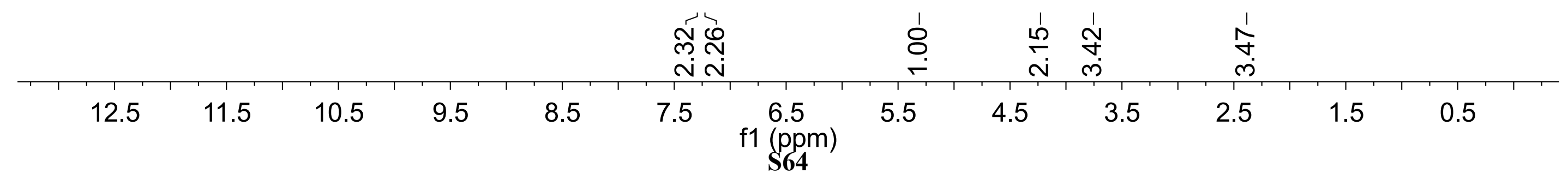


ภิ

กิ่

$\frac{\infty}{n}$

${ }^{13} \mathrm{C}\left\{{ }^{1} \mathrm{H}\right\}$ NMR $\left(101 \mathrm{MHz}, \mathrm{CDCl}_{3}\right)$<smiles>COC(=O)NCC(=O)Sc1ccc(C)cc1</smiles>

$3 b$ 
${ }^{1} \mathrm{H}$ NMR (400 MHz, $\mathrm{CDCl}_{3}$ )
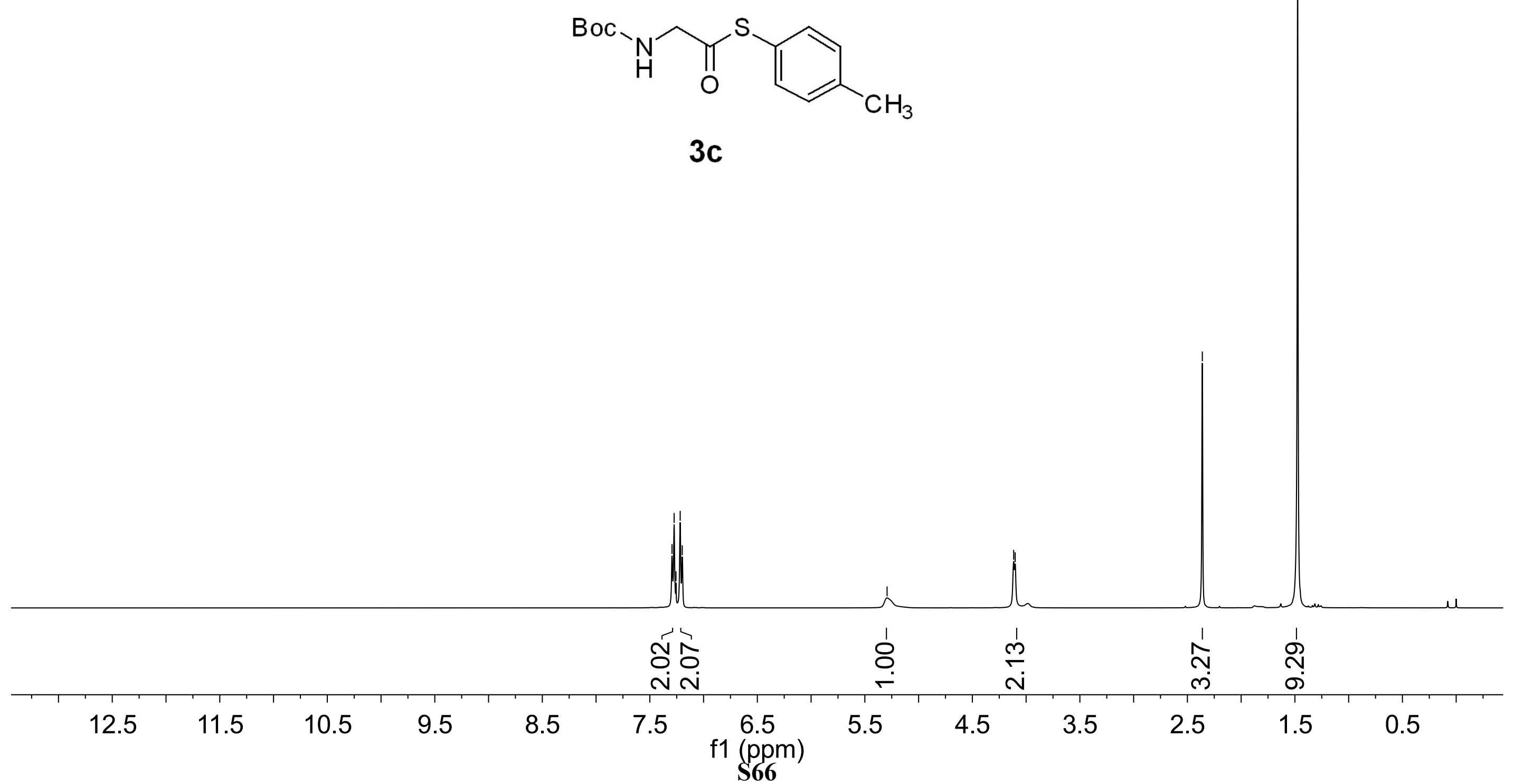
${ }^{13} \mathrm{C}\left\{{ }^{1} \mathrm{H}\right\} \operatorname{NMR}\left(101 \mathrm{MHz}, \mathrm{CDCl}_{3}\right)$

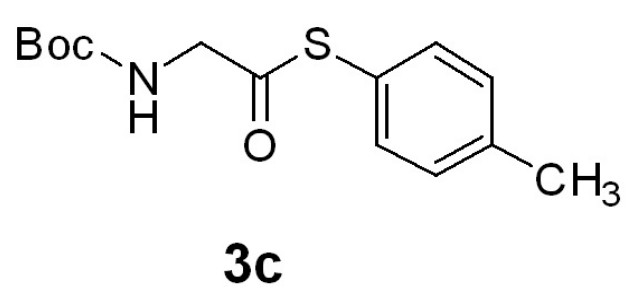

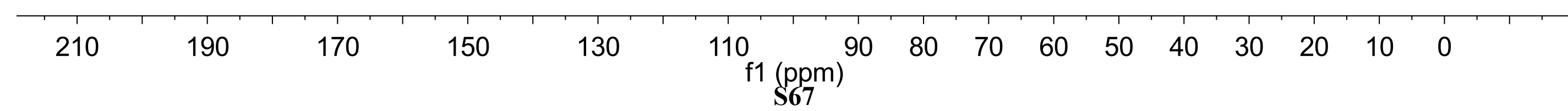


${ }^{1} \mathrm{H}$ NMR $\left(400 \mathrm{MHz}, \mathrm{CDCl}_{3}\right)$<smiles>Cc1ccc(SC(=O)CNC(=O)c2ccc3ccccc3c2)cc1</smiles>

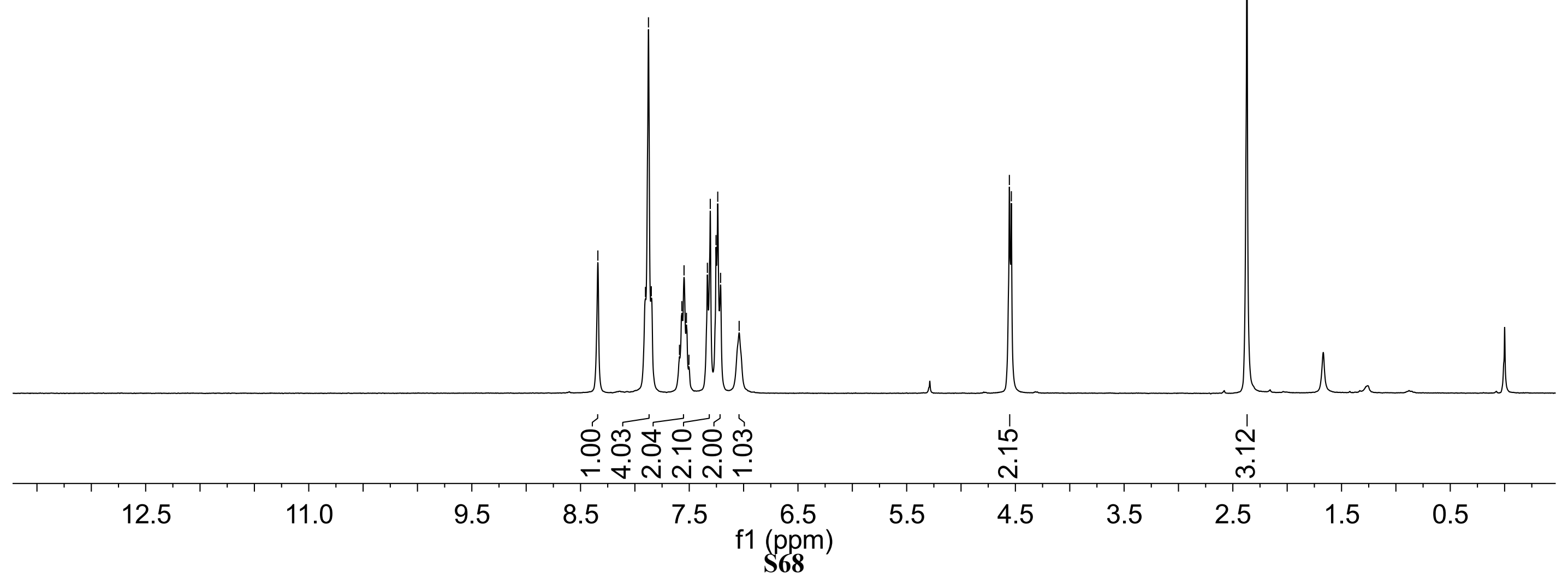


${ }^{13} \mathrm{C}\left\{{ }^{1} \mathrm{H}\right\}$ NMR $\left(101 \mathrm{MHz}, \mathrm{CDCl}_{3}\right)$<smiles>Cc1ccc(SC(=O)CNC(=O)c2ccc3ccccc3c2)cc1</smiles>

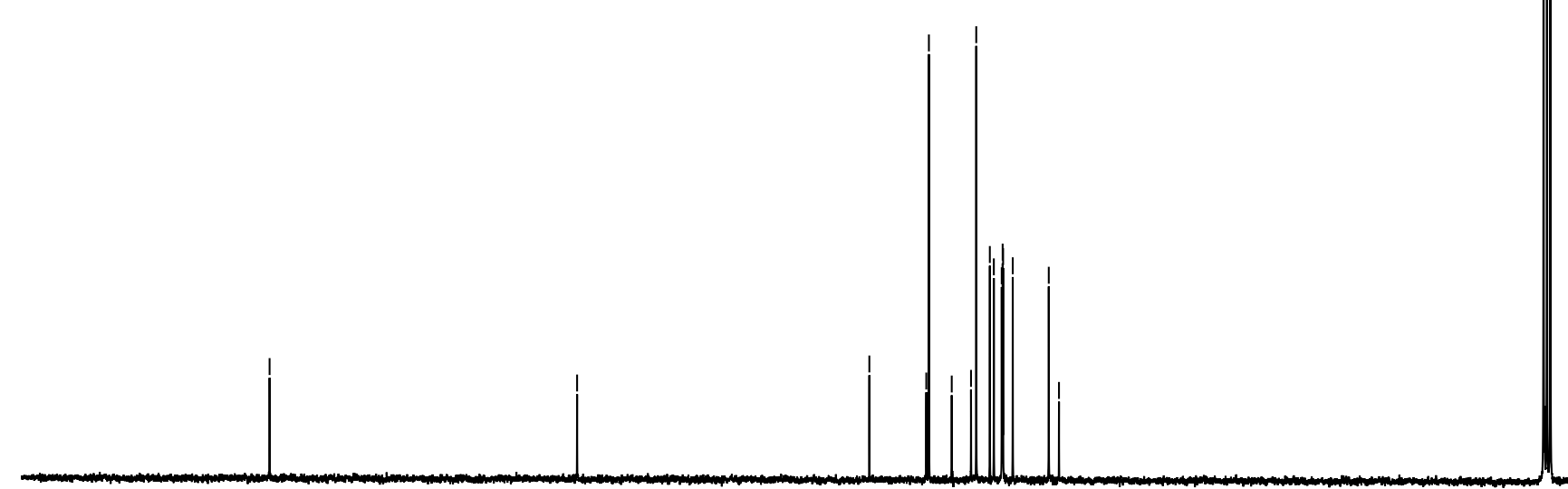


${ }^{1} \mathrm{H}$ NMR $\left(400 \mathrm{MHz}, \mathrm{CDCl}_{3}\right)$
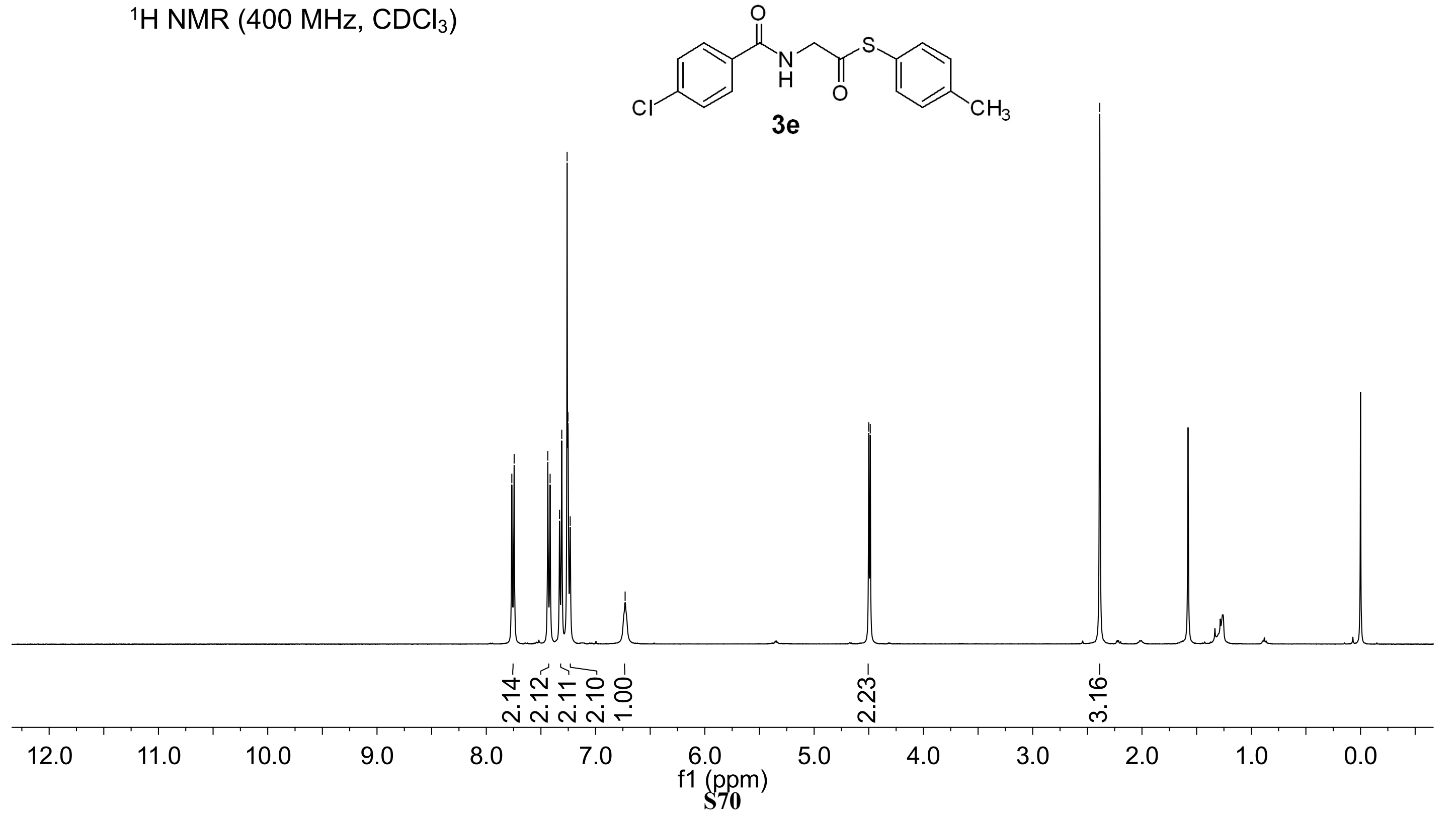
$\stackrel{m}{\sigma}$
$\stackrel{0}{\circ}$
$\frac{1}{1}$

${ }^{13} \mathrm{C}\left\{{ }^{1} \mathrm{H}\right\}$ NMR $\left(101 \mathrm{MHz}, \mathrm{CDCl}_{3}\right)$<smiles>Cc1ccc(SC(=O)CNC(=O)c2ccc(Cl)cc2)cc1</smiles>

.

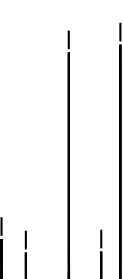


${ }^{1} \mathrm{H}$ NMR $\left(400 \mathrm{MHz}, \mathrm{CDCl}_{3}\right)$<smiles>COc1ccc(C(=O)NCC(=O)Sc2ccc(C)cc2)cc1</smiles>

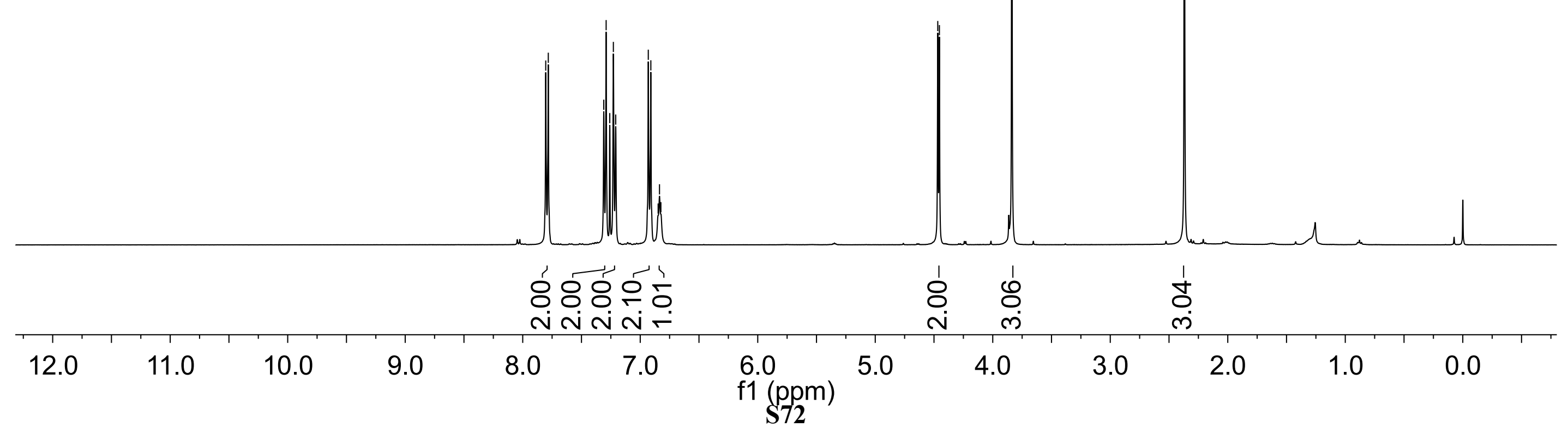


${ }^{13} \mathrm{C}\left\{{ }^{1} \mathrm{H}\right\}$ NMR (101 MHz, $\left.\mathrm{CDCl}_{3}\right)$<smiles>COc1ccc(C(=O)NCC(=O)Sc2ccc(C)cc2)cc1</smiles>

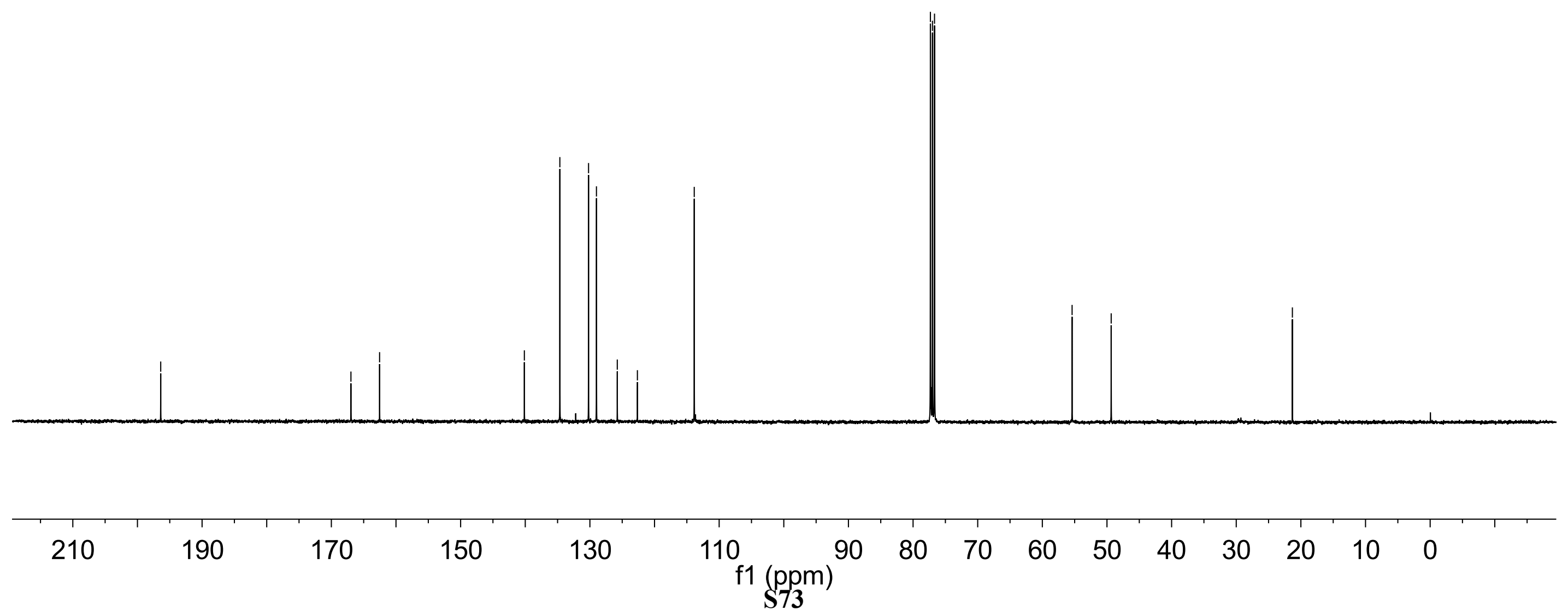


${ }^{1} \mathrm{H}$ NMR $\left(400 \mathrm{MHz}, \mathrm{CDCl}_{3}\right)$<smiles>Cc1ccc(SC(=O)CNC(=O)Cc2ccccc2)cc1</smiles>

\begin{tabular}{|c|c|c|c|c|c|c|c|c|c|c|c|c|}
\hline & & & & & 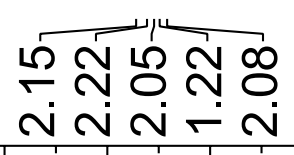 & o & & $\stackrel{1}{\infty}$ & $\frac{1}{\infty}$ & $\frac{1}{\pi}$ & & \\
\hline 12.5 & 11.5 & 10.5 & 9.5 & 8.5 & $\mathrm{f1}$ & & 5.5 & 4.5 & 3.5 & 2.5 & 1.5 & 0.5 \\
\hline
\end{tabular}



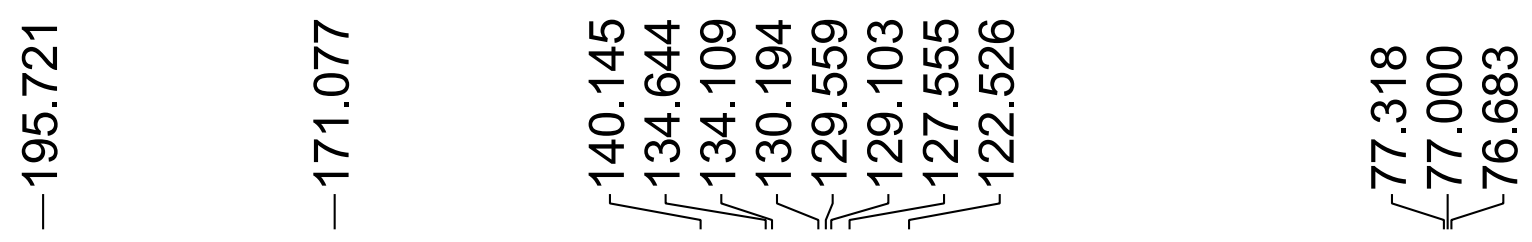

\begin{tabular}{ll}
5 & 5 \\
$\infty$ & $\infty$ \\
$\infty$ & $n$ \\
\multirow{1}{*}{} & \multirow{1}{*}{}
\end{tabular}

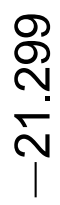

${ }^{13} \mathrm{C}\left\{{ }^{1} \mathrm{H}\right\} \operatorname{NMR}\left(101 \mathrm{MHz}, \mathrm{CDCl}_{3}\right)$
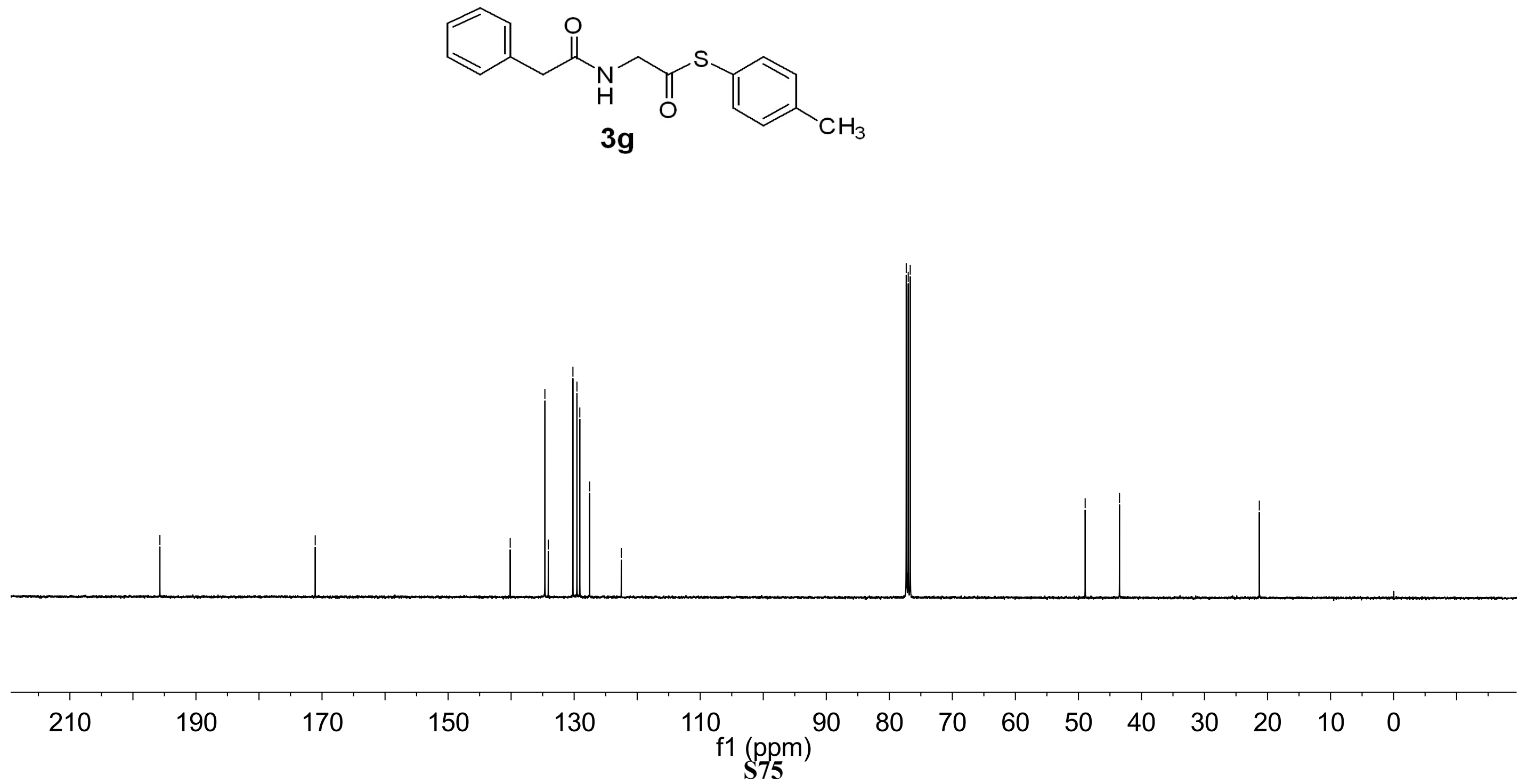
${ }^{1} \mathrm{H}$ NMR $\left(400 \mathrm{MHz}, \mathrm{CDCl}_{3}\right)$<smiles>Cc1ccc(SC(=O)CN2C(=O)c3ccccc3C2=O)cc1</smiles>

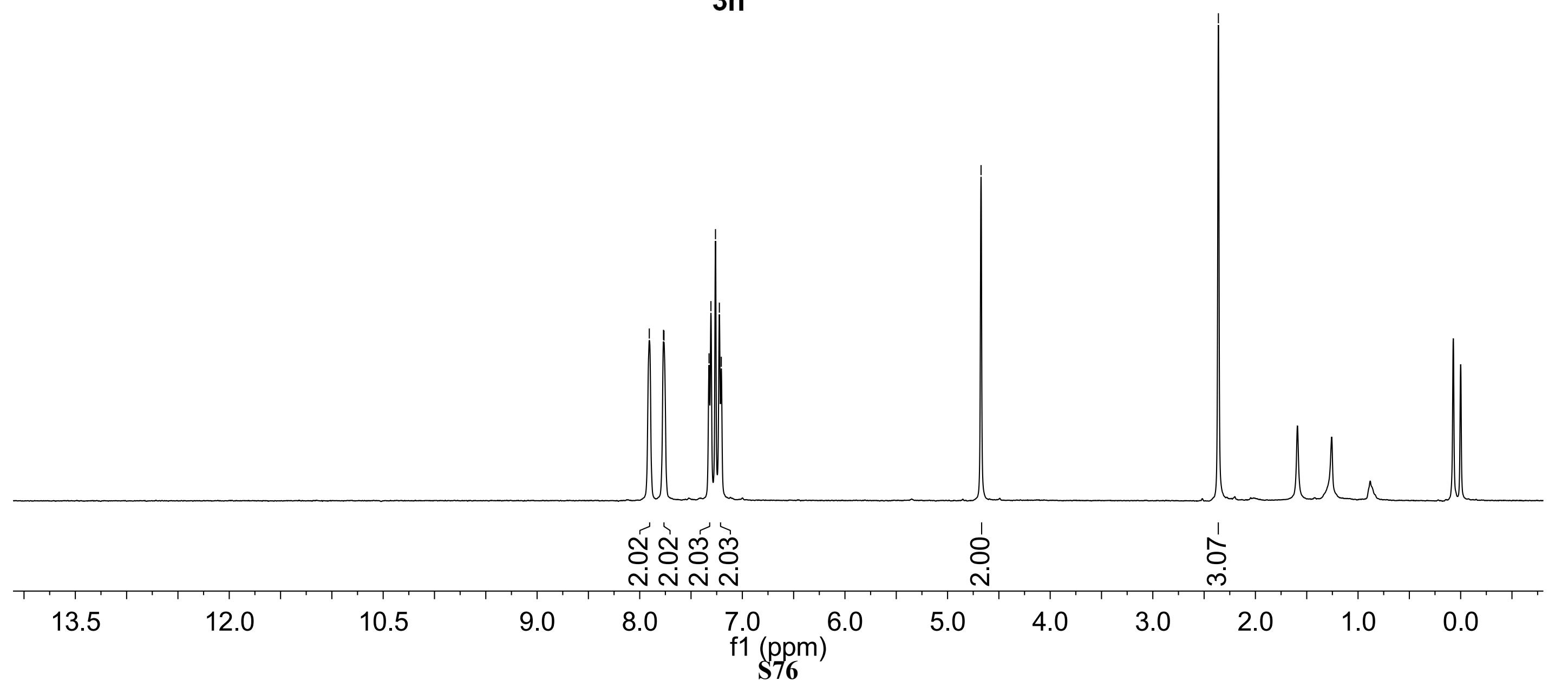



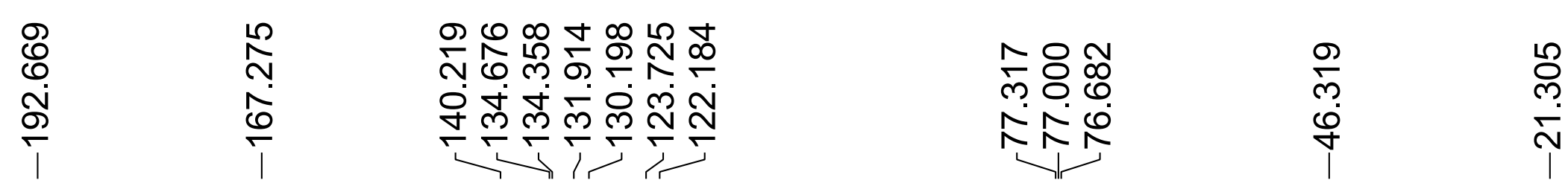

${ }^{13} \mathrm{C}\left\{{ }^{1} \mathrm{H}\right\} \operatorname{NMR}\left(101 \mathrm{MHz}, \mathrm{CDCl}_{3}\right)$
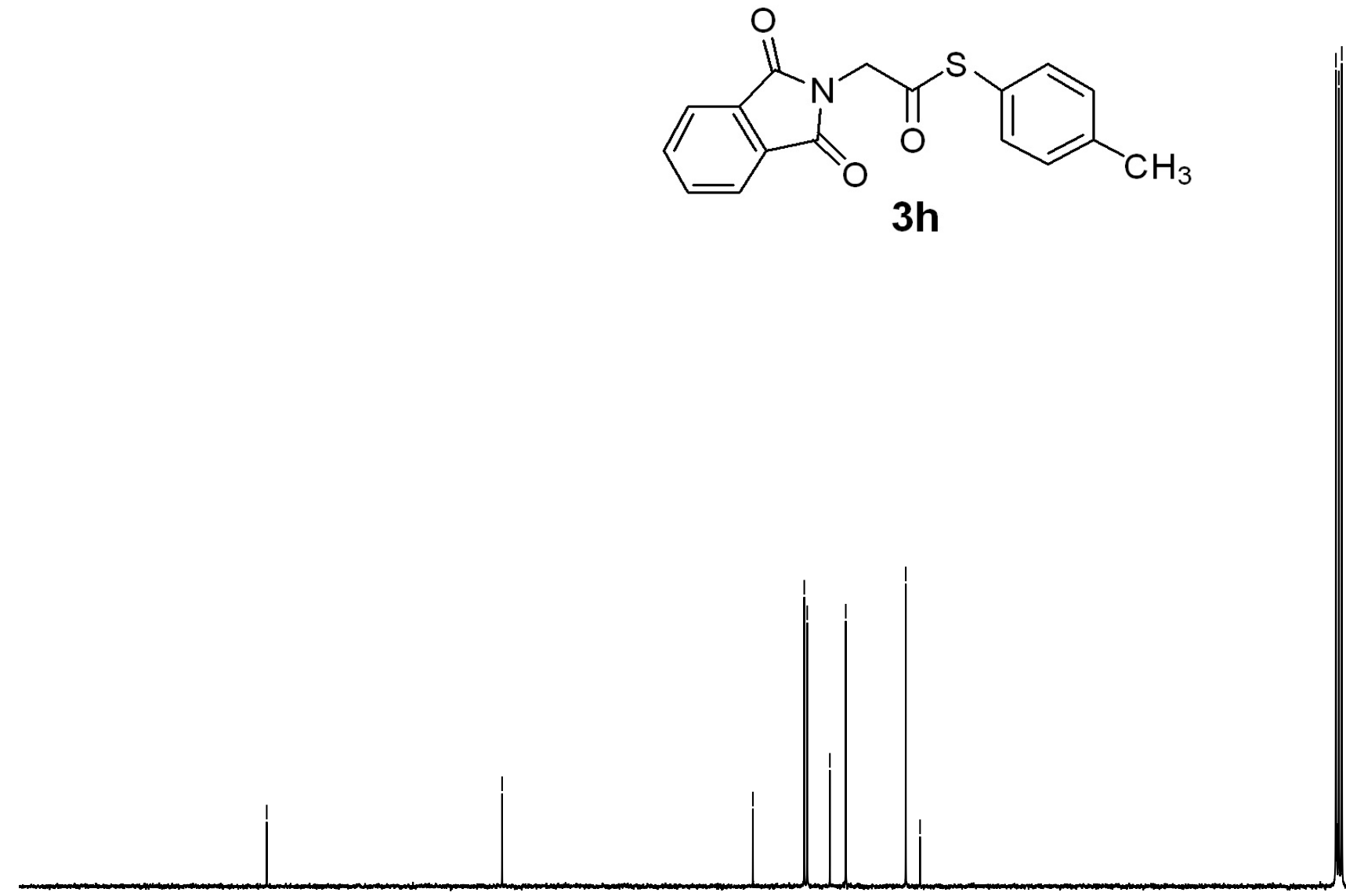

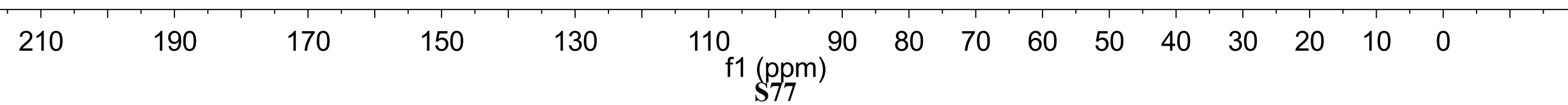


${ }^{1} \mathrm{H}$ NMR $\left(400 \mathrm{MHz}, \mathrm{CDCl}_{3}\right)$
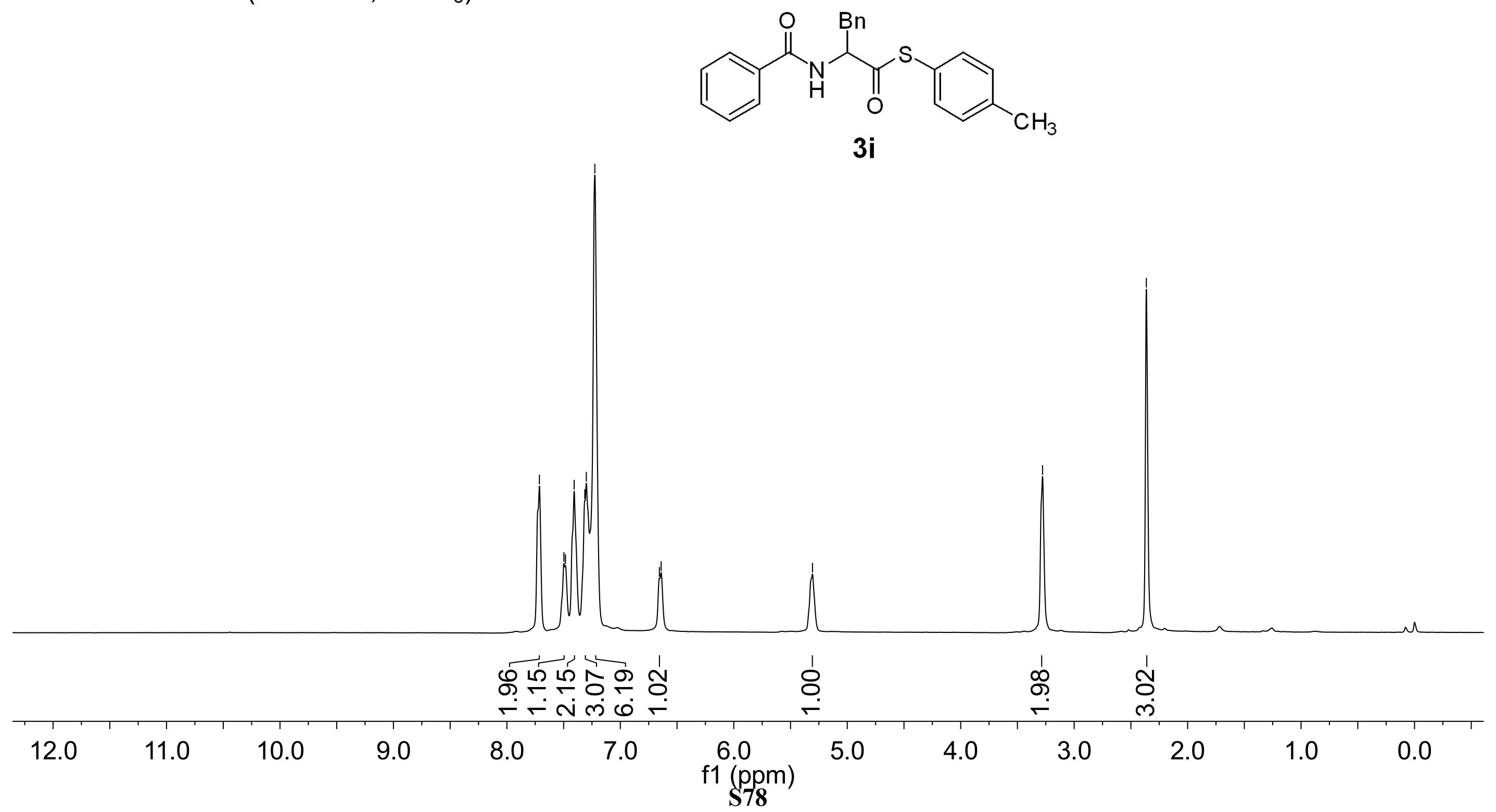


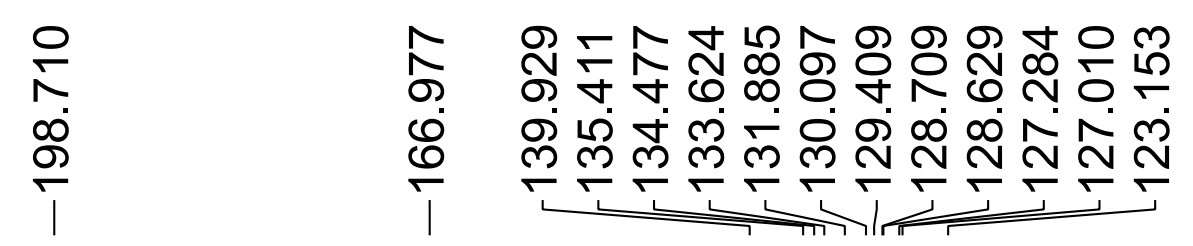

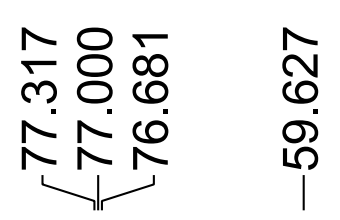

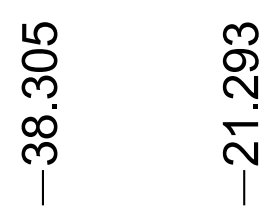
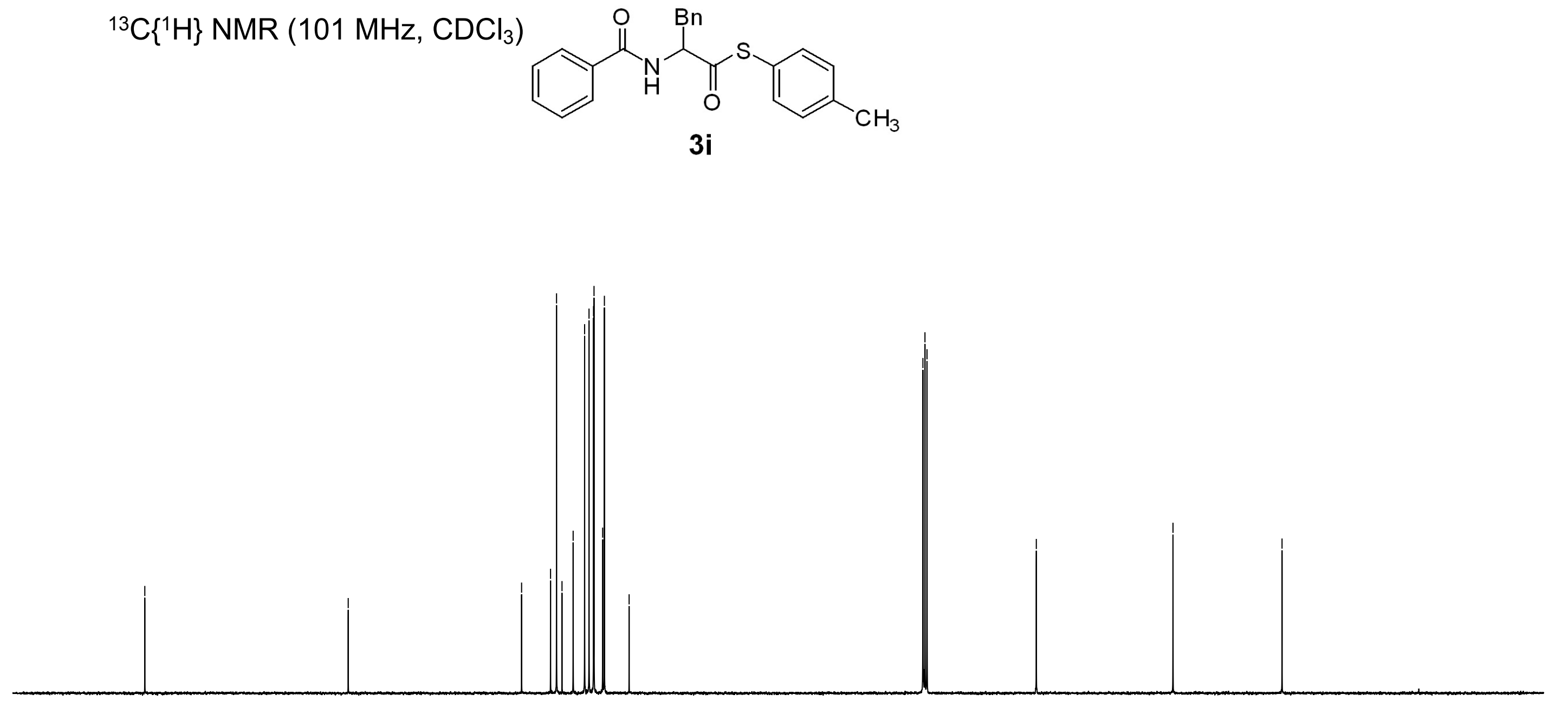

\begin{tabular}{|c|c|c|c|c|c|c|c|c|c|c|c|c|c|c|}
\hline 210 & 190 & 170 & 150 & 130 & $\begin{array}{c}110 \\
\mathrm{f1} \text { (ppm) } \\
\mathbf{S 7 9}\end{array}$ & 80 & 70 & 60 & 50 & 40 & 30 & 20 & 10 & 0 \\
\hline
\end{tabular}


${ }^{1} \mathrm{H}$ NMR $\left(400 \mathrm{MHz}, \mathrm{CDCl}_{3}\right)$
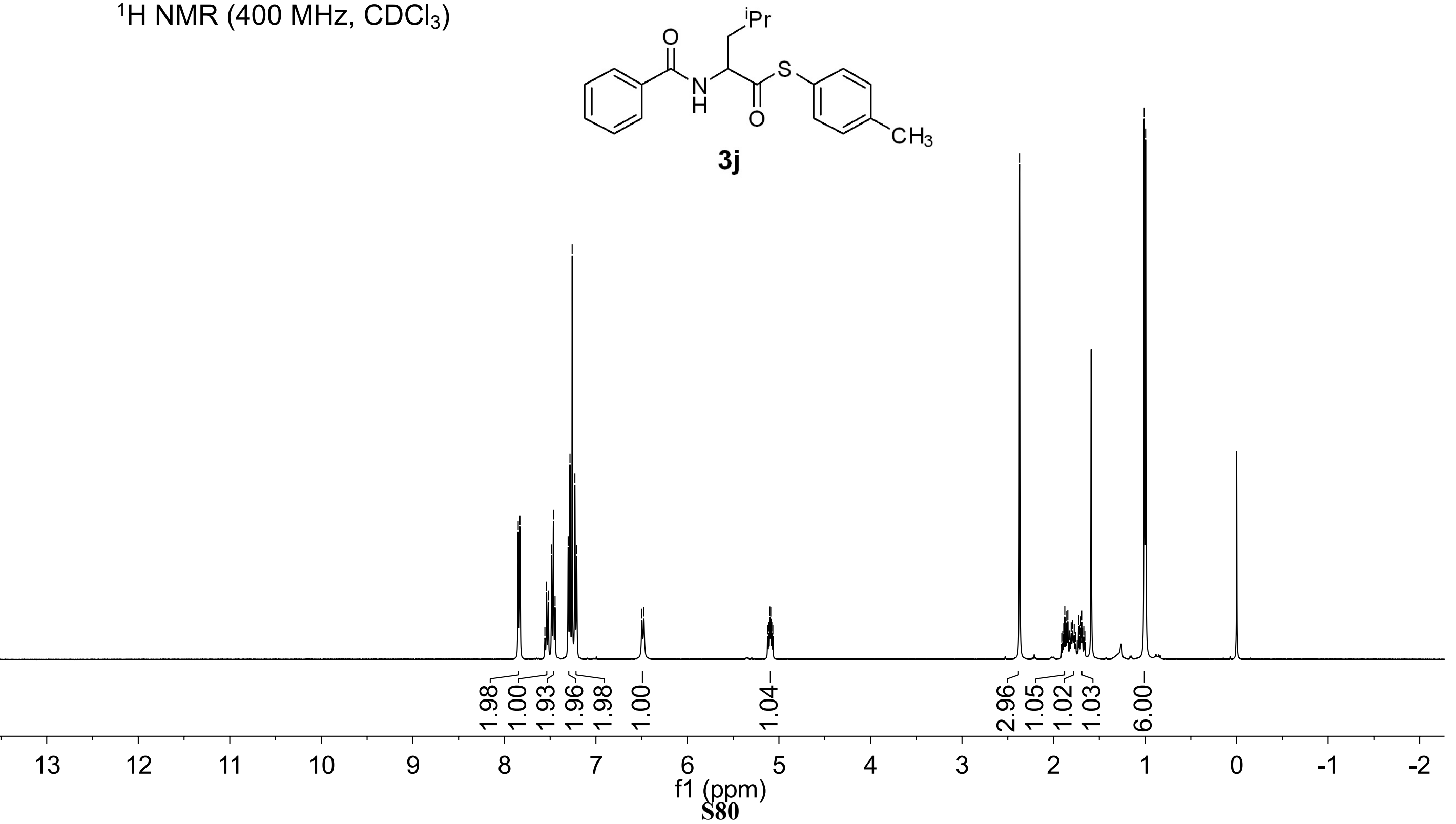
${ }^{13} \mathrm{C}\left\{{ }^{1} \mathrm{H}\right\}$ NMR (101 MHz, $\left.\mathrm{CDCl}_{3}\right)$<smiles>Cc1ccc(SC(=O)C(CC(C)C)NC(=O)c2ccccc2)cc1</smiles>

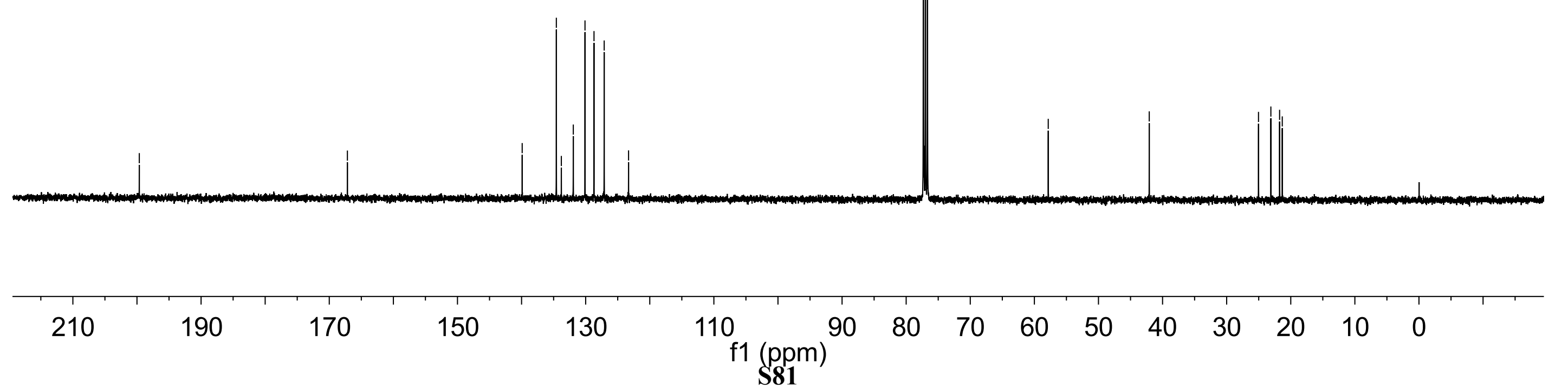


${ }^{1} \mathrm{H}$ NMR $\left(400 \mathrm{MHz}, \mathrm{CDCl}_{3}\right)$
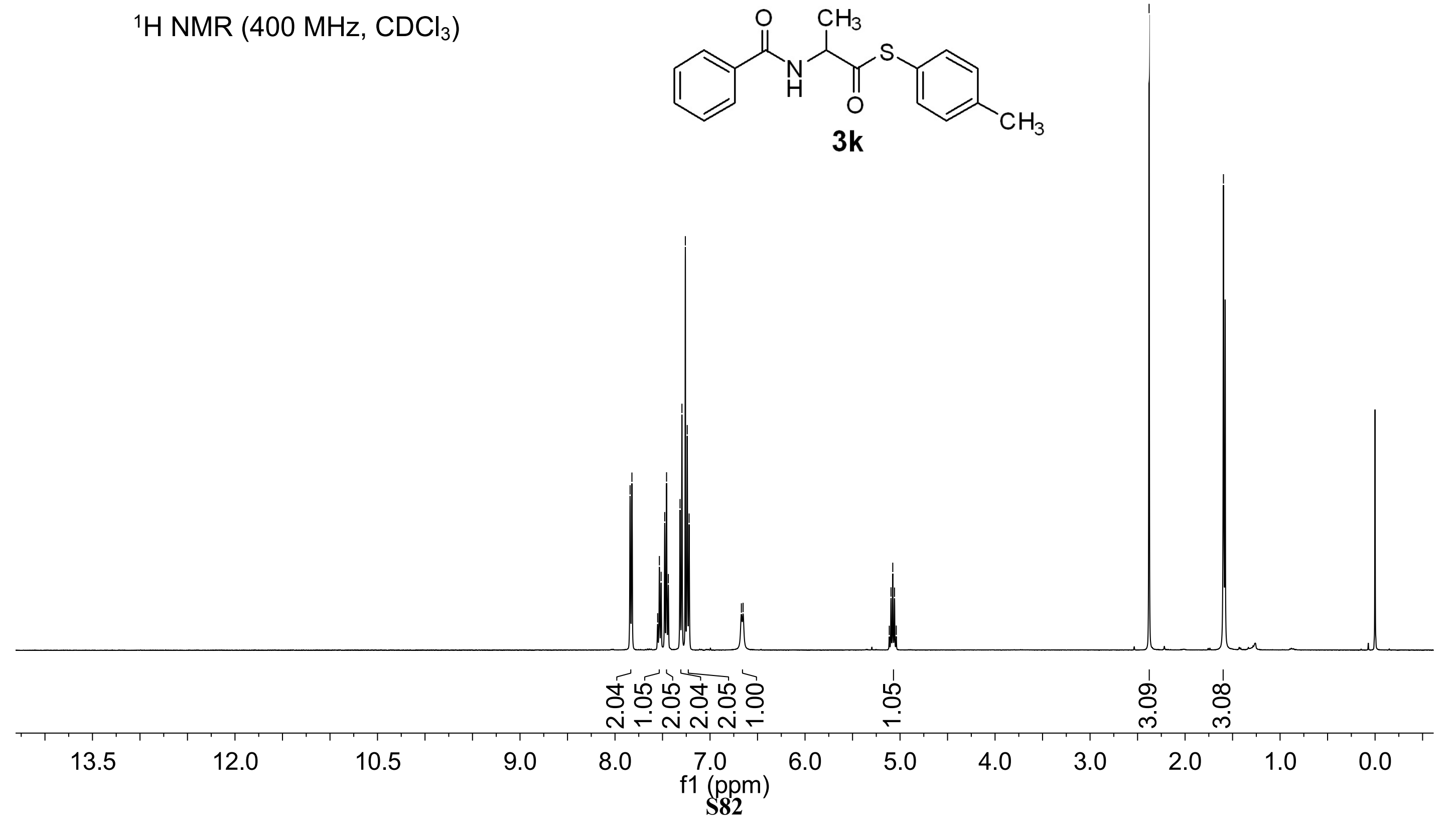
${ }^{13} \mathrm{C}\left\{{ }^{1} \mathrm{H}\right\}$ NMR $\left(101 \mathrm{MHz}, \mathrm{CDCl}_{3}\right)$<smiles>Cc1ccc(SC(=O)C(C)NC(=O)c2ccccc2)cc1</smiles>
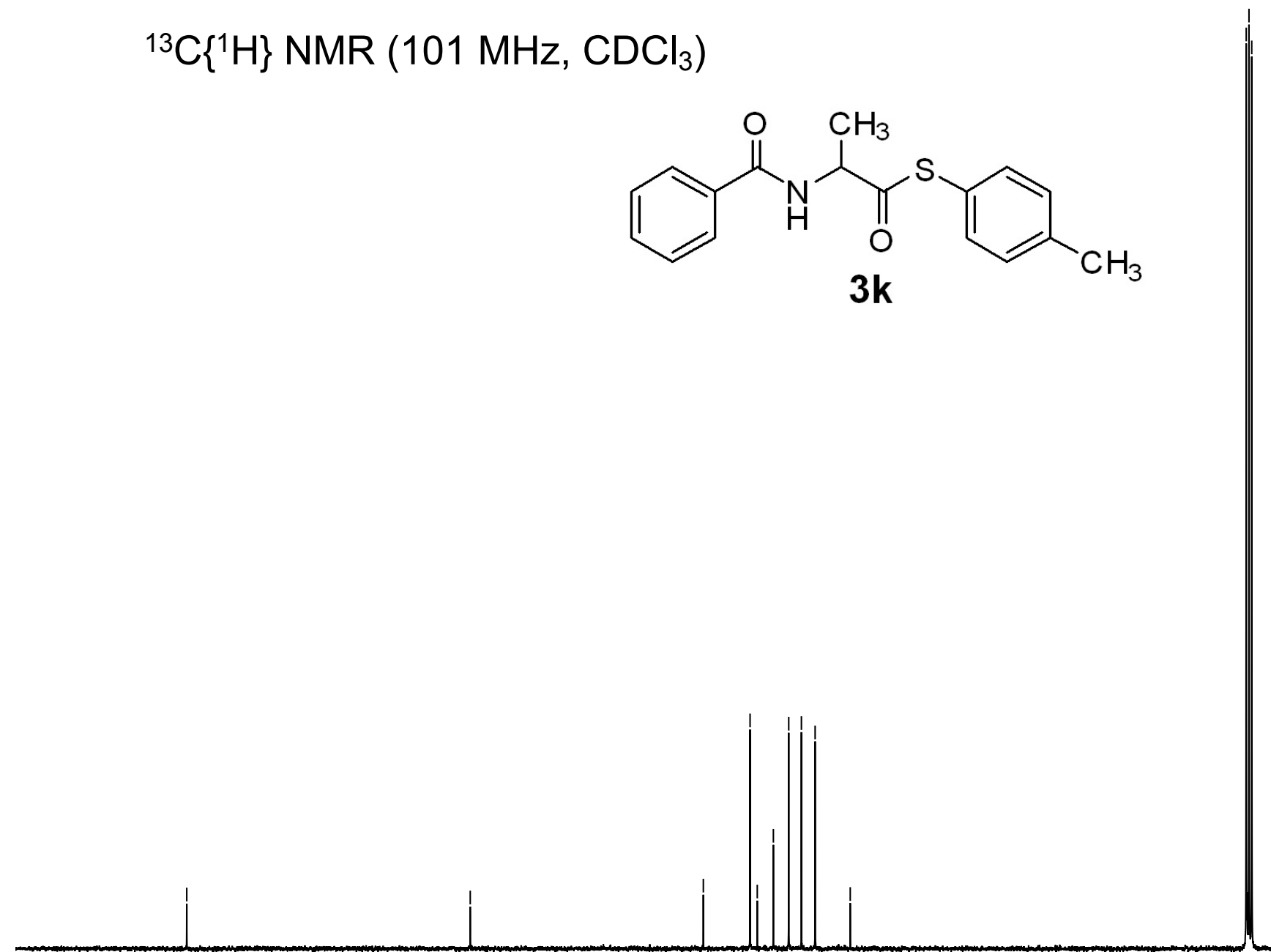

\begin{tabular}{llllllllllllllllll}
\hline 210 & 190 & 170 & 150 & 130 & $\begin{array}{c}110 \\
\mathrm{f1}(\mathrm{ppm})\end{array}$ & 90 & 80 & 70 & 60 & 50 & 40 & 30 & 20 & 10 & 0 &
\end{tabular}


${ }^{1} \mathrm{H}$ NMR $\left(400 \mathrm{MHz}, \mathrm{CDCl}_{3}\right)$<smiles>CC(=O)NC(CC(C)C)C(=O)Sc1ccc(C)cc1</smiles>

31

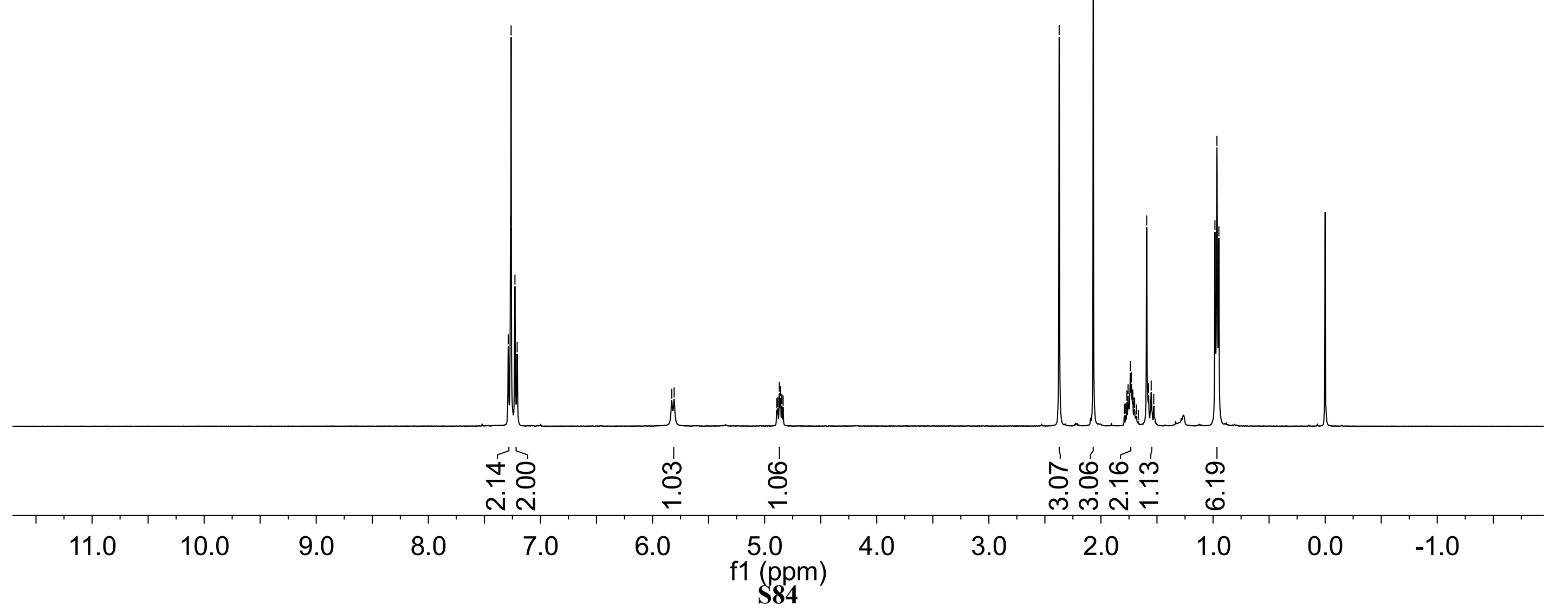


ำ
ஓं
$\frac{\operatorname{l}}{1}$

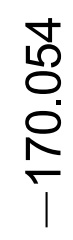

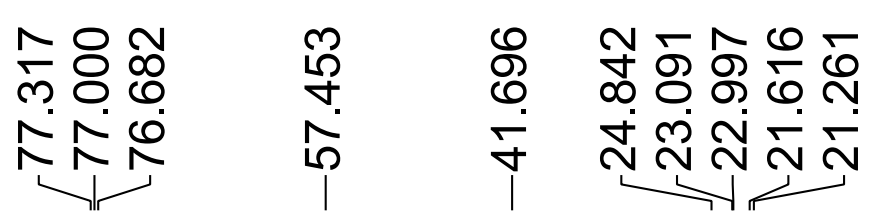

${ }^{13} \mathrm{C}\left\{{ }^{1} \mathrm{H}\right\}$ NMR $\left(101 \mathrm{MHz}, \mathrm{CDCl}_{3}\right)$
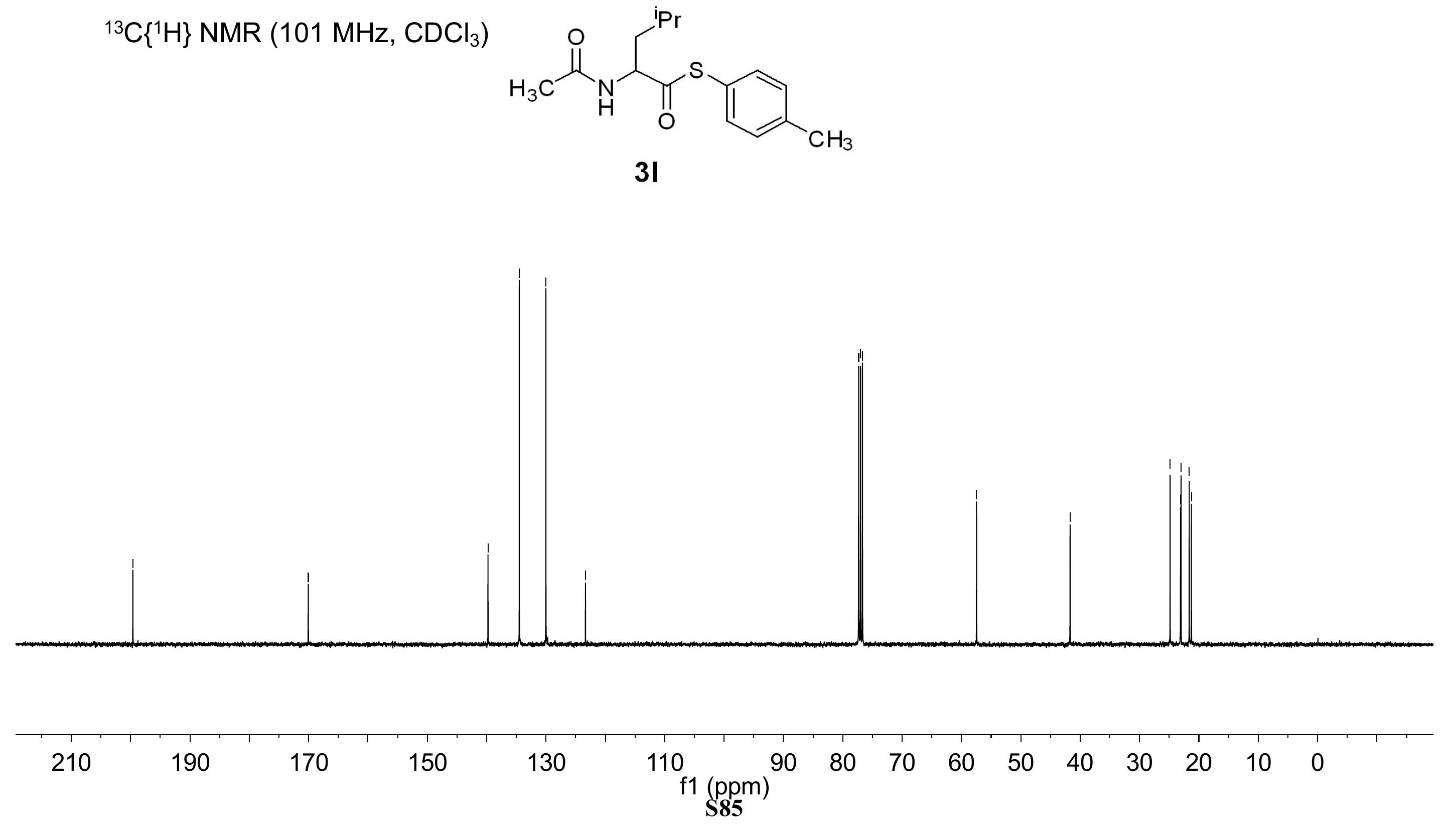
${ }^{1} \mathrm{H}$ NMR $\left(400 \mathrm{MHz} \mathrm{CDCl}_{3}\right)$
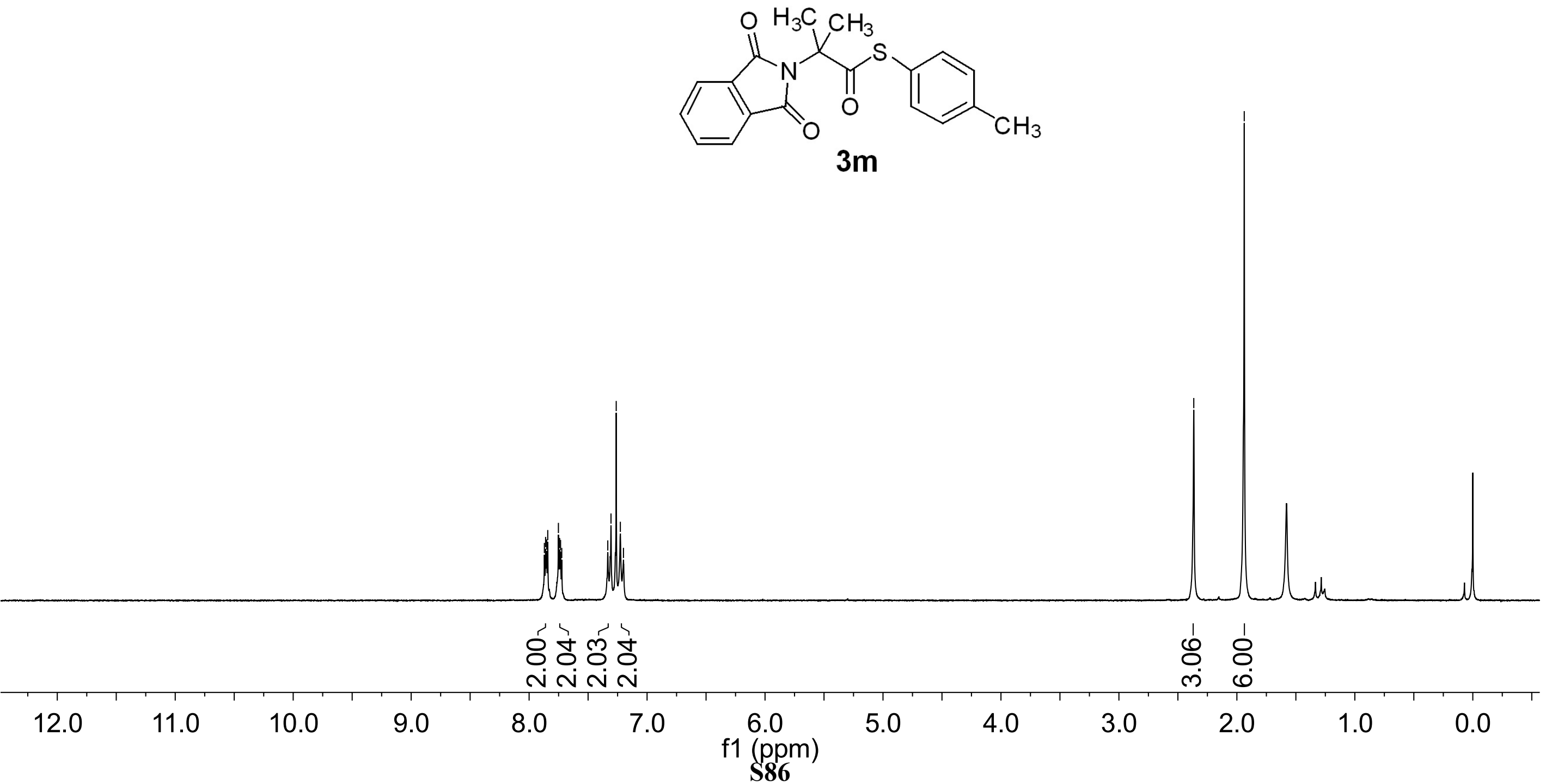


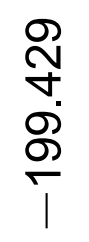

${ }^{13} \mathrm{C}\left\{{ }^{1} \mathrm{H}\right\}$ NMR $\left(101 \mathrm{MHz}, \mathrm{CDCl}_{3}\right)$<smiles>Cc1ccc(SC(=O)C(C)(C)N2C(=O)c3ccccc3C2=O)cc1</smiles>

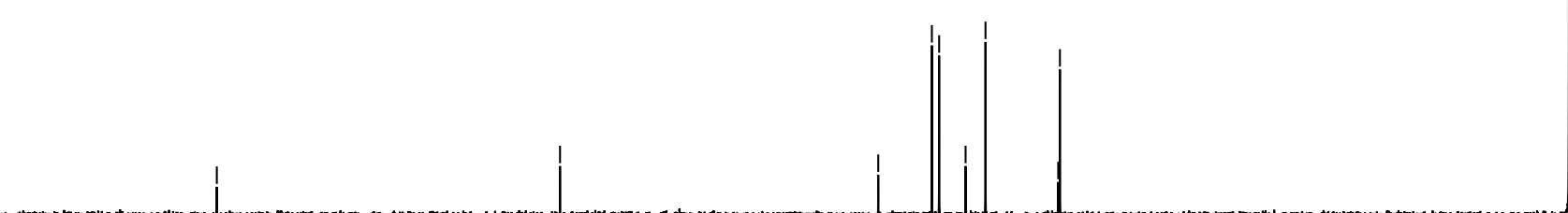

\begin{tabular}{|c|c|c|c|c|c|c|c|c|c|c|c|c|c|c|c|}
\hline 210 & 190 & 170 & 150 & 130 & ${ }_{\mathrm{f} 1}^{110}$ & $\mathrm{~m})^{90}$ & 80 & 70 & 60 & 50 & 40 & 30 & 20 & 10 & 0 \\
\hline
\end{tabular}


${ }^{1} \mathrm{H}$ NMR $\left(400 \mathrm{MHz}, \mathrm{CDCl}_{3}\right)$
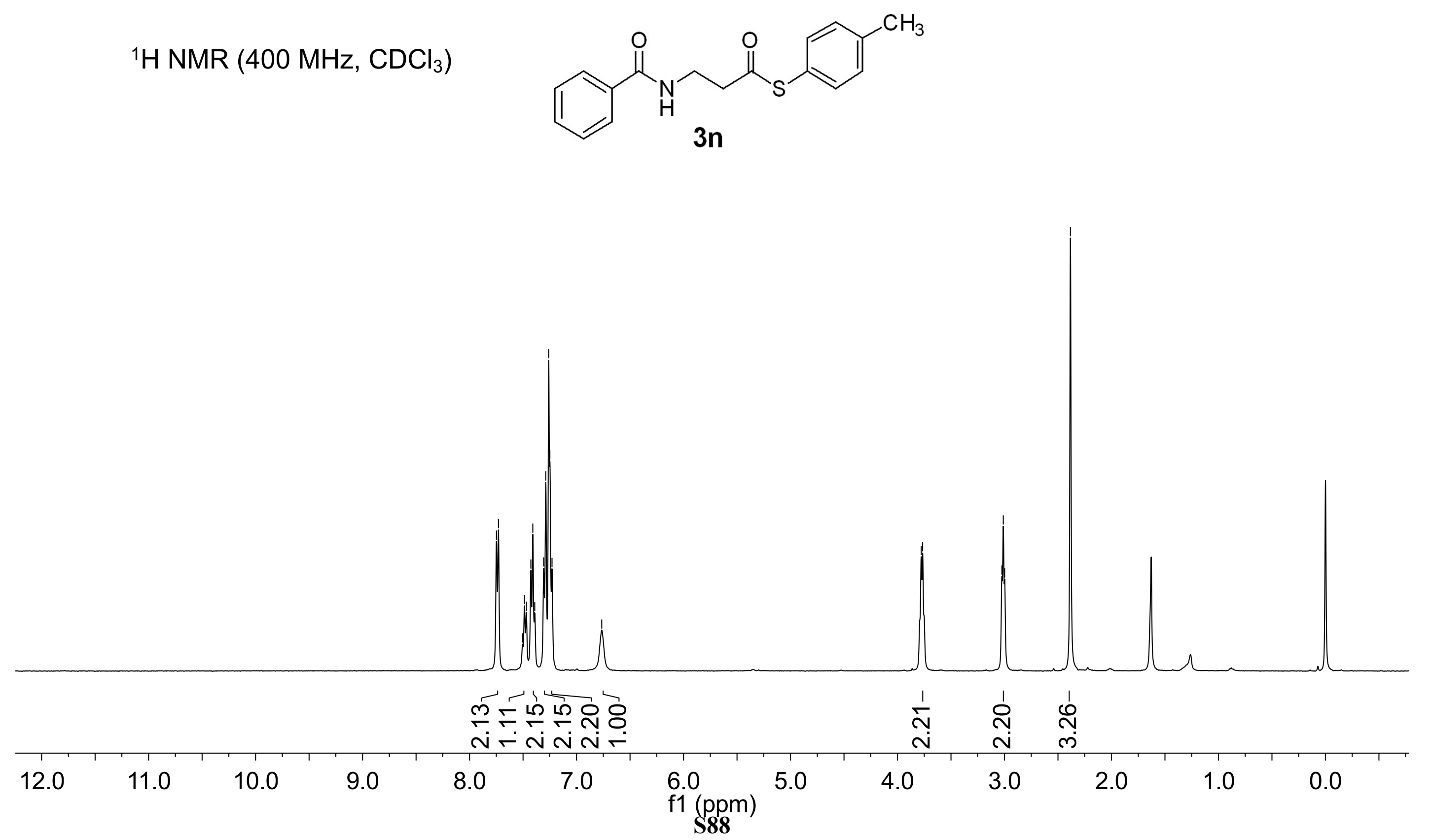
\begin{tabular}{l}
10 \\
$\infty$ \\
1 \\
$\infty$ \\
$\infty$ \\
\hdashline \\
\hline
\end{tabular}

${ }^{13} \mathrm{C}\left\{{ }^{1} \mathrm{H}\right\} \operatorname{NMR}\left(101 \mathrm{MHz}, \mathrm{CDCl}_{3}\right)$<smiles>Cc1ccc(SC(=O)CCNC(=O)c2ccccc2)cc1</smiles>
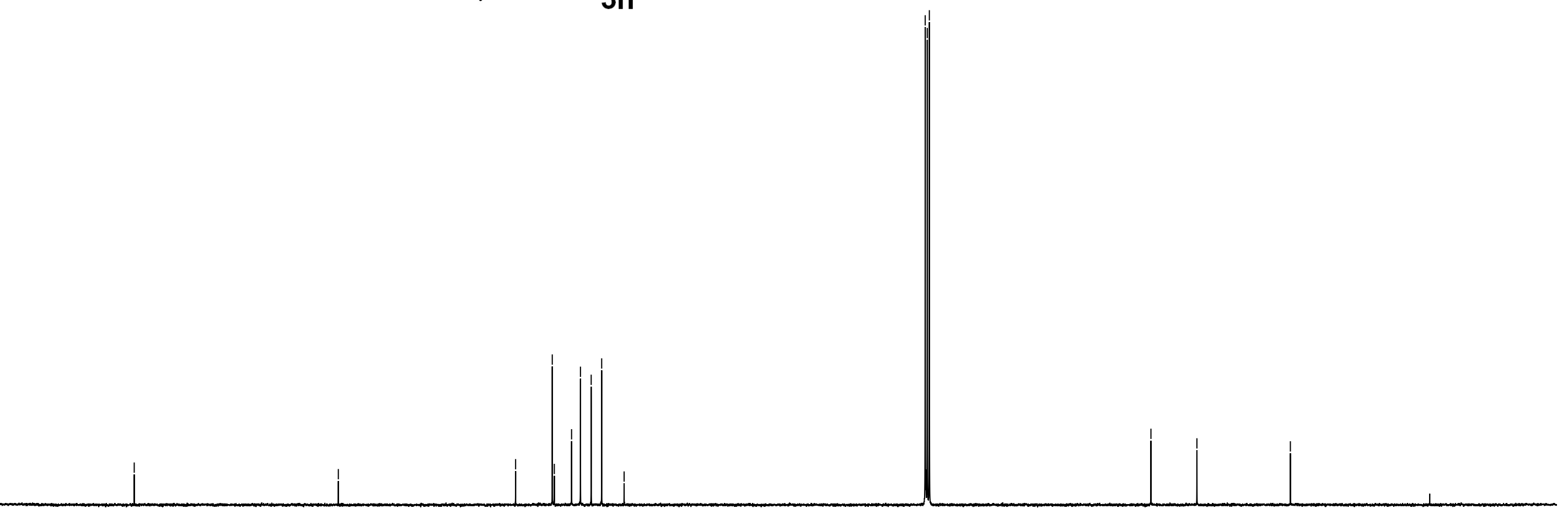

\begin{tabular}{|c|c|c|c|c|c|c|c|c|c|c|c|c|c|c|c|}
\hline 210 & 190 & 170 & 150 & 130 & $\begin{array}{r}110 \\
\mathrm{f} 1\end{array}$ & & 80 & 70 & 60 & 50 & 40 & 30 & 20 & 10 & 0 \\
\hline
\end{tabular}


${ }^{1} \mathrm{H}$ NMR $\left(400 \mathrm{MHz}, \mathrm{CDCl}_{3}\right)$<smiles>CC(=O)NCSc1ccc(C)cc1</smiles>

$4 a$

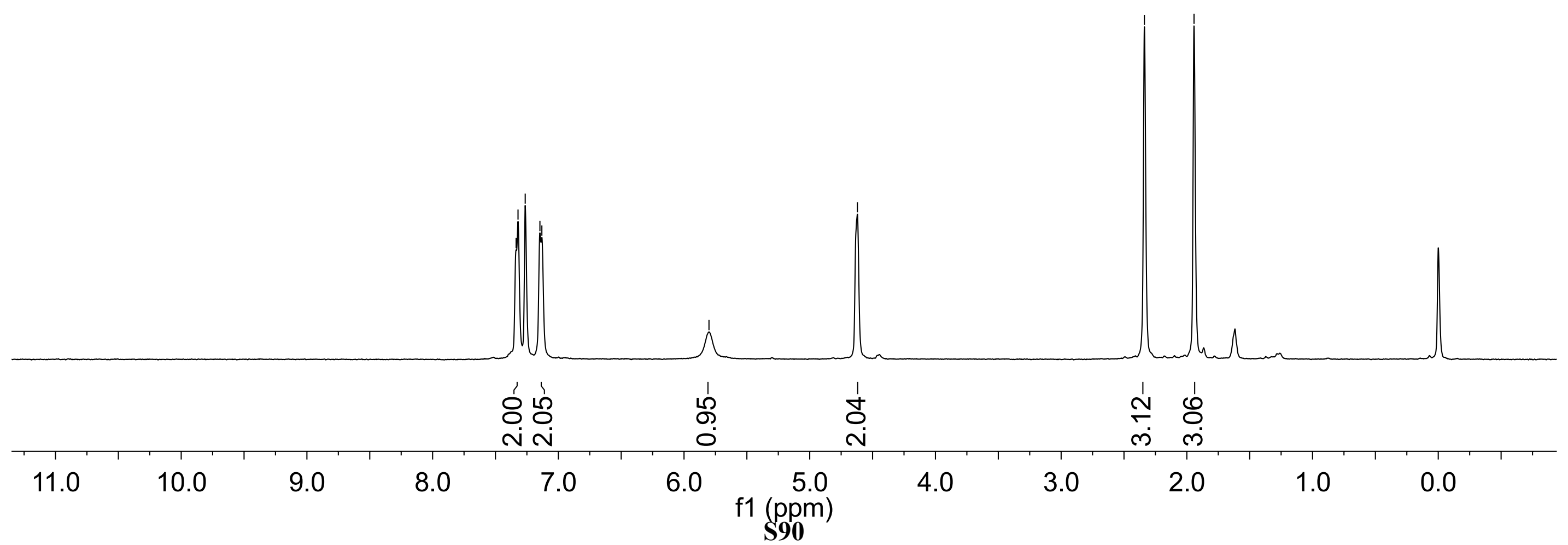


${ }^{13} \mathrm{C}\left\{{ }^{1} \mathrm{H}\right\}$ NMR $\left(101 \mathrm{MHz}, \mathrm{CDCl}_{3}\right)$<smiles>CC(=O)NCSc1ccc(C)cc1</smiles>

$4 a$

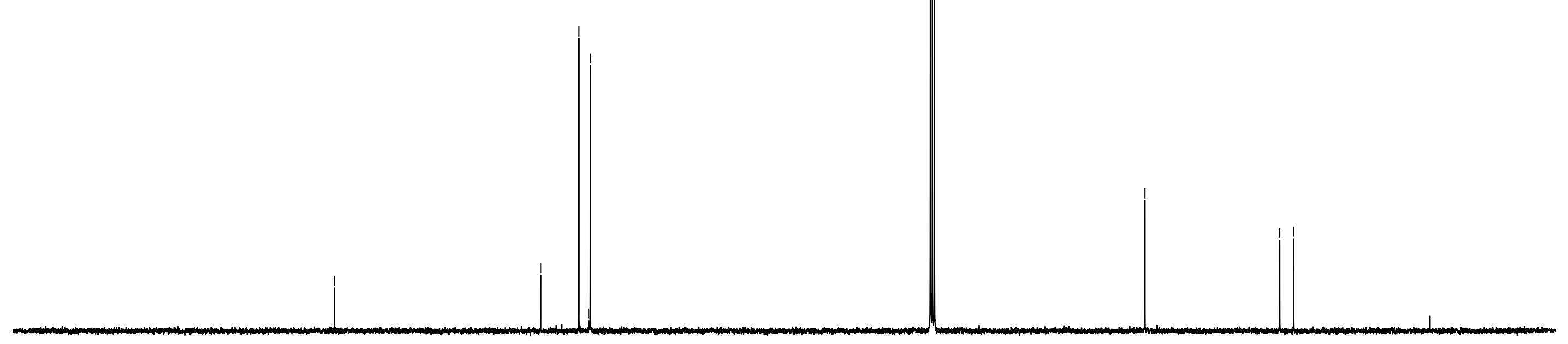

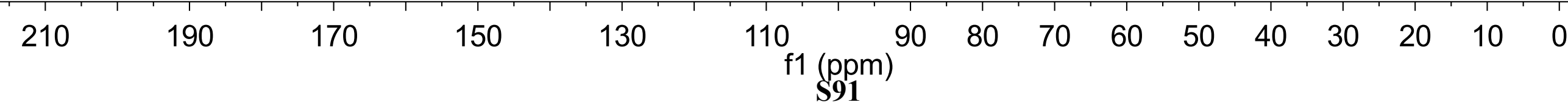


${ }^{1} \mathrm{H}$ NMR $\left(400 \mathrm{MHz}, \mathrm{CDCl}_{3}\right)$
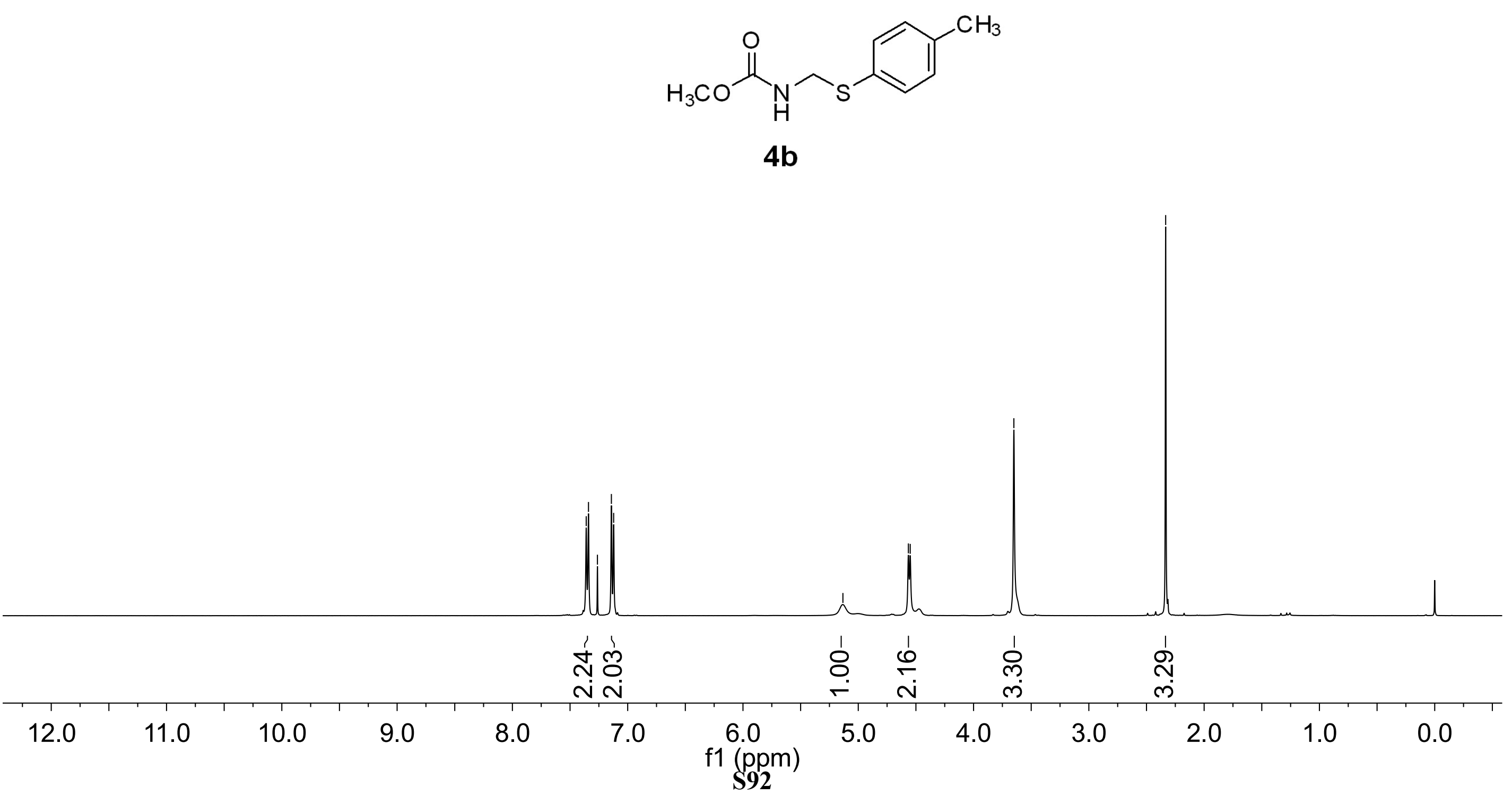
${ }^{13} \mathrm{C}\left\{{ }^{1} \mathrm{H}\right\}$ NMR $\left(101 \mathrm{MHz}, \mathrm{CDCl}_{3}\right)$<smiles>COC(=O)NCSc1ccc(C)cc1</smiles>

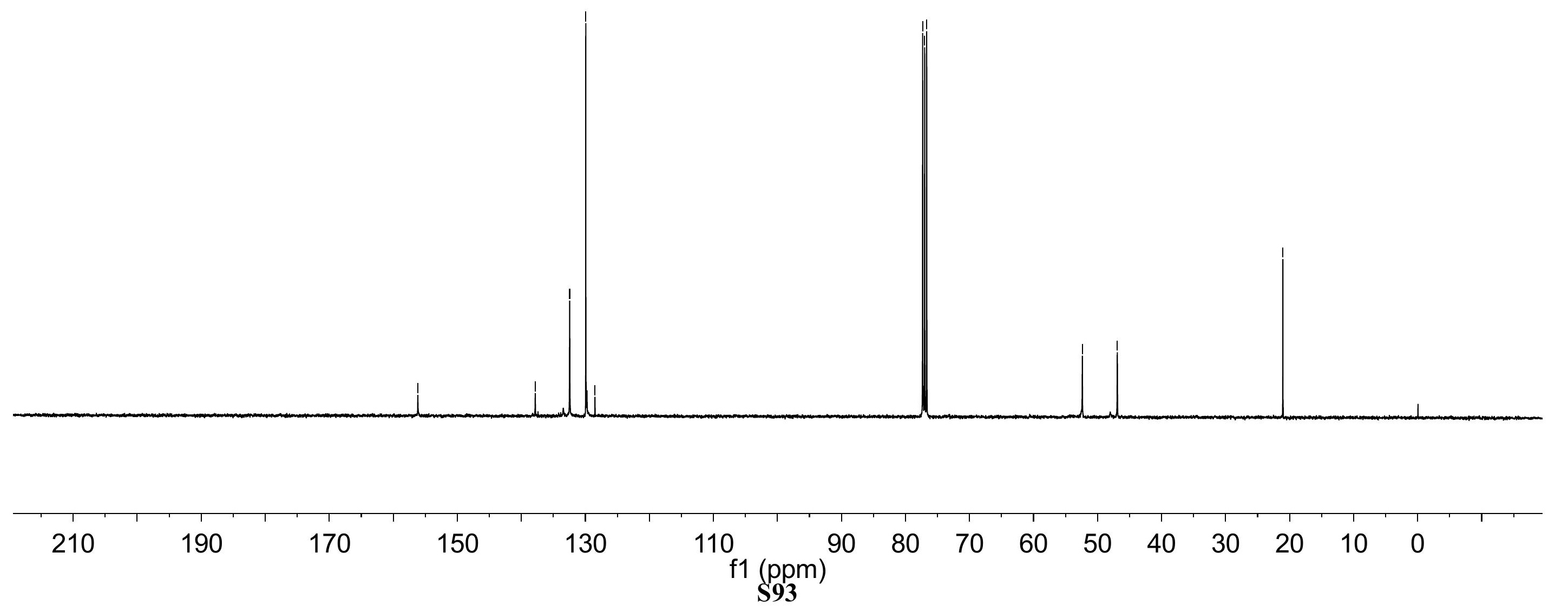


${ }^{1} \mathrm{H}$ NMR $\left(400 \mathrm{MHz}, \mathrm{CDCl}_{3}\right)$

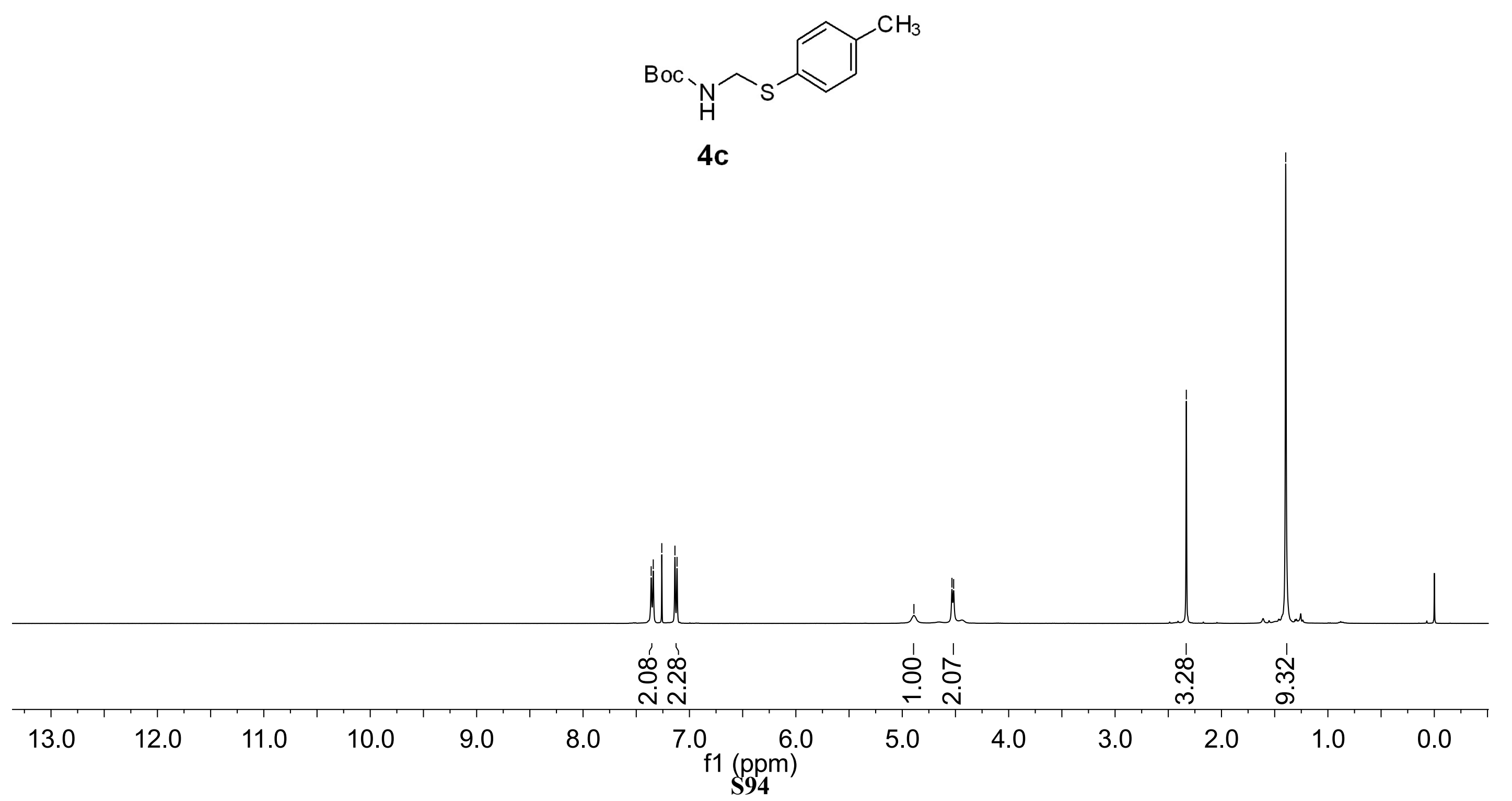


${ }^{13} \mathrm{C}\left\{{ }^{1} \mathrm{H}\right\} \operatorname{NMR}\left(101 \mathrm{MHz}, \mathrm{CDCl}_{3}\right)$

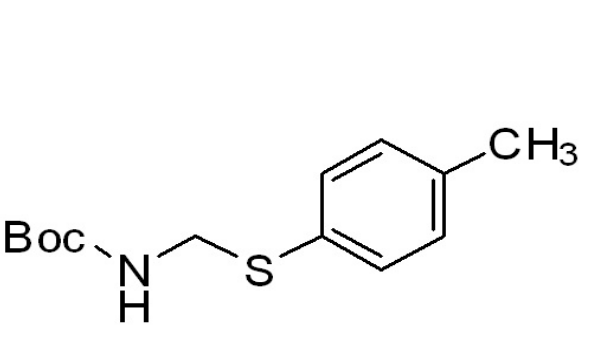

4c 
${ }^{1} \mathrm{H} \mathrm{NMR}\left(400 \mathrm{MHz}, \mathrm{CDCl}_{3}\right)$<smiles>Cc1ccc(SCNC(=O)c2ccc3ccccc3c2)cc1</smiles>

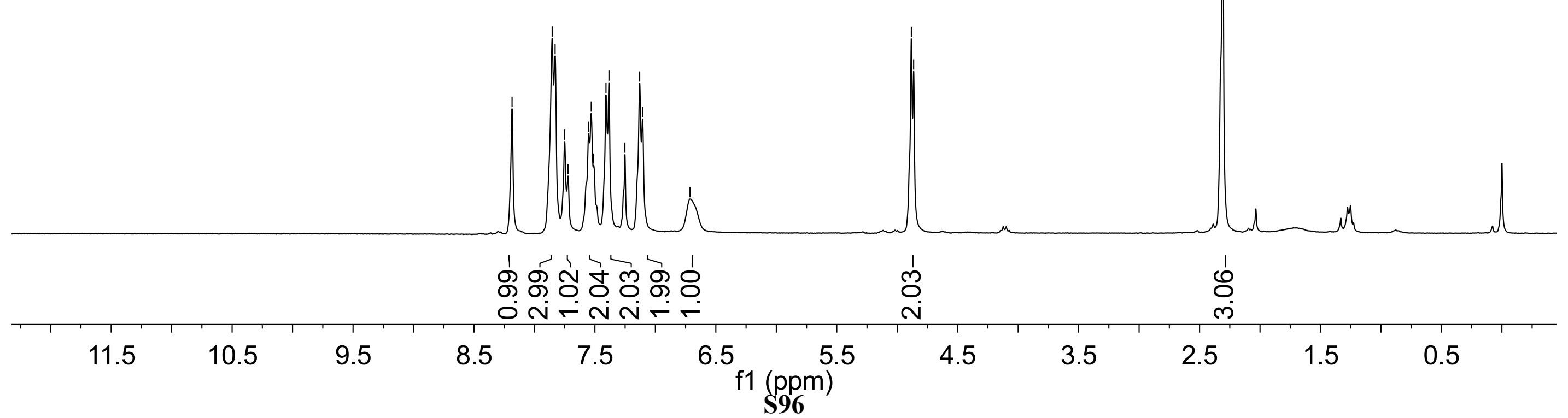


${ }^{13} \mathrm{C}\left\{{ }^{1} \mathrm{H}\right\} \operatorname{NMR}\left(101 \mathrm{MHz}, \mathrm{CDCl}_{3}\right)$

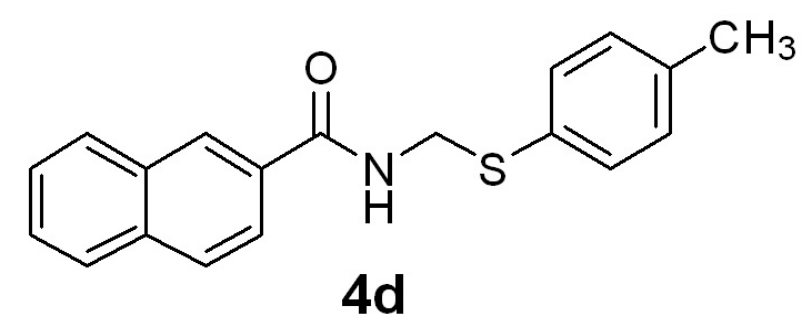


${ }^{1} \mathrm{H} \mathrm{NMR}\left(400 \mathrm{MHz}, \mathrm{CDCl}_{3}\right)$
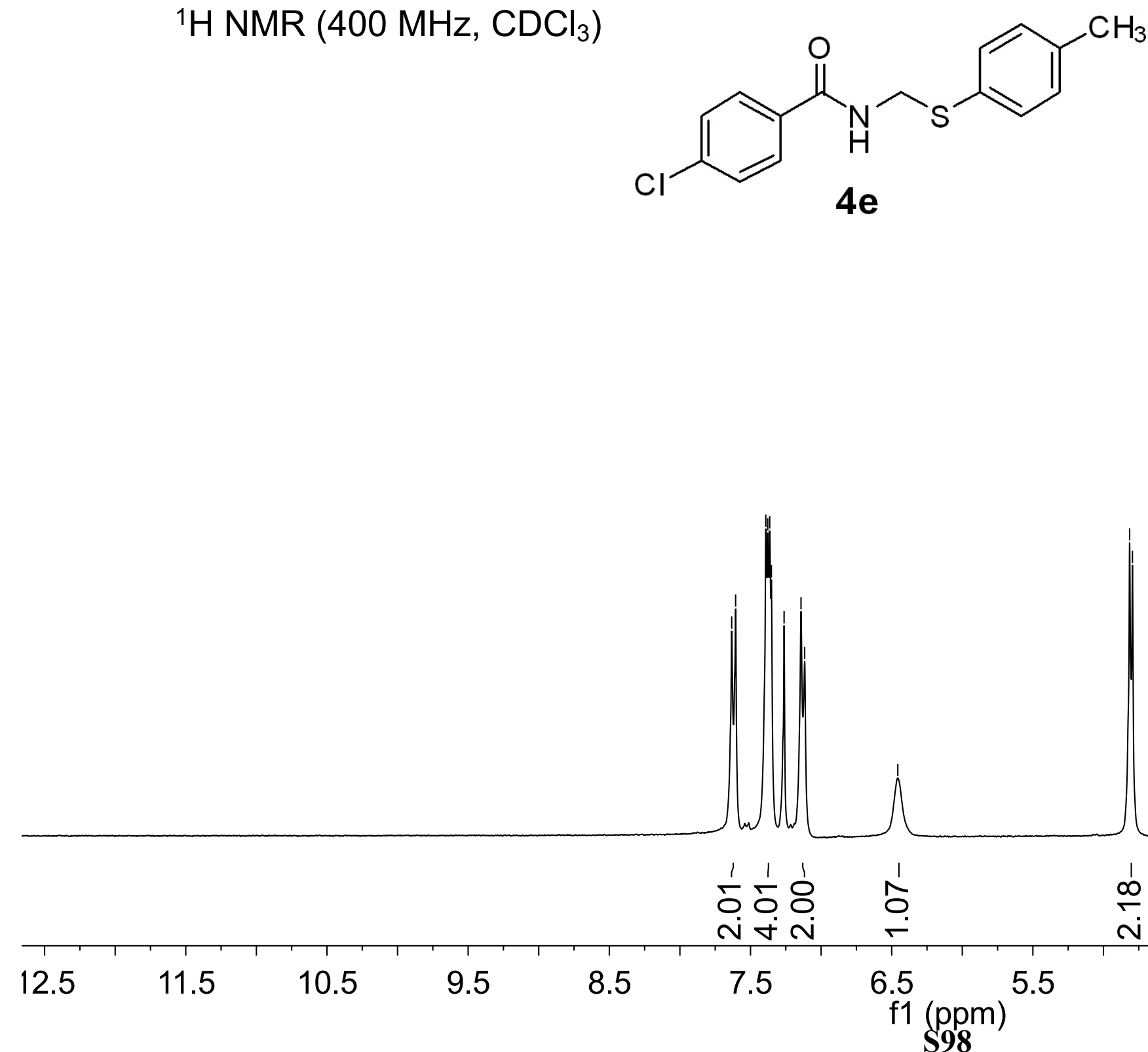

$\frac{1}{1}$

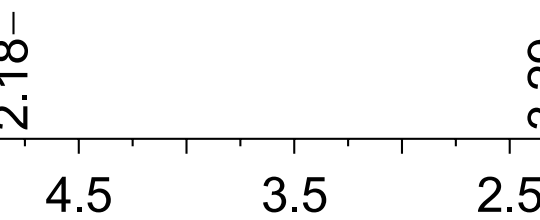

กั่ 
${ }^{13} \mathrm{C}\left\{{ }^{1} \mathrm{H}\right\}$ NMR (101 MHz, $\left.\mathrm{CDCl}_{3}\right)$<smiles>Cc1ccc(SCNC(=O)c2ccc(Cl)cc2)cc1</smiles> 
${ }^{1} \mathrm{H}$ NMR $\left(400 \mathrm{MHz}, \mathrm{CDCl}_{3}\right)$
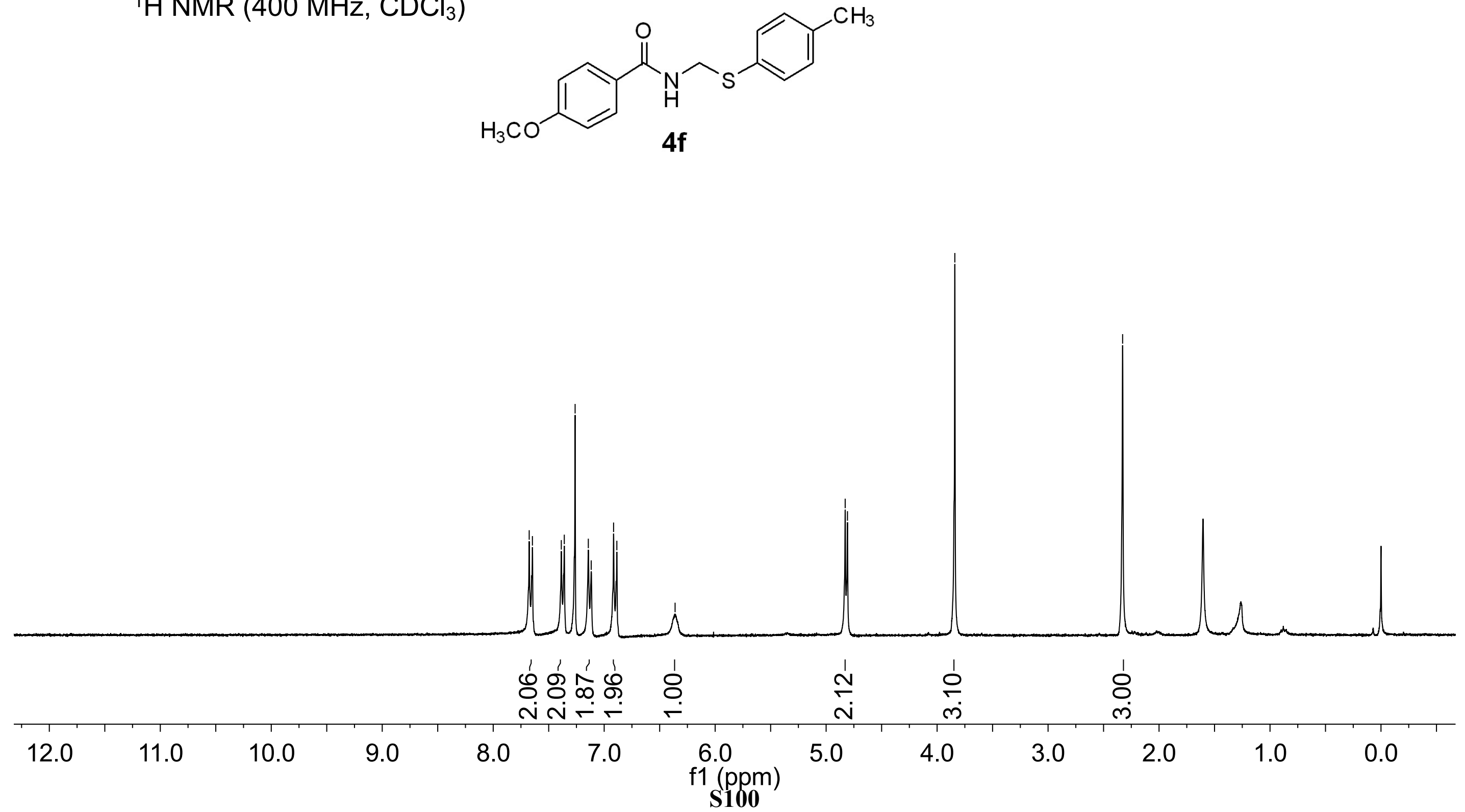
${ }^{13} \mathrm{C}\left\{{ }^{1} \mathrm{H}\right\}$ NMR $\left(101 \mathrm{MHz}, \mathrm{CDCl}_{3}\right)$<smiles>[Z11]CNC(=O)c1ccc(OC)cc1</smiles>
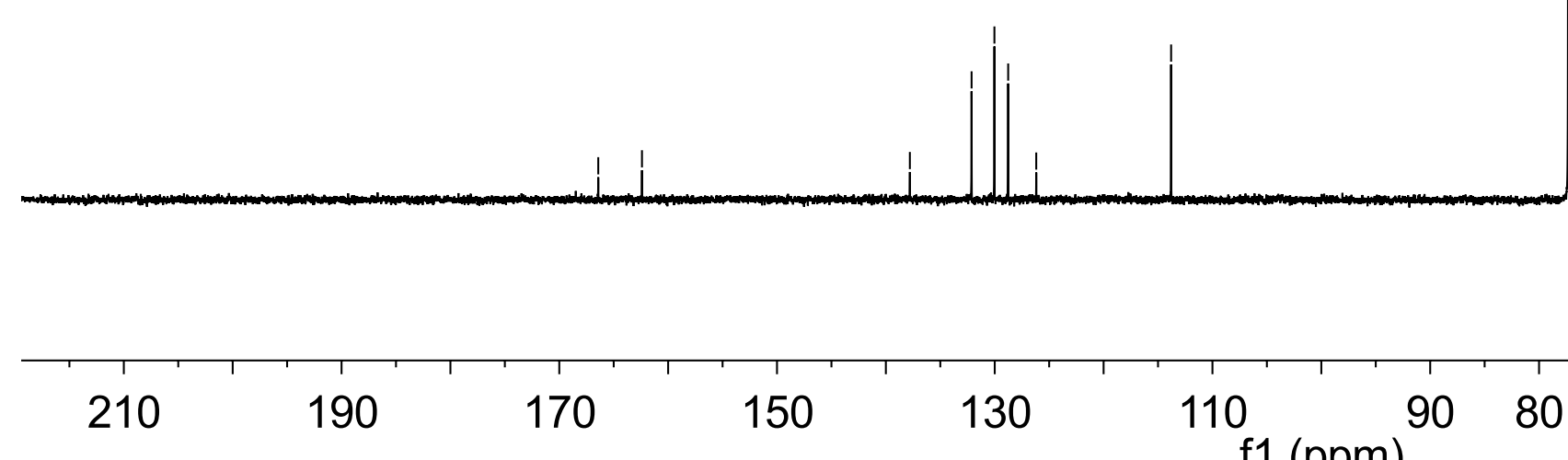
${ }^{1} \mathrm{H}$ NMR $\left(400 \mathrm{MHz}, \mathrm{CDCl}_{3}\right)$

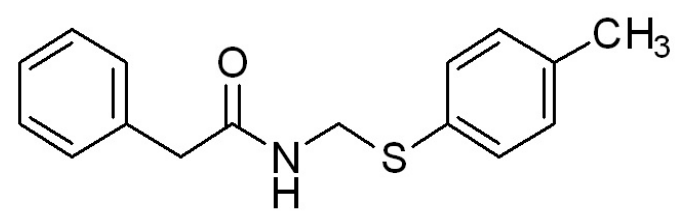

$4 g$

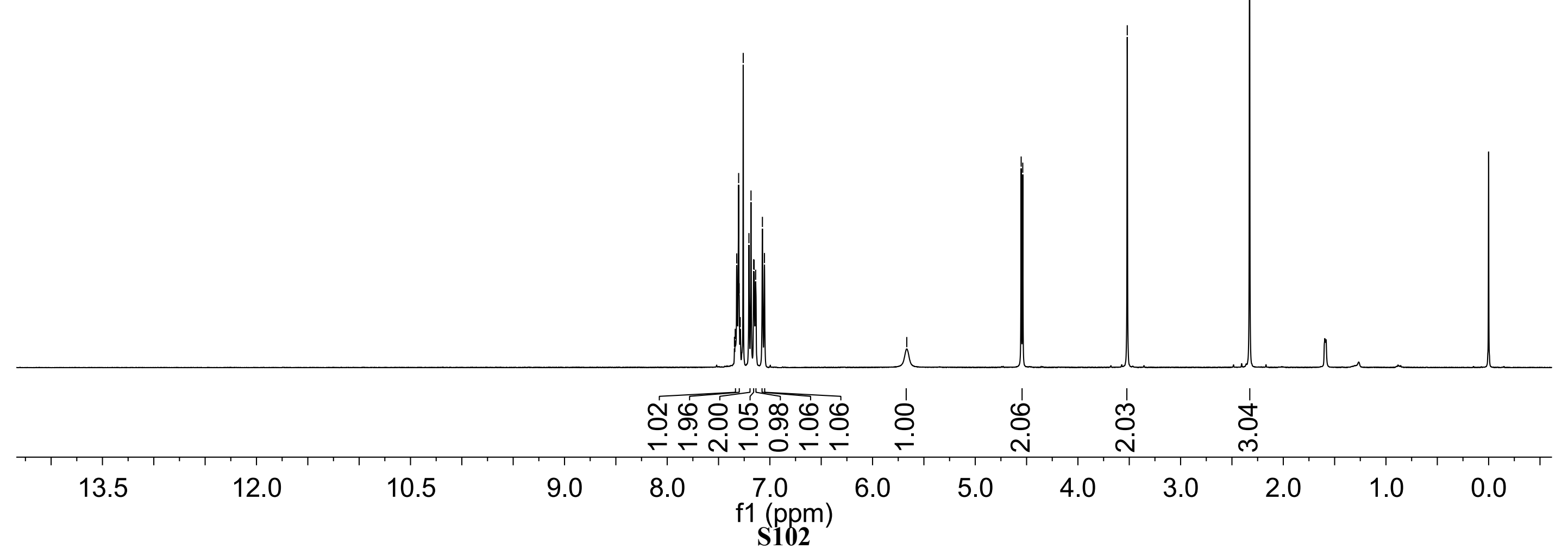



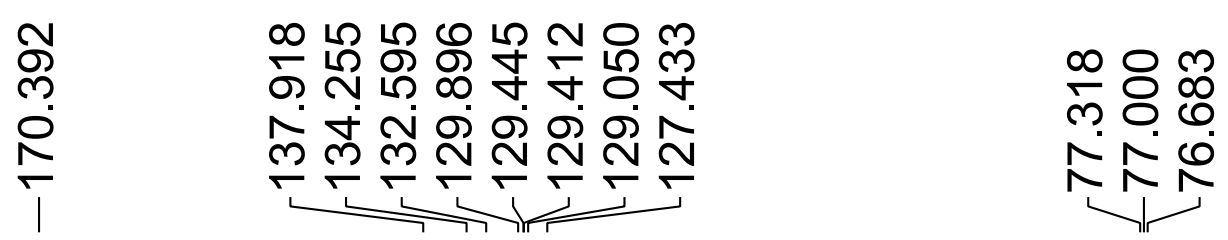

क

ํ.

${ }^{13} \mathrm{C}\left\{{ }^{1} \mathrm{H}\right\} \operatorname{NMR}\left(101 \mathrm{MHz}, \mathrm{CDCl}_{3}\right)$

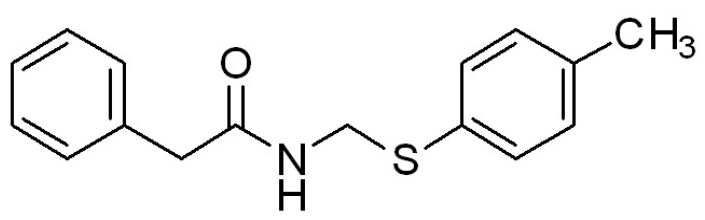

$4 \mathrm{~g}$

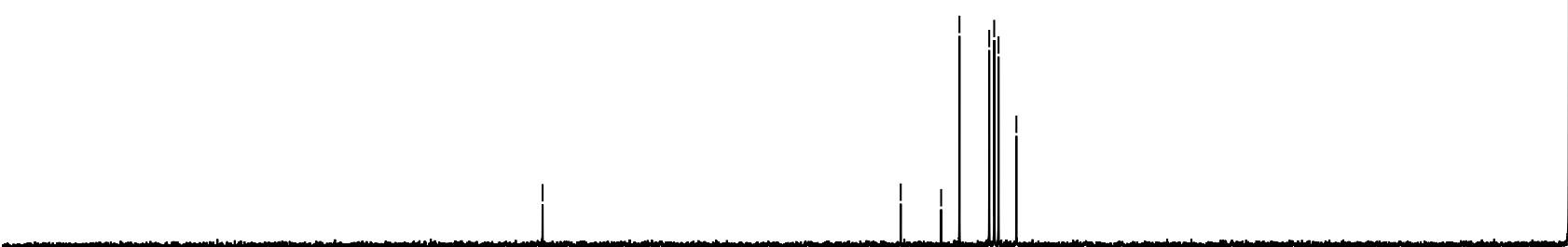

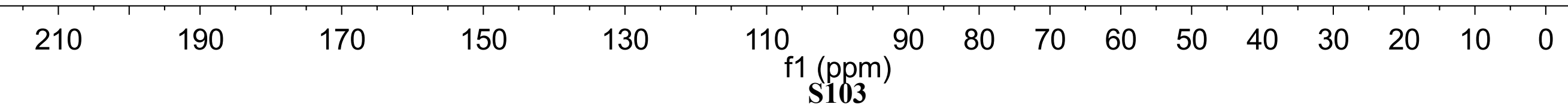


${ }^{1} \mathrm{H}$ NMR $\left(400 \mathrm{MHz}, \mathrm{CDCl}_{3}\right)$
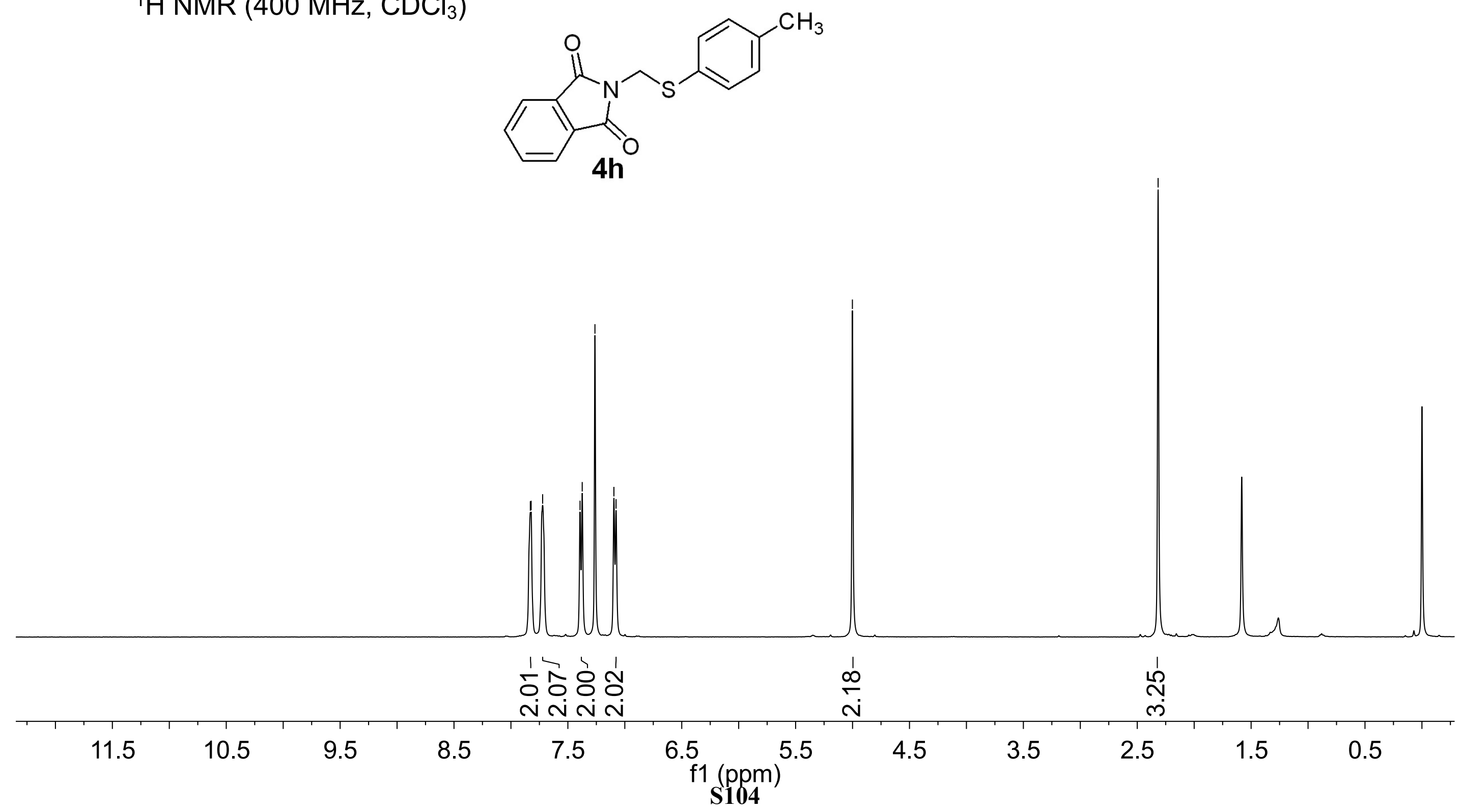
${ }^{13} \mathrm{C}\left\{{ }^{1} \mathrm{H}\right\} \operatorname{NMR}\left(101 \mathrm{MHz}, \mathrm{CDCl}_{3}\right)$
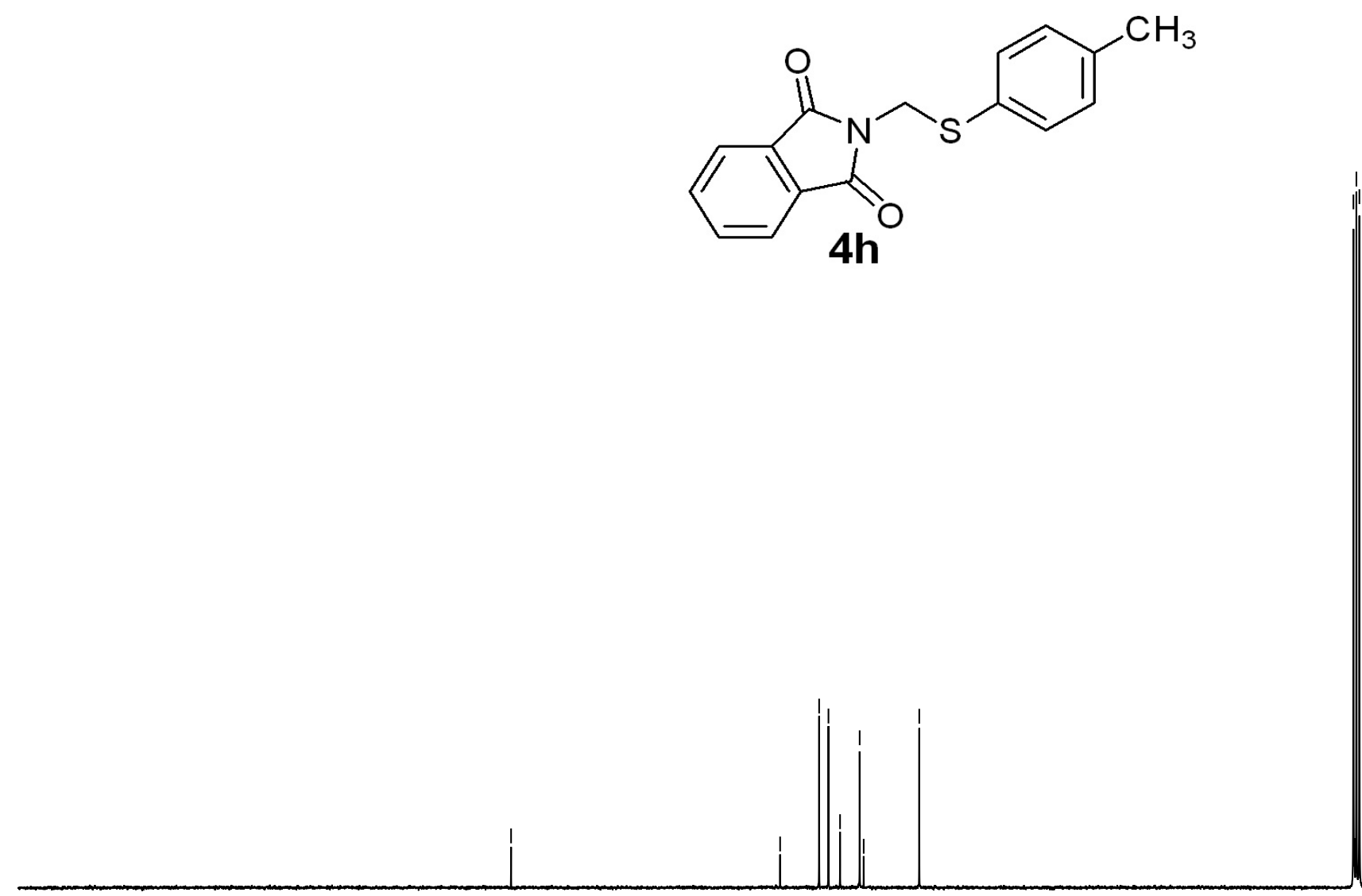

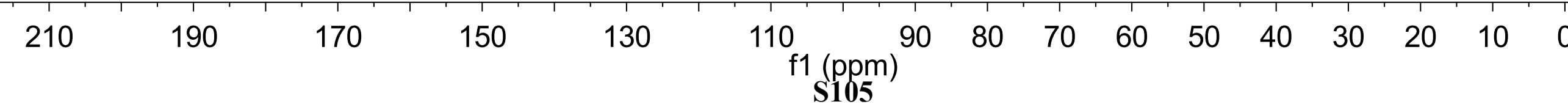


${ }^{1} \mathrm{H} \mathrm{NMR}\left(400 \mathrm{MHz}, \mathrm{CDCl}_{3}\right)$
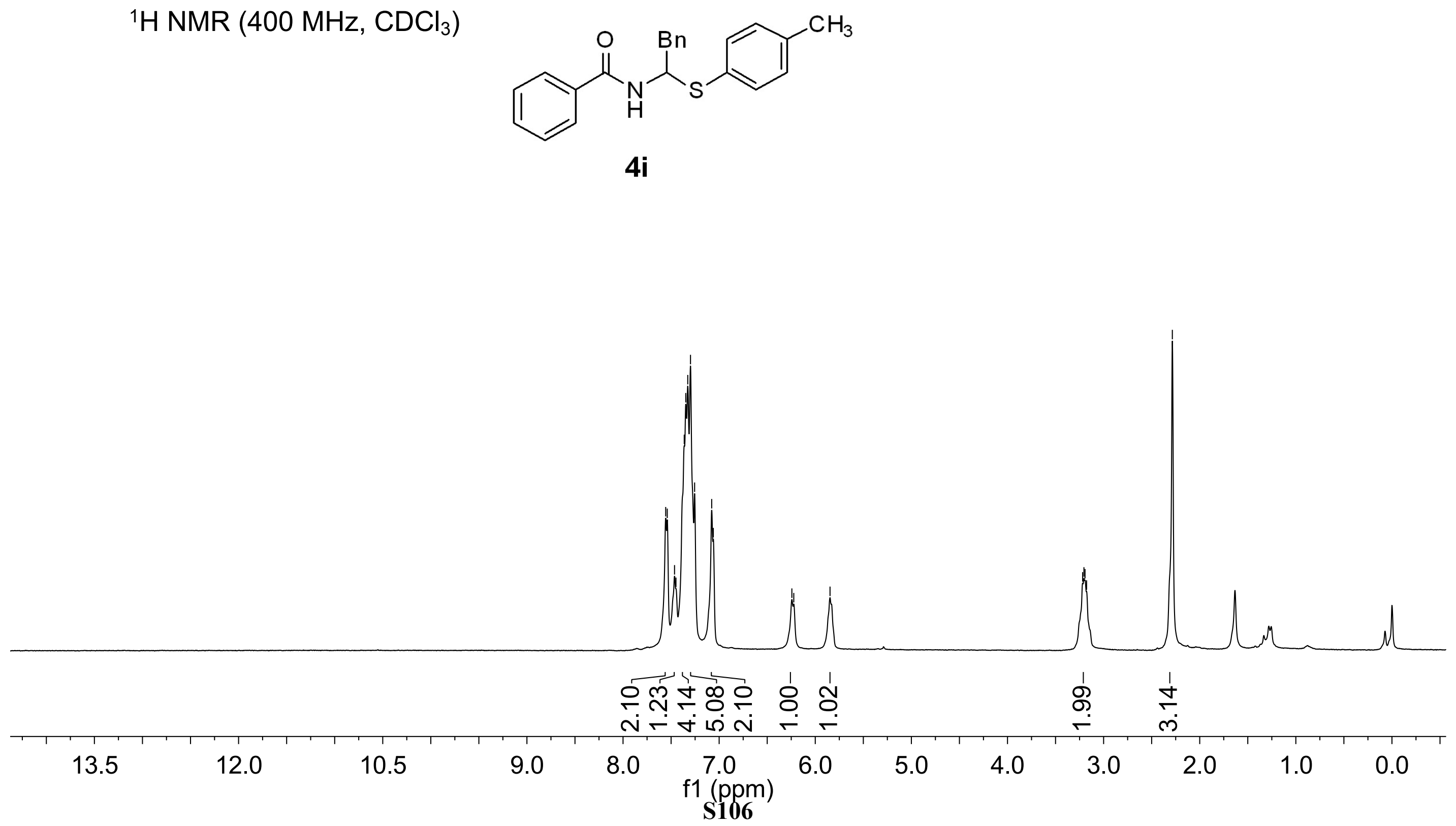
${ }^{13} \mathrm{C}\left\{{ }^{1} \mathrm{H}\right\}$ NMR $\left(101 \mathrm{MHz}, \mathrm{CDCl}_{3}\right)$<smiles>Cc1ccc(SC(Cc2ccccc2)NC(=O)c2ccccc2)cc1</smiles>

$4 \mathbf{i}$

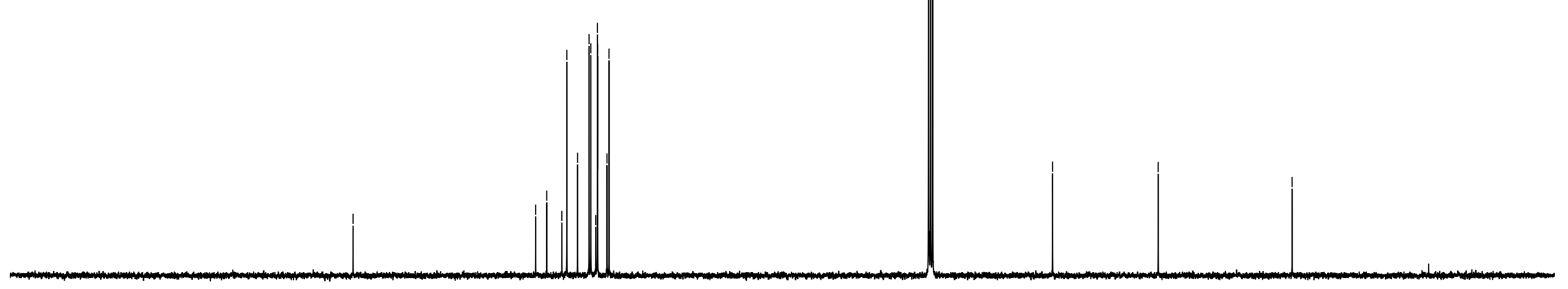

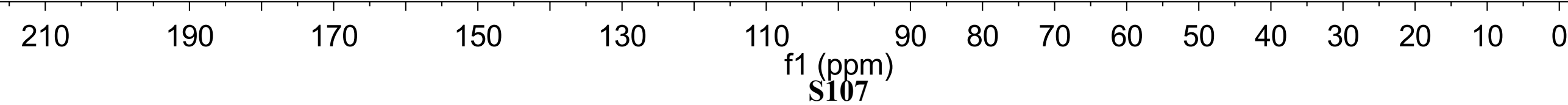


${ }^{1} \mathrm{H}$ NMR $\left(400 \mathrm{MHz}, \mathrm{CDCl}_{3}\right)$
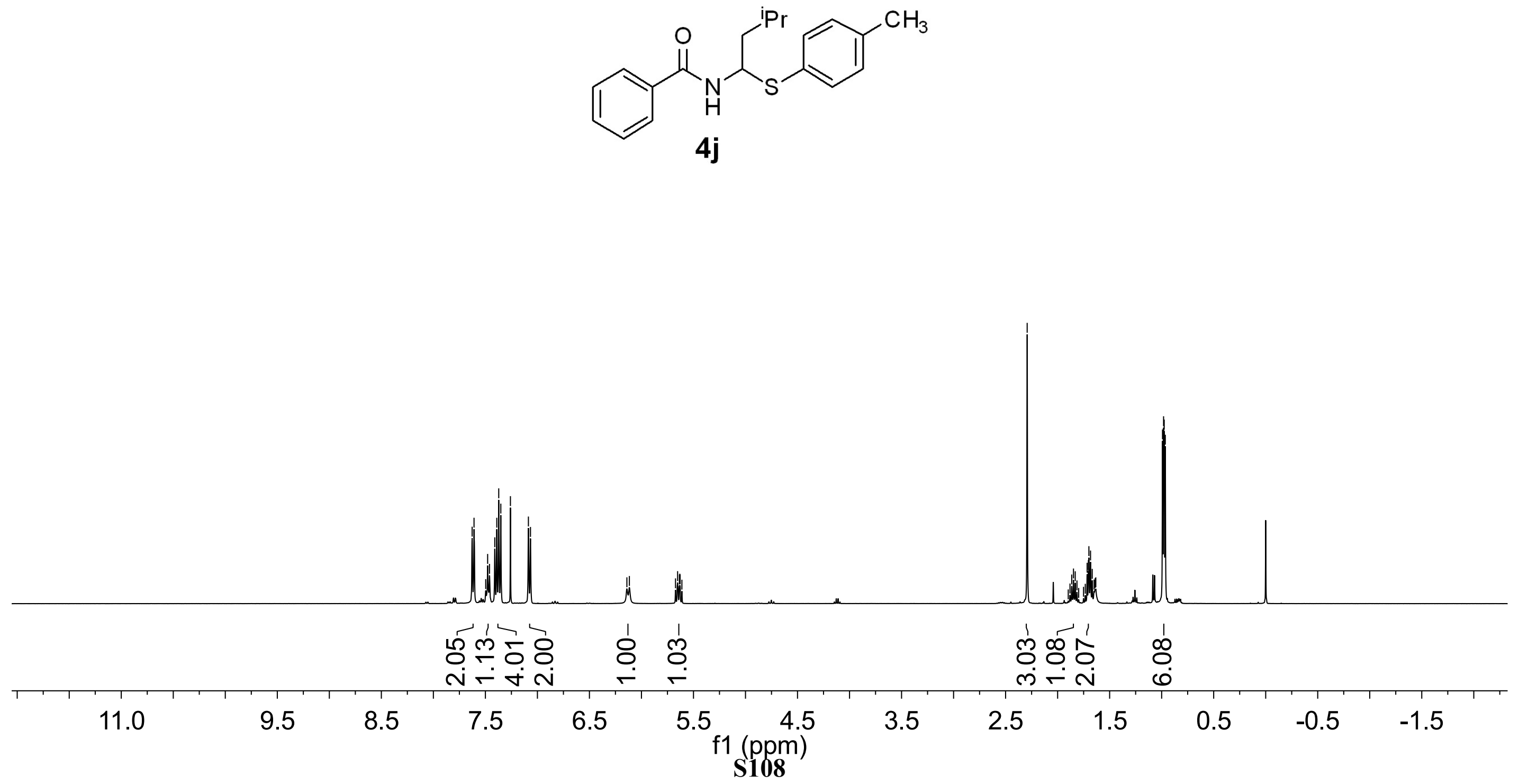
${ }^{13} \mathrm{C}\left\{{ }^{1} \mathrm{H}\right\}$ NMR $\left(101 \mathrm{MHz}, \mathrm{CDCl}_{3}\right)$

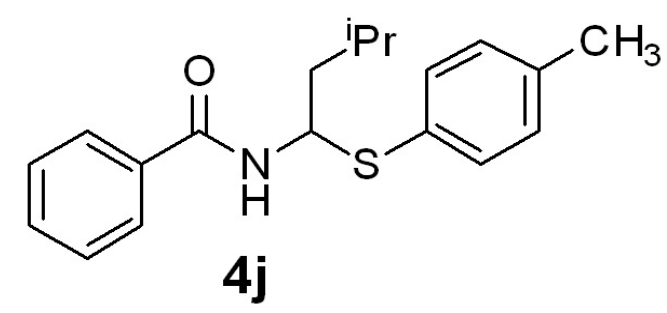

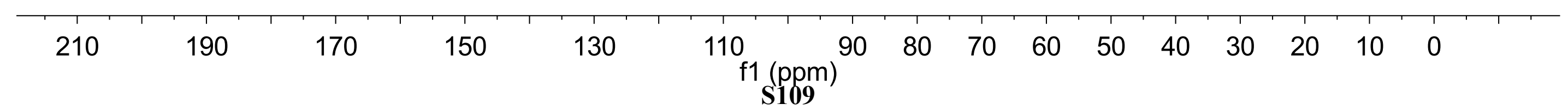


${ }^{1} \mathrm{H}$ NMR $\left(400 \mathrm{MHz}, \mathrm{CDCl}_{3}\right)$
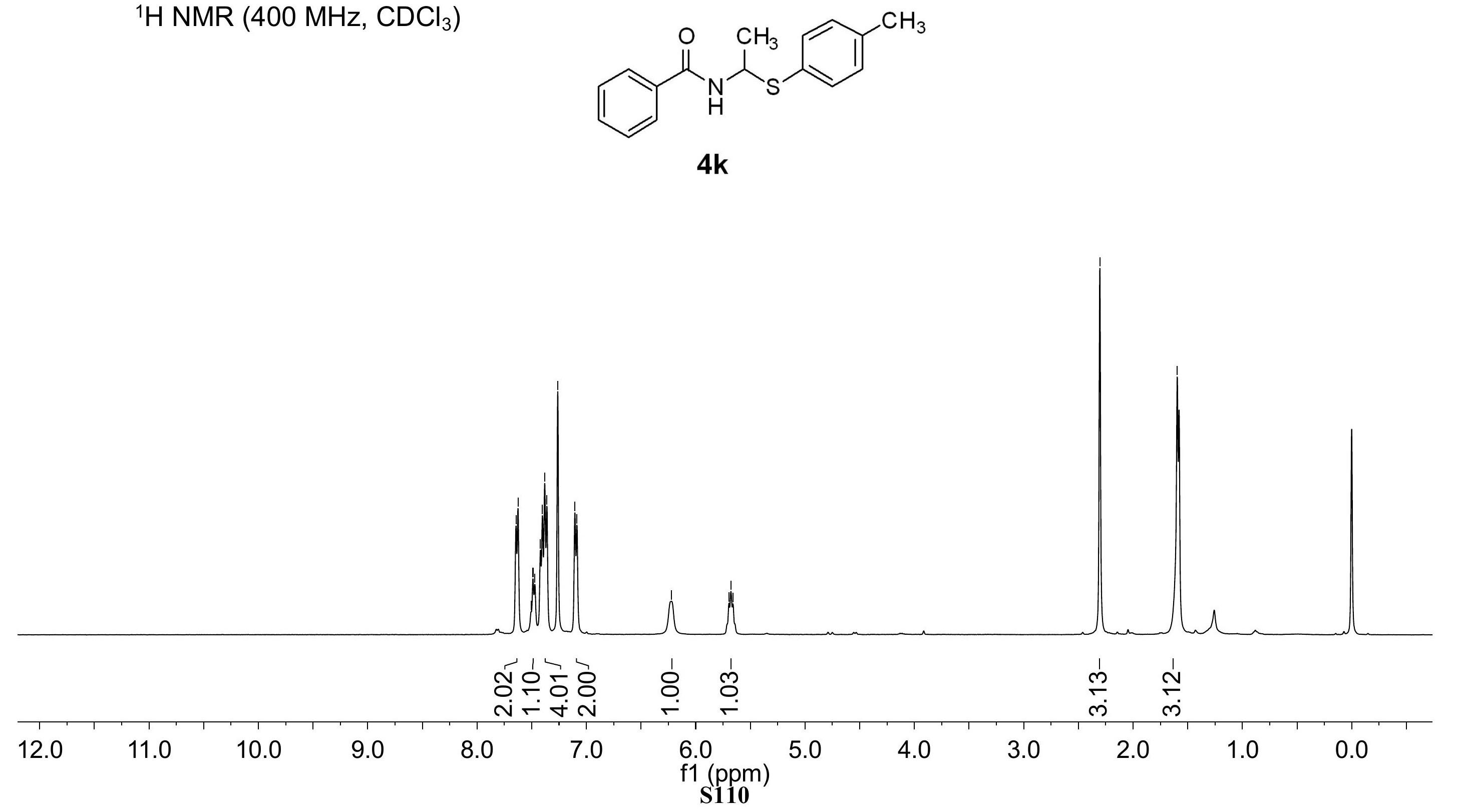
${ }^{13} \mathrm{C}\left\{{ }^{1} \mathrm{H}\right\} \operatorname{NMR}\left(101 \mathrm{MHz}, \mathrm{CDCl}_{3}\right)$<smiles>Cc1ccc(SC(C)NC(=O)c2ccccc2)cc1</smiles>
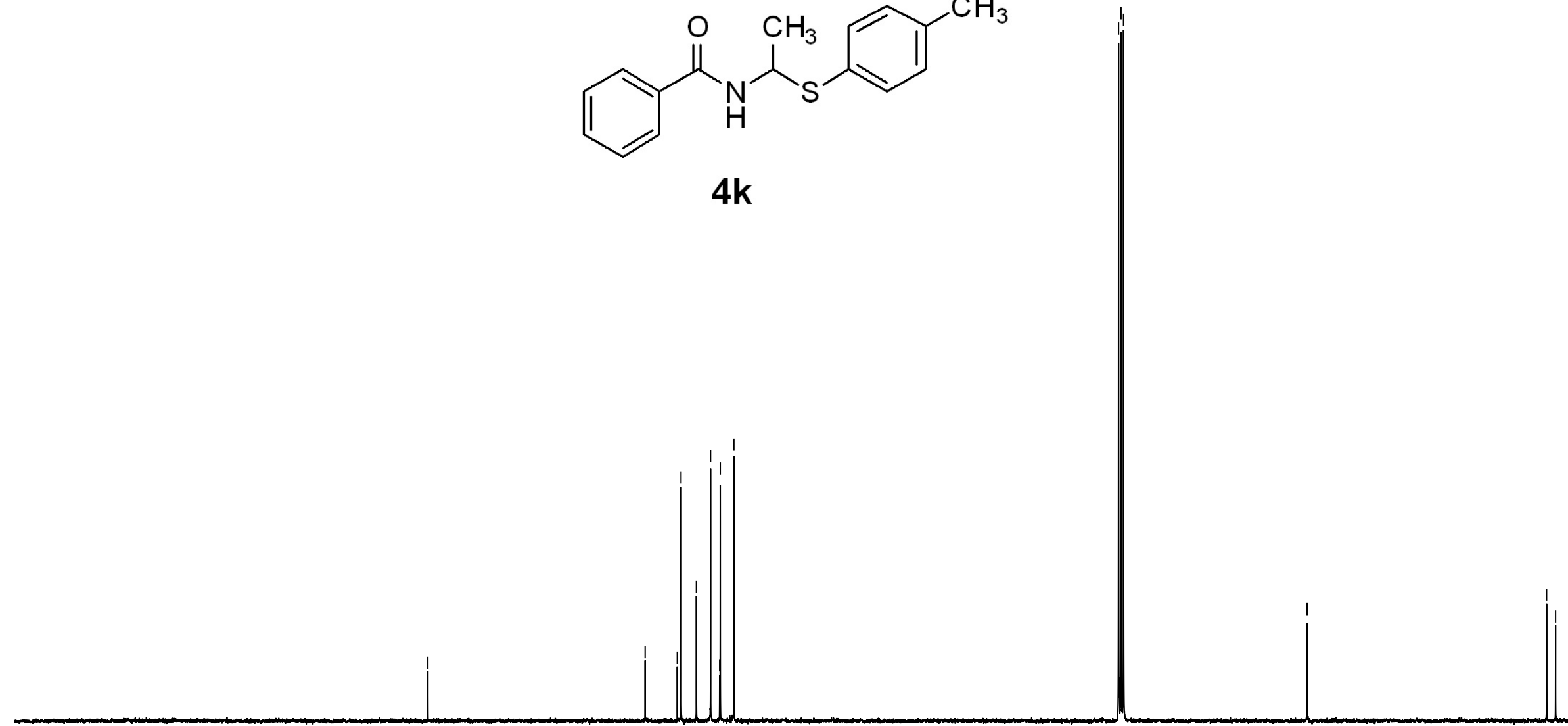


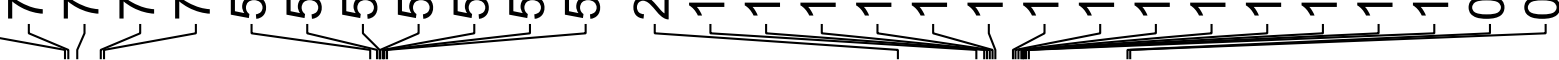

${ }^{1} \mathrm{H}$ NMR $\left(400 \mathrm{MHz}, \mathrm{CDCl}_{3}\right)$

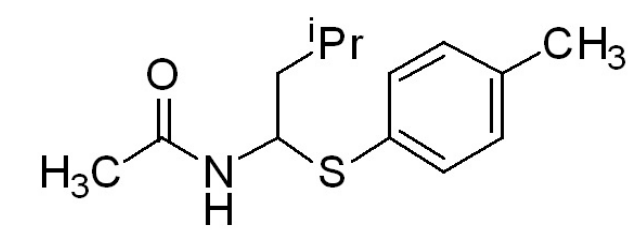

4!

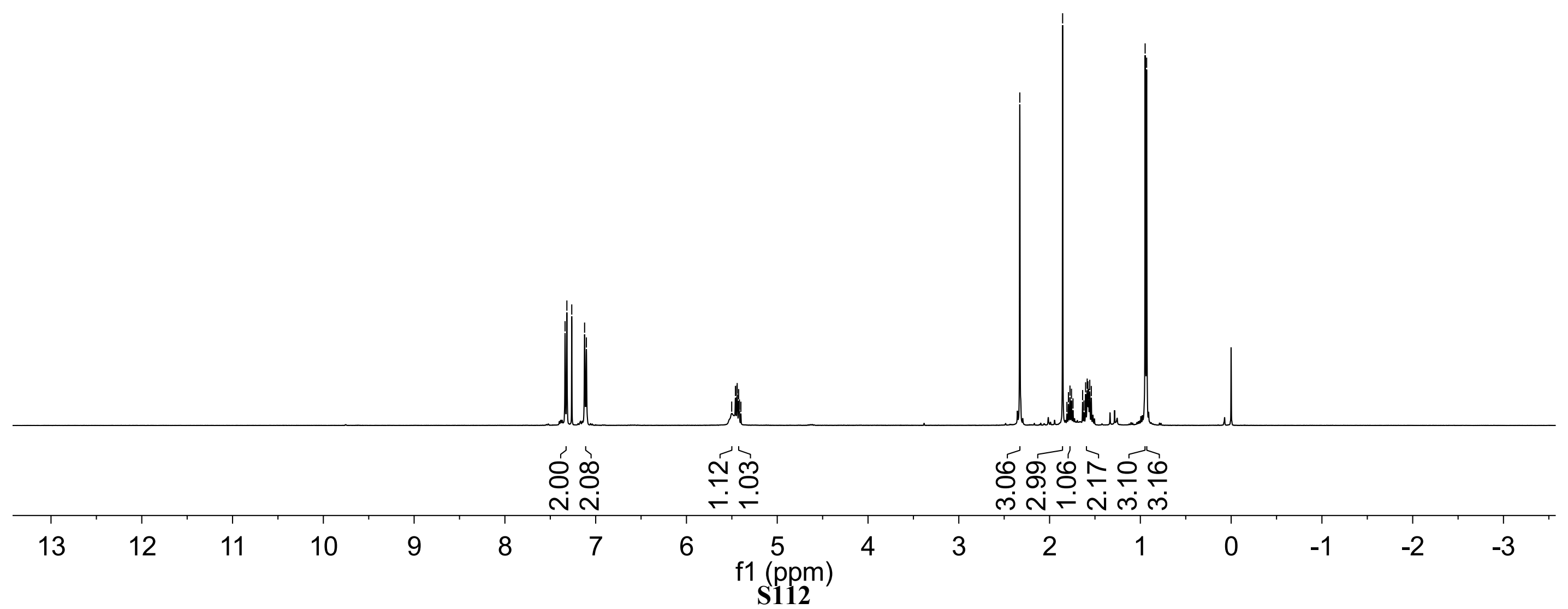


${ }^{13} \mathrm{C}\left\{{ }^{1} \mathrm{H}\right\}$ NMR (101 MHz, CDCl 3 )<smiles>CC(=O)NC(CC(C)C)Sc1ccc(C)cc1</smiles>

4!

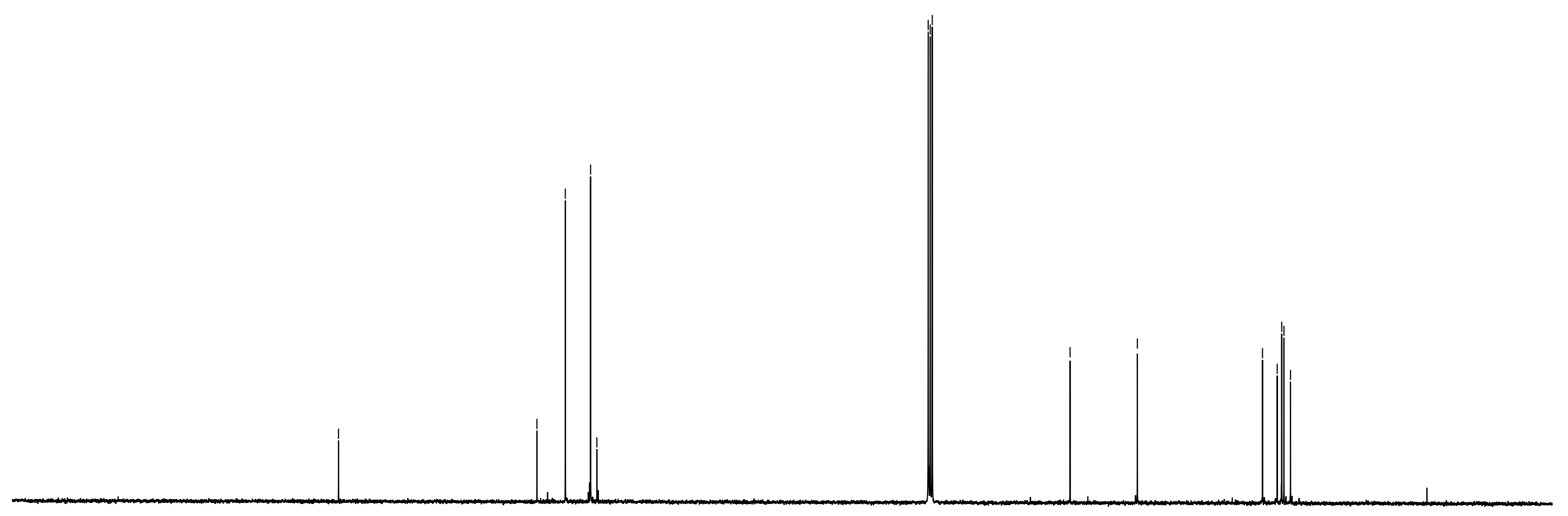


${ }^{1} \mathrm{H}$ NMR $\left(400 \mathrm{MHz}, \mathrm{CDCl}_{3}\right)$
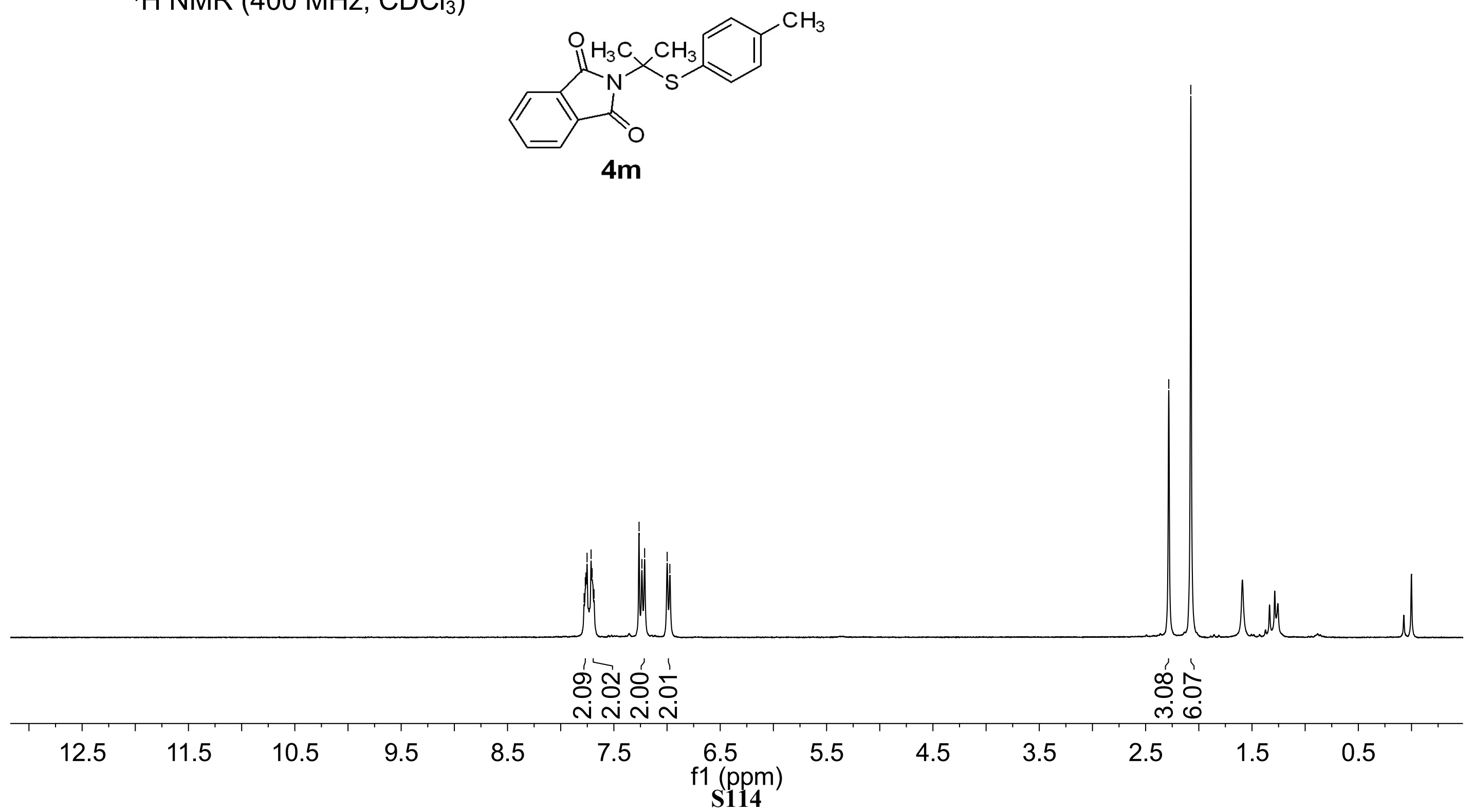
${ }^{13} \mathrm{C}\left\{{ }^{1} \mathrm{H}\right\} \operatorname{NMR}\left(101 \mathrm{MHz}, \mathrm{CDCl}_{3}\right)$

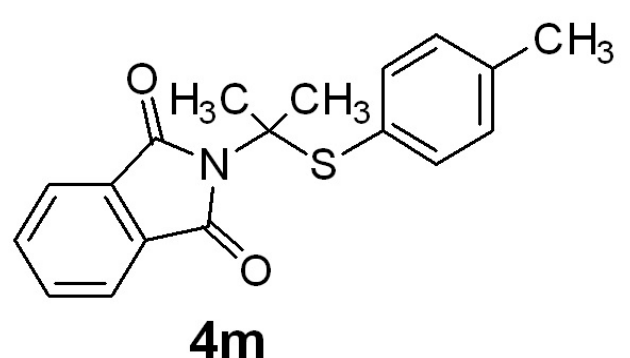

$4 m$

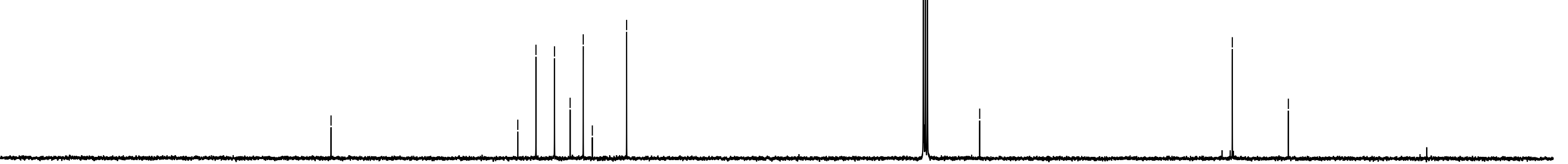

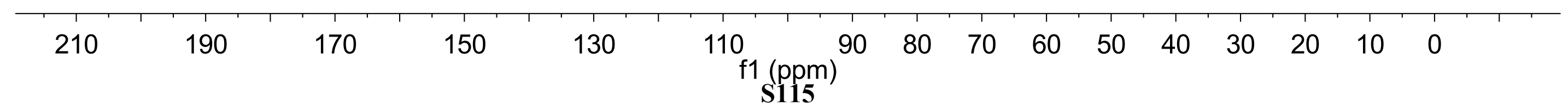




\section{$\frac{a}{\frac{9}{5}}$}

${ }^{19} \mathrm{~F}\left\{{ }^{1} \mathrm{H}\right\} \operatorname{NMR}\left(377 \mathrm{MHz}, \mathrm{CDCl}_{3}\right)$

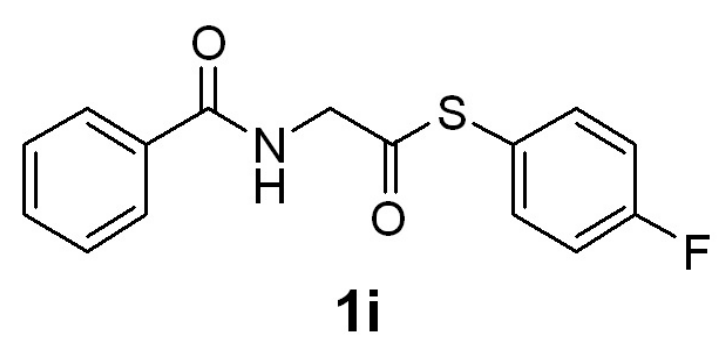

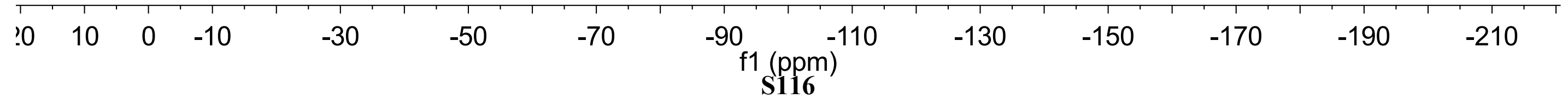


${ }^{19} \mathrm{~F}\left\{{ }^{1} \mathrm{H}\right\} \mathrm{NMR}\left(377 \mathrm{MHz}, \mathrm{CDCl}{ }_{3}\right)$

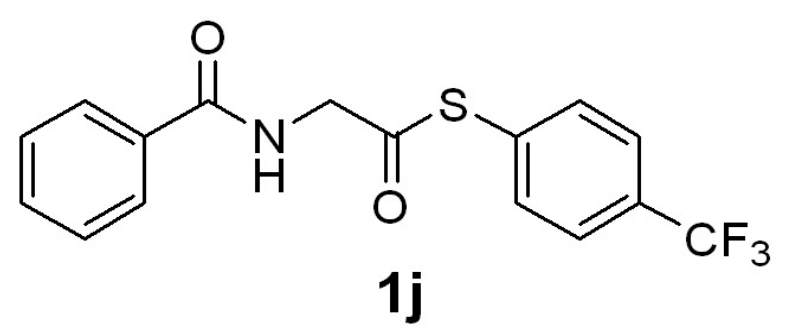

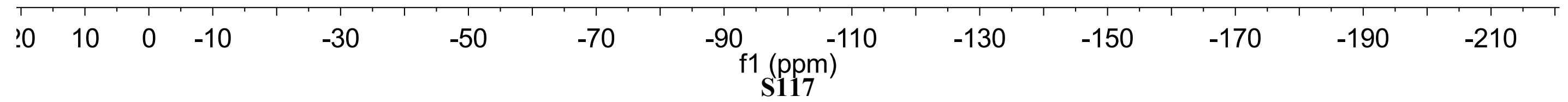




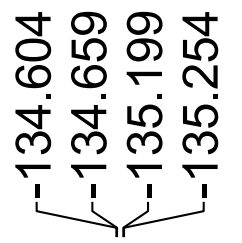

${ }^{19} \mathrm{~F}\left\{{ }^{1} \mathrm{H}\right\} \mathrm{NMR}\left(377 \mathrm{MHz}, \mathrm{CDCl}_{3}\right)$<smiles>O=C(CNC(=O)c1ccccc1)Sc1ccc(F)c(F)c1</smiles>

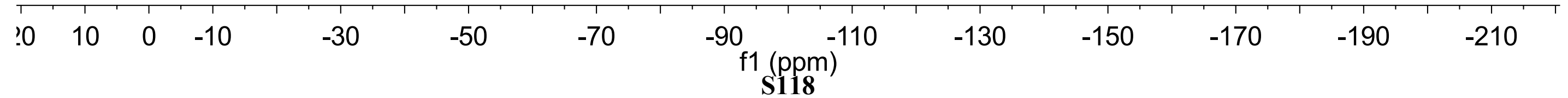




\section{$\frac{a}{\frac{9}{5}}$}

${ }^{19} \mathrm{~F}\left\{{ }^{1} \mathrm{H}\right\} \operatorname{NMR}\left(377 \mathrm{MHz}, \mathrm{CDCl}_{3}\right)$<smiles>O=C(NCSc1ccc(F)cc1)c1ccccc1</smiles> 
${ }^{19} \mathrm{~F}\left\{{ }^{1} \mathrm{H}\right\} \mathrm{NMR}\left(377 \mathrm{MHz}, \mathrm{CDCl}_{3}\right)$

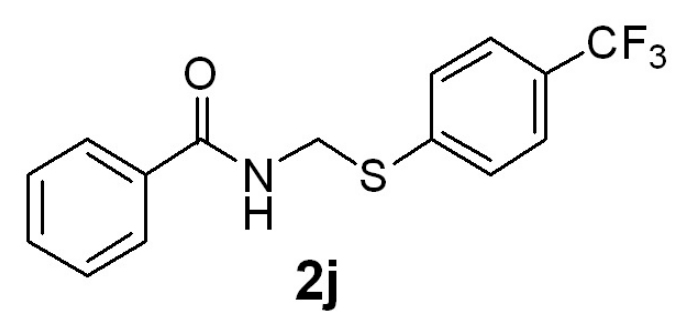

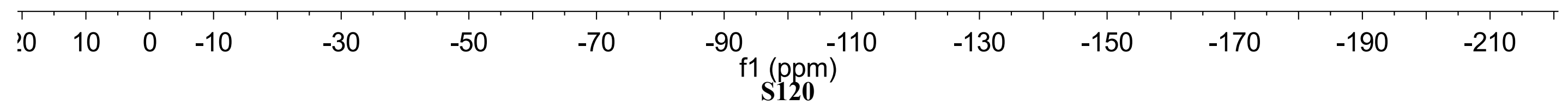


${ }^{19} \mathrm{~F}\left\{{ }^{1} \mathrm{H}\right\} \mathrm{NMR}\left(377 \mathrm{MHz}, \mathrm{CDCl}_{3}\right)$<smiles>O=C(NCSc1ccc(F)c(F)c1)c1ccccc1</smiles>

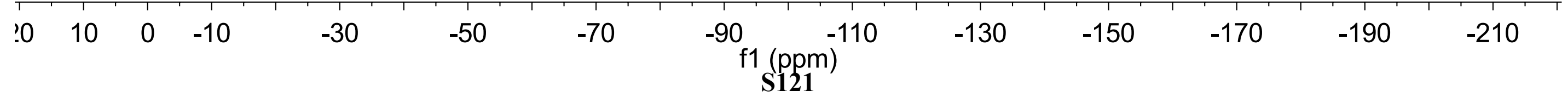


Multiple Mass Analysis: 4 mass(es) processed - displaying only valid results

Tolerance $=5.0$ PPM / DBE: $\min =-1.5, \max =50.0$

Element prediction: Off

Monoisotopic Mass, Odd and Even Electron Ions

41 formula(e) evaluated with 2 results within limits (all results (up to 1000) for each mass)

Elements Used:

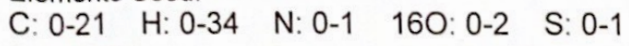

DEFAULT

Y $21-430-\mathrm{Cl} 251(4.183) \mathrm{Cm}(249: 251-(22: 41+73: 81))$
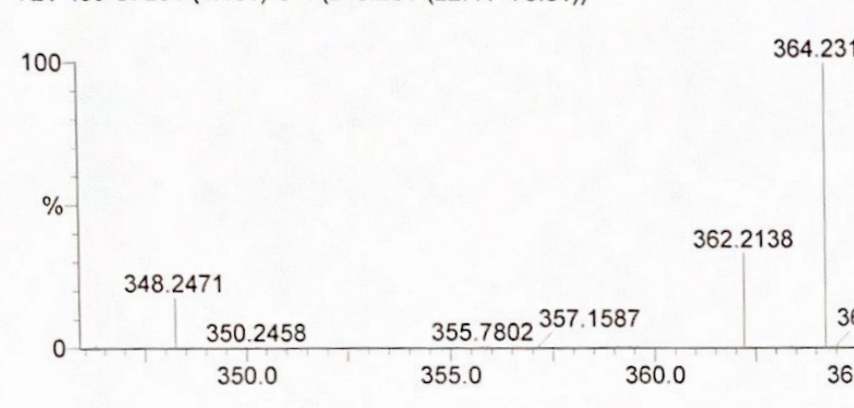

Minimum: $\quad 5.00$

Maximum: $\quad 100.00$

360.0

4.2311

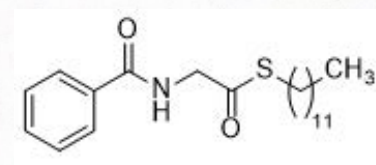

TOF MS Cl+

$3.87 e+002$

\begin{tabular}{|c|c|c|c|c|c|c|c|c|c|c|}
\hline Mass & RA & Calc. Mass & $\mathrm{mDa}$ & PPM & $\mathrm{DBE}$ & i-FIT & Eorn & גa & & \\
\hline $\begin{array}{l}362.2138 \\
364.2311\end{array}$ & $\begin{array}{l}33.19 \\
100.00\end{array}$ & $\begin{array}{l}362.2154 \\
364.2310\end{array}$ & $\begin{array}{l}-1.6 \\
0.1\end{array}$ & $\begin{array}{l}-4.4 \\
0.3\end{array}$ & $\begin{array}{l}6.5 \\
5.5\end{array}$ & $\begin{array}{l}2773260.3 \\
5546207.0\end{array}$ & $\begin{array}{l}\mathrm{C} 21 \\
\mathrm{C} 21\end{array}$ & $\begin{array}{l}\text { H32 } \\
\text { H34 }\end{array}$ & $\begin{array}{l}\mathrm{N} \\
\mathrm{N}\end{array}$ & $\begin{array}{l}1602 \\
1602\end{array}$ \\
\hline
\end{tabular}




\section{Elemental Composition Report}

Multiple Mass Analysis: 2 mass(es) processed - displaying only valid results

Tolerance $=5.0$ PPM / DBE: $\min =-1.5, \max =50.0$

Element prediction: Off

Monoisotopic Mass, Odd and Even Electron Ions

229 formula(e) evaluated with 1 results within limits (all results (up to 1000) for each mass)

Elements Used:

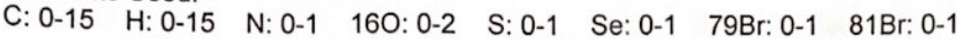

DEFAULT

1027-1 198 (3.300) Cm (198:199-(67:72+46:52))
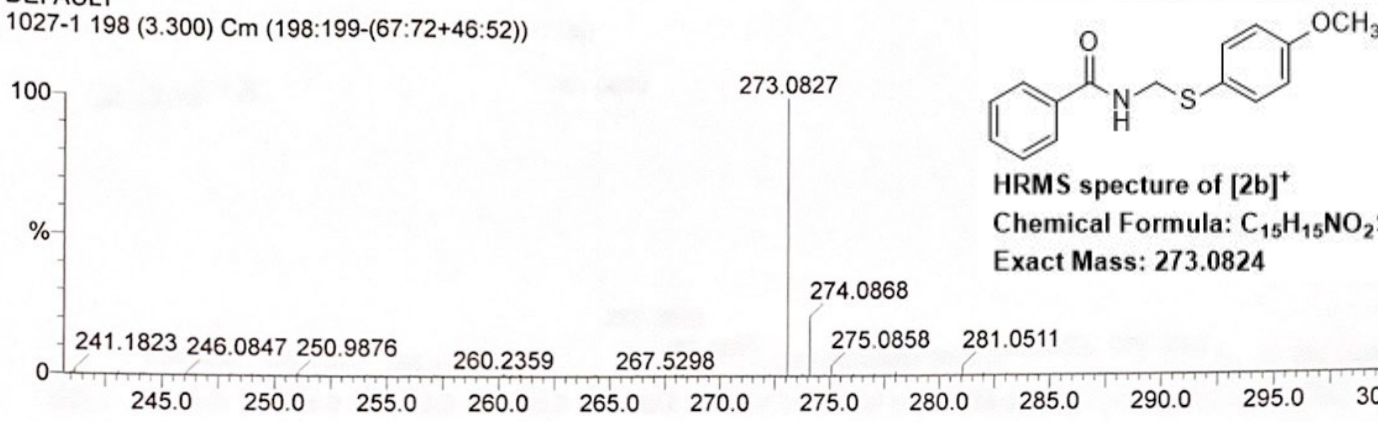

TOF MS EI+

$5.29 \mathrm{e}+002$

Minimum: $\quad 10.00$

Maximum: $\quad 100.00$

$\begin{array}{lll}30.0 & 5.0 & -1.5 \\ & 50.0\end{array}$

Mass

$\mathrm{RA}$

Calc. Mass

$\mathrm{mDa}$

PPM

$\mathrm{DBE}$

i-FIT

Formula

273.0827

100.00

273.0824

0.3

1.1

9.0

8.7

C15 H15 N 1602 S

HRMS specture of [2b]

Chemical Formula: $\mathrm{C}_{15} \mathrm{H}_{15} \mathrm{NO}_{2} \mathrm{~S}^{+}$

Exact Mass: 273.0824

274.0868

$302.1107 \quad 308.3471$

$\begin{array}{lllll} & 302.1107 & 308.3471 \\ 285.0 & 290.0 & 295.0 & 300.0 & 305.0\end{array}$


Multiple Mass Analysis: 2 mass(es) processed - displaying only valid results

Tolerance $=5.0$ PPM / DBE: $\min =-1.5, \max =50.0$

Element prediction: Off

Monoisotopic Mass, Odd and Even Electron lons

19 formula(e) evaluated with 1 results within limits (all results (up to 1000) for each mass)

Elements Used:

$\begin{array}{llllll}\text { C: } 0-18 & H: 0-21 & \text { B: } 0-1 & \text { N: } 0-1 & 160: 0-1 & \text { S: } 0-1\end{array}$

DEFAULT

1220-3 258 (4.300) Cm (258:261-(141:143+131:136))

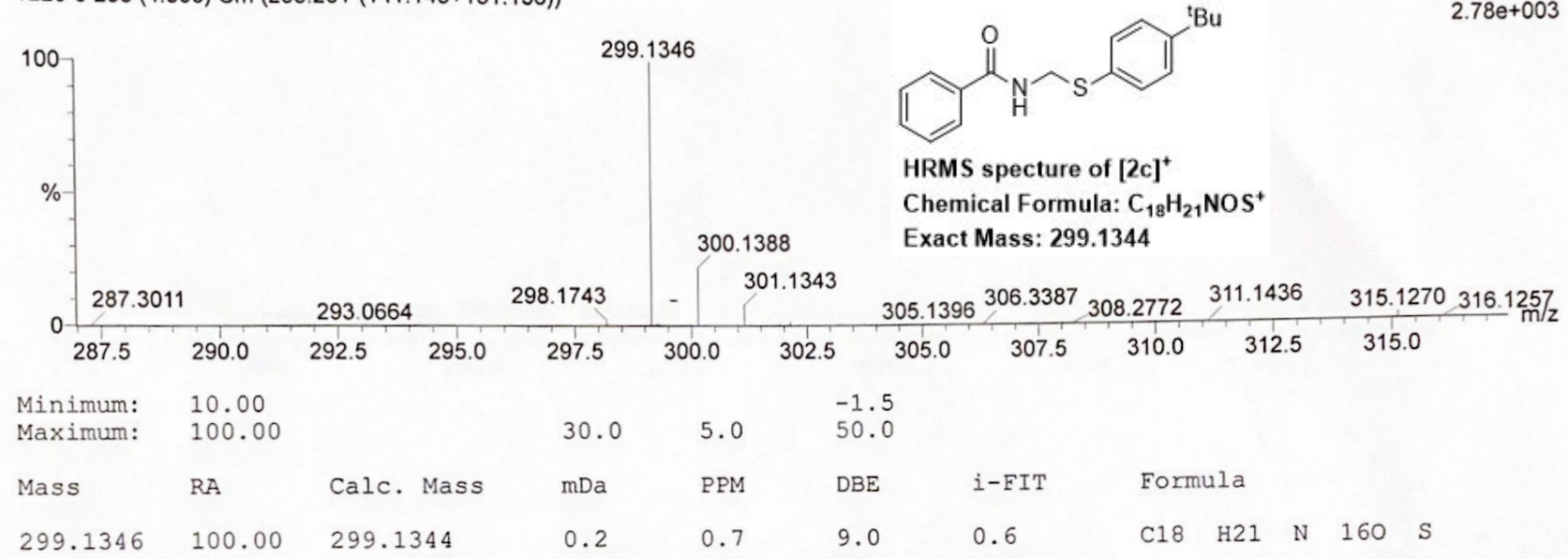


Multiple Mass Analysis: 2 mass(es) processed - displaying only valid results

Tolerance $=5.0$ PPM / DBE: $\min =-1.5, \max =50.0$

Element prediction: Off

Monoisotopic Mass, Odd and Even Electron Ions

19 formula(e) evaluated with 1 results within limits (all results (up to 1000) for each mass)

Elements Used:

$\begin{array}{llllll}\text { C: } 0-17 & \text { H: } 0-19 & \text { B: } 0-1 & \text { N: }: 0-1 & \text { 160: } 0-1 & \text { S: } 0-1\end{array}$

DEFAULT

$14-1268(4.467) \mathrm{Cm}(264: 270-(137: 147+116: 128))$

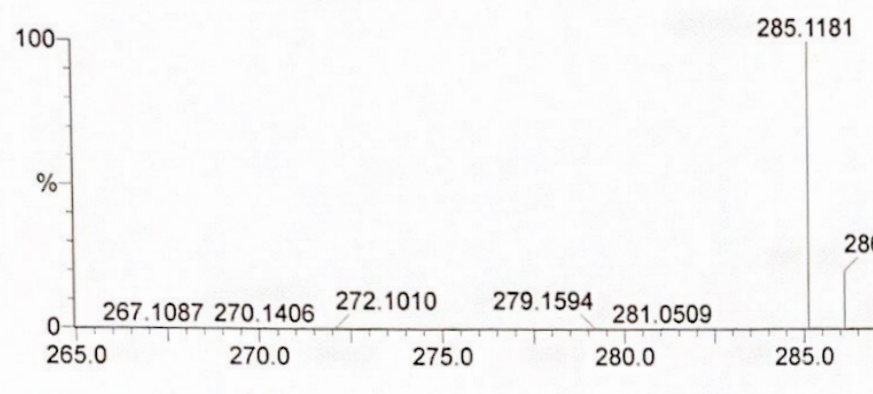

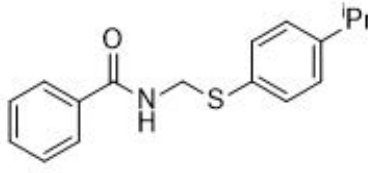

HRMS specture of [2d] $]^{+}$

Chemical Formula: $\mathrm{C}_{17} \mathrm{H}_{19} \mathrm{NOS}^{+}$

286.1224

Exact Mass: 285.1187

Minimum: $\quad 10.00$

Maximum: $\quad 100.00$

30.0

5.0

$-1.5$

Mass

$\mathrm{RA}$

Calc. Mass

$\mathrm{mDa}$

PPM

DBE

i-EIT

Formula

285.1181

100.00

285.1187

$-0.6$

$-2.1$

9.0

2.6

C17 H19 N 160 S 


\section{Elemental Composition Report}

Page 1

Multiple Mass Analysis: 2 mass(es) processed - displaying only valid results

Tolerance $=5.0$ PPM / DBE: $\min =-1.5, \max =50.0$

Element prediction: Off

Monoisotopic Mass, Odd and Even Electron Ions

155 formula(e) evaluated with 1 results within limits (all results (up to 1000) for each mass)

Elements Used:

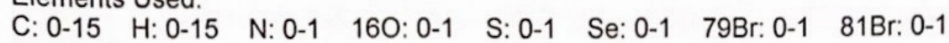

DEFAULT

Z1216- 4246 (4.100) Cm (244:251-(156:163+117:125))
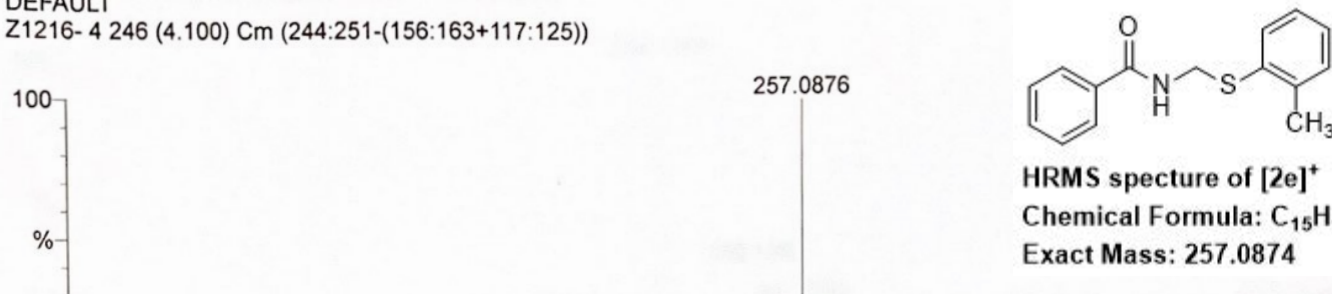

TOF MS El+

$5.40 \mathrm{e}+003$

HRMS specture of [2e $]^{+}$

Chemical Formula: $\mathrm{C}_{15} \mathrm{H}_{15} \mathrm{NOS}^{+}$

Exact Mass: 257.0874

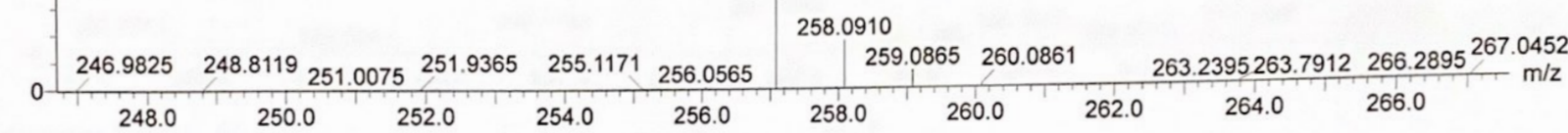

Minimum: $\quad 10.00$

Maximum: $\quad 100.00$

$30.0 \quad 5.0 \quad-1.5$

Mass

RA Calc. Mass

$\mathrm{mDa}$

PPM

DBE

i-FIT

Formula

257.0876

100.00

257.0874

0.2

0.8

9.0

0.4

C15 H15 N $160 \mathrm{~S}$ 
Multiple Mass Analysis: 2 mass(es) processed - displaying only valid results

Tolerance $=5.0$ PPM / DBE: $\min =-1.5, \max =50.0$

Element prediction: Off

Monoisotopic Mass, Odd and Even Electron Ions

33 formula(e) evaluated with 1 results within limits (all results (up to 1000) for each mass)

Elements Used:

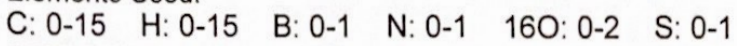

DEFAULT

1220-2 238 (3.967) Cm (236:243-(159:164+143:153))

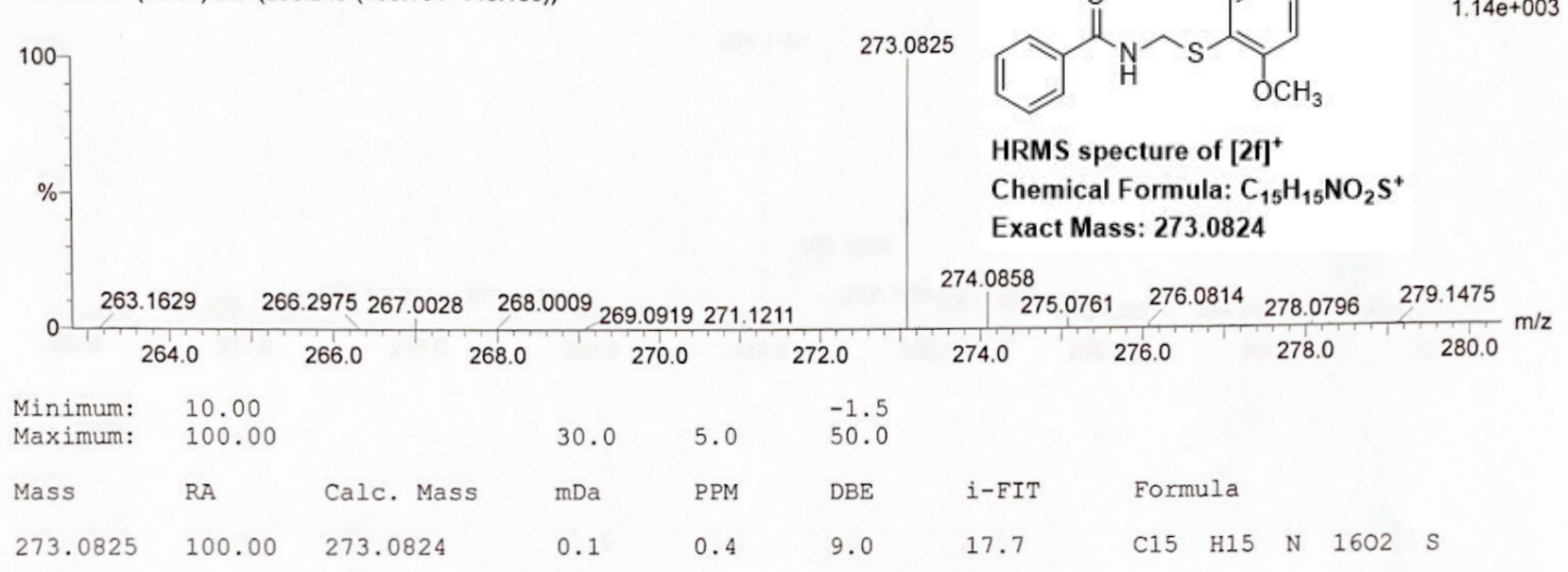


Multiple Mass Analysis: 2 mass(es) processed - displaying only valid results

Tolerance $=5.0$ PPM / DBE: $\min =-1.5, \max =50.0$

Element prediction: Off

Monoisotopic Mass, Odd and Even Electron lons

544 formula(e) evaluated with 1 results within limits (all results (up to 1000) for each mass)

Elements Used:

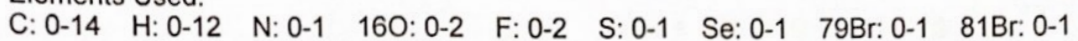

DEFAULT

Z1216-4-185 (3.083) Cm (185:189-(126:138+108:118))
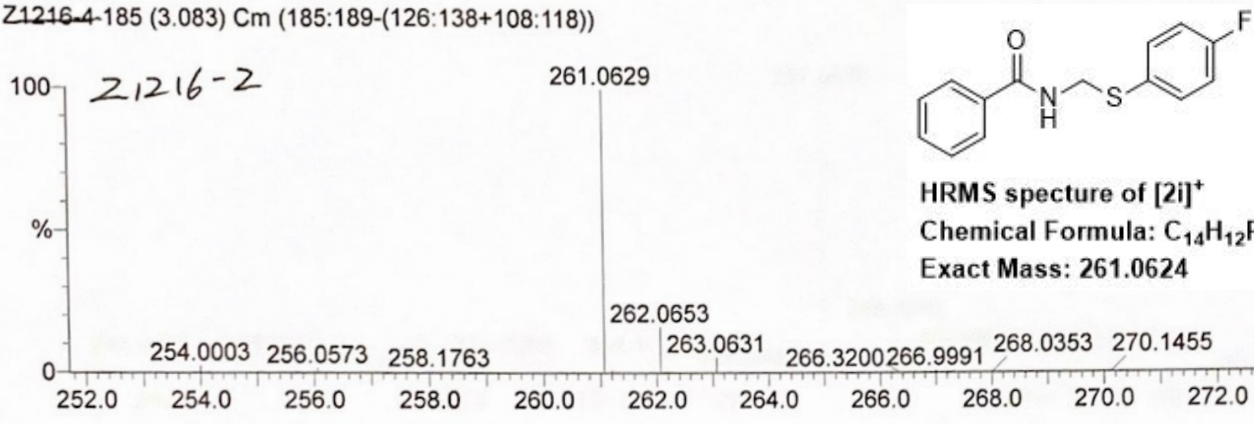

HRMS specture of [2i] ${ }^{+}$

Chemical Formula: $\mathrm{C}_{14} \mathrm{H}_{12} \mathrm{FNOS}^{+}$

Exact Mass: 261.0624

262.0653

\begin{tabular}{|lllllll}
263.0631 & 266.3200266 .9991 & 268.0353 & 270.1455 & 274.2599 & 275.2156 & 278.1448 \\
\hline
\end{tabular}

Minimum: $\quad 10.00$

Maximum: $\quad 100.00$

$\begin{array}{lll}30.0 & 5.0 & -1.5 \\ & 50.0\end{array}$

Mass RA Calc. Mass

$\mathrm{mDa}$

PPM

$\mathrm{DBE}$

i-EIT

1.9

9.0

2.9

Formula

$261.0629 \quad 100.00 \quad 261.0624$

0.5

(1)

TOF MS EI+

$4.01 e+003$

\section{S131}


Multiple Mass Analysis: 2 mass(es) processed - displaying only valid results

Tolerance $=5.0$ PPM / DBE: $\min =-1.5, \max =50.0$

Element prediction: Off

Monoisotopic Mass, Odd and Even Electron lons

105 formula(e) evaluated with 1 results within limits (all results (up to 1000) for each mass)

Elements Used:

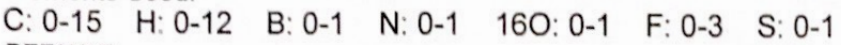

DEFAULT

$1220-4477$ (7.950) Cm (471:479-(393:404+372:380))
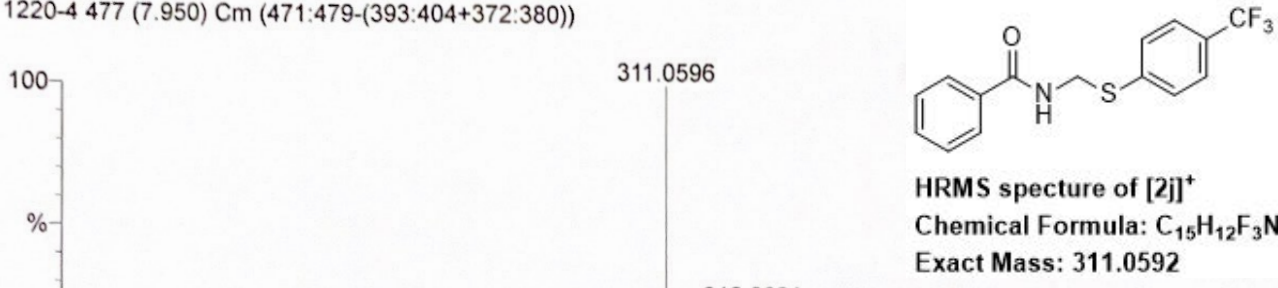

HRMS specture of $[2 \mathrm{j}]^{+}$

Chemical Formula: $\mathrm{C}_{15} \mathrm{H}_{12} \mathrm{~F}_{3} \mathrm{NOS}^{+}$

Exact Mass: 311.0592

312.0621

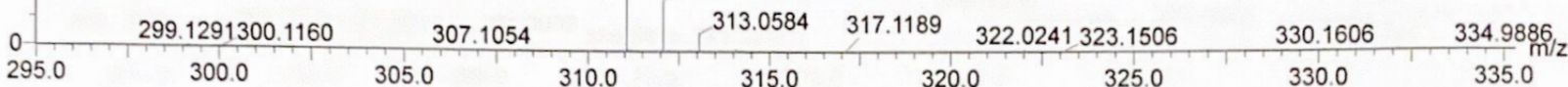

Minimum: 10.00

Maximum: 100.00

30.0

5.0

$-1.5$

Mass

RA

Calc. Mass

$\mathrm{mDa}$

PPM

$\mathrm{DBE}$

i-FIT

Formula

$311.0596 \quad 100.00$

311.0592

0.4

1.3

9.0

0.8

C15 H12 N 160 F3 S 
Multiple Mass Analysis: 4 mass(es) processed - displaying only valid results

Tolerance $=5.0$ PPM / DBE: $\min =-1.5, \max =50.0$

Element prediction: Off

Monoisotopic Mass, Odd and Even Electron lons

497 formula(e) evaluated with 1 results within limits (all results (up to 1000) for each mass)

Elements Used:

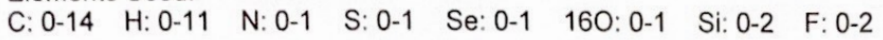

DEFAULT

Z21-329-3 842 (14.033) Cm (842:844-(797:799+806:808))

TOF MS EI+

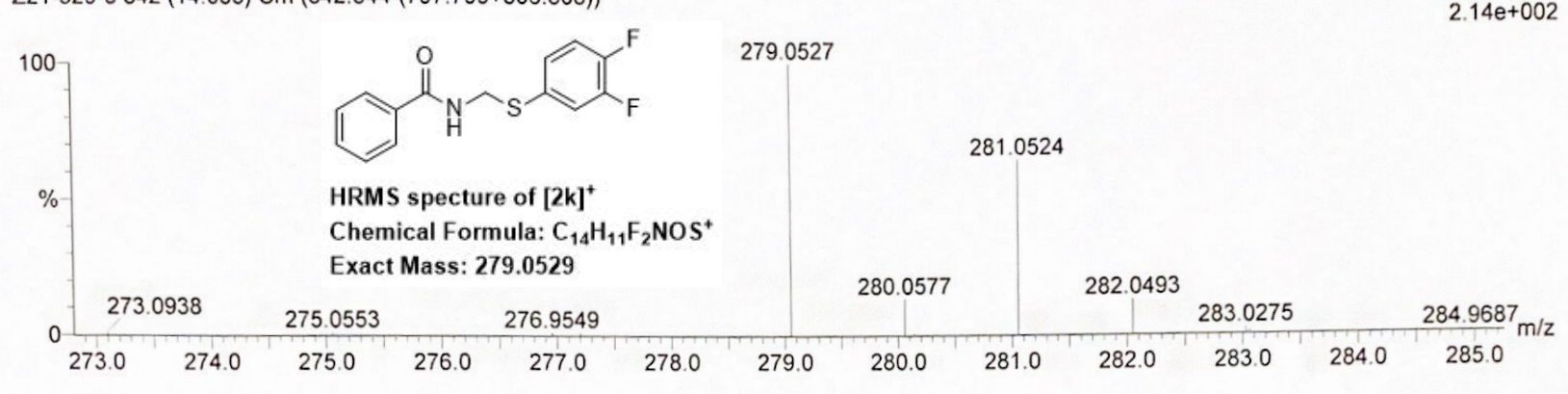

Minimum: $\quad 5.00$

Maximum: $\quad 100.00$

$\begin{array}{lll}30.0 & 5.0 & -1.5 \\ & 50.0\end{array}$

Mass RA Calc. Mass mDa PPM DBE i-FIT Formula

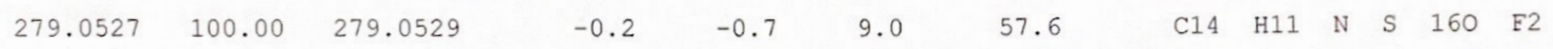


Multiple Mass Analysis: 3 mass(es) processed - displaying only valid results

Tolerance $=5.0$ PPM / DBE: $\min =-1.5, \max =50.0$

Element prediction: Off

Monoisotopic Mass, Odd and Even Electron lons

54 formula(e) evaluated with 1 results within limits (all results (up to 1000) for each mass)

Elements Used:

$\begin{array}{llllll}\text { C: } 0-12 & H: 0-12 & \text { B: } 0-1 & \text { N: }: 0-1 & \text { 160: 0-1 } & \text { S: } 0-2\end{array}$ DEFAULT

14-2 296 (4.933) Cm (296:315-(103:140+62:86))
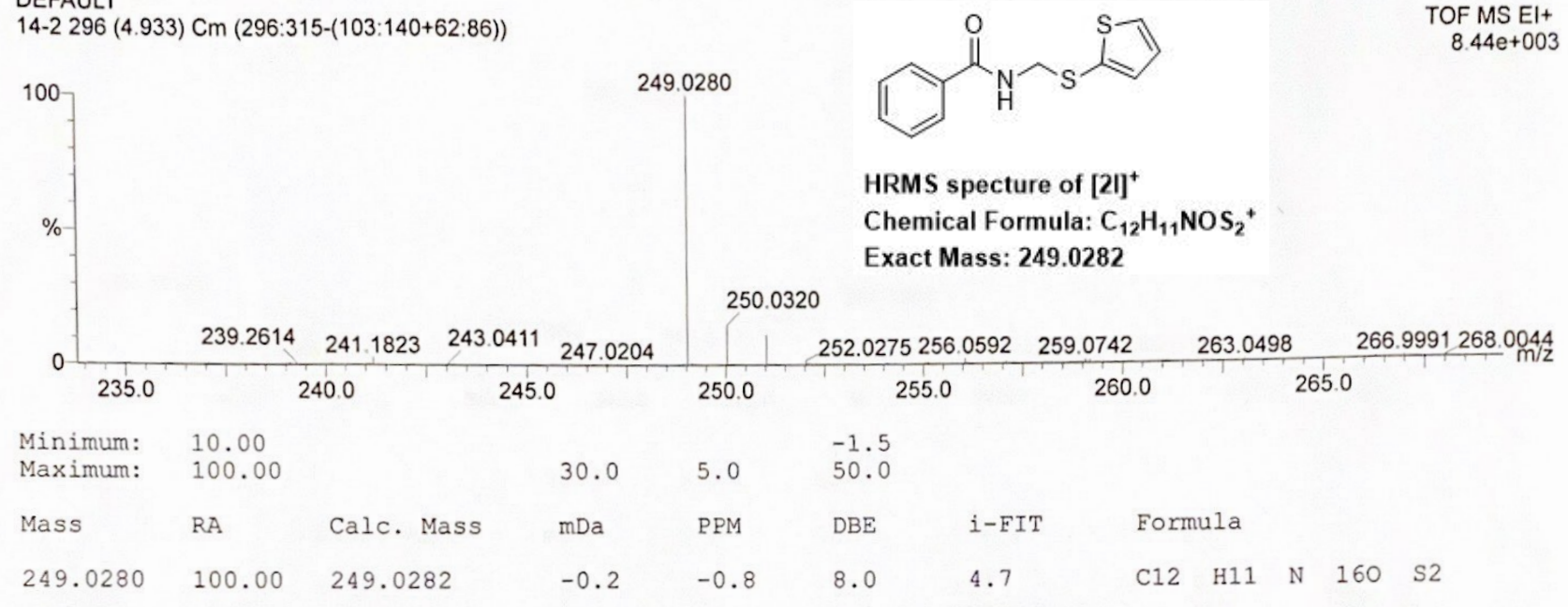

TOF MS EI+

$8.44 \mathrm{e}+003$ 
Multiple Mass Analysis: 2 mass(es) processed - displaying only valid results

Tolerance $=5.0$ PPM / DBE: $\min =-1.5, \max =50.0$

Element prediction: Off

Monoisotopic Mass, Odd and Even Electron lons

146 formula(e) evaluated with 1 results within limits (all results (up to 1000) for each mass)

Elements Used

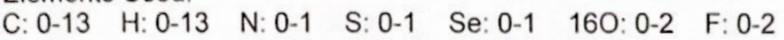

DEFAULT

Z21-44-3 144 (2.400) Cm (144:148)

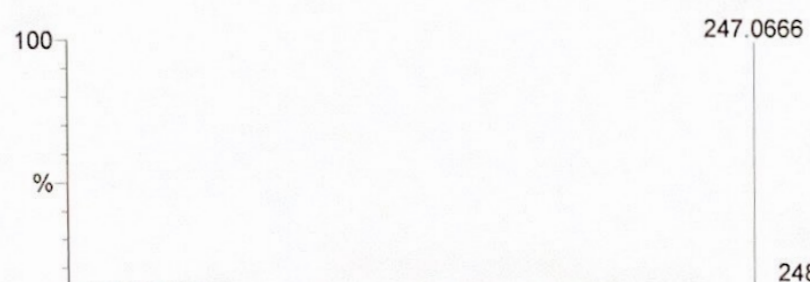

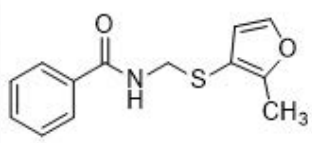

HRMS specture of $[2 \mathrm{~m}]^{+}$

Chemical Formula: $\mathrm{C}_{13} \mathrm{H}_{13} \mathrm{NO}_{2} \mathrm{~S}^{+}$

Exact Mass: 247.0667

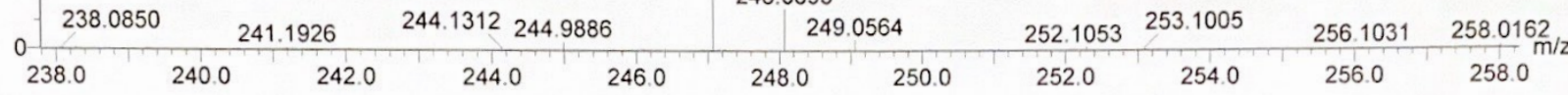

Minimum: 5.00

Maximum: 100.00

$\begin{array}{lll}30.0 & 5.0 & -1.5 \\ & 50.0\end{array}$

RA

Calc. Mass

$\mathrm{mDa}$

PPM

DBE

$-0.1$

$-0.4$

8.0

i-FIT

Formula

247.0666

$100.00 \quad 247.0667$

-

6.5

$\begin{array}{lllll}\mathrm{C} 13 & \mathrm{H} 13 & \mathrm{~N} & \mathrm{~S} & 1602\end{array}$ 
Multiple Mass Analysis: 3 mass(es) processed - displaying only valid results

Tolerance $=5.0$ PPM / DBE: $\min =-1.5, \max =50.0$

Element prediction: Off

Monoisotopic Mass, Odd and Even Electron lons

16 formula(e) evaluated with 1 results within limits (all results (up to 1000) for each mass)

Elements Used:

$\begin{array}{lllll}\text { C: } 0-20 & H: 0-33 & \text { N: } 0-1 & 160: 0-1 & \text { S: } 0-1\end{array}$

DEFAULT

Z21-430 594 (9.900) Cm (587:594-(494:504+470:479))

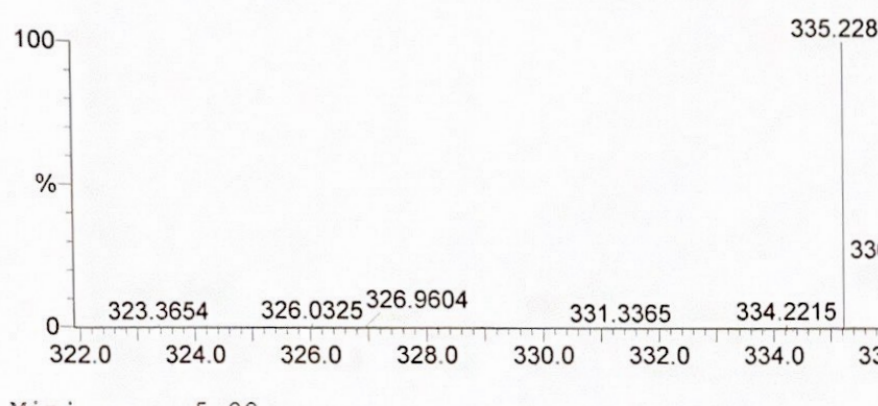

35.2287

Minimum: $\quad 5.00$

Maximum: $\quad 100.00$

30.0

5.0

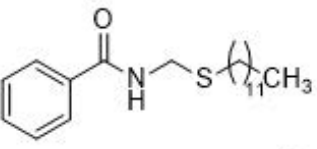

HRMS specture of $[2 \mathrm{n}]^{+}$

Chemical Formula: $\mathrm{C}_{20} \mathrm{H}_{33} \mathrm{NOS}^{+}$

Exact Mass: 335.2283

336.2322

Mass

RA Calc. Mass

$\mathrm{mDa}$

PPM

0.4

1.2

$335.2287 \quad 100.00 \quad 335.2283$
i-EIT

Formula

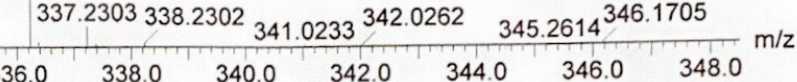

$-1.5$

DBE

2.0

$\mathrm{C} 20 \mathrm{H} 33 \mathrm{~N} 160 \mathrm{~S}$
TOF MS El+

$6.80 \mathrm{e}+003$ 


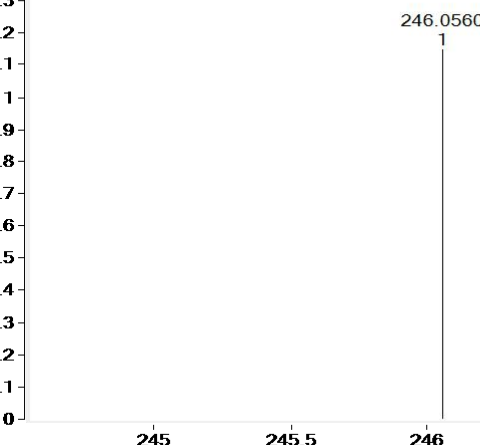<smiles>CC(=O)NCC(=O)Sc1ccc(C)cc1</smiles>

HRMS specture of [3a+Na] $]^{+}$

Chemical Formula: $\mathrm{C}_{11} \mathrm{H}_{13} \mathrm{NO}_{2} \mathrm{SNa}^{+}$

Exact Mass: 246.0559

$\mathbf{x 1 0} 4$ +ESI Scan (1.6171333333 min) Frag=100.0V Z5.d

245.5

$246 \quad 246.5$
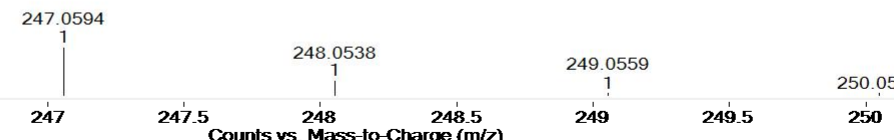

250.5

251

251.5

2.4<smiles>COC(=O)NCC(=O)Sc1ccc(C)cc1</smiles>

HRMS specture of $[3 b+\mathrm{H}]^{+}$

Chemical Formula: $\mathrm{C}_{11} \mathrm{H}_{14} \mathrm{NO}_{3} \mathrm{~S}^{+}$

Exact Mass: 240.0689

$\mathbf{x 1 0}^{\mathbf{5}}$ +ESI Scan (2.4157500000 $\left.\mathrm{min}\right)$ Frag=100.0V Z6-2.d

6
5
4
3
2
1
0

65

304.0980



HRMS specture of [3c+Na] ${ }^{+}$

Chemical Formula: $\mathrm{C}_{14} \mathrm{H}_{19} \mathrm{NO}_{3} \mathrm{SNa}^{+}$

Exact Mass: 304.0978
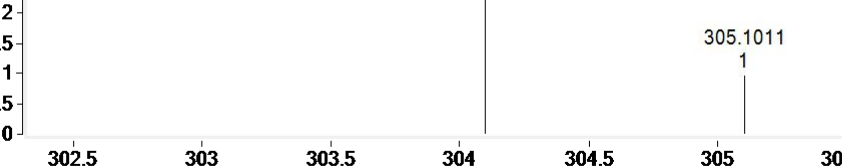

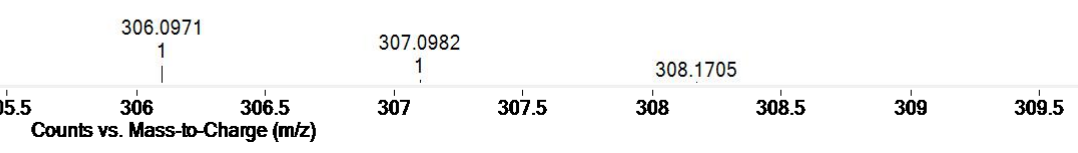

$\times 10^{4}$
8.5
8
7.5
7
6.5
6
5.5
5
4.5
4
3.5
3
2.5
2
1.5
1
0.5
0

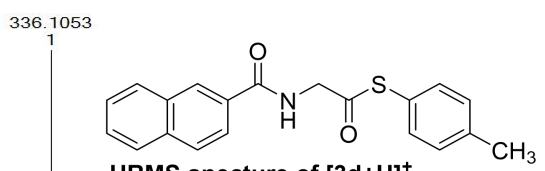

HRMS specture of $[3 \mathrm{~d}+\mathrm{H}]^{+}$

Chemical Formula: $\mathrm{C}_{20} \mathrm{H}_{18} \mathrm{NO}_{2} \mathrm{~S}^{+}$

Exact Mass: 336.1053

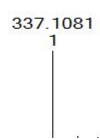

338.1048

339.1065

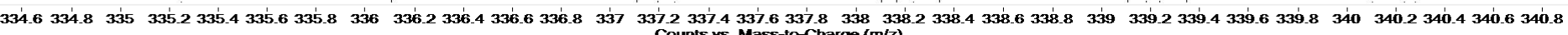



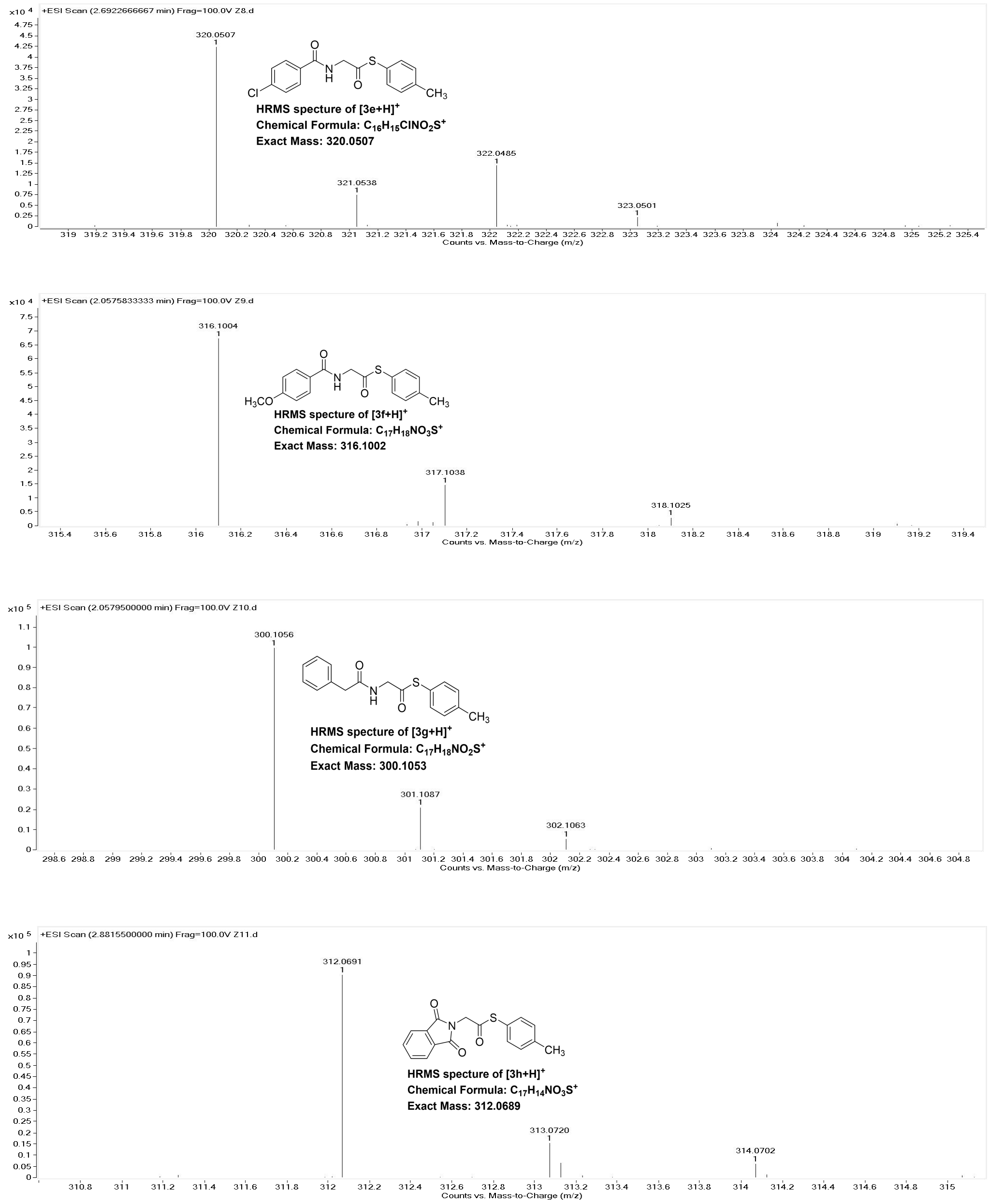

S138 


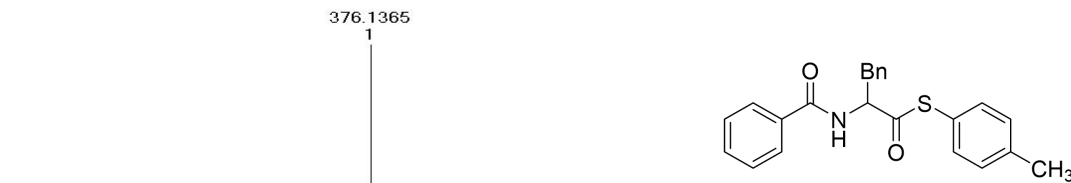

HRMS specture of $[3 \mathbf{i}+\mathrm{H}]^{+}$

Chemical Formula: $\mathrm{C}_{23} \mathrm{H}_{22} \mathrm{NO}_{2} \mathrm{~S}^{+}$

Exact Mass: 376.1366
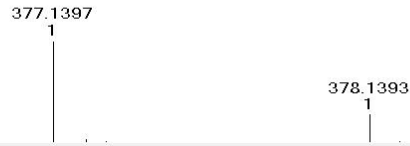

379.1373

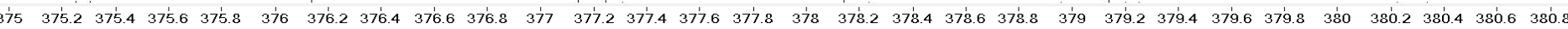
Counts vs. Mass to-Charge $(\mathrm{m} / \mathrm{z})$

$\times 10^{4}+$ ESI Scan (3.5421000000 min) Frag $=100.0 \mathrm{~V} Z 13 . \mathrm{d}$

$\times 10$
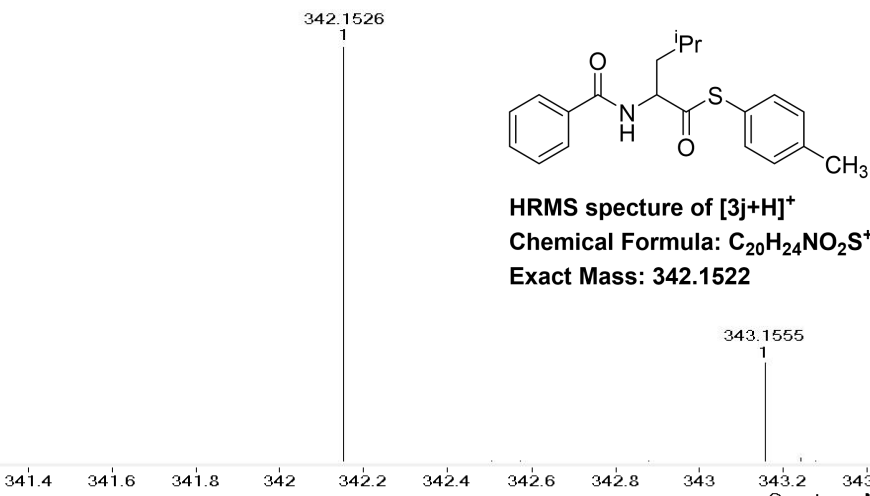

HRMS specture of $[3 \mathrm{j}+\mathrm{H}]^{+}$

Chemical Formula: $\mathrm{C}_{20} \mathrm{H}_{24} \mathrm{NO}_{2} \mathrm{~S}^{+}$

Exact Mass: $\mathbf{3 4 2 . 1 5 2 2}$

$\begin{array}{lll}34.4 & 341.8 \quad 342\end{array}$
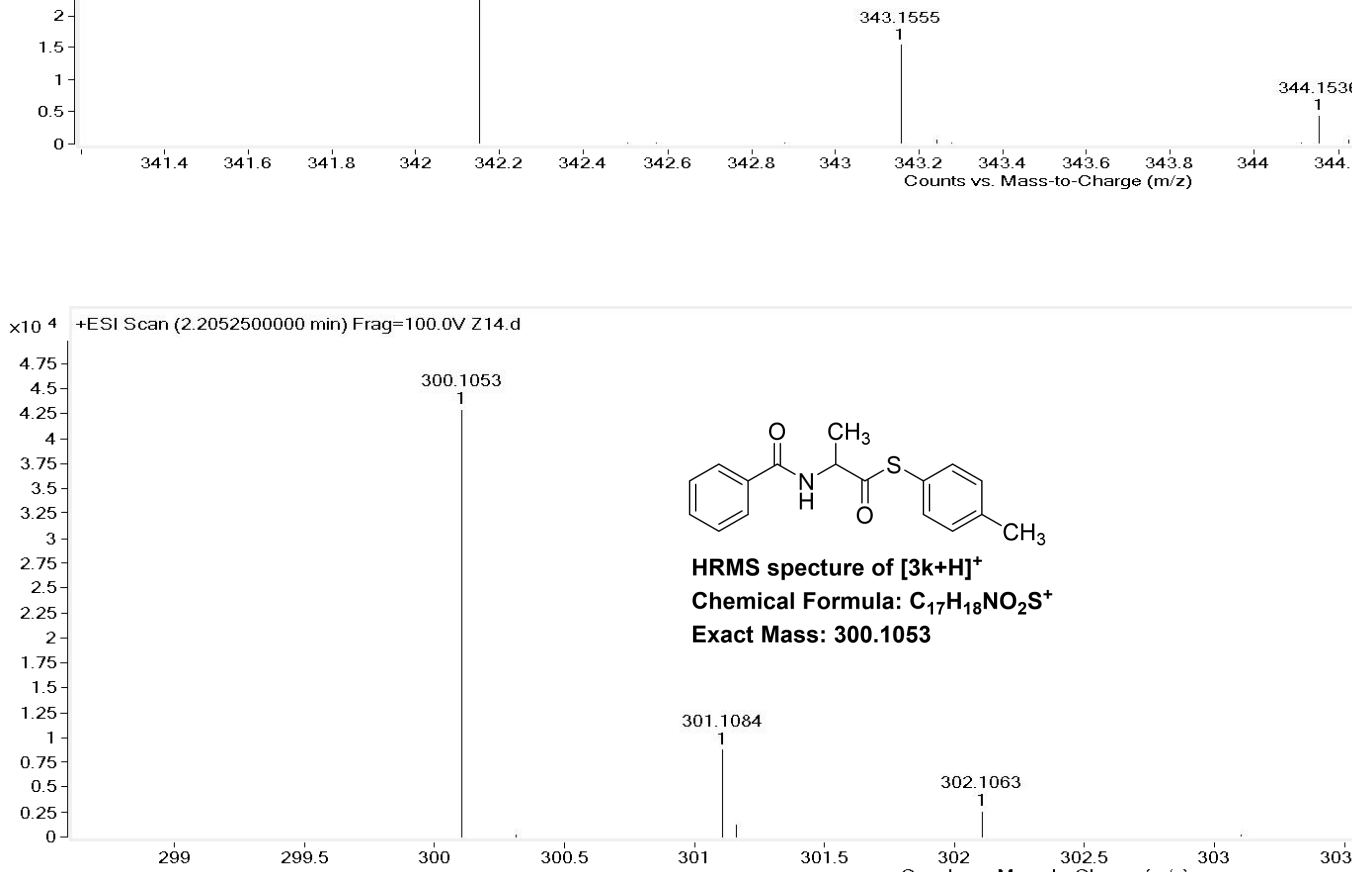

HRMS specture of [3k+H]

Chemical Formula: $\mathrm{C}_{17} \mathrm{H}_{18} \mathrm{NO}_{2} \mathrm{~S}^{+}$

Exact Mass: $\mathbf{3 0 0 . 1 0 5 3}$
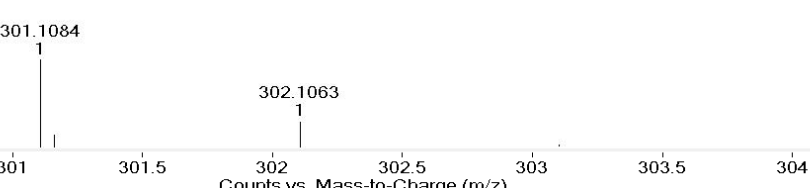

304.5

305

6 +ESI Scan (2.4533000000 min) Frag=100.0V Z15-2.d
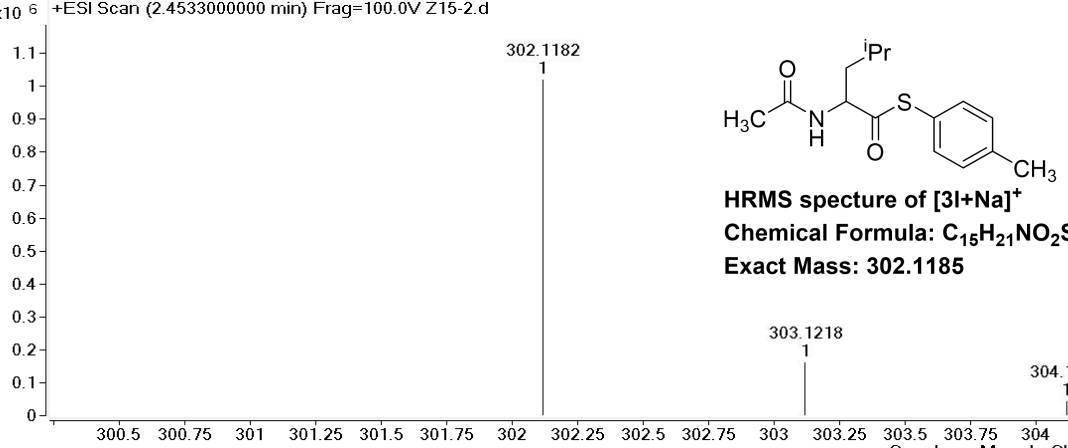

HRMS specture of $[31+\mathrm{Na}]^{+}$

Chemical Formula: $\mathrm{C}_{15} \mathrm{H}_{21} \mathrm{NO}_{2} \mathrm{SNa}^{+}$

Exact Mass: $\mathbf{3 0 2 . 1 1 8 5}$

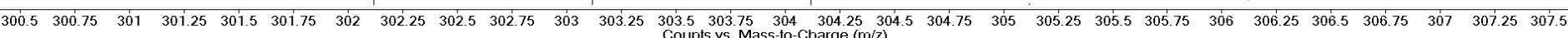

\section{S139}


Multiple Mass Analysis: $\mathbf{4}$ mass(es) processed - displaying only valid results

Tolerance $=5.0$ PPM / DBE: $\min =-1.5, \max =50.0$

Element prediction: Off

Monoisotopic Mass, Odd and Even Electron lons

97 formula(e) evaluated with 1 results within limits (all results (up to 1000) for each mass)

Elements Used:

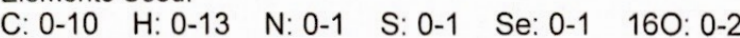
DEFAULT

Z21-330-1 152 (2.533) Cm (152:154-(112:117+96:102))

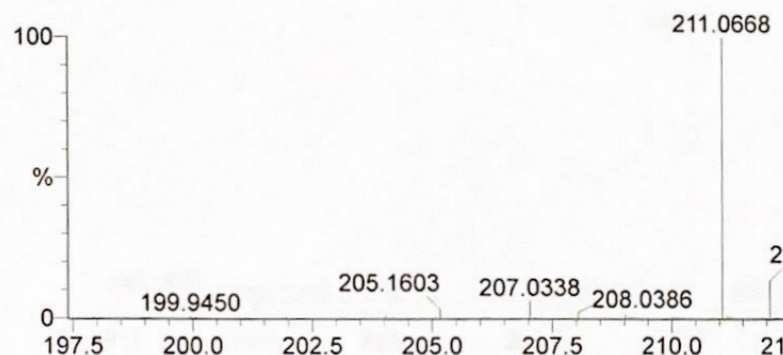

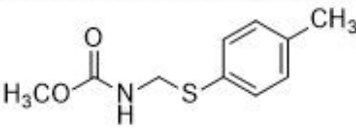

HRMS specture of $[4 b]^{+}$

Chemical Formula: $\mathrm{C}_{10} \mathrm{H}_{13} \mathrm{NO}_{2} \mathrm{~S}^{+}$

Exact Mass: 211.0667
$5.19 e+003$
Minimum: $\quad 5.00$

Maximum: 100.00

Mass

211.0668

RA

Calc. Mass

30.0

$\mathrm{mDa}$

0.1
100.00

211.0667

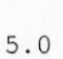

PPM

0.5

212.0701

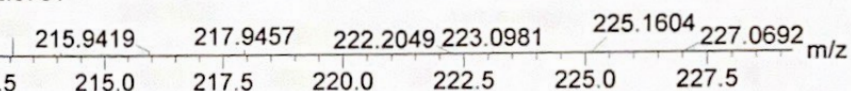

$-1.5$

50.0

DBE

i-EIT

Formula

5.0

2.5

$\begin{array}{llll}\mathrm{C} 10 \mathrm{H} 13 \mathrm{~N} & \mathrm{~S} & 1602\end{array}$ 
Multiple Mass Analysis: 3 mass(es) processed - displaying only valid results

Tolerance $=5.0$ PPM / DBE: $\min =-1.5, \max =50.0$

Element prediction: Off

Monoisotopic Mass, Odd and Even Electron Ions

89 formula(e) evaluated with 1 results within limits (all results (up to 1000) for each mass)

Elements Used:

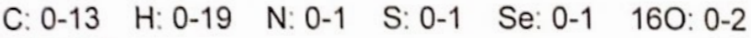

DEFAULT

Z21-329-2 $142(2.367) \mathrm{Cm}(142: 145-(73: 79+61: 69))$

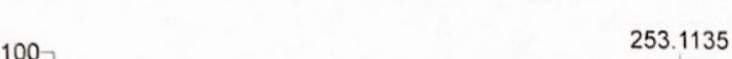

100

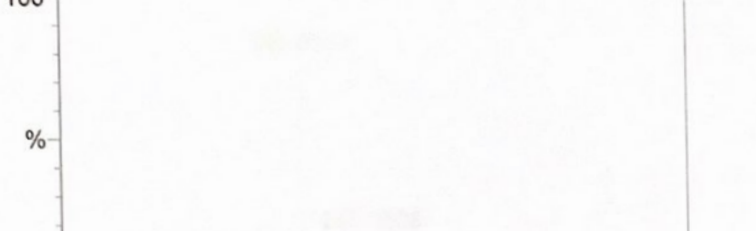

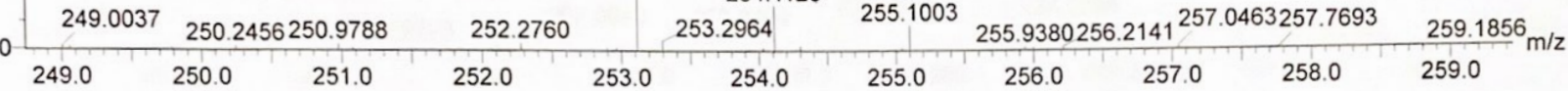

Minimum: $\quad 5.00$

Maximum: $\quad 100.00$

$30.0 \quad 5.0 \quad-1.5$

Mass

RA Calc. Mass

$\mathrm{mDa}$

PPM

50.0

$\begin{array}{lll}253.1135 & 100.00 \quad 253.1137\end{array}$

$\begin{array}{ll}-0.2 & -0.8\end{array}$

DBE

i-FIT

Formula

253.1135 .100 .000 .253 .1137

1.1

C13 H19 N S 1602

TOF MS El+

$2.59 \mathrm{e}+002$ 
Multiple Mass Analysis: 3 mass(es) processed - displaying only valid results

Tolerance $=5.0$ PPM / DBE: $\min =-1.5, \max =50.0$

Element prediction: Off

Monoisotopic Mass, Odd and Even Electron lons

12 formula(e) evaluated with 1 results within limits (all results (up to 1000) for each mass)

Elements Used:

$\begin{array}{lllll}\text { C: } 0-19 & H: 0-23 & \text { N: }: 0-1 & \text { 160: } 0-1 & \text { S: } 0-1\end{array}$

DEFAULT

Z21-420 224 (3.733) Cm (217:224)
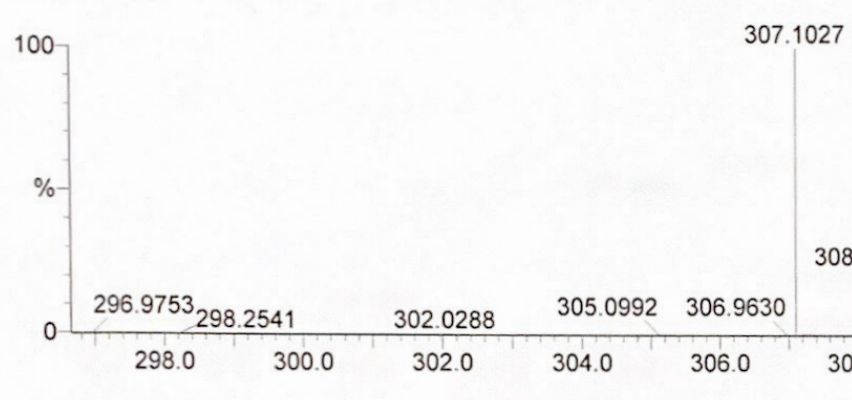

Minimum: $\quad 5.00$

Maximum: 100.00

$\begin{array}{lll}30.0 & 5.0 & -1.5 \\ 30.0\end{array}$

Mass

RA Calc. Mass

$\mathrm{mDa}$

PPM

$\mathrm{DBE}$

i-EIT

Eormula

$307.1027 \quad 100.00 \quad 307.1031$

$-0.4-1.3$

12.0

0.7

$\begin{array}{lllll}\mathrm{C} 19 & \mathrm{H} 17 & \mathrm{~N} & 160 \mathrm{~S}\end{array}$

TOF MS EI+

$1.26 e+004$

HRMS specture of $[4 \mathrm{~d}]^{+}$

Chemical Formula: $\mathrm{C}_{19} \mathrm{H}_{19} 7 \mathrm{OS}$

Exact Mass: 307.1031

308.1067

$310.1036 \quad 311.8145313 .9836 \quad 316.1989 \quad 317.3250$

$\begin{array}{llllll}310.0 & 312.0 & 314.0 & 316.0 & 318.0\end{array}$


Multiple Mass Analysis: 2 mass(es) processed - displaying only valid results

Tolerance $=5.0$ PPM / DBE: $\min =-1.5, \max =50.0$

Element prediction: Off

Monoisotopic Mass, Odd and Even Electron Ions

62 formula(e) evaluated with 1 results within limits (all results (up to 1000) for each mass)

Elements Used:

$\begin{array}{llllll}\text { C: } 0-16 & H: 0-19 & \text { N: }: 0-1 & \text { S: 0-1 } & \text { Se: 0-1 } & \text { 160: 0-2 }\end{array}$

DEFAULT

Z21-44-4 189 (3.150) Cm (189:194-(75:80+63:70))

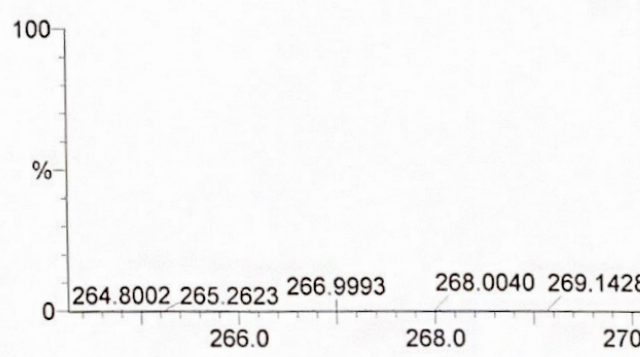

\subsection{4}

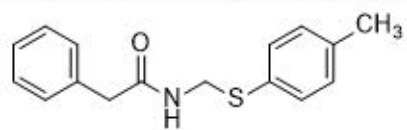

TOF MS EI+

$2.12 \mathrm{e}+003$

HRMS specture of $[4 \mathrm{~g}]^{+}$

Chemical Formula: $\mathrm{C}_{16} \mathrm{H}_{17} \mathrm{NOS}^{+}$

Exact Mass: 271.1031

272.1061

\section{$273.1009 \quad 274.1033275 .1266 \quad 276.2783$}

$272.0 \quad 274.0 \quad 276.0 \quad 278.0$

Minimum: $\quad 5.00$

Maximum: 100.00

$-1.5$

Mass

30.0

50.0

$\begin{array}{lllll}\text { Mass } & \text { RA } & \text { Calc. Mass } & \text { PDa }\end{array}$

$271.1034 \quad 100.00 \quad 271.1031$

$0.3 \quad 1.1$

9.0

i-EIT

Formula

0.3

1.1

4.0

$\begin{array}{lllll}\text { C16 H17 N } & \text { S } & 160\end{array}$ 
Multiple Mass Analysis: 7 mass(es) processed - displaying only valid results

Tolerance $=5.0$ PPM / DBE: $\min =-1.5, \max =50.0$

Element prediction: Off

Monoisotopic Mass, Odd and Even Electron Ions

140 formula(e) evaluated with 2 resuits within limits (all results (up to 1000) for each mass)

Elements Used:

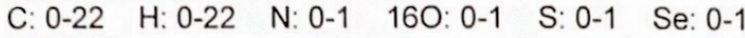

DEFAULT

Z21-22-2 174 (2.900) Cm (174:177-(82:87+59:63))

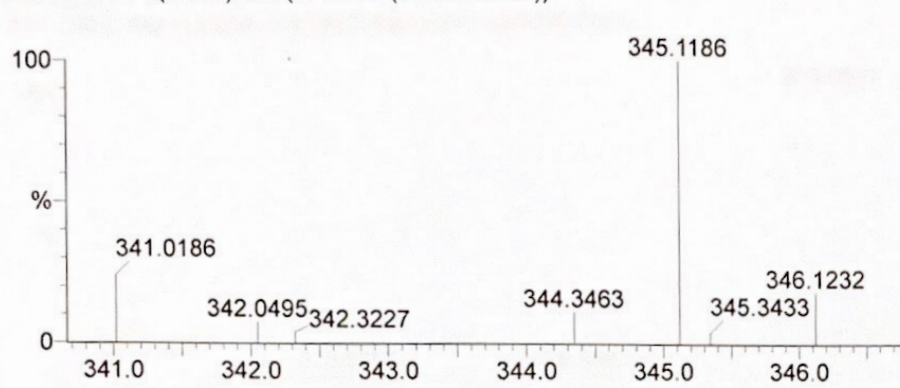

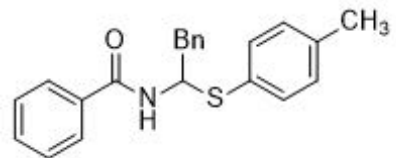

HRMS specture of [4i] ${ }^{+}$

Chemical Formula: $\mathrm{C}_{22} \mathrm{H}_{21} \mathrm{NOS}^{+}$

Exact Mass: 347.1344

347.1351
Minimum: $\quad 5.00$

Maximum: 100.00

Mass RA Calc. Mass

$\begin{array}{lll}345.1186 & 100.00 & 345.1187\end{array}$

$\begin{array}{lll}347.1351 & 23.63 & 347.1344\end{array}$

$\begin{array}{lll}30.0 & 5.0 & -1.5 \\ & & 50.0\end{array}$

$\mathrm{mDa} \quad \mathrm{PPM}$

DBE

i-FIT Formula

$\begin{array}{lll}-0.1 & -0.3 & 14.0\end{array}$

2.0
TOF MS EI+

$5.57 \mathrm{e}+001$ 
Multiple Mass Analysis: 3 mass(es) processed - displaying only valid results

Tolerance $=5.0$ PPM / DBE: $\min =-1.5, \max =50.0$

Element prediction: Off

Monoisotopic Mass, Odd and Even Electron Ions

17 formula(e) evaluated with 1 results within limits (all results (up to 1000) for each mass)

Elements Used

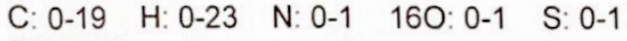

DEFAULT

Z21-54-1-20210512 $284(4.733) \mathrm{Cm}(284: 288-(222: 223+212: 214))$

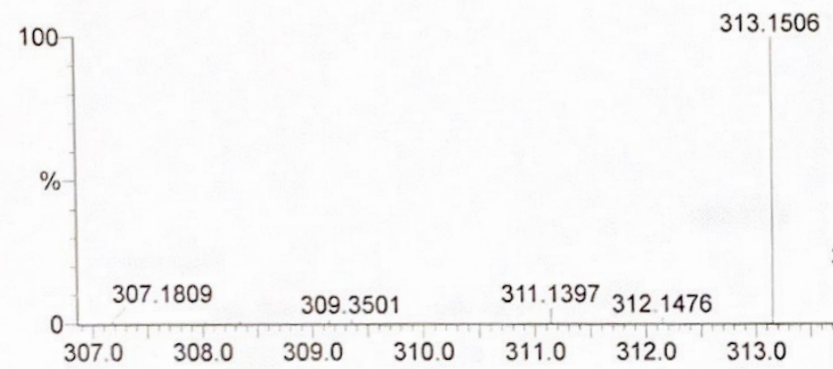

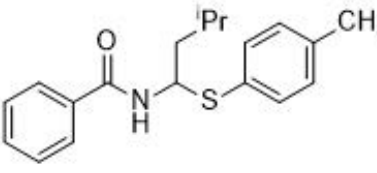

HRMS specture of $[4]^{+}$

Chemical Formula: $\mathrm{C}_{19} \mathrm{H}_{23} \mathrm{NOS}^{+}$

Exact Mass: 313.1500

314.1483

Minimum: $\quad 5.00 \quad-1.5$

Minimum

Maximum: $\quad 100.00$

30.0

5.0

50.0

Mass

RA Calc. Mass

$\mathrm{mDa}$

PPM

DBE

i-EIT

Formula

313.1506

$100.00 \quad 313.1500$

0.6

1.9

9.0

40.8

C19 H23 N $160 \mathrm{~S}$ 
Multiple Mass Analysis: 3 mass(es) processed - displaying only valid results

Tolerance $=5.0$ PPM / DBE: $\min =-1.5, \max =50.0$

Element prediction: Off

Monoisotopic Mass, Odd and Even Electron lons

257 formula(e) evaluated with 1 results within limits (all results (up to 1000) for each mass)

Elements Used:

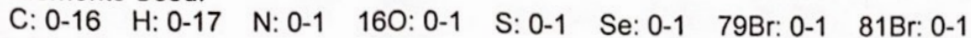

DEFAULT

927-3 181 (3.020) Cm (180:182-(150:155+141:145))

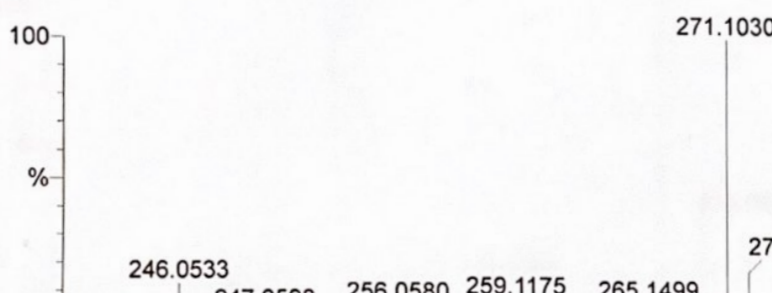

030

$\begin{array}{lll}259.1175 & 265.1499\end{array}$

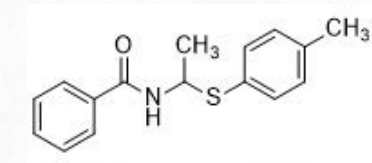

HRMS specture of [4k] ${ }^{+}$

Chemical Formula: $\mathrm{C}_{16} \mathrm{H}_{17} \mathrm{NOS}^{+}$

Exact Mass: 271.1031

$$
0
$$
250.0 260.0

$\frac{265.1499}{270.0}$

273.1037

$\frac{281.04}{280.0}$

\title{
30.0
}

5.0

$-1.5$

$\begin{array}{ll}\text { Minimum: } & 10.00 \\ \text { Maximum: } & 100.00\end{array}$

Mass

RA

Calc. Mass

$\mathrm{mDa}$

$-0.1$

\begin{abstract}
PPM
\end{abstract}
DBE

271.1030

100.00

271.1031

$-0.4$

9.0

i-FIT

Eormula

8.0

C16 H17 N $160 \mathrm{~S}$ 
Multiple Mass Analysis: 8 mass(es) processed - displaying only valid results

Tolerance $=5.0$ PPM / DBE: $\min =-1.5, \max =50.0$

Element prediction: Off

Monoisotopic Mass, Odd and Even Electron Ions

262 formula(e) evaluated with 1 results within limits (all results (up to 1000) for each mass)

Elements Used:

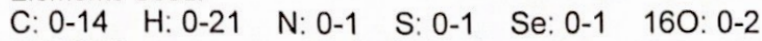

DEFAULT

Z21-331-1 171 (2.851) Cm (171:181-(135:138+129:131))
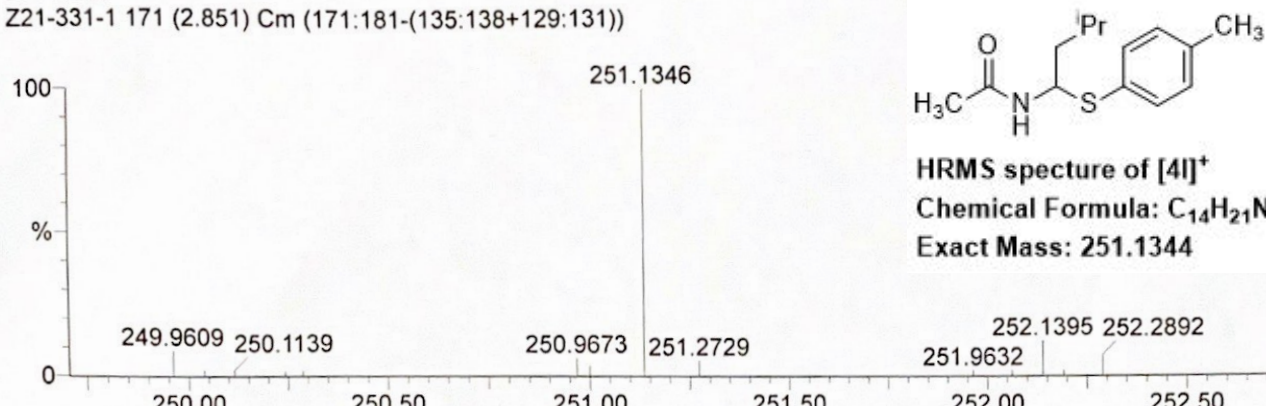

TOF MS EI+

1.17e+002

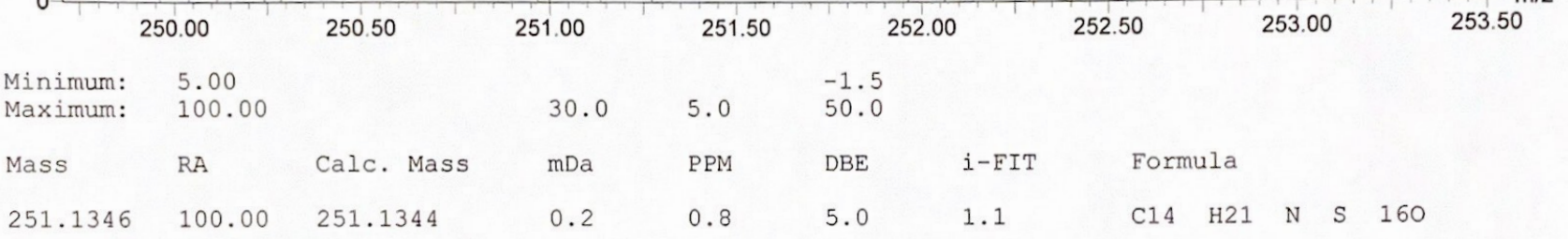


Multiple Mass Analysis: 9 mass(es) processed - displaying only valid results

Tolerance $=5.0$ PPM / DBE: $\min =-1.5, \max =50.0$

Element prediction: Off

Monoisotopic Mass, Odd and Even Electron Ions

68 formula(e) evaluated with 1 results within limits (all results (up to 1000) for each mass)

Elements Used:

$\begin{array}{lllll}\text { C: } 0-18 & \text { H: } 0-17 & \text { N: } 0-1 & \text { 160: 0-2 } & \text { S: } 0-1\end{array}$

DEFAULT

Z21-421-1 329 (5.486) Cm (329:338-(240:250+138:153))

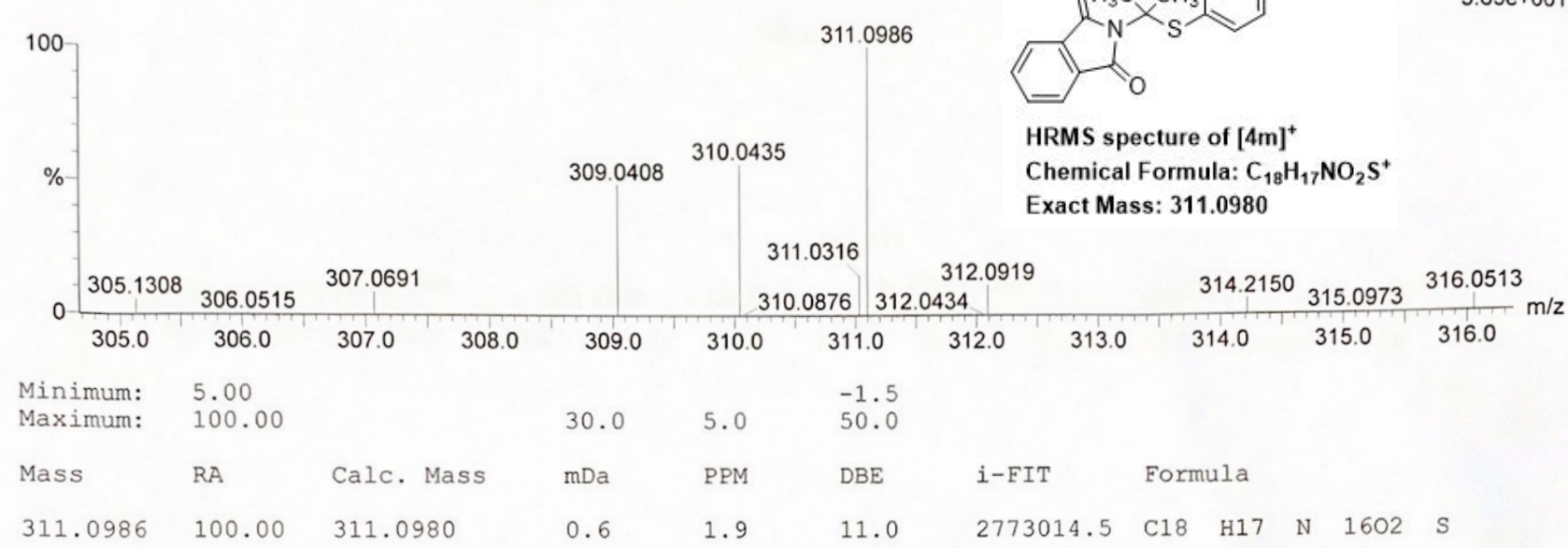




\section{Chiral HPLC of S-(p-tolyl) 2-benzamidopropanethioate (rac-3k)}<smiles>Cc1ccc(SC(=O)C(C)NC(=O)c2ccccc2)cc1</smiles>

rac-3k

HPLC: Chiralpak IG-3 column; $\lambda=254 \mathrm{~nm}$; hexane/isopropanol $=85 / 15$; flow rate $=$ $1.0 \mathrm{~mL} / \mathrm{min}$.

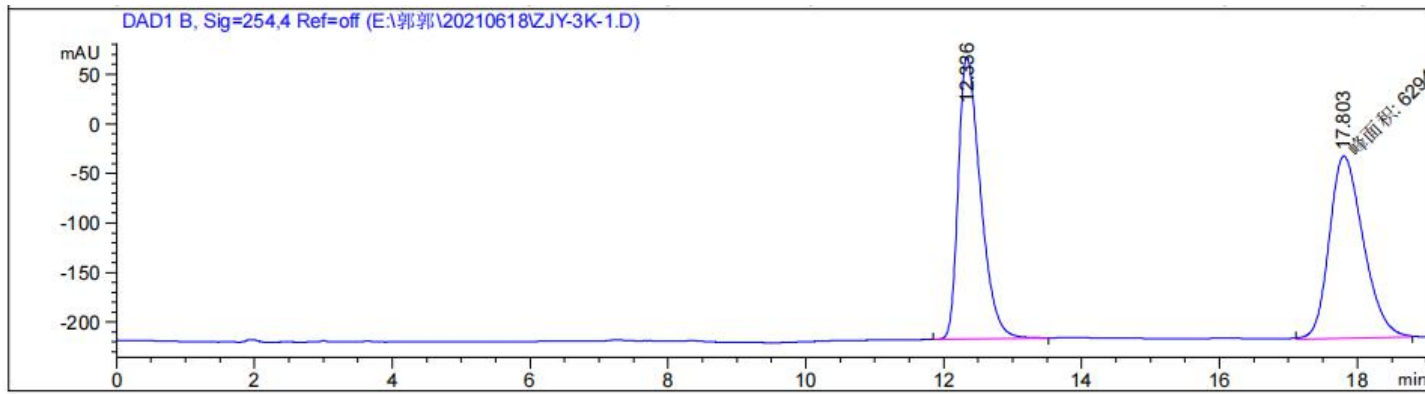

\begin{tabular}{|c|c|c|c|c|c|c|}
\hline $\begin{array}{r}\text { 峰 } \\
\text { \# }\end{array}$ & $\begin{array}{c}\text { 保留时间 } \\
\text { [min] }\end{array}$ & 类型 & $\begin{array}{l}\text { 峰宽 } \\
\text { [min] }\end{array}$ & $\begin{array}{l}\text { 峰面积 } \\
{\left[\mathrm{mAU}^{\star} \mathrm{s}\right]}\end{array}$ & $\begin{array}{l}\text { 峰高 } \\
\text { [mAU] }\end{array}$ & $\begin{array}{c}\text { 峰面积 } \\
\%\end{array}$ \\
\hline & & & & & & \\
\hline 1 & & & & 634 & 01 & \\
\hline 2 & 803 & IM & 0.5704 & 6294.81738 & 183.93155 & 49 \\
\hline
\end{tabular}

Chiral HPLC of (L)-S-(p-tolyl) 2-benzamidopropanethioate (L-3k)<smiles>Cc1ccc(SC(=O)C(C)NC(=O)c2ccccc2)cc1</smiles>

\section{L-3k}

HPLC: Chiralpak IG-3 column; $\lambda=254 \mathrm{~nm}$; hexane/isopropanol $=85 / 15$; flow rate $=$ $1.0 \mathrm{~mL} / \mathrm{min}$.

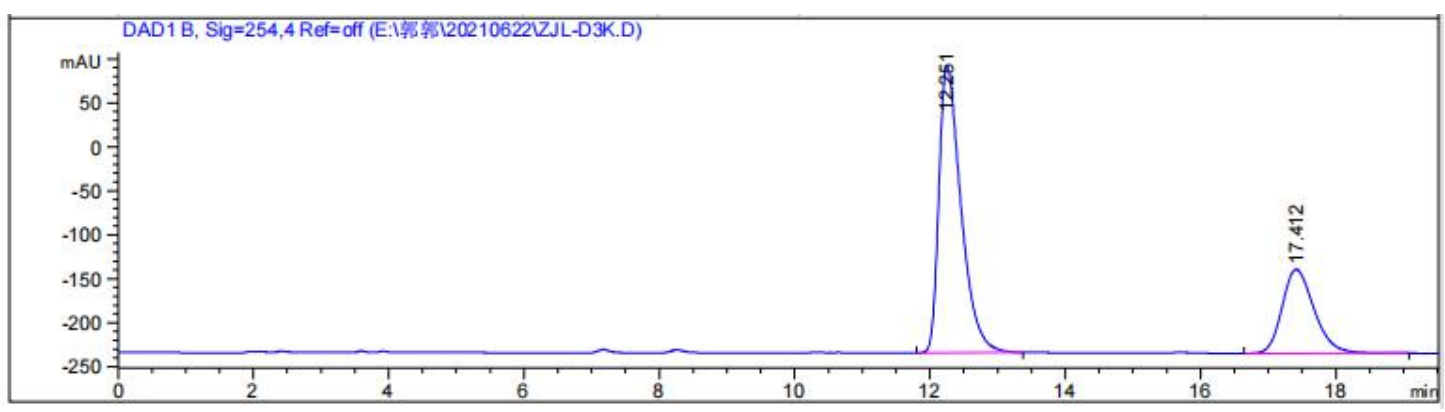

\begin{tabular}{|c|c|c|c|c|c|c|}
\hline 峰 & $\begin{array}{c}\text { 保留时间 } \\
\text { [min] }\end{array}$ & 头型 & $\begin{array}{l}\text { 峰宽 } \\
\text { [min] }\end{array}$ & $\begin{array}{l}\text { 峰面积 } \\
\text { [mAU*s] }\end{array}$ & $\begin{array}{l}\text { 峰高 } \\
\text { [mAU] }\end{array}$ & $\begin{array}{c}\text { 峰面积 } \\
\text { 各 }\end{array}$ \\
\hline- & ------ & & -- & --------- & $-\infty$ & --- \\
\hline 1 & 12.251 & BB & 0.3447 & 7381.78564 & 325.38620 & 70.2086 \\
\hline 2 & 17.412 & BB & 0.5052 & 3132.29395 & 95.35493 & 29.7914 \\
\hline
\end{tabular}




\section{Chiral HPLC of N-(1-(p-tolylthio)ethyl)benzamide (rac-4k)}<smiles>Cc1ccc(SC(C)NC(=O)c2ccccc2)cc1</smiles>

HPLC: Chiralpak IG-3 column; $\lambda=254 \mathrm{~nm}$; hexane/isopropanol $=85 / 15$; flow rate $=$ $1.0 \mathrm{~mL} / \mathrm{min}$.
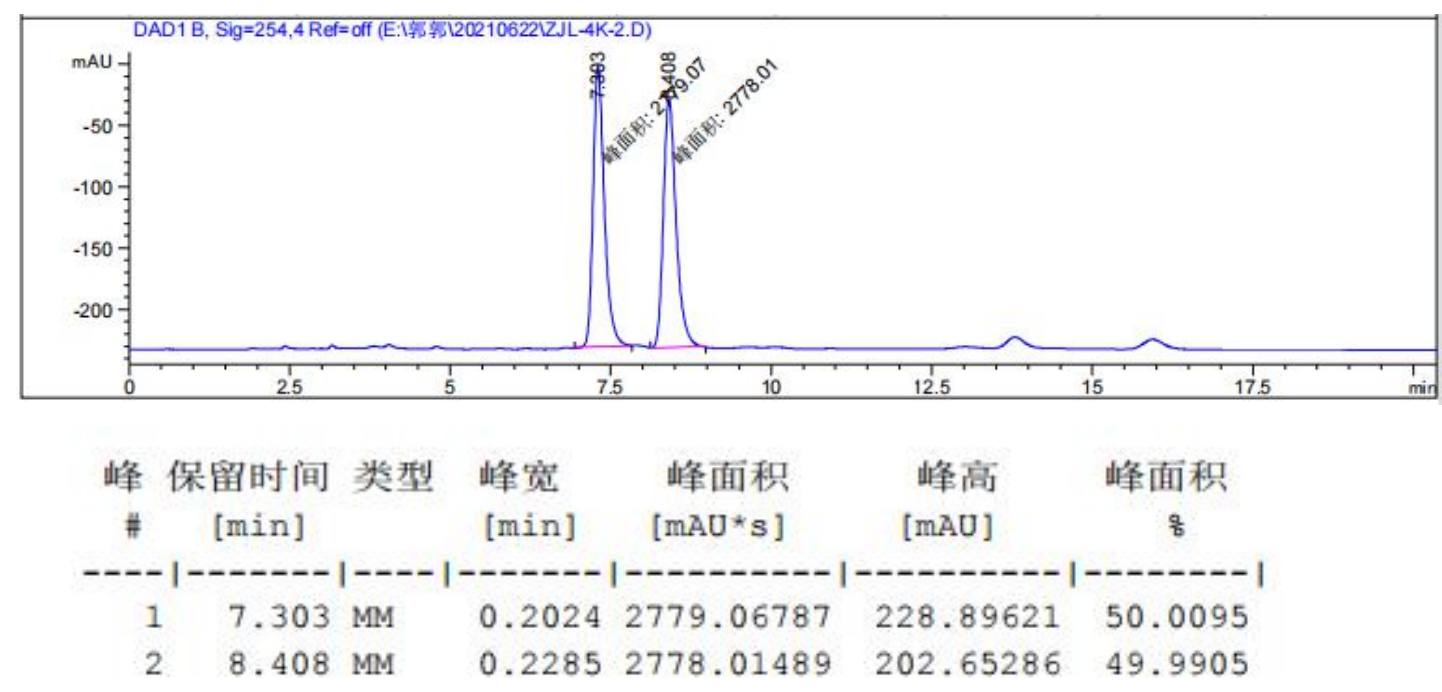

\section{Chiral HPLC of N-(1-(p-tolylthio)ethyl)benzamide (D/L-4k)}<smiles>Cc1ccc(SC(C)NC(=O)c2ccccc2)cc1</smiles>

\section{D/L-4k}

HPLC: Chiralpak IG-3 column; $\lambda=254 \mathrm{~nm}$; hexane/isopropanol =85/15; flow rate $=$ $1.0 \mathrm{~mL} / \mathrm{min}$.
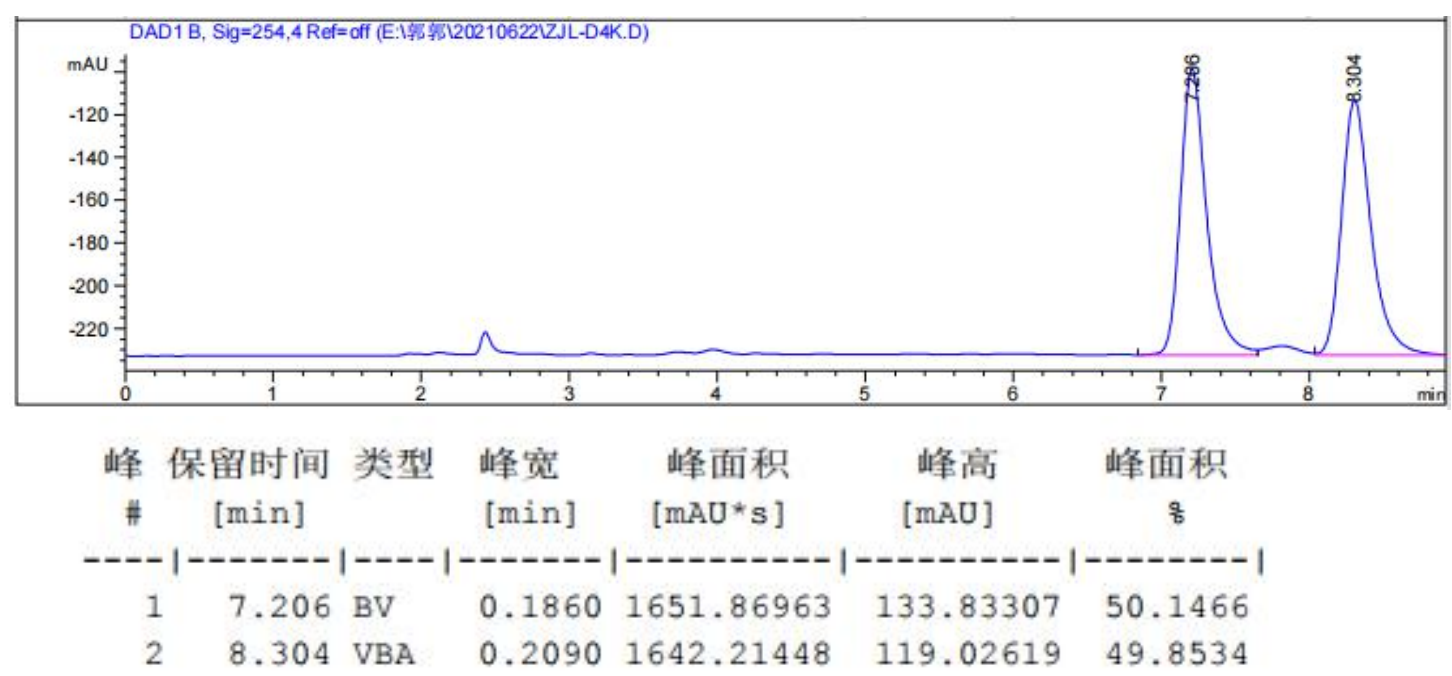